\title{
The Effect of Water Vapor Pressure on the Fatigue Crack Propagation Rates in Aerospace Aluminum Alloys 7075-T651 and 2199-T86
}

\author{
A Thesis \\ Presented to \\ the faculty of the School of Engineering and Applied Science \\ University of Virginia \\ in partial fulfillment \\ of the requirements for the degree \\ Master of Science
}

By

ENS Jennifer L. Jones, USN

May

2015 


\section{Approval Sheet}

The thesis

is submitted in partial fulfillment of the requirements

for the degree of

Master of Science

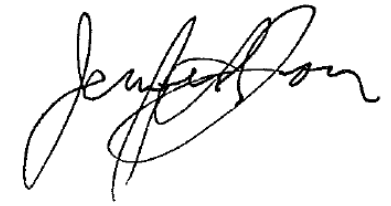

\section{AUTHOR}

This thesis has been read and approved by the examining Committee:

\begin{tabular}{c}
$\frac{\text { Dr. James T. Burns }}{\text { Advisor }}$ \\
Dr. Richard P. Gangloff \\
Committee Chair \\
Dr. Sean R. Agnew \\
\hline
\end{tabular}

Accepted for the School of Engineering and Applied Science:

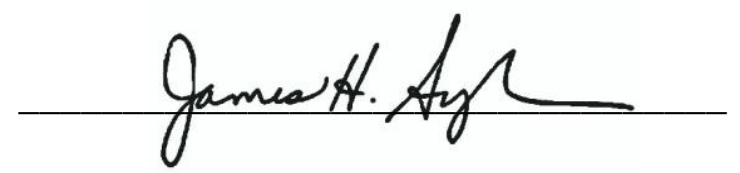

Dean, School of Engineering and Applied Science

May 


\title{
The effect of water vapor pressure on the fatigue crack propagation
}

\author{
rates in aerospace aluminum alloys 7075-T651 and 2199-T86
}

\author{
ENS Jennifer L. Jones, USN
}

\begin{abstract}
The objective of this research is to examine the effect of high purity water vapor on fatigue crack growth rates (da/dN) in aluminum alloys (AA7075-T651 and AA2199-T86).

For decreasing $\Delta \mathrm{K}$ testing (either at a constant stress ratio of $\mathrm{R}=0.5$ or constant a maximum stress intensity of $\mathrm{K}_{\max }=16.5 \mathrm{MPa} \sqrt{\mathrm{m}}$ protocols), growth rates declined with decreasing water vapor pressure over a wide range of $\Delta \mathrm{K}$-values. This behavior demonstrated consistency with current environmental theories where crack growth is limited by molecular flow or H-diffusion in the crack tip process zone. A local minimum in $\mathrm{da} / \mathrm{dN}$, referred to as the threshold transition regime (TTR), was observed for low $\Delta \mathrm{K}$ and low to intermediate ranges of water vapor pressures [1]. An initial decline in Stage I growth proceeded towards the UHV threshold before transitioning to increasing da/dN with further decreases in $\Delta \mathrm{K}$ before merging with growth rates typical of high water vapor pressures at very low $\Delta \mathrm{K}$. This behavior was observed for decreasing $\Delta \mathrm{K}$ at both constant $\mathrm{K}_{\max }$ and constant $\mathrm{R}$ testing conditions. The dip in growth rates corresponded to a transition from flat-transgranular (Stage II) cracking to crystallographic slip band cracking (SBC; Stage I) for decreasing da/dN [1].

It is proposed that the $\mathrm{da} / \mathrm{dN}$ minimum is attributed to roughness-induced changes in the mass transport behavior that alters the $\mathrm{P}_{\mathrm{H} 20}$ at the crack tip. As the $\Delta \mathrm{K}$ decreases in low $\mathrm{P}_{\mathrm{H} 2 \mathrm{O}}$ environments, the cross-slip becomes limited which leads to a higher degree of slip band cracking. This increased crack wake roughness impedes the flow of water vapor molecules from the crack mouth to the crack tip, resulting in a decreased crack tip $\mathrm{P}_{\mathrm{H} 20}$ when compared to the $\mathrm{P}_{\mathrm{H} 20}$ of the bulk environment at the crack mouth. The subsequent rise in $\mathrm{da} / \mathrm{dN}$ with decreasing $\Delta \mathrm{K}$ in the TTR is explained based on an increased supply of water vapor to the crack tip due to turbulentconvective mixing. Specifically, after a certain critical level of roughness is reached in the crack
\end{abstract}


wake, the $\mathrm{P}_{\mathrm{H} 20}$ transport mechanism changes to convective turbulent mixing as a result of crack wake asperity contact. The convective mixing increases the supply of $\mathrm{P}_{\mathrm{H} 20}$ to the crack tip that helps to reignite the hydrogen environment embrittlement process and results in the subsequent rise in $\mathrm{da} / \mathrm{dN}$ rates.

This research addresses four primary objectives to validate and extend the threshold transition regime hypothesis presented in the Burns et al. paper [1]. First, quantitatively investigate the crack front evolution in the threshold transition regime to evaluate the proposed molecular transport based explanation. Second, further investigate the interaction between molecular flow and the degree of roughness using targeted experimental evaluation. Third, quantitatively evaluate the degree of asperity contact in the crack wake, as pertinent to the proposed turbulent mixing hypothesis and the post-minima regime behavior. Fourth, extend to characterize the environmental fatigue crack growth behavior of a $3^{\text {rd }}$ generation Al-Cu-Li alloy (2199-T86) with a different slip character than 7075-T651. In toto, this work focuses on understanding the interacting effects of $\mathrm{P}_{\mathrm{H} 20}$, crack wake roughness, and $\Delta \mathrm{K}$ on crack closure and water vapor transport to the crack tip.

The TTR behavior is an environmental effect; specifically associated with the enhanced transport of water vapor molecules from the bulk environment to the crack tip. This process is dependent on crack wake history and bulk $\mathrm{P}_{\mathrm{H} 2 \mathrm{O}}$. The first objective was investigated by completing programed loading sequences at specific $\Delta \mathrm{K}$ of interest to create marks on the fracture surface in order to track the crack front evolution during the threshold transition region. The results showed an irregular crack front evolution in the TTR which suggests that molecular transport in the through thickness dimension controls the environmental influence, particularly in the TTR regime. The results also showed that changing the specimen thickness, and by extension the through thickness dimensions, influences the environmental crack behavior at intermediate exposures.

The second objective investigated the interaction between molecular flow and the degree of roughness through using a test method that kept constant the bulk $\mathrm{P}_{\mathrm{H} 2 \mathrm{O}}, \Delta \mathrm{K}$, and initial crack length, but allowed for different initial spans of crack wake roughness. Constant $\Delta \mathrm{K}, \mathrm{P}_{\mathrm{H} 20} / f$ experiments served to show that the interaction between the roughness on the fracture surface and the molecular transport can result in an order of magnitude change in growth rates directly following crack growth of increased roughness. Additionally, the fractography suggests an 
environmentally affected irregular crack front driven by the interaction of molecular transport, closure, asperity based mixing, and position dependent $\Delta \mathrm{K}$.

The degree of asperity contact in the crack wake, as pertinent to the proposed turbulent mixing hypothesis and the post-minima regime behavior was investigated through developing a quantitative method to indicate the presence of crack wake asperity contact based on comparing the localized roughness and crack opening displacement was developed. These results suggest that the start of crack wake asperity contact does not correspond directly to an increase in da/dN; counter to the proposed molecular transport hypothesis which stated that enhanced crack growth rates corresponded to the start of crack wake asperity contact occurring. The chosen contact metric indicates the presence of crack wake asperity contact, but the role it plays in controlling the upturn in $\mathrm{da} / \mathrm{dN}$ rates until the Mode II displacement occurring is accurately measured. These results do suggest that the upturn in $\mathrm{da} / \mathrm{dN}$ is driven by the interaction of molecular transport, closure, asperity based mixing, and position dependent $\Delta \mathrm{K}$.

Finally, the environmental fatigue crack growth behavior of a $3^{\text {rd }}$ generation Al-Cu-Li alloy (2199-T86) was characterized using a decreasing $\Delta \mathrm{K}$ at a constant $\mathrm{R}$ loading protocol. Fatigue crack growth rates in 2199-T86 (L-T) were seen to decrease with decreasing water vapor pressure over a wide stress intensity range $(\Delta \mathrm{K})$. Despite different global cracking morphology, the similarities in the scope and span of the roughness transition in 2199-T86 suggest a similar threshold transition regime that depends on $\Delta K$ and the environment similar to the molecular transport based theory developed for 7075-T65.

Analysis detailed above indicates that the threshold transition behavior observed in 7075T651 and 2199-T86 is governed by molecular transport that can be affected by the loading protocol, specimen geometry, and/or testing configuration. While the false threshold behavior resulting in the dip in $\mathrm{da} / \mathrm{dN}$ is real and repeatable, such behavior is geometry and molecular flow path dependent and should not be incorporated into fracture mechanics based predictions. Following appropriate testing procedures would help to ensure data that accurately represent the increased fatigue resistance of 7075-T651 and 2199-T86 at high altitudes. The understanding developed in this investigation will better inform protocols in selecting environment appropriate crack growth rates for linear elastic fracture mechanics (LEFM) modeling. 


\section{Table of Contents}

Abstract. 1

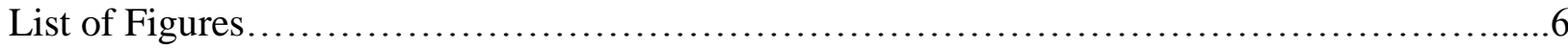

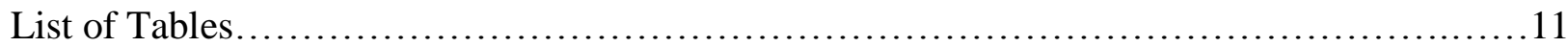

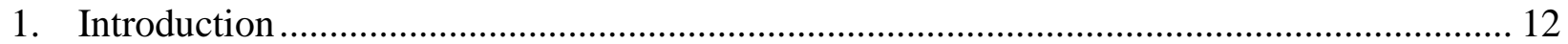

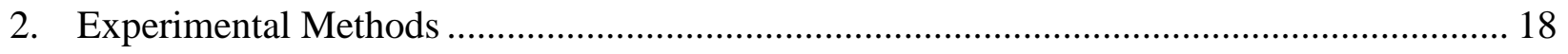

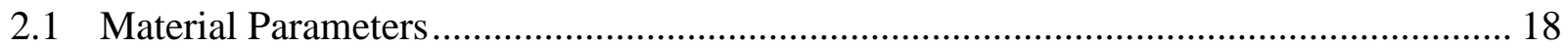

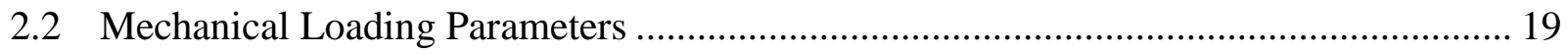

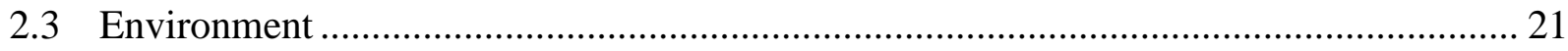

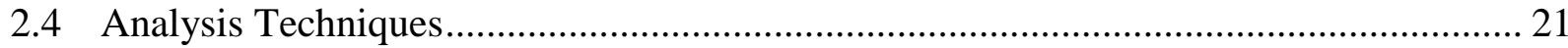

2.4.1 Crack Closure Metrics .................................................................................. 21

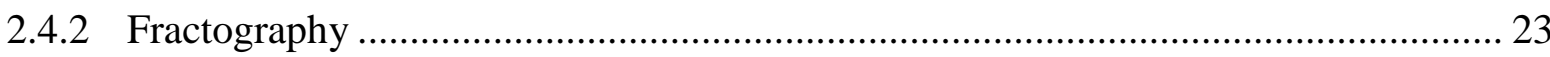

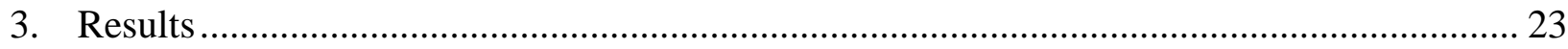

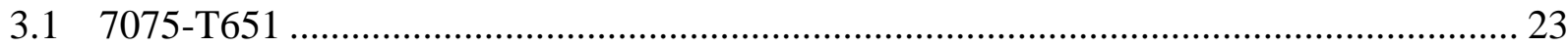

3.1.1 Fatigue Crack Growth Kinetics....................................................................... 23

3.1.2 Fracture Surface Morphology ............................................................................... 24

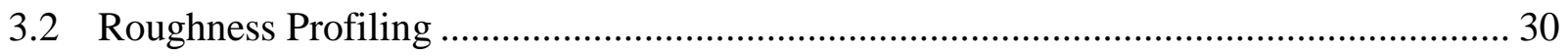

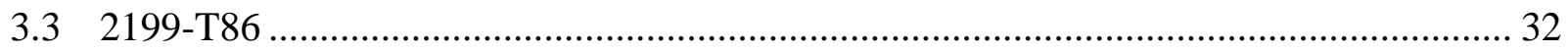

3.3.1 Fatigue Crack Growth Kinetics..................................................................... 32

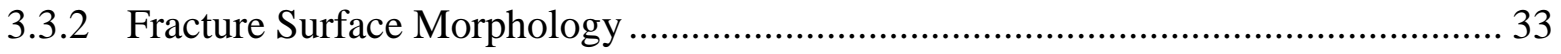

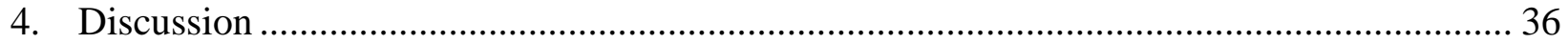

4.1 The Effect of Surface Roughness on Molecular Transport ........................................ 37

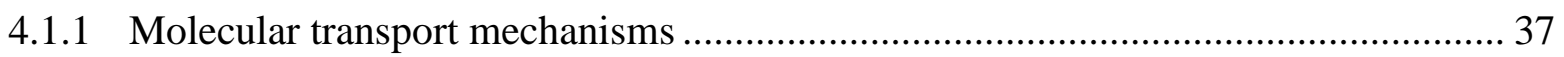

4.1.2 Initial influences of crack wake roughness on $\mathrm{da} / \mathrm{dN}$ behavior $(<5 \mathrm{~mm}$ of crack

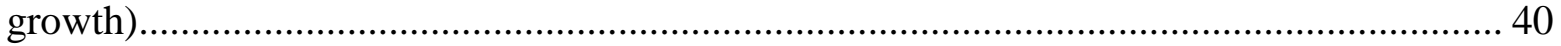

4.2 Crack Front Evolution in the Threshold Transition Regime ...................................... 44

4.3 Post Minima Regime Behavior for 7075-T651 ................................................... 49

4.3.1 Crack Wake Induced Turbulent Mixing ....................................................... 51

4.3.2 Influence of crack wake roughness $10-15 \mathrm{~mm}$ behind the crack tip on molecular

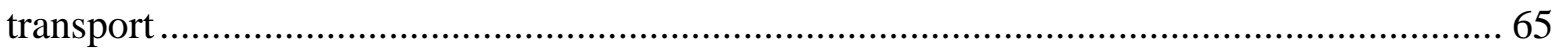

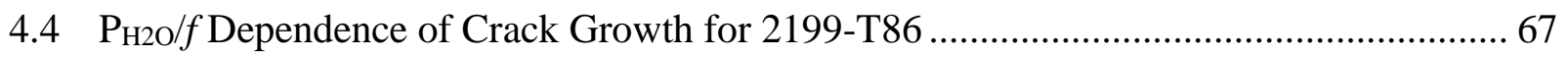

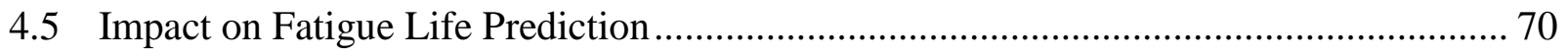

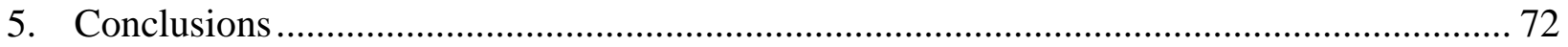




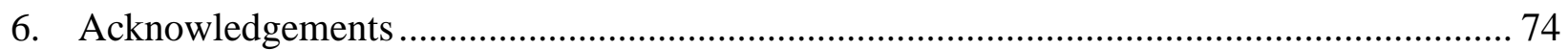

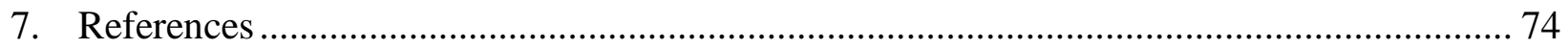

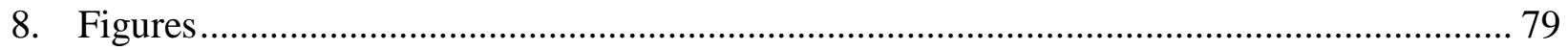

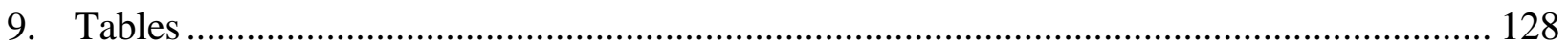




\section{List of Figures}

Figure 1: Fatigue crack growth rate versus decreasing $\Delta \mathrm{K}$ at constant $\mathrm{R}$ of $0.50(\mathrm{f}=20 \mathrm{~Hz})$ for 7075 T651 (L-T) at various-constant water vapor exposure levels from ultra-high vacuum $(0.25-0.50 \mu \mathrm{Pa}-\mathrm{s})$ to $1.3 \mathrm{kPa}-\mathrm{s}\left(\mathrm{P}_{\mathrm{H} 2 \mathrm{O}}=26.7 \mathrm{kPa}\right)[1]$.

Figure 2: Effect of high purity water vapor exposure parameter on fatigue crack growth rate in 7075$\mathrm{T} 651(\mathrm{~L}-\mathrm{T})$ stressed at various-constant $\Delta \mathrm{K}$ levels, each at fixed $\mathrm{R}$ of 0.50 and $\mathrm{f}=20 \mathrm{~Hz}$, taken from the wide-range decreasing $\Delta \mathrm{K}$ data in Figure 1 [1].

Figure 3: Optical fractographs for decreasing $\Delta \mathrm{K}$ at constant $\mathrm{R}$ of $0.50(\mathrm{f}=20 \mathrm{~Hz})$ for $7075-\mathrm{T} 651$ (L-T) at various-constant water vapor exposure levels: (a) Ultra-high vacuum, (b) 0.2, (c) 0.5, (d) 1.8, (e) 4, (f) 18, (g) 38, (h) 165, (i) 340, (j) 2668, and (k) $2668 \mathrm{~Pa} \mathrm{(f=2} \mathrm{Hz).} \mathrm{Crack} \mathrm{growth} \mathrm{is} \mathrm{from} \mathrm{left} \mathrm{to} \mathrm{right} \mathrm{in} \mathrm{each}$ image.

Figure 4: Crack growth rate versus $\Delta \mathrm{K}$ (a) and corresponding optical fractograph (b) illustrating the transition from macroscopically smooth to rough topography on the fatigue crack surface of 7075-T651 (L-T orientation) stressed in pure water vapor at $\mathrm{P}_{\mathrm{H} 2 \mathrm{O}}=0.5 \mathrm{~Pa}$. For constant $\mathrm{R}, \Delta \mathrm{K}$ decreased with increasing crack length from right to left in this image ....

Figure 5: SEM images of the fatigue crack surface of 7075-T651 tested at constant $\mathrm{R}=0.5, \mathrm{f}=20 \mathrm{~Hz}$ (from Figure 1) for an L-T oriented specimen exposed to $\mathrm{P}_{\mathrm{H} 2 \mathrm{O}}$ of: UHV (a-b) and high humidity (c-d) with a $\Delta \mathrm{K}$ of: $(\mathrm{a}, \mathrm{c}) \sim 5,(\mathrm{~b}, \mathrm{~d}) \sim 9 \mathrm{MPa} \sqrt{\mathrm{m}}$. Crack growth is from left to right in each image.

Figure 6: SEM images of the fatigue crack surface of 7075-T651 tested at constant $\mathrm{R}=0.5, \mathrm{f}=20 \mathrm{~Hz}$ and constant $\Delta \mathrm{K}=5 \mathrm{MPa} \sqrt{\mathrm{m}}$ (from Figure 1) for an L-T oriented specimen exposed to $\mathrm{P}_{\mathrm{H} 2 \mathrm{O}}$ of: (a) UHV (b) 0.2, (c) 0.5, (d) 1.8, (e) 4, (f) 18, (g) 38, (h) 165, (i) 340, (j) 2668, and (k) $2668 \mathrm{~Pa} \mathrm{(f} \mathrm{=2} \mathrm{Hz).}$ Crack growth is from left to right in each image.

Figure 7: Higher magnification SEM images of the fatigue crack surface of 7075-T651 tested at constant $\mathrm{R}=0.5, \mathrm{f}=20 \mathrm{~Hz}$ and constant $\Delta \mathrm{K}=5 \mathrm{MPa} \sqrt{\mathrm{m}}$ (from Figure 1 and Figure 8) for an L-T oriented specimen exposed to $\mathrm{P}_{\mathrm{H} 2 \mathrm{O}}$ of: (a) UHV (b) 0.2, (c) 0.5, (d) 1.8, (e) 4, (f) 18, (g) 38, (h) 165, (i) 340, (j) 2668 , and $(\mathrm{k}) 2668 \mathrm{~Pa}(\mathrm{f}=2 \mathrm{~Hz})$. Crack growth is from left to right in each image.

Figure 8: SEM images of the fatigue crack surface of 7075-T651 tested at constant $\mathrm{R}=0.5, f=20 \mathrm{~Hz}$ and constant $\Delta \mathrm{K}=9 \mathrm{MPa} \sqrt{\mathrm{m}}$ (from Figure 1) for an L-T oriented specimen exposed to $\mathrm{P}_{\mathrm{H} 2 \mathrm{O}}$ of: (a) UHV (b) 0.2, (c) 0.5, (d) 1.8, (e) 4, (f) 18, (g) 38, (h) 165, (i) 340, (j) 2668, and (k) $2668 \mathrm{~Pa}(f=2 \mathrm{~Hz}$. Crack growth is from left to right in each image.

Figure 9: SEM images of the fatigue crack surface of 7075-T651 tested at constant $\mathrm{R}=0.5, \mathrm{f}=20 \mathrm{~Hz}$ and constant $\Delta \mathrm{K}=3 \mathrm{MPa} \sqrt{\mathrm{m}}$ (from Figure 1) for an L-T oriented specimen exposed to $\mathrm{P}_{\mathrm{H} 2 \mathrm{O}}$ of: (a) 0.2 , (b) 0.5 , (c) 1.8, (d) 4, (e) 18, (f) 38, (g) 165, (h) 340, (i) 2668, and (j) $2668 \mathrm{~Pa}$ (f =2 Hz). Crack growth is from left to right in each image.

Figure 10: SEM images of the fatigue crack surface of 7075-T651 tested under decreasing $\Delta \mathrm{K}$ loading at constant $\mathrm{R}=0.5, \mathrm{f}=20$ for an $\mathrm{L}-\mathrm{T}$ oriented specimen exposed to $\mathrm{P}_{\mathrm{H} 2 \mathrm{O}}=0.5 \mathrm{~Pa}$ with $\Delta \mathrm{K}$ of: (a) $\sim 7$, (b) $\sim 6$, (c) $\sim 5$, and (d) $\sim 4.0 \mathrm{MPa} \sqrt{ } \mathrm{m}$. The direction of crack growth is left to right in each image.

Figure 11: SEM overview of fracture surface produced from a Kshed loading protocol at a $\mathrm{P}_{\mathrm{H} 2 \mathrm{O}}=0.5 \mathrm{~Pa}$ with vertical compliance calculated crack fronts (blue) and 3 experimental crack fronts identified by marker-bands (green/yellow lines). The green lines correspond to marker-bands identified in the fracture surface and the yellow lines are the inferred crack fronts used to connect the observed marker-bands. Example marker-bands produced at each $\Delta \mathrm{K}$ location are provided below the overview fracture surface for each $\Delta \mathrm{K}$ value. For constant $\mathrm{R}, \Delta \mathrm{K}$ decreased with increasing crack length from left to right in this 
image. The blue lines correspond to the compliance crack lengths associated with a $\Delta \mathrm{K}$ of: (a) 5, (b) 4 , and (c) $3 \mathrm{MPa} \sqrt{\mathrm{m}}$.

Figure 12: Crack growth rate versus $\Delta \mathrm{K}$ (a) and corresponding optical fractograph (b) illustrating the transition from macroscopically rough to smooth topography on the fatigue crack surface of 7075-T651 (L-T orientation) stressed in pure water vapor at $\mathrm{P}_{\mathrm{H} 2 \mathrm{O}}=0.5 \mathrm{~Pa}$. For constant $\mathrm{R}, \Delta \mathrm{K}$ decreased with increasing crack length from right to left in this image. The $0.5 \mathrm{~Pa}$ exposure showed a spike in $\mathrm{da} / \mathrm{dN}$ approximately at $\Delta \mathrm{K}=3.9 \mathrm{MPa} \sqrt{\mathrm{m}}$.

Figure 13: Crack growth rate versus $\Delta \mathrm{K}$ (a) and corresponding optical fractograph (b) illustrating the transition from macroscopically rough to smooth topography on the fatigue crack surface of 7075-T651 (L-T orientation) stressed in pure water vapor at $\mathrm{P}_{\mathrm{H} 2 \mathrm{O}}=0.2 \mathrm{~Pa}$. For constant $\mathrm{R}, \Delta \mathrm{K}$ decreased with increasing crack length from right to left in this image. The $0.2 \mathrm{~Pa}$ exposure showed a spike in da/dN approximately at $\Delta \mathrm{K}=3.6 \mathrm{MPa} \sqrt{\mathrm{m}}$.

Figure 14: (a) Fatigue crack growth rate versus crack length at constant $\Delta K=5 \mathrm{MPa} \sqrt{\mathrm{m}}$ and $\mathrm{R}$ of 0.50 $(\underline{\mathrm{f}}=20 \mathrm{~Hz})$ for $7075-\mathrm{T} 651(\mathrm{~L}-\mathrm{T})$ at $\mathrm{P}_{\mathrm{H} 2 \mathrm{O}}=0.5 \mathrm{~Pa} .0 .5 \mathrm{~mm}$ of crack wake roughness was imparted into the surface before beginning the exposure testing. (b) Optical fractograph of the test specimen corresponding to the crack length scale of the above and below plots. Crack growth occurred left to right. (c) Closure analysis (ASTM 2\% and ACR ratio) versus crack length.

Figure 15: Optical fractograph of the $0.5 \mathrm{~mm}$ of crack wake roughness test specimen tested at $\mathrm{P}_{\mathrm{H} 2 \mathrm{O}}=0.5$ $\mathrm{Pa}$. The SEM images below correspond to the number location where the red dots are on the optical fracture surface. Crack growth occurred left to right.

Figure 16: (a) Fatigue crack growth rate versus crack length at constant $\Delta \mathrm{K}=5 \mathrm{MPa} \sqrt{\mathrm{m}}$ and $\mathrm{R}$ of 0.50 $(\mathrm{f}=20 \mathrm{~Hz})$ for $7075-\mathrm{T} 651(\mathrm{~L}-\mathrm{T})$ at $\mathrm{P}_{\mathrm{H} 2 \mathrm{O}}=0.5 \mathrm{~Pa} .3 \mathrm{~mm}$ of crack wake roughness was imparted into the surface before beginning the exposure testing. (b) Optical fractograph of the test specimen corresponding to the crack length scale of the above and below plots. Crack growth occurred left to right. (c) Closure analysis (ASTM 2\% and ACR ratio) versus crack length...

Figure 17: Optical fractograph of the $3 \mathrm{~mm}$ of crack wake roughness test specimen tested at $\mathrm{P}_{\mathrm{H} 2 \mathrm{O}}=0.5$ $\mathrm{Pa}$. The SEM images below correspond to the number location where the red dots are on the optical fracture surface. Crack growth occurred left to right.

Figure 18: (a) Fatigue crack growth rate versus crack length at constant $\Delta K=3 \mathrm{MPa} \sqrt{\mathrm{m}}$ and $\mathrm{R}$ of 0.50 $(\mathrm{f}=20 \mathrm{~Hz})$ for $7075-\mathrm{T} 651(\mathrm{~L}-\mathrm{T})$ at $\mathrm{P}_{\mathrm{H} 2 \mathrm{O}}=0.5 \mathrm{~Pa}$. No crack rack roughness or load effects were imparted into the surface before beginning the exposure testing. (b) Optical fractograph of the test specimen corresponding to the crack length scale of the above and below plots. Crack growth occurred left to right. (c) Closure analysis (ASTM $2 \%$ and ACR ratio) versus crack length. 101

Figure 19: (a) 3D characterization and the corresponding optical fractograph of the threshold transition regime area for the 7075-T651 1.8 Pa sample produced under decreasing $\Delta \mathrm{K}$ loading at constant $\mathrm{R}$ of $0.50(\mathrm{f}=20 \mathrm{~Hz}$ ), (b) 3D characterization of the $1.8 \mathrm{~Pa}$ sample with a horizontal line scan (shown by the black line). The plot on the right graphs the localized topography of the crack face corresponding to the horizontal line segment. From this plot, the local roughness parameters ( $\mathrm{Ra}$ - average roughness, $\mathrm{Rq}-$ geometric average roughness, $\mathrm{Rp}$ - average peak height, Rv- average valley height) can be gleaned for the horizontal line segment of interest.

Figure 20: (a) 3D characterization and the corresponding optical fractograph of the threshold transition regime area for the 7075-T651 $0.5 \mathrm{~Pa}$ sample produced under decreasing $\Delta \mathrm{K}$ loading at constant $\mathrm{R}$ of $0.50(\mathrm{f}=20 \mathrm{~Hz}),(\mathrm{b}) 3 \mathrm{D}$ characterization of the $0.5 \mathrm{~Pa}$ sample with a horizontal line scan (shown by the black line). The plot on the right graphs the localized topography of the crack face corresponding to the horizontal line segment. From this plot, the local roughness parameters ( $\mathrm{Ra}$ - average roughness, $\mathrm{Rq}-$ 
geometric average roughness, $\mathrm{Rp}$ - average peak height, $\mathrm{Rv}$ - average valley height) can be gleaned for the horizontal line segment of interest. 103

Figure 21: (a) 3D characterization and the corresponding optical fractograph of the threshold transition regime area for the 7075-T651 0.2 Pa sample produced under decreasing $\Delta \mathrm{K}$ loading at constant $\mathrm{R}$ of $0.50(\mathrm{f}=20 \mathrm{~Hz})$, (b) 3D characterization of the $0.2 \mathrm{~Pa}$ sample with a horizontal line scan (shown by the black line). The plot on the right graphs the localized topography of the crack face corresponding to the horizontal line segment. From this plot, the local roughness parameters ( $\mathrm{Ra}$ - average roughness, $\mathrm{Rq}-$ geometric average roughness, $\mathrm{Rp}$ - average peak height, Rv-average valley height) can be gleaned for the horizontal line segment of interest.

Figure 22: $\quad$ Fatigue crack growth rate versus decreasing $\Delta \mathrm{K}$ at constant $\mathrm{R}$ of $0.50(\mathrm{f}=20 \mathrm{~Hz}) \mathrm{for} 2199$ T86 (L-T) at various-constant water vapor exposure levels from ultra-high vacuum $(0.25-0.50 \mu \mathrm{Pa}-\mathrm{s})$ to $1.3 \mathrm{kPa}-\mathrm{s}\left(\mathrm{P}_{\mathrm{H} 2 \mathrm{O}}=26.7 \mathrm{kPa}\right)$. 105

Figure 23: Optical image of the notch for the 2199-T86 un-grooved (left) and side-grooved (right) C-T specimens. Additionally, a small notch was added to the preexisting CT notch through electrical discharge machining (EDM) for the side-grooved specimens. The side-grooving process reduced the overall thickness of the specimen in the crack plane.

Figure 24: Fatigue crack growth rate versus decreasing $\Delta \mathrm{K}$ comparison for the 2199-T86 side-grooved and un-grooved C-T specimens at constant $\mathrm{R}$ of $0.50(\mathrm{f}=20 \mathrm{~Hz})$ for three constant water vapor exposure levels from ultra-high vacuum $(0.25-0.50 \mu \mathrm{Pa}-\mathrm{s})$ to $165 \mathrm{~Pa}$. The growth rate data for the side-grooved specimens are open circles and the un-grooved specimens are labeled as "regular" and are shown as crosses.

Figure 25: Effect of water vapor exposure on the observed crack growth rate minima in the threshold transition regime for 7075-T651 loaded under constant $\mathrm{K}_{\max }(16.5 \mathrm{MPa} \sqrt{\mathrm{m}})$-decreasing $\Delta \mathrm{K}$ in both L-T and T-L orientations as well as constant $\Delta \mathrm{K}$-constant $\mathrm{R}(0.5)$ loading in the $\mathrm{L}$-T orientation (Figure 1 ) as shown in previous work [1]. Additionally, the effect of water vapor exposure on the observed crack growth rate minima in the threshold transition regime for 2199-T86 loaded under decreasing $\Delta \mathrm{K}$ in the LT orientation (Figure 22) is plotted. 108

Figure 26: Optical fractographs for decreasing $\Delta \mathrm{K}$ at constant $\mathrm{R}$ of $0.50(\mathrm{f}=20 \mathrm{~Hz})$ for 2199-T86 (L-T) at various-constant water vapor exposure levels: (a) Ultra-high vacuum, (b) 0.5, (c) 1.8, (d) 4, (e) 18, (f) 38 , and $(\mathrm{g}) 2668 \mathrm{~Pa}$. Crack growth is left to right in each image.

Figure 27: Crack growth rate versus $\Delta \mathrm{K}$ (a) and corresponding optical fractograph (b) illustrating the transition from macroscopically smooth to rough topography on the fatigue crack surface of 2199-T86 (L$\mathrm{T}$ orientation) stressed in pure water vapor at $\mathrm{P}_{\mathrm{H} 2 \mathrm{O}}=1.8 \mathrm{~Pa}$. For constant $\mathrm{R}, \Delta \mathrm{K}$ decreased with increasing crack length from right to left in this image.

Figure 28: $\quad$ SEM images of the fatigue crack surface of 2199-T86 tested at constant $R=0.5, f=20$ (from Figure 22) for an L-T oriented specimen exposed to $\mathrm{P}_{\mathrm{H} 2 \mathrm{O}}$ of: UHV (a-b) and high humidity (2668 $\mathrm{Pa})(\mathrm{c}-\mathrm{d})$ with a $\Delta \mathrm{K}$ of: $(\mathrm{a}, \mathrm{c}) \sim 5,(\mathrm{~b}, \mathrm{~d}) \sim 9 \mathrm{MPa} \sqrt{\mathrm{m}}$. Crack growth is from left to right in each image. 111

Figure 29: SEM images of the fatigue crack surface of 2199-T86 tested at constant $\mathrm{R}=0.5, \mathrm{f}=20 \mathrm{~Hz}$ and constant $\Delta \mathrm{K}=7 \mathrm{MPa} \sqrt{ } \mathrm{m}$ (from Figure 22) for an L-T oriented specimen exposed to $\mathrm{P}_{\mathrm{H} 2 \mathrm{O}}$ of: (a) UHV (b) 0.5, (c) 1.8, (d) 4, (e) 18, (f) 38, and (g) 2668 Pa. Crack growth is from left to right in each image.

Figure 30: Higher magnification SEM images of the fatigue crack surface of 2199-T86 tested at constant $\mathrm{R}=0.5, \mathrm{f}=20 \mathrm{~Hz}$ and constant $\Delta \mathrm{K}=7 \mathrm{MPa} \sqrt{\mathrm{m}}$ (from Figure 22) for an L-T oriented specimen exposed to $\mathrm{P}_{\mathrm{H} 2 \mathrm{O}}$ of: (a) UHV (b) 0.5, (c) 1.8, (d) 4, (e) 18, (f) 38, and (g) $2668 \mathrm{~Pa}$. Crack growth is from left to right in each image. 
Figure 31: SEM images of the fatigue crack surface of 2199-T86 tested at constant $\mathrm{R}=0.5, \mathrm{f}=20 \mathrm{~Hz}$ and constant $\Delta \mathrm{K}=9 \mathrm{MPa} \sqrt{\mathrm{m}}$ (from Figure 22) for an L-T oriented specimen exposed to $\mathrm{P}_{\mathrm{H} 2 \mathrm{O}}$ of: (a) UHV (b) 0.5, (c) 1.8, (d) 4, (e) 18, (f) 38, and (g) $2668 \mathrm{~Pa}$. Crack growth is from left to right in each image.

Figure 32: SEM images of the fatigue crack surface of 2199-T86 tested at constant $\mathrm{R}=0.5, \mathrm{f}=20 \mathrm{~Hz}$ and constant $\Delta \mathrm{K}=5 \mathrm{MPa} \sqrt{\mathrm{m}}$ (from Figure 22) for an L-T oriented specimen exposed to $\mathrm{P}_{\mathrm{H} 2 \mathrm{O}}$ of: (a) UHV (b) 0.5 , (c) 1.8, (d) 4, (e) 18, (f) 38, and (g) $2668 \mathrm{~Pa}$. Crack growth is from left to right in each image.

Figure 33: SEM images of the fatigue crack surface of 2199 - T86 tested under decreasing $\Delta \mathrm{K}$ loading at constant $\mathrm{R}=0.5, \mathrm{f}=20$ for an $\mathrm{L}-\mathrm{T}$ oriented specimen exposed to $\mathrm{P}_{\mathrm{H} 2 \mathrm{O}}=1.8 \mathrm{~Pa}$ with $\Delta \mathrm{K}$ of: (a) $\sim 9$, (b) $\sim 7$, (c) $\sim 6$, and (d) $\sim 5 \mathrm{MPa} \sqrt{\mathrm{m}}$. The direction of crack growth is left to right in each image.

Figure 34: (a) Schematic of crack front progression and corresponding optical fractograph stressed in pure water vapor at $\mathrm{P}_{\mathrm{H} 2 \mathrm{O}}=0.5 \mathrm{~Pa}$ at constant $\Delta \mathrm{K}=5 \mathrm{MPa} \sqrt{\mathrm{m}}$ and $\mathrm{R}$ of $0.50(\mathrm{f}=20 \mathrm{~Hz})$ for 7075-T651 (L-T). $0.5 \mathrm{~mm}$ of crack wake roughness was imparted into the surface before beginning the exposure testing. (b) Schematic of crack front progression and corresponding optical fractograph stressed in pure water vapor at $\mathrm{P}_{\mathrm{H} 2 \mathrm{O}}=0.5 \mathrm{~Pa}$ at constant $\Delta \mathrm{K}=5 \mathrm{MPa} \sqrt{\mathrm{m}}$ and $\mathrm{R}$ of $0.50(\mathrm{f}=20 \mathrm{~Hz})$ for 7075-T651 (L-T). $3 \mathrm{~mm}$ of crack wake roughness was imparted into the surface before beginning the exposure testing. Crack front evolution progresses left to right and each number corresponds to a continuous crack front. The increased roughness portion of the crack front is represented by triangles and the transgranular/flat portion of the crack front is identified by a straight line. The black line in both images outlines the increased roughness portion of the fracture surface.

Figure 35: Schematic of crack front progression and corresponding optical fractograph stressed in pure water vapor at $\mathrm{P}_{\mathrm{H} 2 \mathrm{O}}=0.5 \mathrm{~Pa}$. For constant $\mathrm{R}, \Delta \mathrm{K}$ decreased with increasing crack length from right to left in this image. Crack front evolution progresses left to right and each number corresponds to a continuous crack front. The increased roughness portion of the crack front is represented by triangles and the transgranular/flat portion of the crack front is identified by a straight line. The black line in both images outlines the increased roughness portion of the fracture surface.

Figure 36: (a) C(T) specimen geometry and various locations where crack-line displacements were calculated according to the Newman method [75]. Newman represented the crack face profile from the crack tip to the load line as a straight line. (b) C(T) specimen geometry and various locations where crack-line displacements were calculated according to the Saxena method [76]. The red lines represent the crack face profile for a crack length of $\mathrm{a}_{0}$ which is used to represent the elastic behavior of crack growth near the crack tip. $V_{0}$ corresponds to the crack mouth opening for both diagrams. (c) Crack profile schematic of the combined model and $\mathrm{C}(\mathrm{T})$ specimen geometry that calculates crack mouth opening displacement (CMOD) using elastic theory and linear modeling of the crack surface: $\mathrm{x}$ is a specific crack length location of interest along the crack wake, $\mathrm{a}_{0}$ is the current crack length factoring in the axis of rotation described previously, and $\theta$ is the angle of displacement between the crack surface and the crack tip opening displacement (CTOD).

Figure 37: Schematic of a C(T) specimen [53] and a C(T) fatigue crack growing with a portion of increased roughness in the crack wake. As the crack continues to grow (left to right in the diagram above), the COD calculations still reflect the crack-opening displacement at the location of roughness indicated by the red line.

Figure 38: (a) Crack asperity schematic illustrating a condition where the Mode II displacement is equal to the Mode I displacement. This condition would represent the minimum or lower bound of Mode II displacement that would cause asperity contact. (b) Crack asperity schematic illustrating a condition where the amount of Mode II displacement results in $80 \%$ of the crack asperity height remaining in contact with the opposing face during loading. This situation was quantified as having a magnitude of 
$20 \%$ of the total crack asperity height $(0.2 * \mathrm{Rpv})$. (c) Crack asperity schematic illustrating a condition where the Mode II displacement results in opposing crack asperities that are directly aligned, resulting in peaks and valleys directly opening and closing on top of each other. This condition would represent the maximum or upper bound of Mode II displacement that would cause asperity contact. The magnitude of the total crack asperity height (Rpv) was used to quantify this displacement condition with the localized roughness.

Figure 39: (a) Crack-opening displacement (COD) calculations at the location corresponding to the start of increased roughness $(\mathrm{a}=27.5 \mathrm{~mm})$ for the $1.8 \mathrm{~Pa}$ exposure specimen plotted as a function of crack length under maximum and minimum loading. Additionally, the respective height of the crack asperities under two Mode II displacement conditions ( $0.2 \mathrm{Rpv}$ and $0.0069 \mathrm{Rpv})$ are plotted. (b) Optical fractograph of the test specimen corresponding to the crack length scale of the above and below plots with the black arrow indicating the location where the COD was calculated. Crack growth occurred left to right. (c) Closure analysis (ASTM 2\% and ACR ratio) versus crack length.

Figure 40: The effective crack opening $\left(\delta_{\text {eff }}\right)$ over the nominal crack opening $\left(\delta_{\text {nom }}=\right.$ COD at the maximum load - COD at the minimum load) is plotted as a function of crack length for two Mode II displacement conditions ( $0.2 \mathrm{Rpv}$ and $0.0069 \mathrm{Rpv}$ ) and two locations ( $\mathrm{a}=27.5$ and $30.0 \mathrm{~mm}$ ) for the $1.8 \mathrm{~Pa}$ specimen. The optical fractograph of the test specimen, corresponding to the crack length scale of the above plot, indicates the two locations where the COD was calculated. 122

Figure 41: (a) Crack-opening displacement (COD) calculations at the location corresponding to the start of increased roughness $(\mathrm{a}=21.9 \mathrm{~mm})$ for the $0.5 \mathrm{~Pa}$ exposure specimen plotted as a function of crack length under maximum and minimum loading. Additionally, the respective height of the crack asperities under two Mode II displacement conditions (0.2 Rpv and 0.0037 Rpv) are plotted. (b) Optical fractograph of the test specimen corresponding to the crack length scale of the above and below plots with the black arrow indicating the location where the COD was calculated. Crack growth occurred left to right. (c) Closure analysis (ASTM 2\% and ACR ratio) versus crack length.

Figure 42: The effective crack opening $\left(\delta_{\text {eff }}\right)$ over the nominal crack opening $\left(\delta_{\text {nom }}=\right.$ COD at the maximum load - COD at the minimum load) is plotted as a function of crack length for two Mode II displacement conditions ( $0.2 \mathrm{Rpv}$ and $0.0037 \mathrm{Rpv})$ and two locations $(\mathrm{a}=27.5$ and $30.0 \mathrm{~mm})$ for the $0.5 \mathrm{~Pa}$ specimen. The optical fractograph of the test specimen, corresponding to the crack length scale of the above plot, indicates the two locations where the COD was calculated.

Figure 43: (a) Crack-opening displacement (COD) calculations at the location corresponding to the start of increased roughness $(\mathrm{a}=29.6 \mathrm{~mm})$ for the $0.2 \mathrm{~Pa}$ exposure specimen plotted as a function of crack length under maximum and minimum loading. Additionally, the respective height of the crack asperities under two Mode II displacement conditions ( $0.2 \mathrm{Rpv}$ and $0.0069 \mathrm{Rpv})$ are plotted. (b) Optical fractograph of the test specimen corresponding to the crack length scale of the above and below plots with the black arrow indicating the location where the COD was calculated. Crack growth occurred left to right. (c) Closure analysis (ASTM 2\% and ACR ratio) versus crack length.

Figure 44: The effective crack opening $\left(\delta_{\text {eff }}\right)$ over the nominal crack opening $\left(\delta_{\text {nom }}=\right.$ COD at the maximum load - COD at the minimum load) is plotted as a function of crack length for two Mode II displacement conditions ( $0.2 \mathrm{Rpv}$ and $0.0025 \mathrm{Rpv}$ ) and two locations ( $\mathrm{a}=29.6$ and $34.7 \mathrm{~mm}$ ) for the $0.2 \mathrm{~Pa}$ specimen. The optical fractograph of the test specimen, corresponding to the crack length scale of the above plot, indicates the two locations where the COD was calculated.

Figure 45: LEFM modeling (AFGROW) formatted fatigue crack growth rate versus decreasing $\triangle \mathrm{K}$ at constant $\mathrm{R}$ of $0.50(\mathrm{f}=20 \mathrm{~Hz})$ for 7075-T651 $(\mathrm{L}-\mathrm{T})$ at various-constant water vapor exposure levels from ultra-high vacuum $(0.25-0.50 \mu \mathrm{Pa}-\mathrm{s})$ to $1.3 \mathrm{kPa}-\mathrm{s}\left(\mathrm{P}_{\mathrm{H} 2 \mathrm{O}}=26.7 \mathrm{kPa}\right)$. The plotted data represents 30 data point sets (Table 8) that are taken from the results from Figure 1. The threshold transition regime has been removed for the $1.8,0.5$, and $0.2 \mathrm{~Pa}$ exposures. 


\section{List of Tables}

Table 1: Weighted average $\mathrm{Ra}$ and $\mathrm{Rq}$ values taken in the threshold transition regime for 0.2 , 0.5 , and $1.8 \mathrm{~Pa}$. 128

Table 2: Weighted average $\mathrm{Ra}$ and $\mathrm{Rq}$ values taken after the threshold transition regime for 0.2 , 0.5 , and $1.8 \mathrm{~Pa}$. 128

Table 3: Weighted average $\mathrm{Ra}$ and $\mathrm{Rq}$ values taken for 4 and $2668 \mathrm{~Pa}$ 128

Table 4: Largest $\mathrm{Rp}$ and $\mathrm{Rv}$ values observed in the threshold transition regime for $0.2,0.5$, and 1.8 Pa. Rpv is the sum of Rp and Rv and reflects the total magnitude of the crack face roughness.

Table 5: The magnitude of the fracture surface roughness span for the $0.2,0.5$, and $1.8 \mathrm{~Pa}$ exposures.

Table 6: The CTOD, Mode II displacement, and the corresponding percentage of Rpv for the condition where Mode II/Mode I $=0.3$ for the $0.2,0.5$, and $1.8 \mathrm{~Pa}$ exposures. These values were calculated at crack lengths corresponding to the start of the roughness span.

Table 7: The 3 Mode II displacement conditions and the corresponding magnitude of the crack asperities for the $0.2,0.5$, and $1.8 \mathrm{~Pa}$ exposures.

Table 8: AFGROW formatted fatigue crack growth rate versus decreasing $\Delta \mathrm{K}$ at constant $\mathrm{R}$ of $0.50(\mathrm{f}=20 \mathrm{~Hz})$ for 7075-T651 (L-T) at various-constant water vapor exposure levels from ultrahigh vacuum $(0.25-0.50 \mu \mathrm{Pa}-\mathrm{s})$ to $1.3 \mathrm{kPa}-\mathrm{s}\left(\mathrm{P}_{\mathrm{H} 2 \mathrm{O}}=26.7 \mathrm{kPa}\right.$. The data (plotted in Figure 42$)$ represents 30 data point sets that are taken from the results in Figure 1. The threshold transition regime has been removed for the $1.8,0.5$, and $0.2 \mathrm{~Pa}$ exposures. 


\section{Introduction}

Crack progression under fatigue loading is governed by cyclic plastic damage accumulation that can be described by the stress intensity range $\left(\Delta \mathrm{K}=\mathrm{K}_{\max }-\mathrm{K}_{\min }\right)$ or the maximum stress intensity $\left(\mathrm{K}_{\max }\right)$ [1-5]. The stress intensity factor, $\mathrm{K}$, can be used independent of the geometry of the sample to describe the crack tip driving force [77]. From the principle of similitude, fatigue cracks grow at an equal rate $(\mathrm{da} / \mathrm{dN})$ when subjected to equal $\Delta \mathrm{K}$, implying that the stress intensity factor range uniquely defines the crack-tip conditions [77]. Laboratory crack growth rate data are often coupled with linear elastic fracture mechanics (LEFM)-based approaches to predict the crack extension in engineering components using an assumed initial flaw size and loading representative of the service conditions. Essentially, fatigue crack progression (FCP) life prediction integrates the $\mathrm{da} / \mathrm{dN}$ associated with a given crack size and stress (thus $\Delta \mathrm{K}$ ) in order to predict the amount of crack extension. LEFM prognosis models have made substantial progress in modeling crack growth, but remain limited in regards to incorporating metallurgical factors, transient loading conditions, and the environment [6-10].

Airframe structures experience a substantial amount of fatigue loading during operation. Depending on the aircraft type, a significant proportion of this loading takes place at high altitude where the external environment is at low temperatures and low water pressures. Inflight loading for airframe components occurs in environments ranging from ambient temperature and high humidity (low altitudes) to low-temperature and low water vapor pressure (high altitudes) [9]. Specifically, recent work has focused on characterizing the operational environment of military and transport aircraft to help better inform and guide laboratory testing of aluminum aerospace alloys [11]. Similar work has also been performed to obtain a relevant coupled loadingenvironment spectrum (ENSTAFF) to assist in the prognosis of composite structures [12]. 
Researchers found that a majority of fatigue loading in fighter aircraft wings occurred during aggressive maneuvering performed at high altitudes $(\sim 30,000 \mathrm{ft})[11,13-14]$. Takeoffs, landings, and wind gusts provide the highest amount of fatigue loading on the wings for transport vehicles $[13,15-17]$. De Jonge et al. found that $17 \%$ and $42 \%$ of transport aircraft wing loads exceeding 0.3G's occur above 30,000 and 10,000 feet, respectively, suggesting a significant amount of loading is taking place in environments where low temperatures and low water vapor pressures are prevalent $[13,17]$.

For precipitation hardened aluminum 7xxx-series alloys, moist gaseous environments have been shown to significantly impact fatigue crack propagation $(\mathrm{FCP})$ rates $(\mathrm{da} / \mathrm{dN})$ under varying loading conditions [18-22]. Crack growth is strongly influenced by chemical and electrochemical considerations within the crack [23]. Both temperature and water vapor pressure impact fatigue crack formation and propagation $[1,13]$. Specifically, decreasing the temperature and/or water vapor pressure results in an orders-of-magnitude decrease in fatigue crack growth rates for the aerospace aluminum alloys 7075-T651 and 2199-T86 [1, 13]. The dependence of da/dN on the effective water vapor pressure $\left(\mathrm{P}_{\mathrm{H} 20} / f\right)$ has been quantified and modeled based on the hydrogen environment embrittlement (HEE) perspective on crack growth kinetics put forth by Wei and coworkers. Specifically, the crack growth kinetics are affected by the slowest step in a sequence of events involving water-molecular transport from crack mouth to crack tip, crack surface reactions, and the diffusion of atomic hydrogen into the microstructure $[10,18]$. Researchers propose that at high altitudes, moisture will condense to form an ice layer on the surface of a component resulting in a local water vapor pressure at the surface that is given by the gas equilibrium above solid ice at a given temperature $\left(\mathrm{P}_{\mathrm{H} 20 \text {-ice }}\right)[13,24]$. While prior work has shown that the water vapor pressure over frequency $\left(\mathrm{P}_{\mathrm{H} 2 \mathrm{O}} / f\right)$ exposure parameter does not fully capture 
the effect of temperature, it can be used as a conservative proxy to model the environmental influence on fatigue cracking [25 - 26]. Efforts are underway to investigate the beneficial effect of low temperature on the fatigue crack growth behavior beyond that associated with the reduction in the $\mathrm{P}_{\mathrm{H} 2 \mathrm{O}}$ [25]. However, this work will focus on establishing and understanding the effect of varying $\mathrm{P}_{\mathrm{H} 2 \mathrm{O}}$ at room temperature $(23 \mathrm{C})$ on the cracking kinetics.

Research has established that for high strength aerospace $\mathrm{Al}$ alloys, da/dN is dependent on the interaction of $\Delta \mathrm{K}, \mathrm{K}_{\max }$, and $\mathrm{P}_{\mathrm{H} 20} / f$ [1]. An extensive amount of work has been completed to understand and incorporate variable amplitude loading effects into lifetime modeling of fatigue damage $[1,13,27]$. However, there remains a need to increase understanding of the strong influence of the environment on fatigue cracking $[13,27]$. Specifically, there remains a deficiency in incorporating the effect of a coupled load-environment spectrum (specifically, variable environments and variable amplitude loading) into LEFM based modeling [1]. Incorporating the effect of high altitude environments on da/dN rates in LEFM models would increase accuracy and reduce over-conservatism in current real world applications. Current fracture mechanic-based prognosis methods often use ambient temperature-moist air fatigue properties to predict cracking in these high altitude environments, which can lead to highly conservative life predictions [28-29]. It is necessary to quantify and understand the effect of these loading environments on the crack growth kinetics in order to reduce this over-conservatism by incorporating such environmental effects into LEFM modeling approaches.

In that pursuit, work performed by Burns and coworkers examined the mechanistic understanding of fatigue crack propagation growth rate response to wide-ranging stress intensity protocol interactions with water vapor exposure in the legacy aluminum alloy 7075-T651 [1]. The authors set out to validate that the parameter, $\mathrm{P}_{\mathrm{H} 2 \mathrm{O}} / f$, accurately captured the relationship between 
loading duration and the hydrogen embrittlement process (HEE) kinetics. Secondly, they focused on exploring the effects of mechanical loading on environmental fatigue through looking at Rratio effects on environment induced crack closure and the $\Delta \mathrm{K}$-dependent environmental influence for various $\mathrm{P}_{\mathrm{H} 20}$ at $23^{\circ} \mathrm{C}[1,13]$.

Fatigue crack growth rates in 7075-T651 (L-T) were shown to decrease with decreasing water vapor pressure over a wide stress intensity range $(\Delta \mathrm{K})$ (Figure 1) [1]. For $\mathrm{P}_{\mathrm{H} 2 \mathrm{O}}$ above $4 \mathrm{~Pa}, \mathrm{da} / \mathrm{dN}$ decreased with decreasing $\Delta \mathrm{K}$ in four power law segments that agreed with governing steps in the hydrogen environment embrittlement (HEE) process and literature [1, 19-21]. Most basically, the hydrogen environmental contribution to $\mathrm{da} / \mathrm{dN}$ is governed by the concentration of atomic hydrogen produced on the crack tip surfaces and subsequent diffusion into the crack tip process zone $[1,18,19]$. Current crack growth kinetics models for Al alloys suggest that this process can be limited by: (1) water vapor molecule transport from the crack mouth to crack tip as governed by Knudsen diffusion, (2) the rate of the $\mathrm{Al}-\mathrm{H}_{2} \mathrm{O}$ surface reaction to produce atomic $\mathrm{H}$, (3) $\mathrm{H}$ diffusion into the crack tip fracture process zone, and (4) H-plasticity stress interaction [30-39]. In the low water vapor exposures, fatigue crack growth is governed purely by crack tip plasticity due to limited hydrogen uptake. At intermediate exposures, da/dN is proportional to the exposure parameter based on the water vapor transport via the Knudsen flow model $[1,25]$. The da/dN dependence on $\mathrm{P}_{\mathrm{H} 2 \mathrm{O}} / f$ and $\Delta \mathrm{K}$ in Figure 1 indicates that the HEE process and crack growth kinetics are sensitive to lowering the water vapor pressure [1].

For decreasing $\Delta \mathrm{K}$ testing (both at constant stress ratio $(\mathrm{R}=0.5)$ and constant maximum stress intensity $\left(\mathrm{K}_{\max }=16.5 \mathrm{MPa} \sqrt{\mathrm{m}}\right)$ protocols $)$, a novel minimum in $\mathrm{da} / \mathrm{dN}$, referred to as the threshold transition regime (TTR), was observed for low $\Delta \mathrm{K}$ and low to intermediate ranges of water vapor pressures [1]. An initial decline in Stage I growth proceeded towards the UHV threshold before 
transitioning to increasing $\mathrm{da} / \mathrm{dN}$ with further decreases in $\Delta \mathrm{K}$ before merging with growth rates typical of high water vapor pressures at very low $\Delta \mathrm{K}[1]$. This behavior was observed for decreasing $\Delta \mathrm{K}$ at both constant $\mathrm{K}_{\max }$ and constant $\mathrm{R}$ testing conditions. The dip in growth rates corresponded to a transition from flat-transgranular (Stage II) cracking to crystallographic slip band cracking (SBC; Stage I) for decreasing da/dN [1]. The authors proposed that the da/dN minimum is attributed to roughness-induced changes in the mass transport behavior that alters the $\mathrm{P}_{\mathrm{H} 20}$ at the crack tip. As the $\Delta \mathrm{K}$ decreases in low $\mathrm{P}_{\mathrm{H} 2 \mathrm{O}}$ environments, the cross-slip becomes limited which leads to a higher proportion of SBC in those low $\mathrm{P}_{\mathrm{H} 2 \mathrm{O}}$ cases where $\mathrm{H}$ production is limited. This increased crack wake roughness impedes the flow of water vapor molecules from the crack mouth to the crack tip, resulting in a decreased crack tip $\mathrm{P}_{\mathrm{H} 20}$ when compared to the $\mathrm{P}_{\mathrm{H} 20}$ of the bulk environment at the crack mouth. Optical and scanning electron microscopy (SEM) images were used to show that the density of faceted features in the TTR increased with decreasing $\mathrm{P}_{\mathrm{H} 2 \mathrm{O}}$, but a more quantitative comparison is needed. The decreased $\mathrm{P}_{\mathrm{H} 20}$ at the crack tip results in higher amounts of slip band cracking which self-perpetuates the steep decrease in $\mathrm{da} / \mathrm{dN}$. Variations in the $\mathrm{da} / \mathrm{dN}$ minima behavior occurred for different testing protocols (constant $\mathrm{K}_{\max }$-decreasing $\Delta \mathrm{K}$, constant R-decreasing $\Delta \mathrm{K}$ ); highlighting the importance of the R-dependent crack wake opening displacement on the mass transport of water vapor to the crack tip [1]. After the initial onset of SBC, $\mathrm{H}$ diffusion and subsequent cracking inwardly from the crack faces could become a contributing factor to the threshold transition region due to the thickness dimension being the pertinent distance for molecular transport to the crack tip. As such, the effect of the pertinent diffusion distance on the crack front progression within the threshold transition regime requires further investigation. 
The subsequent rise in da/dN with decreasing $\Delta \mathrm{K}$ in the TTR is explained based on an increased supply of water vapor to the crack tip due to turbulent-convective mixing. Specifically, after a certain critical level of roughness is reached in the crack wake, the $\mathrm{P}_{\mathrm{H} 20}$ transport mechanism changes to convective turbulent mixing as a result of crack wake asperity contact. The convective mixing increases the supply of $\mathrm{P}_{\mathrm{H} 20}$ to the crack tip that helps to reignite the hydrogen environment embrittlement process and results in the subsequent rise in da/dN rates. Further research is needed to quantitatively evaluate the hypothesis that increasing roughness spans will result in the molecular flow transitioning from being impeded to enhanced. Additionally, better understanding is needed about the controlling mechanisms in the post-minima regime of the TTR; specifically addressing why there is a transition back to transgranular fracture with decreasing $\Delta \mathrm{K}$ and why a second minima is not observed.

Due to the critical importance of the crack wake morphology in the threshold transition regime, it is of interest to investigate the relevancy of this mechanism for a $3^{\text {rd }}$ generation Al-Cu-Li alloy (2199-T86). Al-Cu-Li alloys have shown a strong dependence on moist environments and exhibit a superior fatigue performance when compared to $\mathrm{Al}-\mathrm{Zn}-\mathrm{Mg}-\mathrm{Cu}$ alloys for a wide range of environmental exposures [40-43]. These initial trends could be linked to the shearable $\delta$ '-phase $\left(\mathrm{Al}_{3} \mathrm{Li}\right)$ in $2199-\mathrm{T} 86$ that enables homogenous reversible planar slip [40-44]. Slip band cracking is able to occur at a wide range of $\Delta \mathrm{K}$, which could result in a different environmental dependence at low $\Delta \mathrm{K}$ and low water vapor pressure as seen for 7075-T651. Such differences may alter the molecular flow path that was hypothesized to be critical to the environmental cracking behavior, specifically in the threshold transition regime.

This research will address four primary objectives to validate and extend the threshold transition regime hypothesis presented in the Burns et al. paper [1]. First, quantitatively investigate 
the crack front evolution in the threshold transition regime to evaluate the proposed molecular transport based explanation. Second, further investigate the interaction between molecular flow and the degree of roughness using targeted experimental evaluation. Third, quantitatively evaluate the degree of asperity contact in the crack wake, as it is pertinent to the proposed turbulent mixing hypothesis and the post-minima regime behavior. Fourth, extend to characterize the environmental fatigue crack growth behavior of a $3^{\text {rd }}$ generation Al-Cu-Li alloy (2199-T86) with a different slip character than 7075-T651. This work will help to further understanding of the interacting effects of $\mathrm{P}_{\mathrm{H} 20}$, crack wake roughness, and $\Delta \mathrm{K}$ on crack closure and water vapor transport to the crack tip.

\section{Experimental Methods}

\subsection{Material Parameters}

The fatigue performance of two aluminum aerospace alloys (7075-T651 and 2199-T86) was investigated. A $50.8 \mathrm{~mm}$ thick plate of 7075-T651 was used for experimentation. The 7075T651 plate had a grain size in the rolling-longitudinal (L) direction from 20 to $100 \mu \mathrm{m}, 30$ to 300 $\mu \mathrm{m}$ in the width (transverse, $\mathrm{T}$ ) direction, and 10 to $70 \mu \mathrm{m}$ in the thickness (S) direction [1]. The tensile yield and ultimate strengths in the rolling direction were 508 and $598 \mathrm{MPa}$, respectively [1]. 7075-T651 has a plane strain fracture toughness of $33 \mathrm{MPa} \sqrt{\mathrm{m}}$ [1]. For 2199-T86, the tensile yield and ultimate strengths in the rolling direction were 423 and $461 \mathrm{MPa}$, respectively. 2199T86 has a plane strain fracture toughness of $59.5 \mathrm{MPa} \sqrt{ } \mathrm{m}$ in the L-T orientation and $47 \mathrm{MPa} \sqrt{\mathrm{m}}$ in the T-L orientation [81]. The grain sizes and texture of the 2199-T86 plate were undocumented.

Fatigue crack growth experiments for 7075-T651 were performed on compact tension $(\mathrm{C}(\mathrm{T}))$ specimens machined in the L-T orientation centered at $8.5 \mathrm{~mm}$ from the plate surface (T/7.3) [1]. The specimens had a width and thickness of 50.8 and $7.62 \mathrm{~mm}$ respectively, with a notch depth $12.7 \mathrm{~mm}$ ahead of the load line. The fatigue crack growth rate tests were guided by 
ASTM E647 with the crack length being calculated using unloading compliance from a clip gauge measured crack mouth opening displacement [45]. Testing was performed using two different loading formats to characterize the effect of $\mathrm{P}_{\mathrm{H} 2 \mathrm{O}} / f$ on $\mathrm{da} / \mathrm{dN}$. Both testing protocols were run at a frequency $(f)$ of $20 \mathrm{~Hz}$ and a constant stress ratio $\left(\mathrm{R}=\mathrm{K}_{\min } / \mathrm{K}_{\max }\right)$ of 0.5 .

\subsection{Mechanical Loading Parameters}

The first loading protocol measured growth rates under a decreasing $\Delta \mathrm{K}$ protocol in accordance with $\Delta \mathrm{K}=\Delta \mathrm{K}_{\mathrm{o}} \exp \left[\mathrm{C}\left(\mathrm{a}-\mathrm{a}_{\mathrm{o}}\right)\right]$ where $\Delta \mathrm{K}_{\mathrm{o}}=14.85 \mathrm{MPa} \sqrt{\mathrm{m}}, \mathrm{a}_{0}=12.7 \mathrm{~mm}$, and $\mathrm{C}=0.08$ $\mathrm{mm}^{-1}[1,45]$. Loading decreased from $10 \mathrm{MPa} \sqrt{\mathrm{m}}$ to a threshold $\mathrm{da} / \mathrm{dN}$ value equal to approximately $5 \times 10^{-8} \mathrm{~mm} /$ cycle or when $\Delta \mathrm{K}$ reached $2 \mathrm{MPa} \sqrt{\mathrm{m}}$. Next, an increasing $\Delta \mathrm{K}(\mathrm{C}$ value of $0.2 \mathrm{~mm}^{-1}$ ) segment was run from a $\Delta \mathrm{K}$ of approximately $4 \mathrm{MPa} \sqrt{\mathrm{m}}$ to $12 \mathrm{MPa} \sqrt{\mathrm{m}}$. Growth rates were calculated at a specific $\Delta \mathrm{K}$ using a 7-point incremental polynomial curve fit [45]. The second loading format measured growth rates at low-constant $\Delta \mathrm{K}(3.5,4.5$, and 5.5 $\mathrm{MPa} \sqrt{\mathrm{m}})$. A single $\mathrm{C}(\mathrm{T})$ specimen was used for each $\Delta \mathrm{K}$ level and da/dN rates were measured for varying $\mathrm{P}_{\mathrm{H} 2 \mathrm{O}} / f$ exposures ranging from ultra-high vacuum (UHV) to relative high humidity ( 2668 $\mathrm{Pa}$ ). Each exposure was held constant for approximately $1 \mathrm{~mm}$ of crack growth and a linear regression of crack length versus cycles was utilized to calculate the steady state growth rate.

A third loading protocol was designed to examine the effect of crack wake roughness on $\mathrm{da} / \mathrm{dN}$. Two 7075-T651 samples were tested using a constant $\Delta \mathrm{K}=5 \mathrm{MPa} \sqrt{\mathrm{m}}(\mathrm{R}=0.5, f=20 \mathrm{~Hz})$ testing protocol at a $\mathrm{P}_{\mathrm{H} 20}=0.5 \mathrm{~Pa}$. Before starting the constant $\Delta \mathrm{K}$-testing segment, samples were fatigue cracked at $\mathrm{UHV}(\Delta \mathrm{K}=4.5 \mathrm{MPa} \sqrt{\mathrm{m}}, \mathrm{R}=0.5, f=20 \mathrm{~Hz})$ for specific amounts of crack length $(0.5 \mathrm{~mm}$ and $3 \mathrm{~mm})$ to produce $\mathrm{SBC}$. These spans of increased crack wake roughness were determined based on examining the fracture surface of the $0.5 \mathrm{~Pa}$ exposure under a $\mathrm{K}$-shed loading protocol. The 0.5 Pa fracture surface, produced from the K-shed loading protocol listed above, 
had a roughness span of SBC covering the entire width of the fracture surface that was approximately $0.75 \mathrm{~mm}$ long. The magnitude of crack wake roughness for experimentation was selected to target the da/dN behavior with a roughness span smaller and larger than this critical roughness span $(0.75 \mathrm{~mm})$ as determined from the $\mathrm{K}$-shed testing. The constant $\Delta \mathrm{K}$-testing segment started at the same crack length $(\mathrm{a}=18 \mathrm{~mm})$ for each specimen to ensure similar crack opening displacements throughout both experiments. A fourth loading protocol was performed on a 7075-T651 sample using a constant $\Delta \mathrm{K}=3 \mathrm{MPa} \sqrt{\mathrm{m}}_{\text {(constant }} \mathrm{K}_{\max }, \mathrm{R}=0.5, f=20 \mathrm{~Hz}$ ) testing protocol at $\mathrm{P}_{\mathrm{H} 20}=0.5 \mathrm{~Pa}$. Unlike the previous tests, this sample started constant $\Delta \mathrm{K}$ loading at 0.5 Pa directly after completing the pre-crack at a crack length $\mathrm{a}=13.7 \mathrm{~mm}$. This test was performed to investigate the effect of no loading history, and by extension no crack wake roughness, on da/dN at a $\Delta \mathrm{K}$ lower than the threshold transition regime.

The fifth loading protocol measured growth rates under a decreasing $\Delta \mathrm{K}$ protocol $(\mathrm{C}$-value of $0.08 \mathrm{~mm}^{-1}$ ) from $10 \mathrm{MPa} \sqrt{\mathrm{m}}$ to a threshold $\mathrm{da} / \mathrm{dN}$ value similar to the first loading protocol. During K-shed loading, three marker-bands were inputted into the fracture surface at $\Delta \mathrm{K}$ of 5,4 , and $3 \mathrm{MPa} \sqrt{\mathrm{m}}$ by maintaining a constant $\operatorname{Kmax}$ value $(10,8$, or $6 \mathrm{MPa} \sqrt{\mathrm{m}}$ respectively), but alternating between $\mathrm{R}=0.1, f=10 \mathrm{~Hz}$ and the baseline $\mathrm{R}=0.5, f=20 \mathrm{~Hz}$. For the $\Delta \mathrm{K}=5 \mathrm{MPa} \sqrt{\mathrm{m}}$ loading sequence, a three time repeating sequence (50 marker cycles +5000 baseline cycles) was completed to create a total marker-band series 10 microns in width. At $\Delta \mathrm{K}=4$ and $3 \mathrm{MPa} \sqrt{\mathrm{m}}$, a three time repeating sequence (50 marker cycles +200 baseline cycles) for $\Delta \mathrm{K}=4 \mathrm{MPa} \sqrt{\mathrm{m}}$ and (50 marker cycles +2000 baseline cycles) for $\Delta \mathrm{K}=3 \mathrm{MPa} \sqrt{\mathrm{m}}$ was completed. The loading sequence produced a change in the fracture surface morphology that enabled the crack front at each location of interest to be identified. By inputting markers into the surface, this loading protocol was utilized to target crack front progression during the threshold transition regime. 


\subsection{Environment}

Loading protocols 1 and 2 were performed at 2668, 340, 165, 38, 18, 4, 1.8, 0.5, 0.2 and 5 x $10^{-7}$ (UHV) Pa; corresponding with the equilibrium water vapor pressures above water or ice at roughly $23^{\circ} \mathrm{C}$ (relative humidity of $95 \%$ ), $-4^{\circ} \mathrm{C},-15^{\circ} \mathrm{C},-30^{\circ} \mathrm{C},-37^{\circ} \mathrm{C},-50^{\circ} \mathrm{C},-57^{\circ} \mathrm{C},-65^{\circ} \mathrm{C},-$ $73^{\circ} \mathrm{C}$, and $-90^{\circ} \mathrm{C}$, respectively, according to the Clausius-Clapeyron equation [25]. Loading protocols 3-5 were performed only at $0.5 \mathrm{~Pa}\left(-65^{\circ} \mathrm{C}\right)$. Experiments were completed in a $\mathrm{Cu}-$ gasket-sealed stainless steel UHV chamber where varying levels of pure water vapor were introduced through a sealed glass flask via a leak valve [1]. The total pressure in the chamber was maintained at the desired level through balancing the water vapor input and the pumping of the vacuum system.

\subsection{Analysis Techniques}

\subsubsection{Crack Closure Metrics}

For each loading protocol and testing condition, the effective stress-intensity factor range $\left(\Delta \mathrm{K}_{\text {eff }}\right)$ was calculated using the compliance-based methods outlined in ASTM E647-08 to quantify the degree of crack closure occurring during testing [45]. Crack closure is the phenomenon where fracture surfaces come into contact during the unloading portion of a loading cycle, transferring force across the crack [46]. Crack closure results in a situation where the applied $\Delta \mathrm{K}\left(\Delta \mathrm{K}_{\text {nom }}\right)$ is reduced, which results in an effective stress intensity factor $\left(\Delta \mathrm{K}_{\mathrm{eff}}\right)$. The closure ratio reported in this work for ASTM $2 \%$ and adjusted compliance ratio (ACR) both reflect

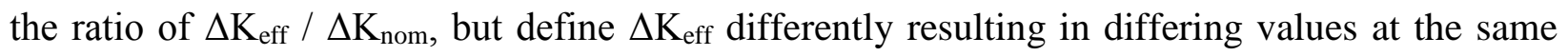
crack length. Both methods will be presented in this study, though it is debated as to which method presents a more rigorous and accurate representation of the presence of crack closure. The difference between the ASTM 2\% and ACR closure correction methods (outlined in Appendix X2 
and X4 of ASTM E647-08) is that the ASTM 2\% method assumes that the stress range below the opening stress does not affect crack growth behavior, whereas the ACR assumes it does [45-48]. The effective stress intensity factor in the ASTM $2 \%$ ratio is calculated by using a least-square fit to the graph of the applied load vs. the corresponding crack mouth opening displacement. The compliance value is determined by the slope of this line and is subsequently used to calculate the compliance offset. The opening force is determined based on a $2 \%$ offset criterion and used to calculate the effective force range that can be directly related to the effective stress intensity factor range $\left(\Delta \mathrm{K}_{\mathrm{eff}}\right)[45]$. The ACR is determined by the equation below:

$$
\mathrm{ACR}=\left(\mathrm{C}_{\mathrm{s}}-\mathrm{C}_{\mathrm{i}}\right) /\left(\mathrm{C}_{\mathrm{o}}-\mathrm{C}_{\mathrm{i}}\right)
$$

where $\mathrm{C}_{\mathrm{i}}$ is the inverse slope of the applied load-crack mouth opening displacement plot prior to initiation, $\mathrm{C}_{\mathrm{s}}$ is the secant compliance, and $\mathrm{C}_{\mathrm{o}}$ is the compliance above the opening load. This ratio considers the bulk shielding mechanism in the wake of the crack and the effect on the cyclic strain field in front of the crack [46]. The effective stress intensity factor range can then be calculated by the equation:

$$
\Delta \mathrm{K}_{\mathrm{eff}}=\mathrm{ACR} * \Delta \mathrm{K}_{\mathrm{nom}}
$$

The ACR method will generally indicate less closure when compared to the ASTM $2 \%$ due to the reduced sensitivity to local crack tip contact. The adjusted compliance ratio and ASTM $2 \%$ indicate no crack closure effects occurring when the ratio is equal to $\Delta \mathrm{K}_{\text {eff }} / \Delta \mathrm{K}_{\text {nom }}=1$. As $\Delta \mathrm{K}_{\text {eff }}$ $/ \Delta \mathrm{K}_{\text {nom }}$ decreases, this represents an increasing effect of crack closure that is reducing the overall effective stress intensity that is being felt at the crack tip. This work normalized the calculated $\Delta \mathrm{K}_{\mathrm{eff}}$ by the nominal $\Delta \mathrm{K}$ as a means to qualitatively compare the degree of crack closure occurring during different testing conditions. 


\subsubsection{Fractography}

Each fracture surface produced by K-shed loading was examined under low magnification optical microscopy. Additionally, SEM (Scanning Electron Microscope) fractography was performed for all exposures looking at key $\Delta \mathrm{K}$ values before, during, and after the threshold transition region. These locations were identified based on the compliance indicated crack front location at the $\triangle \mathrm{K}$ of interest. All SEM images taken were secondary electron images using a 10 $\mathrm{kV}$ accelerating voltage with a working distance ranging from 10 to $20 \mathrm{~mm}$. The fracture surfaces of the $0.2,0.5,1.8,4$, and $2668 \mathrm{~Pa}$ exposures were examined using the Zygo White-Light 3D Surface Profilometer to enable 3-D characterization of the fracture surface and corresponding surface roughness. A 3-D characterization of the fracture surface for the threshold transition region (approximately $8 \mathrm{~mm} \times 10 \mathrm{~mm}$ ) was examined for each sample that exhibited a dip in growth rates $(0.2,0.5,1.8 \mathrm{~Pa})$. The roughness scans of the 4 and $2668 \mathrm{~Pa}$ fracture surfaces were used for baseline comparisons. Each sample was leveled and filtered using a Robust Gaussian filter.

\section{Results}

\section{$3.1 \quad 7075-$ T651}

\subsubsection{Fatigue Crack Growth Kinetics}

Fatigue crack growth rates in 7075-T651 (L-T) were shown to decrease with decreasing water vapor pressure over a wide stress intensity range $(\Delta \mathrm{K})$ and presented in depth in a previous paper [1]. Figure 1 presents the dependence of $\mathrm{da} / \mathrm{dN}$ on $\Delta \mathrm{K}$ and $\mathrm{P}_{\mathrm{H} 2 \mathrm{O}}$ for the L-T orientation of 7075-T651 measured under decreasing $\Delta \mathrm{K}$ at a constant $\mathrm{R}$ of 0.5 and a frequency of $20 \mathrm{~Hz}$ [1]. For $\mathrm{P}_{\mathrm{H} 2 \mathrm{O}}$ above $4 \mathrm{~Pa}, \mathrm{da} / \mathrm{dN}$ decreased with decreasing $\Delta \mathrm{K}$ in four power law segments that are consistent with rate governing steps in the hydrogen environment embrittlement (HEE) process and literature $[1,19-21]$. A novel da/dN versus $\Delta \mathrm{K}$ trend was observed for $\mathrm{P}_{\mathrm{H} 2 \mathrm{O}}$ values of $1.8,0.5$, 
and $0.2 \mathrm{~Pa}$, where da/dN decreased rapidly between a $\Delta \mathrm{K}$ of $6-5 \mathrm{MPa} \sqrt{\mathrm{m}}$. The da/dN minima occurred for a $\Delta \mathrm{K}$ range of 4.7 to $5.0 \mathrm{MPa} \sqrt{\mathrm{m}}$, before systematically increasing with decreasing $\Delta \mathrm{K}$. This region is referred to as the threshold transition regime [1].

The effect of water vapor exposure on $\mathrm{da} / \mathrm{dN}$ for constant $\Delta \mathrm{K}(\mathrm{R}=0.5)$ (Figure 2) shows strong dependence on both $\mathrm{P}_{\mathrm{H} 2 \mathrm{O}}$ and $\Delta \mathrm{K}$ with the following considerations: (1) fatigue crack propagation rates at $\mathrm{UHV}$ and low-rising $\mathrm{P}_{\mathrm{H} 2 \mathrm{O}} / f$ below $10^{-4} \mathrm{~Pa}-\mathrm{s}$, which are environment independent, (2) molecular flow limited da/dN, directly proportional to $\mathrm{P}_{\mathrm{H} 2 \mathrm{O}} / f$ up to about $10^{-2} \mathrm{~Pa}-$ s, and (3) a reduced dependence on exposure perhaps including possibility of an exposure independent $\mathrm{da} / \mathrm{dN}$ plateau controlled by the saturation of the surface reaction occurring on the crack surface $[1,10,21,30]$.

\subsubsection{Fracture Surface Morphology}

Each fracture surface produced by K-shed loading was examined under low magnification optical microscopy and presented in Figure 3 with the decreasing $\Delta \mathrm{K}$ portion of the fracture surface indicated by the red box. For all decreasing $\Delta \mathrm{K}$ experiments between 1.8 and $0.2 \mathrm{~Pa}$ (Figure $3 \mathrm{~b}$ 3d), the specimen's fracture surface showed a transition from a smooth fracture surface to a rough surface in the middle. The roughened region transitioned back to a similar smooth crack surface with increasing crack length and decreasing $\Delta \mathrm{K}$. The change in morphology correlates with the threshold transition region and is shown for the fracture surface of a L-T specimen stressed at $\mathrm{P}_{\mathrm{H} 2 \mathrm{O}}$ $=0.5 \mathrm{~Pa}$ in Figure 4. Lines of matching style between the optical fractograph and the da/dN plot correspond to the compliance indicated crack front location at the $\Delta \mathrm{K}$ of interest; post-test optical crack length corrections varied less than 5\% from the final compliance measured crack length for all of the completed tests. The decrease in the da/dN rates correlates to the increased roughness 
portion of the fracture surface. For testing completed at a $\mathrm{P}_{\mathrm{H} 20}=4 \mathrm{~Pa}$ or higher, the fracture surface had a nearly uniform macroscopic fracture pattern for the entirety of the K-shed loading segment (Figure 3e-3k). The roughness transition seen for the exposures between $1.8 \mathrm{~Pa}$ and UHV suggests a mechanism transition that depends on $\Delta \mathrm{K}$ and the environment [1].

Previous work on precipitation hardened Al alloys showed that the rough faceted features present in inert environments at low $\Delta \mathrm{K}$ (Figure 5a) were characteristic of slip band cracking (SBC) parallel to the $\{111\}$ slip planes [1, 49-50]. Slip band cracking was not present at higher $\Delta \mathrm{K}$ (Figure $5 \mathrm{~b}, 5 \mathrm{~d}$ ) for UHV and high humidity, which has been attributed to the activation of multiple slip systems as the stress intensity range and crack tip plasticity increases [1]. A noncrystallographic transgranular morphology that also included flat void-like features, suggestive of fatigue cracking around constituent particles, was observed in the fracture surface at higher $\Delta \mathrm{K}$ and high humidity (Figure 5d) [1, 51].

The fracture surface morphology varied with water vapor pressure ranging from UHV to relative high humidity (2668 Pa) (Figure 6) when compared at a $\Delta \mathrm{K}=5 \mathrm{MPa} \vee \mathrm{m}$. The fraction of faceted features systematically decreased with increasing water vapor pressure. SBC-like features were first observed at $\mathrm{P}_{\mathrm{H} 2 \mathrm{O}}=1.8 \mathrm{~Pa}$ (Figure $6 \mathrm{~d}$ and $7 \mathrm{~d}$ ) with increasing occurrence as $\mathrm{P}_{\mathrm{H} 2 \mathrm{O}}$ decreased to UHV. At higher exposures (>1.8 Pa, Figures 6e-6k and 7e-7k), the crack morphology is fully non-crystallographic transgranular, which is typical of $\mathrm{H}$-assisted cracking. Additionally, the fracture surface morphology was compared before and after the threshold transition regime at $\Delta \mathrm{K}=9$ and $3 \mathrm{MPa} \sqrt{\mathrm{m}}$, respectively. The fracture surface morphologies before the threshold transition regime showed transgranular fracture patterns for all tested exposures at $\Delta \mathrm{K}=9 \mathrm{MPa} \sqrt{\mathrm{m}}$ (Figure 8). The fracture surface morphologies after the threshold transition regime at $\Delta \mathrm{K}=3$ $\mathrm{MPa} \sqrt{ } \mathrm{m}$ showed transgranular fracture patterns for exposures greater than $0.2 \mathrm{~Pa}$ (Figure 9). For 
the UHV exposure, the stress intensity range was below crack growth threshold, so no image was available for comparison. In summary, prominent variations in the fracture surfaces between the exposures were only seen at an intermediate $\Delta \mathrm{K}(5 \mathrm{MPa} \sqrt{\mathrm{m}})$ and low water vapor pressures (Figures 6b-6d and 7b-7d).

As shown in prior work [1], the fracture surface morphology has been used to interpret the threshold transition behavior as an occurrence related to a limitation in the governing mechanisms of the HEE process. The fracture surface morphology in the threshold transition regime showed a transition from transgranular fracture to SBC-like morphology that was consistent with the lowmagnification optical images (Figures 3 and 4) [1]. Figure 10 shows SEM images of the fracture surface for the $0.5 \mathrm{~Pa}$ exposure at specific $\Delta \mathrm{K}$ across the threshold transition regime. Flat transgranular fracture patterns were seen at $\Delta \mathrm{K}$ values of 7 and $6 \mathrm{MPa} \sqrt{\mathrm{m}}$ (Figure $9 \mathrm{a}$ and $9 \mathrm{~b}$ ), which corresponded to growth rates slightly before the onset of the dip in da/dN. SBC-like features were present at a $\Delta \mathrm{K}$ value of $5 \mathrm{MPa} \sqrt{\mathrm{m}}$ that corresponded to the minimum of the dip in $\mathrm{da} / \mathrm{dN}$ (Figure 10c). As the $\Delta \mathrm{K}$ decreased to $4 \mathrm{MPa} \sqrt{\mathrm{m}}$ (Figure 10d), the crack surface morphology showed decreasing amounts of SBC-like features before transitioning to fully flat transgranular fracture and higher growth rates for low $\Delta \mathrm{K}$. The more severe dips in growth rate corresponded to lower $\mathrm{P}_{\mathrm{H} 2 \mathrm{O}}$ values and a higher fraction of SBC-like features. Since the degree of SBC increases with decreasing HEE, the microscopic feature changes during the threshold transition regime are indicative of a minimum amount of HEE occurring at the da/dN minima [1].

\subsubsection{Crack Front Evolution in the Threshold Transition Regime}

The crack front evolution during the threshold transition region was examined by completing programed loading sequences at $\Delta \mathrm{K}$ of 5,4 , and $3 \mathrm{MPa} \sqrt{\mathrm{m}}$ to create marks on the 
fracture surface. Figure 11 is an overview image of the complete fracture surface with the crack fronts (blue lines) calculated from the compliance and the experimental crack front found from marker-banding (green/yellow lines) for each of the three $\Delta \mathrm{K}$ values. The green lines correspond to marker-bands identified in the fracture surface and the yellow lines are the inferred crack fronts used to connect the observed marker-bands and identify the continuous crack front. Example marker-bands produced at each $\Delta \mathrm{K}$ location are provided below the overview fracture surface for each $\Delta \mathrm{K}$ value. The calculated and experimental crack fronts for $\Delta \mathrm{K}=5 \mathrm{MPa} \sqrt{\mathrm{m}}$ are similar in location and shape suggesting strong correlation between the compliance calculated crack front and the experimental data at the start of the threshold transition region. The experimental crack fronts for $\Delta \mathrm{K}=4$ and $3 \mathrm{MPa} \sqrt{\mathrm{m}}$ are more elliptical in nature suggesting faster cracking on the sides then in the middle of the sample. The crack front found from the $\Delta \mathrm{K}=4 \mathrm{MPa} \sqrt{\mathrm{m}}$ markerbands has a steeper radius of curvature than at a $\Delta \mathrm{K}=3 \mathrm{MPa} \sqrt{\mathrm{m}}_{\text {. }}$

The irregular crack shape seen at $\Delta \mathrm{K}=4$ and $3 \mathrm{MPa} \sqrt{ }$ m could lead to inaccurate compliance and stress intensity solutions during the threshold transition regime. Figure 12 plots the dependence of $\mathrm{da} / \mathrm{dN}$ on $\Delta \mathrm{K}$ and $\mathrm{P}_{\mathrm{H} 2 \mathrm{O}}$ for the L-T orientation of 7075-T651 measured under decreasing $\Delta \mathrm{K}(\mathrm{R}=0.5, f=20 \mathrm{~Hz})$; specifically highlighting the low $\Delta \mathrm{K}$ behavior of the $0.5 \mathrm{~Pa}$ exposure. A rapid increase or spike in $\mathrm{da} / \mathrm{dN}$ on the order of two magnitudes occurred for a $\Delta \mathrm{K}$ range of 3.7 to $4.0 \mathrm{MPa} \sqrt{\mathrm{m}}$. After the spike, growth rates returned to systematically decreasing $\mathrm{da} / \mathrm{dN}$ with decreasing $\Delta \mathrm{K}$. Figure 12 correlates the location of the spike in growth rates to the optical fractograph of the $0.5 \mathrm{~Pa}$ exposure. Lines of matching style between the optical fractograph and the $\mathrm{da} / \mathrm{dN}$ plot correspond to the compliance indicated crack front location at the $\Delta \mathrm{K}$ of interest. The rapid spike in the $\mathrm{da} / \mathrm{dN}$ rates correlates to the vertical span of the crack face with approximately $0.75 \mathrm{~mm}$ of SBC in the center of the specimen surrounded by transgranular fracture 
from both crack flanks. Growth rates after the spike correlate to a fracture surface that is fully transgranular. The subsequent spike in $\mathrm{da} / \mathrm{dN}$ after the TTR was also observed for the $0.2 \mathrm{~Pa}$ exposure and plotted in Figure 13. The spike in da/dN for the $0.2 \mathrm{~Pa}$ sample had a similar magnitude as the $0.5 \mathrm{~Pa}$ sample, but occurred for a $\Delta \mathrm{K}$ range of 3.3 to $3.7 \mathrm{MPa} \sqrt{\mathrm{m}}$. Unlike the 0.5 Pa sample, the growth rates after the spike did not systematically decrease, but instead fluctuated with decreasing $\Delta \mathrm{K}$. The difference between the compliance calculated and experimental crack fronts at these post da/dN minima $\Delta \mathrm{K}$ values will be addressed in the Discussion as well as the potential impact of inaccurate compliance and stress intensity solutions on da/dN during the TTR.

\subsubsection{Impeded/Enhanced Molecular Flow Experimentation}

Two 7075-T651 samples with different magnitudes of crack wake roughness were tested using a constant $\Delta \mathrm{K}=5 \mathrm{MPa} \sqrt{\mathrm{m}}(\mathrm{R}=0.5, f=20 \mathrm{~Hz})$ loading protocol at a $\mathrm{P}_{\mathrm{H} 20}=0.5 \mathrm{~Pa}$ to study the effect of roughness on $\mathrm{da} / \mathrm{dN}$. The selection process for the different magnitudes of crack wake roughness $(0.5$ and $3 \mathrm{~mm})$ was described previously in the Experimental methods. Figures 14 and 16 plot the da/dN behavior vs crack length for each specimen with the corresponding fracture surface underneath (scaled to match the crack lengths in the plot above). Both experiments show that a steady state da/dN growth rate was not achieved in the long term, but instead showed oscillating trends with growing crack length. The growth rate for $\Delta \mathrm{K}=5 \mathrm{MPa} \sqrt{\mathrm{m}}$, estimated from the K-shed experiment (Figure 1), is da/dN $=4.0 \times 10^{-6} \mathrm{~mm} /$ cycle and was used as the comparison to determine whether the roughness experiments were experiencing impeded or enhanced growth rates. For the $0.5 \mathrm{~mm}$ sample, da/dN rates were impeded and slowly increased for approximately $2 \mathrm{~mm}$ before beginning to oscillate around a steady state growth rate approximately $3.0 \times 10^{-6}$ $\mathrm{mm} /$ cycle. For the $3 \mathrm{~mm}$ sample, da/dN rates were impeded for approximately $1 \mathrm{~mm}$ before 
becoming enhanced for $1 \mathrm{~mm}$. After the first two millimeters of crack growth, growth rates began to oscillate around a steady state growth rate approximately $2.0 \times 10^{-6} \mathrm{~mm} / \mathrm{cycle}$ in a more symmetric pattern than seen in the $0.5 \mathrm{~mm}$ sample. Both samples showed an increased amount of SBC corresponding to subsequent decreases in the da/dN shown in Figures 15 and 17.

Figures 14 and 16 also plot the closure ratios associated with the ASTM 2\% and ACR methods vs crack length for each specimen scaled to match the crack lengths in the fracture surface and $\mathrm{da} / \mathrm{dN}$ plot above. The ACR results for the $0.5 \mathrm{~mm}$ sample (Figure $14 \mathrm{c}$ ) show that crack closure is accounting for approximately a 5 to $15 \%$ reduction in the stress intensity factor for the entire duration of the test, whereas the ASTM $2 \%$ only indicates the presence of crack closure for a crack length range from 26 to $30 \mathrm{~mm}$. The ACR results for the $3 \mathrm{~mm}$ sample (Figure 16c) show that crack closure is accounting for approximately a 5 to $13 \%$ reduction in the stress intensity factor for the entire duration of the test, whereas the ASTM $2 \%$ indicates a $20 \%$ reduction in the stress intensity factor only for the first four millimeters of crack growth. The fluctuations in $\mathrm{da} / \mathrm{dN}$ and the corresponding fracture surfaces will be addressed in the Discussion.

A third 7075-T651 sample with no prior loading history, and by extension no crack wake

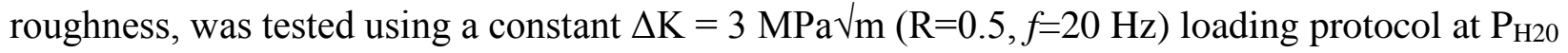
$=0.5 \mathrm{~Pa}$ to study the da/dN behavior at a $\Delta \mathrm{K}$ lower than the threshold transition regime. A plot of the $\mathrm{da} / \mathrm{dN}$ behavior vs crack length for the specimen with the corresponding fracture surface underneath (scaled to match the crack lengths in the plot above) showed a near steady state, but mildly increasing da/dN approximately equal to $1.0 \times 10^{-6} \mathrm{~mm} /$ cycle over $18 \mathrm{~mm}$ of crack growth (Figure 18a). This steady state growth rate equaled the growth rate, da/dN $=1.0 \times 10^{-6} \mathrm{~mm} / \mathrm{cycle}$, estimated from the $\mathrm{K}$-shed experiment at a $\Delta \mathrm{K}=3 \mathrm{MPa} \sqrt{\mathrm{m}}$ (Figure 1). The fractography of the surface revealed transgranular fracture for the duration of the test (Figure 18b). A plot of the 
ASTM $2 \%$ and ACR closure ratios vs crack length (Figure 18c) only showed a less than $3 \%$ reduction in the applied $\Delta \mathrm{K}$ suggesting minimal effects of closure for the duration of the test.

\subsection{Roughness Profiling}

Key 7075-T61 fracture surfaces were examined using the Zygo White-Light 3D Surface Profilometer to enable 3-D characterization of the fracture surface and corresponding surface roughness pertaining to the TTR. Figures 19a, 20a, and 21a show the 3-D characterization of the fracture surface and the corresponding optical fractograph for the 1.8, 0.5, and $0.2 \mathrm{~Pa}$ samples, respectively. Figures 19b, 20b, and 21b highlight example horizontal line scans, oriented with respect to the $\mathrm{C}(\mathrm{T})$ center line in the notch direction, which correlate to the plot of the localized topography of the corresponding crack face. The distance on the $\mathrm{x}$-axis of the plot corresponds with the length of the horizontal line segment and the y-axis is the localized roughness for the selected segment width. Amplitude parameters ( $\mathrm{Ra}, \mathrm{Rq}, \mathrm{Rp}$, and $\mathrm{Rv}$ ) are provided for each line segment and corresponding crack face topography plot. For each sample, the crack length of highest roughness was determined by identifying the highest arithmetic average roughness (Ra). The Ra parameter is the arithmetic average height of the peaks and valleys as determined from a mean line within the sampling length [52]. The geometric average roughness (Rq) was also used as a comparison tool during characterization. Rq is the geometric average height of the roughnesscomponents from a mean line within a sampling length. The $\mathrm{Rq}$ parameter is more sensitive to extremely high or low peak/valley values resulting in a reading that will be approximately $11 \%$ higher than the Ra value for a given surface [52]. For this study, the $\mathrm{Ra}$ and $\mathrm{Rq}$ parameters were used as lower and upper bounds respectively for the roughness of features in the fracture surface at a given crack length. 
The average $\mathrm{Ra}$ and $\mathrm{Rq}$ values for the three dip exposures $(0.2,0.5$, and $1.8 \mathrm{~Pa})$ and two higher humidity exposures (4 and $2668 \mathrm{~Pa}$ ) are presented in Tables 1-3. In order to more accurately represent the average $\mathrm{Ra}$ and $\mathrm{Rq}$ values for the entire width of the crack face, the total horizontal width $(7.56 \mathrm{~mm})$ was broken into approximately $0.5-1 \mathrm{~mm}$ subsections each with corresponding amplitude parameters. The amplitude parameters from the small subsections were used in a weighted average to calculate the total average $\mathrm{Ra}$ and $\mathrm{Rq}$ for the total segment (Tables 1-3). Table 1 shows that crack face topography roughness increased with decreasing $\mathrm{P}_{\mathrm{H} 2 \mathrm{O}}$. After the threshold transition regime, the surface roughness of the $0.2,0.5$, and $1.8 \mathrm{~Pa}$ exposures decreased to values similar to the average roughness measured for the 4 and $2668 \mathrm{~Pa}$ exposures (Tables 2 and 3).

The local crack face topography was examined to determine the average height of crack asperities. The roughness parameters $\mathrm{Rp}$ and $\mathrm{Rv}$ (average height of the peak/valley from a mean line within a sampling) were taken at the location of highest roughness and listed in Table 4 . The $\mathrm{Rp}$ and Rv values were added together to represent the average total height of a crack wake asperity; listed as "Rpv" (Table 4). The average Rp and Rv values increased with decreasing $\mathrm{P}_{\mathrm{H} 2 \mathrm{O}}$, which agreed well with the previous roughness measurements above (Table 1). Additionally, the span of the localized roughness was measured for each the three TTR exposures and presented in Table 5. The magnitude of the roughness span increased with decreasing $\mathrm{P}_{\mathrm{H} 2 \mathrm{O}}$. The depth and roughness profile information is used in conjunction with crack-opening displacement (COD) calculations and ASTM 2\%/ACR closure ratio analysis to evaluate the proposed roughness induced molecular transport theories in the Discussion. 


\section{$3.3 \quad 2199-$ T86}

\subsubsection{Fatigue Crack Growth Kinetics}

Fatigue crack growth rates, as a function of $\Delta \mathrm{K}$ and $\mathrm{P}_{\mathrm{H} 2 \mathrm{O}}$, are shown for 2199-T86 in Figure 22; plot scales are similar to those used in Figure 1 to enable direct comparison of the TTR trends between 7075-T651 and 2199-T86. Figure 22 presents the dependence of da/dN on $\Delta \mathrm{K}$ and $\mathrm{P}_{\mathrm{H} 2 \mathrm{O}}$ for the L-T orientation of 2199-T86 measured under decreasing $\Delta \mathrm{K}$ at a constant $\mathrm{R}$ of 0.5 and a frequency of $20 \mathrm{~Hz}$.

Initial 2199-T86 specimens demonstrated out of plane cracking, associated with inherent anisotropy and the high constraint (thus out of plane T-stress) in CT specimens, for low and intermediate water vapor exposures [53]. As such, a side-grooving protocol was performed on e C(T) specimens consistent with ASTM standard E399 [53]. In addition, a small notch was added to the preexisting $\mathrm{C}(\mathrm{T})$ notch through electrical discharge machining (EDM) to mitigate crack formation along the notched flanks (Figure 23). Verification testing of the 2199-T86 side-grooved specimens was conducted at $38 \mathrm{~Pa}$ and UHV to investigate the procedure's effect on the crack growth rate date between exposure levels (Figure 24). The side-grooved and non-side-grooved specimens showed identical crack growth rate behavior at UHV suggesting that the crack mechanics and $\Delta \mathrm{K}$ solutions are accurate for the new specimens. However, side-grooved specimens tested at intermediate exposures (open circles in Figure 24) showed faster crack growth rates than the non-grooved specimens (crosses in Figure 24). The da/dN rates at $38 \mathrm{~Pa}$ for the sidegrooved specimen showed similar rates to the non-grooved specimen data tested at $165 \mathrm{~Pa}$. The data presented in Figure 20 were completed with all side-grooved specimens except for the two tests completed at $2668 \mathrm{~Pa}$. 
Fatigue crack growth rates in 2199-T86 (L-T) were seen to decrease with decreasing water vapor pressure over a wide stress intensity range $(\Delta \mathrm{K})$ (Figure 22$)$. For $\mathrm{P}_{\mathrm{H} 2 \mathrm{O}}$ above $38 \mathrm{~Pa}, \mathrm{da} / \mathrm{dN}$ decreased with decreasing $\Delta \mathrm{K}$ in four power law segments consistent with the HEE process [1, 19-21]. The equivalence of the da/dN rates observed at 0.5 Pa and UHV in 2199-T86 illustrates the elimination of environmental influences at a higher exposure than 7075-T651. A rapid decrease in $\mathrm{da} / \mathrm{dN}$ was observed for $\mathrm{P}_{\mathrm{H} 2 \mathrm{O}}$ values of $18,1.8$, and $4 \mathrm{~Pa}$ between a $\Delta \mathrm{K}$ of 8-7 MPa $\sqrt{\mathrm{m}}$. The da/dN minima occurred for a $\Delta \mathrm{K}$ range of 6.7 to $7.0 \mathrm{MPa} \sqrt{\mathrm{m}}$, before systematically increasing with decreasing $\Delta K$. The threshold transition regime behavior for 2199-T86 occurred at a higher range of $\mathrm{P}_{\mathrm{H} 2 \mathrm{O}}$ and stress intensity range than 7075-T651. A direct comparison between the data from the 7075-T651 and 2199-T86 samples is complicated by the fact that the 2199-T86 sidegrooved samples have different water-molecular transport distances. For $\mathrm{P}_{\mathrm{H} 2 \mathrm{O}}$ below $38 \mathrm{~Pa}$, a strong environmental dependence between da/dN and $\mathrm{P}_{\mathrm{H} 2 \mathrm{O}}$ was observed. Figure 25 plots the $\mathrm{da} / \mathrm{dN}$ minimum value in the TTR as a function of $\mathrm{P}_{\mathrm{H} 2 \mathrm{O}}$ for $7075-\mathrm{T} 651$ and 2199-T86. Both the 7075-T651 and 2199-T86 data demonstrate that the magnitude of the drop increases with decreasing $\mathrm{P}_{\mathrm{H} 2 \mathrm{O}}$. The mechanisms responsible for the da/dN vs $\Delta \mathrm{K}$ differences in the threshold transition regime between 7075-T651 and 2199-T86 will be addressed in detail in the Discussion.

\subsubsection{Fracture Surface Morphology}

Each 2199-T86 fracture surface produced by $\Delta \mathrm{K}$-shed loading was examined through similar low magnification optical microscopy and SEM fractography (Figure 26). For all decreasing $\Delta \mathrm{K}$ experiments between $4 \mathrm{~Pa}$ and $0.5 \mathrm{~Pa}$ (Figure 26b-26d), the specimen's fracture surface showed a transition from a smooth fracture surface to a rough surface in the middle. The change in morphology correlates with the threshold transition region for the $18,1.8$, and $4 \mathrm{~Pa}$ 
exposures and is shown for the fracture surface of a L-T specimen stressed at $\mathrm{P}_{\mathrm{H} 2 \mathrm{O}}=1.8 \mathrm{~Pa}$ in Figure 27. Lines of matching style between the optical fractograph and the da/dN plot correspond to the compliance indicated crack front location at the $\Delta \mathrm{K}$ of interest. The decrease in the da/dN correlates to the increased roughness portion of the fracture surface. This transition from a smooth to rough fracture surface occurred for the $0.5 \mathrm{~Pa}$ sample, but is not reflected in the corresponding growth rates seen in Figure 22 where da/dN systematically decreased with decreasing $\Delta \mathrm{K}$. For testing completed at a $\mathrm{P}_{\mathrm{H} 20}>18 \mathrm{~Pa}$, the specimen's fracture surface had a nearly uniformed macroscopic fracture pattern for the entirety of the K-shed loading segment (Figure 26f-26g). The roughness transition seen for the exposures between 0.5 and $18 \mathrm{~Pa}$ suggests a potentially more complex mechanism transition that depends on $\Delta \mathrm{K}$ and the environment.

SEM fractography of the K-shed fracture surfaces was performed for all exposures looking at key $\Delta \mathrm{K}$ values before, during, and after the threshold transition region as shown previously for 7075-T651. The 2199-T86 fractographic surfaces showed faceted cracking (SBC-like features) in inert environments for all $\Delta \mathrm{K}$ (Figure 28). At higher $\Delta \mathrm{K}$ and high humidity (Figure 28b and 28d), the fracture surface transitioned to a combination of SBC and transgranular cracking. The fracture surface morphology varied with water vapor pressure ranging from UHV to relative high humidity (2668 Pa) (Figures 29 and 30) when compared at $\Delta \mathrm{K}=7 \mathrm{MPa} \sqrt{\mathrm{m}}$. The fraction of faceted features systematically decreased with increasing water vapor pressure. SBC-like features were first observed at $\mathrm{P}_{\mathrm{H} 2 \mathrm{O}}=4 \mathrm{~Pa}$ (Figures $29 \mathrm{~d}$ and 30d) with increasing occurrence as $\mathrm{P}_{\mathrm{H} 2 \mathrm{O}}$ decreased to UHV. At higher exposures (>4 Pa, Figures $29 \mathrm{e}-29 \mathrm{~g}$ and 30e-30g), the crack morphology became more transgranular; typically associated with $\mathrm{H}$-assisted cracking [1]. The fracture surface morphologies before the threshold transition regime showed transgranular fracture patterns for exposures greater than $1.8 \mathrm{~Pa}$ at $\Delta \mathrm{K}=9 \mathrm{MPa} \sqrt{\mathrm{m}}$ (Figure 31 ). A small degree of $\mathrm{SBC}$ was found 
at UHV and $0.5 \mathrm{~Pa}$ at $\Delta \mathrm{K}=9 \mathrm{MPa} \sqrt{\mathrm{m}}$ (Figure $31 \mathrm{a}-31 \mathrm{~b}$ ). The fracture surface morphologies after the threshold transition regime $\Delta \mathrm{K}=5 \mathrm{MPa} \sqrt{\mathrm{m}}$ showed transgranular fracture patterns for high humidity exposures (Figure $32 \mathrm{f}-32 \mathrm{~g}$ ). SBC-like features were first observed at $\mathrm{P}_{\mathrm{H} 2 \mathrm{O}}=18 \mathrm{~Pa}$ (Figure 32e) with increasing occurrence as $\mathrm{P}_{\mathrm{H} 2 \mathrm{O}}$ decreased to UHV.

The fracture surface morphology in the threshold transition regime for the 1.8 and $4 \mathrm{~Pa}$ samples showed a transition from transgranular fracture to SBC-like morphology that was consistent with the low-magnification optical images (Figures 26 and 27). Figure 33 shows SEM images of the fracture surface for the $1.8 \mathrm{~Pa}$ exposure at specific $\Delta \mathrm{K}$ across the threshold transition regime. Transgranular fracture patterns were seen at $\Delta \mathrm{K}=9 \mathrm{MPa} \sqrt{\mathrm{m}}$ (Figure 33a), which corresponded to growth rates slightly before the onset of the dip in da/dN. SBC-like features were present at $\Delta \mathrm{K}=7$ and $6 \mathrm{MPa} \sqrt{\mathrm{m}}$, which corresponded to the minimmum of the dip in $\mathrm{da} / \mathrm{dN}$ (Figure $33 \mathrm{~b}$ and $33 \mathrm{c}$ ). As the $\Delta \mathrm{K}$ decreased to $5 \mathrm{MPa} \sqrt{\mathrm{m}}$ (Figure $33 \mathrm{~d}$ ), the crack surface morphology showed decreasing amounts of SBC-like features. Previous work investigating the fatigue behavior of peak-aged AA2090 (L-T) in various environments [54] supports the expected fracture morphology differences and complexity seen when comparing 2199-T86 to 7075-T651. In AA2090, SBC was found in inert environments at all $\Delta \mathrm{K}$ and relatively flat crystallographic $\{100\}$ cracking was seen in H-producing environments. While different alloy classes, both AA2090 and 2199-T86 contain the same shearable $\delta$ '-phase $\left(\mathrm{Al}_{3} \mathrm{Li}\right)$ particle which can dictate slip behavior $[40$, $44,49,50]$. The mechanisms responsible for the fracture surface morphology differences between 7075-T651 and 2199-T86 and the effect on the threshold transition regime will be addressed in the Discussion. 


\section{Discussion}

Experimental results reinforced the expected strong water vapor dependence of $\mathrm{da} / \mathrm{dN}$ across a wide range of $\Delta \mathrm{K}$ for $7075-\mathrm{T} 651$ and 2199-T86. Increasing the water vapor pressure resulted in increased $\mathrm{da} / \mathrm{dN}$ at all $\Delta \mathrm{K}$ levels and a substantial reduction in the cracking threshold [1]. This behavior is well known and has been addressed based on hydrogen environment embrittlement $[1,18-21,55]$. Additionally, a novel effect of water vapor on the $\Delta \mathrm{K}$ dependence of $\mathrm{da} / \mathrm{dN}$ in the near-threshold regime was exhibited for low water vapor exposures [1]. These data provide a solid foundation for incorporation into engineering prognosis to predict cracking in these high altitude environments, but further mechanistic and scientific knowledge of the nearthreshold regime behavior is needed before final integration.

This Discussion first examines the effect of surface roughness on molecular transport from the crack mouth to the crack tip. Specifically, the interaction between molecular flow and the degree of roughness and the subsequent effect on da/dN will be addressed. The crack front evolution in the threshold transition regime will be determined as a means to evaluate the proposed molecular transport based explanation for the threshold transition regime. Next, the post minima behavior for 7075-T651 will be investigated as it relates to the proposed turbulent mixing hypothesis. The environmental fatigue crack growth and near-threshold regime behavior of 2199T86 will also be examined. Finally, the context of how these experimental results and understanding can be applied to engineering fatigue life prognosis will be discussed. 


\subsection{The Effect of Surface Roughness on Molecular Transport}

\subsubsection{Molecular transport mechanisms}

The crack growth kinetics and rate controlling reactions occurring within a crack depend on the local environment, which is determined by the mass transport of water vapor molecules between the bulk solution and the crack tip [56]. Mass transport, also described as the molecular flow, from the crack mouth to crack tip will occur by the combination of fluid flow and diffusion [23]. As the crack grows, the increasing diffusion distance and the limited space within the crack can result in a limitation in the movement of the water vapor molecules from the crack mouth to the crack tip [40]. Wei and coworkers proposed that the $\mathrm{P}_{\mathrm{H} 2 \mathrm{O}}$ at the crack tip is lower than the bulk $\mathrm{P}_{\mathrm{H} 2 \mathrm{O}}$ due to the impeded diffusional (Knudsen) flow of water molecules interacting with crack walls. They modeled the crack growth in this diffusion-based regime as $[1,10,18,30-31,33-35$, 40]:

$$
\left(\frac{d a}{d N}\right)_{c f}=\frac{P_{H_{2} O}}{f}\left[436 \frac{\beta}{\alpha N} f(R) \frac{d \sigma_{y s}^{2}}{k T E^{2}}\left(\frac{T}{M}\right)^{\frac{1}{2}}\right]\left(\frac{d a}{d N}\right)_{c f-S a t}
$$

where $\mathrm{f}$ is frequency, $\mathrm{N}$ is the density of surface reaction sites $\left(10^{19} \mathrm{Al}\right.$ atoms $\left./ \mathrm{m}^{2}\right)$ in the $\mathrm{Al}-\mathrm{H}_{2} \mathrm{O}$ reaction to produced a surface layer of absorbed-atomic $\mathrm{H}, \mathrm{k}$ is the Boltzmann constant, $\mathrm{d}$ is distance from the crack tip at which the crack opening displacement (COD; used to approximate the flow channel dimensions) is calculated, $\mathrm{T}$ is absolute temperature, E is Young's modulus, $\mathrm{M}$ is the molecular weight of water vapor, $f(R)$ is a function of stress ratio, and $\alpha$ and $\beta$ are empirical constants related to surface roughness of the crack increment formed during the previous cycle and flow geometry, respectively [1]. In this diffusion-based regime, the growth rates are directly related to the $\mathrm{P}_{\mathrm{H} 2 \mathrm{O}} / f$ and the surface roughness of the crack face ( $\alpha$ and $\beta$ ) which suggests that variations in the crack surface roughness could have differing effects on subsequent growth rates. 
The impeded molecular transport hypothesis proposes that the onset of the threshold transition minimum in $\mathrm{da} / \mathrm{dN}$ is caused by roughness-induced changes in the mass transport behavior that governs the $\mathrm{P}_{\mathrm{H} 2 \mathrm{O}}$ at the crack tip [1]. This hypothesis applies when the molecular transport of water vapor molecules from the crack mouth to crack tip is governed by diffusional (Knudsen) flow. Turnbull proposed an alternative laminar flow transport mechanism, called advection, to describe the movement of molecules. In fatigue cracking, the cyclic movement of the crack walls helps to induce bulk flow from the crack mouth to the crack tip [56]. Turnbull defined a critical crack distance $\left(l_{\text {crit }}\right)$ where molecular transport switches from being diffusion supplied to advection supplied for deeper cracks. When the molecular transport is advection supplied at a crack length $>l_{\text {crit, }}$, the molecular flow can be viewed as diffusion limited. This critical distance $\left(l_{\text {crit }}\right)$ is the location where diffusion and advection are equally effective transport processes for an idealized smooth-surface trapezoidal crack of blunted tip opening displacement and elastic mouth opening displacement $[1,57,58]$ :

$$
l_{\text {crit }} \approx\left(\mathrm{D}_{\mathrm{H} 2 \mathrm{O}} / f\right)^{1 / 2} /\left(1-\mathrm{R}^{1 / 2}\right)
$$

where $\mathrm{D}_{\mathrm{H} 2 \mathrm{O}}$ is the diffusivity of water vapor molecules. The $\mathrm{D}_{\mathrm{H} 2 \mathrm{O}}$ for pure water vapor is 0.28 $\mathrm{cm}^{2} / \mathrm{s}$ at $23^{\circ} \mathrm{C}$ and $100 \mathrm{kPa}$ pressure [59]. D $\mathrm{H}_{2 \mathrm{O}}$ decreases with increasing water vapor pressure proportional to $1 / \mathrm{P}_{\mathrm{H} 2 \mathrm{O}}$, in addition to decreasing for restricted channel volumes with dimensions that fall below the mean-free path of the molecules as given by the Knudsen flow [1, 57-62]. For loading conditions typical of the threshold transition regime, $\mathrm{C}(\mathrm{T})$ specimens have a crack opening displacement that is on average orders of magnitude smaller than the mean-free path for water molecules which results in a modified $\mathrm{D}_{\mathrm{H} 2 \mathrm{O}}[1,58]$. The $\mathrm{D}_{\mathrm{H} 2 \mathrm{O}}$ modified for diffusional (Knudsen) flow in an idealized channel with a height equal to the average $\operatorname{COD}(5 \mu \mathrm{m}$ for $\mathrm{R}=0.5$ testing $)$ is 8 $\mathrm{cm}^{2} / \mathrm{s}[1,63]$. Based on these values and presented previously [1], the $l_{\text {crit }}$ value was found to equal 
$43 \mathrm{~mm}$ for K-shed/constant $\mathrm{R}$ testing. The crack depth before the onset of the threshold transition regime was $22-30 \mathrm{~mm}$ ahead of the $\mathrm{C}(\mathrm{T})$ specimen notch root $\left(<l_{\text {crit }}=43 \mathrm{~mm}\right)$ suggesting that the threshold transition regime is within the diffusion supplied region of molecular transport.

As seen in Wei's equation for crack growth rates in diffusion-based regimes, the growth rates are directly related to the $\mathrm{P}_{\mathrm{H} 2 \mathrm{O}} / f$ and the surface roughness of the crack face $(\alpha$ and $\beta)$. As the crack surface becomes rougher with increasing proportions of SBC, the diffusion-based Knudsen flow of water molecules becomes increasingly impeded; resulting in decreased $\beta$ and thus decreased local $\mathrm{P}_{\mathrm{H} 2 \mathrm{O}}$ and da/dN [30, 31, 33]. Researchers have found that increasing crack wake roughness, and by extension, crack asperity topography, will result in a reduction in the effective crack opening for the molecular flow path [1, 64-66]. Increasing the height and decreasing the spacing between crack wake surface features has shown to increase the impedance of the molecular (Knudsen) flow through micro-channels [1, 64-68]. This roughness induced impeded flow would augment the likely contributions of roughness induced closure to cause a decrease in the da/dN.

Previous work [1] has proposed that when a critical span and/or magnitude of roughness is reached, the water vapor transport mechanism changes from diffusion or advection based flow to a fully convective mixing due to crack wake asperity contact. The asperity contact helps to initiate turbulent flow that imparts momentum into the crack tip water vapor molecules. These enhancedflow molecules raise the $\mathrm{P}_{\mathrm{H} 2 \mathrm{O}}$ at the crack tip back to the bulk $\mathrm{P}_{\mathrm{H} 2 \mathrm{O}}$. This hypothesis agrees with the ideas presented by Somerday and coworkers who examined the flow of $\mathrm{H}_{2}$ gas along a flat crack without faceted roughness [1,58]. After studying the flow properties of $\mathrm{H}_{2}$ in relation to its low Reynolds number, they recognized the possibility of turbulent flow occurring due to crack wake asperity contact helping to equilibrate the crack tip pressure $[1,58]$. Taunt and Charnock 
proposed that the pumping action of $\mathrm{C}(\mathrm{T})$ specimens may accentuate environmental effects by increasing the supply of species to the crack tip; specifically suggesting that large, frequent load cycles would result in increased fluid turbulence that would enhance mixing [59]. A quantitative analysis of the occurrence of crack wake asperity contact will be presented later, but there remains a need to quantify the effect of crack wake roughness on the da/dN behavior; specifically how it relates to impeding or enhancing molecular transport from the crack mouth to the crack tip.

\subsubsection{Initial influences of crack wake roughness on $\mathrm{da} / \mathrm{dN}$ behavior $(<5 \mathrm{~mm}$ of crack growth)}

The interaction of the bulk $\mathrm{P}_{\mathrm{H} 2 \mathrm{O}}, \Delta \mathrm{K}$, and crack wake roughness can have differing effects on crack closure and water vapor transport in the crack wake leading to deviations from the unique $\mathrm{da} / \mathrm{dN}$ versus $\Delta \mathrm{K}$ relationship for constant intermediate water vapor exposures, as established in Figure 1 [1]. A quantitative interpretation of the interaction between surface roughness and molecular transport is difficult to reach using the K-shed experiments (Figure 1), because the load history, crack wake roughness, and $\Delta \mathrm{K}$ are all varying simultaneously. In order to isolate and target this relationship, a test method was designed to change the crack wake roughness while keeping constant the bulk $\mathrm{P}_{\mathrm{H} 2 \mathrm{O}}, \Delta \mathrm{K}$, and initial crack length. The roughness spans were selected to represent a crack tip that would experience impeded-molecular $(0.5 \mathrm{~mm})$ or enhanced $(3 \mathrm{~mm})$ turbulent convective flow based on the molecular transport hypothesis which presented the idea that a critical magnitude of roughness is needed to initiate turbulent mixing and enhance growth rates [1]. Based on the molecular transport hypothesis, the $0.5 \mathrm{~mm}$ of crack wake roughness would represent a subcritical amount of roughness that would initially impede the molecular transport and retard crack growth rates. The $3 \mathrm{~mm}$ of crack wake roughness experiment would represent the condition where the magnitude of roughness is large enough to encourage turbulent convective 
mixing resulting in enhanced crack growth rates. This information will be inferred by the da/dN behavior; elevated levels of da/dN will suggest continued enhanced crack tip $\mathrm{P}_{\mathrm{H} 2 \mathrm{O}}$, while a consistent or steady state $\mathrm{da} / \mathrm{dN}$ will suggest a diminished effect of turbulent convective mixing. The results of these varying roughness span experiments will be critical to understanding (1) whether subcritical spans of roughness lead to impeded growth and longer spans will lead to enhanced crack growth, and (2) the extent to which crack wake roughness will continue to influence the crack growth behavior as the crack tip moves further away from these features.

For each crack wake roughness experiment, the da/dN behavior was plotted as a function of crack length with the corresponding fracture surface underneath (Figures 14 and 16). The growth rate for $\Delta \mathrm{K}=5 \mathrm{MPa} \sqrt{\mathrm{m}}$, estimated from the $\mathrm{K}$-shed experiment (Figure 1), is da/dN $=4.0$ $\mathrm{x} 10^{-6} \mathrm{~mm} /$ cycle and was used as the comparison to determine whether the roughness experiments were experiencing impeded or enhanced growth rates. The da/dN behavior of the $0.5 \mathrm{~mm}$ of crack wake roughness experiment was initially impeded $\left(\mathrm{da} / \mathrm{dN}<4.0 \times 10^{-6} \mathrm{~mm} /\right.$ cycle) for $2 \mathrm{~mm}$ before increasing to reach a steady state $\mathrm{da} / \mathrm{dN}=5.0 \times 10^{-6} \mathrm{~mm} /$ cycle that was maintained for $2 \mathrm{~mm}$ of crack growth. The da/dN behavior for the first $4 \mathrm{~mm}$ of crack growth agreed well with the molecular transport hypothesis that suggested the crack wake roughness would impede water vapor transport from the crack mouth to the crack tip. The ACR and ASTM $2 \%$ ratio were plotted to account for the possible effects of crack closure on da/dN (Figure 14c). The highest contribution of crack closure (closure ratio $=0.8$ ) would reduce the $\Delta \mathrm{K}_{\text {eff }}$ from 5 to $4.0 \mathrm{MPa} \sqrt{\mathrm{m}}$, corresponding to a steady state $\mathrm{da} / \mathrm{dN}=1.0 \times 10^{-6} \mathrm{~mm} /$ cycle. The impeded growth rates observed for the first millimeter of crack growth for the $0.5 \mathrm{~mm}$ sample start at da/dN $=1.0 \times 10^{-7}$; rapidly increasing before reaching a steady state $\mathrm{da} / \mathrm{dN}$. While the initial lower growth rate trend is consistent with the ACR ratio change, an additional influence of environmental cracking is suggested since the 
magnitude of the $\Delta \mathrm{K}$ change is not sufficient enough to explain the initial impedance in growth rates. Additionally, at longer crack lengths (> $22 \mathrm{~mm})$ the changes in the ASTM $2 \%$ and ACR ratios do not directly correspond to changes in the $\mathrm{da} / \mathrm{dN}$ behavior of the $0.5 \mathrm{~mm}$ sample indicating that crack closure is not the controlling mechanism.

The $\mathrm{da} / \mathrm{dN}$ behavior of the $3 \mathrm{~mm}$ of crack wake roughness experiment was initially impeded $\left(\mathrm{da} / \mathrm{dN}<4.0 \times 10^{-6} \mathrm{~mm} /\right.$ cycle) for $2 \mathrm{~mm}$ of crack growth before increasing to a maximum $\mathrm{da} / \mathrm{dN}=1.0 \times 10^{-5}$, signifying enhanced growth rates when compared to the $\Delta \mathrm{K}$-shed growth rate. Again, the ACR and ASTM 2\% ratio were plotted to account for the possible effects of crack closure on da/dN (Figure 16c). The $3 \mathrm{~mm}$ of roughness experiment also produced a $20 \%$ reduction in the $\Delta \mathrm{K}_{\text {eff }}$ corresponding to an adjusted steady state da/dN $=1.0 \times 10^{-6} \mathrm{~mm} /$ cycle. Similar to the $0.5 \mathrm{~mm}$ sample, the low da/dN values correlate with similar trends in the ASTM $2 \%$ and ACR plots for the $3 \mathrm{~mm}$ sample, however the magnitude of the $\Delta \mathrm{K}$ reduction would not fully describe the initial impedance in $\mathrm{da} / \mathrm{dN}$ and the trends for longer cracks do not directly correspond to changes in the $\mathrm{da} / \mathrm{dN}$ behavior.

These results tend to generally support the hypothesis that shorter spans of crack wake roughness will impede subsequent crack growth due to Knudsen flow limitation, while longer spans can enhance crack growth due to turbulent convection. However, the initial impedance in the longer span test is only partially attributed to closure; the remaining cause of this reduction is not known and may compromise this conclusion. The fracture surface corresponding to the region of crack growth prior to the onset of the first fluctuations in $\mathrm{da} / \mathrm{dN}$ and surface roughness was examined to investigate the initial impedance before increasing to elevated crack growth rates for the $3 \mathrm{~mm}$ sample (Figure 17). For this initial region, the fracture pattern from the sides (Figure 17 B.1 and B.3) show cracking inwardly from the sides. The fracture surface in the middle of the 
sample at the same crack length showed a crack progression from left to right (Figure 17 B.2). The macroscopic overview of the fracture surface for this region suggests that cracking in from the sides may be dominating over crack growth in the notch direction. Cracking from the sides would result in an irregular crack front that could alter the relationship between the recorded compliance and the applied load to achieve the programed $\Delta \mathrm{K}$ value.

One theory to explain the increase in $\mathrm{da} / \mathrm{dN}$ from being initially impeded to elevated in the $3 \mathrm{~mm}$ sample could be related to the mechanics associated with an irregular crack. Specifically, an irregular crack front (similar to that reported in Figure 11) would result in a higher $\Delta \mathrm{K}$ driving force at the center portion of the specimen. The increased driving force could combine with high levels of constraint in the center of the specimen, resulting in enhanced $\mathrm{da} / \mathrm{dN}$ in order for the center crack portion to "catch up" to the environmentally enhanced cracking along the sides. Based on this theory and the molecular transport hypothesis, the proposed crack front progression during the first $4 \mathrm{~mm}$ of crack growth following the crack wake roughness is illustrated in Figure 34 for the 0.5 and $3 \mathrm{~mm}$ roughness experiments. The crack wake roughness performed at UHV is indicated by the blue lines and the green lines represent the crack front as it progresses left to right corresponding to the numbers above each continuous crack front. As the crack wake roughness impedes molecular transport in the notch direction, environmental contributions from the crack faces results in faster cracking inwardly than in the notch direction. As the irregular crack front becomes more pronounced, the slower cracking portion in the center experiences a growing amount of applied load and an increased potential for further contributions of $\mathrm{H}$ diffusion from the crack faces (crack fronts 2-3 in Figure 34a and 34b). Both of these factors contribute to enhanced growth rates as the middle portion of the crack front catches up to the sides and a regular crack front is resumed (crack front 4 in Figure 34a and 34b). The differences in the crack front 
progression between the two roughness experiments is a result of the $3 \mathrm{~mm}$ of roughness completely impeding diffusion from the notch direction and diffusion inwardly from the sides dominating, whereas the $0.5 \mathrm{~mm}$ of crack wake roughness only partially impedes diffusion from the notch direction resulting in a combination of diffusion inwardly from the sides and from the notch.

These constant $\Delta \mathrm{K}, \mathrm{P}_{\mathrm{H} 2 \mathrm{O}} / f$ experiments serve to show that the interaction between the roughness on the fracture surface and the molecular transport can result in an order of magnitude change in growth rates directly following crack growth of increased roughness. Additionally, the fractography suggests an environmentally driven irregular crack front that is driven by the interaction of molecular transport, closure, asperity based mixing, and position dependent $\Delta \mathrm{K}$. These findings will be extended upon through examining the crack front evolution in the threshold transition regime during K-shed loading and the effect of the initial roughness span on the da/dN behavior vs. crack length as the crack continues to grow will be addressed in depth in Section 4.3.2.

\subsection{Crack Front Evolution in the Threshold Transition Regime}

The results of the crack wake roughness experiments suggested that different magnitudes of roughness could have an effect on crack front evolution and subsequent da/dN behavior. This finding indicates that the observed surface roughness in the $0.2 \mathrm{~Pa}$ and $0.5 \mathrm{~Pa}$ exposures (Figure $3 \mathrm{a}$ and $\mathrm{b}$ ) could also be producing an irregular crack front during the threshold transition region. To that end, initial fracture surface morphology for low exposure $\Delta \mathrm{K}$-shed testing in the postminimum region (Figure $3 \mathrm{a}$ and $\mathrm{b}$ ) showed the possibility of a complex crack front where environmentally enhanced transgranular fractures dominates inwardly from specimen surfaces and 
SBC continues in the center of the specimen [1]. Figure 11 shows the crack front evolution during the threshold transition region with marker-band loading sequences at $\Delta \mathrm{K}$ of 5,4 , and $3 \mathrm{MPa} ل_{\mathrm{m}}$. The calculated and experimental crack fronts for $\Delta \mathrm{K}=5 \mathrm{MPa} \sqrt{\mathrm{m}}$ are similar in location and shape suggesting a strong correlation between the compliance calculated crack front and the experimental data at the start of the threshold transition region. At this location, there is no SBC in the crack wake to initially impede the molecular transport. As $\Delta \mathrm{K}$ continues to decrease, the experimental crack fronts for $\Delta \mathrm{K}=4$ and $3 \mathrm{MPa} \sqrt{\mathrm{m}}$ become more irregular, suggesting cracking inwardly from the crack faces is dominating over crack progression in the middle of the sample. Since the half-thickness dimension is small compared to the crack depth it is reasonably assumed that the pertinent molecular transport distance is inward from the specimen faces. For diffusionbased flow, the crack wake roughness will further decrease the molecular transport from the notch direction. As such the shorter flow distances from the specimen surfaces facilitates hydrogen enhanced cracking (thus faster $\mathrm{da} / \mathrm{dN}$ ) near the edges as opposed to the specimen center that is starved of crack tip $\mathrm{P}_{\mathrm{H} 2 \mathrm{O}}$ due to the longer and more impeded diffusional flow [1]. As the crack surface near the specimen face becomes rougher, the diffusion based flow becomes more difficult, and the transport from the surface to the center is harder. The resulting reduction in $\mathrm{P}_{\mathrm{H} 2 \mathrm{O}}$ at the center results in retarded crack growth in the center compared to faster cracking on the edges.

From a purely mechanics perspective, researchers have investigated the influence of free surfaces on fatigue crack behavior and resulting variations in the stress intensity distribution [6971]. Work by Lin and Smith showed that during crack progression, the presence of free surfaces made it increasingly difficult for a constant, i.e., iso-K profile to be maintained across the thickness direction of the sample [71, 79]. Garcia-Manrique and coworkers used a model to numerically evaluate the stress intensity distribution along the thickness for an Al 2024-T35 C(T) specimen 
under Mode I nominal loading. They found that curvature of the crack front (where the crack flanks lagged behind) could result from a non-uniform $\mathrm{K}$ distribution along the thickness of the specimen. They postulated two mechanisms that would contribute to an uneven load distribution along the crack front: (1) crack closure effects near the free surface of the specimen resulting in a smaller $\Delta \mathrm{K}_{\mathrm{eff}}$ and slower growth rates near the exterior and (2) a decrease in the plastic zone size in regions closer to the surface resulting in a smaller $\Delta \mathrm{K}_{\text {eff }}$ near the sides than in the middle [69]. Both these mechanisms would imply an irregular crack front shape that is retarded on the sides when compared to the middle of the sample. The experimental crack fronts observed at $\Delta \mathrm{K}=4$ and $3 \mathrm{MPa} \sqrt{\mathrm{m}}$ for the $0.5 \mathrm{~Pa}$ exposure show retarded growth in the center when compared to the exteriors of the sample, suggesting that the plastic zone and crack closure effects are not the controlling mechanisms for this behavior, but instead that this irregular crack front is mechanistically controlled by the environment and the molecular transport from crack mouth to crack tip.

The irregular crack shape observed at $\Delta \mathrm{K}=4$ and $3 \mathrm{MPa} \sqrt{\mathrm{m}}$ results in non-uniform stress intensity values along the crack front (which would be compounded by inaccuracies in compliance readings and the applied load due to the assumption of a straight crack front during testing). As the crack shape becomes more irregular, compliance readings (which assume a straight crack front) may erroneously report the crack length. It is not clear how such an irregular crack front will influence the compliance measurement, but if the true crack length is longer than crack size calculated via compliance, an erroneously high $\Delta \mathrm{K}$ load would result. Figures 12 and 13 plotted the dependence of $\mathrm{da} / \mathrm{dN}$ on $\Delta \mathrm{K}$ and $\mathrm{P}_{\mathrm{H} 2 \mathrm{O}}$ for the L-T orientation of 7075-T651 measured under decreasing $\Delta \mathrm{K}(\mathrm{R}=0.5, f=20 \mathrm{~Hz})$; specifically highlighting the low $\Delta \mathrm{K}$ behavior of the 0.5 and $0.2 \mathrm{~Pa}$ exposures. The rapid increase or spike in $\mathrm{da} / \mathrm{dN}$ two orders of magnitude occurred for a 
$\Delta \mathrm{K}$ range of 3.7 to $4.0 \mathrm{MPa} \sqrt{\mathrm{m}}$. After the spike, growth rates returned to systematically decreasing $\mathrm{da} / \mathrm{dN}$ with decreasing $\Delta \mathrm{K}$. The irregular crack front developed during the threshold transition regime likely causes this spike in the data. Similar to the roughness experiments, the proposed crack front progression during the threshold transition regime of the $0.5 \mathrm{~Pa} \mathrm{~K}$-shed experiment is shown in Figure 35. Based on the interaction between an irregular crack front and the molecular transport hypothesis; as the crack wake roughness impedes molecular transport in the notch direction (crack fronts 1-2 in Figure 35), an environmental contribution from the crack faces results in faster cracking inwardly than cracking in the notch direction. As the crack shape becomes more irregular (crack fronts 3-5 in Figure 35), the driving force at the center would continue to increase resulting in an upswing in growth rates, thus the spike in $\mathrm{da} / \mathrm{dN}$, as the middle portion of the crack front catches up to the sides and a regular crack front is resumed (crack fronts 6-7 in Figure 35) at the conclusion of the TTR. This behavior is consistent with the results of Branco et al. that showed that the $\mathrm{K}$ profile is dependent of the geometry of the component and the applied load which can ultimately affect crack shape evolution [82]. The K distribution along a crack front was the driving force for crack shape variations and that a crack shape with a lagging center will result in a higher applied stress intensity at that center [82]. The optical fractograph of the $0.5 \mathrm{~Pa}$ exposure showed fully transgranular cracking at $\Delta \mathrm{K}=3 \mathrm{MPa} \sqrt{\mathrm{m}}$ corresponding to the return of the systematically decreasing da/dN with decreasing $\Delta \mathrm{K}$.

The subsequent spike in da/dN after the TTR was also observed for the $0.2 \mathrm{~Pa}$ exposure and plotted in Figure 13. The spike in da/dN observed for the $0.2 \mathrm{~Pa}$ sample had a similar magnitude as the $0.5 \mathrm{~Pa}$ sample, but occurred for a $\Delta \mathrm{K}$ range of 3.3 to $3.7 \mathrm{MPa} \sqrt{\mathrm{m}}$. Unlike the 0.5 Pa sample, the growth rates after the spike did not systematically decrease, but instead fluctuated with decreasing $\Delta \mathrm{K}$, indicating a high degree of scatter. Both these exposures exhibited similar 
TTR behavior, but showed different da/dN behavior in the post-minima regime suggesting that the magnitude of roughness and the morphology of the subsequent cracking along the side of the specimen can affect post-TTR da/dN behavior. The fluctuations may be attributed to either of two things. First, if a fully regular crack front were not achieved, a simple mechanics explanation would suggest that the non-crack area in the center would be experiencing a high driving force and would quickly crack in response to the varying stress intensity along the non-uniform crack front. The fluctuations observed for $0.2 \mathrm{~Pa} \mathrm{~K}$-shed results in the post TTR regime may be due to this process repeating to various degrees. Second, the $0.5 \mathrm{~Pa}$ exposure had a smaller roughness span and less crack asperity roughness when compared to the $0.2 \mathrm{~Pa}$ exposure (Tables 1 and 5). Based on the molecular transport hypothesis, a higher magnitude of roughness may affect crack tip $\mathrm{P}_{\mathrm{H} 2 \mathrm{O}}$ for longer periods of crack growth. This would suggest that the scatter in growth rates after the TTR for $0.2 \mathrm{~Pa}$ sample could be a result of the irregular crack front experiencing enhanced $\mathrm{P}_{\mathrm{H} 2 \mathrm{O}}$ for a longer period of crack growth when compared to the $0.5 \mathrm{~Pa}$ sample.

In summary, the K-shed marker-band experiment showed that cracking after the bands of roughness will initiate at the specimen surface and propagate as an elliptical crack toward the center and along the crack flanks. This behavior is uniquely explained by a molecular transport mechanism, thus an environmentally based effect. Further, this reinforces the assertion that the through thickness direction is the pertinent molecular transport distance, particularly for post roughness cracking where the SBC morphology further impedes crack flow in the cracking direction. Molecular transport in the through thickness dimension controls the environmental influence, particularly in the TTR regime. The non-uniform crack front that is developed has varying stress intensity along the crack front. The spike in growth rates and the change in fracture morphology suggest a mechanics based "fast" cracking mechanism that is responsible for the 
observed spike and fluctuations in the post TTR for the 0.2 and $0.5 \mathrm{~Pa}$ K-shed tests. Errors in the compliance based interpretation and the K-solution associated with the irregular crack front likely also contribute to the spike behavior. A better understanding of the controlling mechanisms in the post-minima regime is needed and will be examined next before a strong conclusion can be made about how long the surface roughness and irregular crack front will affect $\mathrm{da} / \mathrm{dN}$ behavior after the threshold transition regime.

\subsection{Post Minima Regime Behavior for 7075-T651}

The results of previous experimentation have shown that the magnitude of surface roughness can have an effect on crack front evolution and da/dN behavior at low exposures and intermediate $\Delta \mathrm{K}$, but there remains a need to explain and quantify the controlling mechanisms for the increase in crack growth rates following the da/dN minima. Specifically, investigating why the fracture surface transitions back to fully transgranular facture and increasing da/dN, instead of continuing to slip band crack. The Burns et al. paper proposed three hypotheses to explain the continued elevated crack growth kinetics after the crack grows 10-15 mm beyond the crack wake roughness: (1) for low $\mathrm{P}_{\mathrm{H} 2 \mathrm{O}}$ exposures, $\mathrm{SBC}$ is only active at intermediate $\Delta \mathrm{K}$ and becomes limited at low $\Delta \mathrm{K},(2)$ discontinuous cracking shifts the relevant exposure parameter out of the molecular flow controlled regime and allows more time/cycles for molecular transport, or (3) a continued strong contribution of turbulent mixing is maintained keeping elevated $\mathrm{P}_{\mathrm{H} 2 \mathrm{O}}$ [1].

The first hypothesis proposed that the transition back to fully transgranular cracking is due to $\mathrm{SBC}$ being active only at intermediate $\Delta \mathrm{K}$ and limited at low $\Delta \mathrm{K}$ for low $\mathrm{P}_{\mathrm{H} 2 \mathrm{O}}$ exposures. To test the validity of this hypothesis, a constant $\Delta \mathrm{K}=3 \mathrm{MPa} \sqrt{\mathrm{m}}\left(\mathrm{K}_{\max }=6 \mathrm{MPa} \sqrt{\mathrm{m}}, f=20 \mathrm{~Hz}, \mathrm{R}=0.5\right)$ test was completed to examine the ability of 7075-T651 to slip band crack at low $\Delta \mathrm{K}$ and exposures 
with no load effects in the fracture surface. The lack of SBC at $\Delta \mathrm{K}$ above $6 \mathrm{MPa} \sqrt{\mathrm{m}}$ transitioning to SBC at intermediate $\Delta \mathrm{K}(4-5 \mathrm{MPa} \sqrt{\mathrm{m}})$, is postulated to be caused by the ability for cross-slip at higher $\Delta \mathrm{K}[1]$. Specifically, in UHV and low $\mathrm{P}_{\mathrm{H} 2 \mathrm{O}}$ environments, there are many active slip systems at higher $\Delta \mathrm{K}(7-9 \mathrm{MPa} \sqrt{\mathrm{m}})($ Figures 6 and 9) and sufficient local driving force to enable cross-slip and facilitate a non-crystallographic crack path along the notch direction [1]. However at intermediate $\Delta \mathrm{K}(4-5 \mathrm{MPa} \sqrt{\mathrm{m}})$, cross slip is hindered due to the small number of active slip systems, leading to the localization of slip on a limited number of slip systems and particle shearing, resulting in cracking occurring along planar slip bands [72].

For decreasing $\Delta \mathrm{K}$ testing at low $\mathrm{P}_{\mathrm{H} 2 \mathrm{O}}$ exposures, the low $\Delta \mathrm{K}$ behavior (which exhibited a flat non-cyrstallographic morphology) may be influenced by the interaction of the molecular transport with the crack wake roughness, so a low exposure $(0.5 \mathrm{~Pa})$ at constant low $\Delta \mathrm{K}(3 \mathrm{MPa} \sqrt{\mathrm{m}})$ test was performed to investigate the morphology of the cracking in the $\Delta \mathrm{K}$ regime below the UHV threshold without prior load effects. The results in Figure 18 show that in this low $\Delta \mathrm{K}$ and exposure regime, the cracking morphology is flat and non-crystallographic, despite the fact that SBC will occur at higher $\Delta \mathrm{K}$ values, as observed in Figures $7 \mathrm{c}$ and 9 . At $\Delta \mathrm{K}=3 \mathrm{MPa} \sqrt{\mathrm{m}}$ with no prior load effects, $\mathrm{SBC}$ is limited at low $\Delta \mathrm{K}$ for low $\mathrm{P}_{\mathrm{H} 2 \mathrm{O}}$ exposures. There remains a need to investigate if $\mathrm{SBC}$ could initiate at this low $\Delta \mathrm{K}$ if crack wake roughness was inputted into the surface initially which could lead to further insights into the influence of crack wake roughness on turbulent mixing, however this work is outside the scope of the current study.

These finding suggests either that at the lower $\Delta \mathrm{K}$ values: (1) there is a mechanically based change in the mechanism from cracking along the slip bands to non-crystallographic transgranular cracking, or (2) the crack growth rate is discontinuous thus altering the governing exposure parameter. Discontinuous crack growth occurs when an increasing number of cycles $(\Delta N)$ are 
needed between each crack advance. As the number of cycles increases, the time available for water vapor molecules to diffuse over the crack path increases. The discontinuous crack growth alters the effective frequency to $f / \Delta \mathrm{N}$, which drives the effective exposure parameter from $\mathrm{P}_{\mathrm{H} 20} / f$ to $\mathrm{P}_{\mathrm{H} 20} /(f / \Delta \mathrm{N})$; corresponding to a higher effective exposure that is potentially outside the molecular flow rate limited regime of the H-embrittlement process [1, 38, 73-74]. If discontinuous crack growth was occurring at these low $\Delta \mathrm{K}$, da/dN vs $\Delta \mathrm{K}$ behavior would be nearly independent of the exposure parameter $[1,38,73-74]$. When considering the post minima regime of the TTR, these results show that low $\Delta \mathrm{K}$ slip behavior and discontinuous crack growth behavior could be contributing to the mechanisms responsible for the increase in growth rates. The third hypothesis suggesting that elevated growth rates are a result of the continued strong contribution of turbulent mixing maintaining higher $\mathrm{P}_{\mathrm{H} 2 \mathrm{O}}$ will be addressed next.

\subsubsection{Crack Wake Induced Turbulent Mixing}

The Burns et al. paper proposed that after a certain critical level of roughness is reached in the crack wake, the $\mathrm{P}_{\mathrm{H} 20}$ transport mechanism changes to convective turbulent mixing as a result of crack wake asperity contact [1]. The convective mixing increases the supply of $\mathrm{P}_{\mathrm{H} 20}$ to the crack tip which helps to reignite the hydrogen environment embrittlement process and results in the subsequent rise in da/dN rates. Specifically, it is critical to establish the extent to which asperity contact is possible as the crack tip progresses and the SBC-based region of roughness is moved further behind in the crack wake. In order to do so, it is necessary to quantify how the crack wake opening profile evolves for the specific loading protocol used in the decreasing $\Delta \mathrm{K}$ experiments.

\subsubsection{Crack Opening Displacement Calculations}

Crack-opening displacement along the entire crack wake was calculated using a combination of methods in accordance with the work of Newman and Saxena [75-76]. Newman 
utilized a boundary collocation method to calculate the theoretical opening displacement at positions along the crack wake. The boundary collocation method incorporated an analytical solution that used boundary conditions where the complex-series stress functions were satisfied exactly on the crack surfaces [75]. Newman developed polynomial functions of crack lengthwidth ratio to calculate the crack opening displacement at five locations along the crack profile starting at $12.7 \mathrm{~mm}$ (corresponding to the location $\mathrm{V}_{2}$ in Figure 36a) to the right of the load line and ending at the crack mouth opening $(12.7 \mathrm{~mm}$ to the left of the load line and corresponding to $\mathrm{V}_{0}$ in Figure 36a) [75]. Newman represented the crack face profile from the crack tip to the load line as a straight line and did not incorporate crack tip opening displacement into the overall COD calculation (Figure 36a). Saxena expanded on Newman's method by incorporating an "axis of rotation" in the specimen crack profile to more accurately describe the elastic behavior of crack growth near the crack tip (Figure 36b) [76]. The axis of rotation factors into consideration crack tip constraint and instead uses a larger fictitious crack length known as a calculated by:

$$
\mathrm{a}_{0} / \mathrm{W}=0.0924 /\left(1-\mathrm{V}_{1} / \mathrm{V}_{0}\right)-0.25
$$

where $\mathrm{W}$ is the specimen width, $\mathrm{V}_{1}$ and $\mathrm{V}_{0}$ are the crack opening displacements at two locations along the crack wake profile, shown in Figure 36b, calculated using Newman's crack length polynomial functions. The red line in Figure $36 \mathrm{~b}$ represents the crack face profile for a crack of length $a_{0}$, compared to the crack profile in black which represents the crack face corresponding to the standard compliance calculated crack length.

For this work, the Newman/Saxena method was combined with elastic-plastic theory to accurately model the total crack opening displacement at a specific crack length. Figure 36c provides a crack profile schematic of the combined model that calculates crack mouth opening 
displacement (CMOD) using elastic theory and linear modeling of the crack surface. The angle of displacement ( $\theta$ in Figure 33c) was calculated by utilizing the equation below:

$$
\theta=\tan ^{-1}\left(0.5 \mathrm{CMOD} / \mathrm{a}_{\mathrm{o}}\right)
$$

where CMOD is the crack mouth opening displacement as calculated from the experimentally measured compliance data. By calculating the angle of displacement for any given loading condition, the crack opening displacement at any specific location of interest along the crack wake (labeled as " $\mathrm{x}$ " in Figure 36c) can be identified. This combined method makes it possible to graph the full crack wake profile for each crack length, based on the exact loading protocol used during testing. The total crack opening displacement at a specific location of interest $(\mathrm{x})$ was calculated using equation below:

$$
\operatorname{COD}_{\text {TOTAL } x}=2\left(a_{o}-x\right) * \tan (\theta)+\text { CTOD }
$$

where $\mathrm{x}$ is the crack length of interest, $\mathrm{a}_{0}$ is the fictious crack length factoring in the axis of rotation described above, $\theta$ is the angle of displacement between the crack surface and the crack tip opening displacement. The crack tip opening displacement (CTOD) is calculated using [77]:

$$
\mathrm{CTOD}=0.6 * \mathrm{~K}_{\max }{ }^{2} /\left(2 \sigma_{\mathrm{ys}} * \mathrm{E}\right)
$$

where $K_{\max }$ is the maximum stress intensity factor, $\mathrm{E}$ is Young's modulus, and $\sigma_{\mathrm{ys}}$ is the yield stress.

Crack-opening displacement calculations for the 1.8, 0.5, and 0.2 Pa exposures, tested under K-shed loading, were completed at locations corresponding to the start and end of the roughness span to investigate the possible effect the roughness magnitude could have on maintaining asperity contact. Each specimen exhibited a different crack length span of roughness corresponding to the threshold transition regime (listed in Table 5) and the roughness span 
increased with decreasing $\mathrm{P}_{\mathrm{H} 2 \mathrm{O}}$. Figure 37 provides a schematic of a $\mathrm{C}(\mathrm{T})$ fatigue crack growing with increased crack wake roughness. As the crack continues to grow, the COD calculations reflect the crack-opening displacement at the location of roughness indicated by the red line. Only the COD locations corresponding to the start of the span of roughness have been plotted in Figures 39,41 , and 43 . The black arrows in the optical fractographs serve to visually highlight the location corresponding to the COD calculations.

The crack-opening displacement at the location corresponding to the start of increased roughness $(\mathrm{a}=27.5 \mathrm{~mm})$ for the $1.8 \mathrm{~Pa}$ specimen was plotted as a function of crack length and shown in Figure 39. While the $\mathrm{x}$-axis plots increasing crack length, the y-axis is plotting the increasing crack-opening displacement at the location of $\mathrm{a}=27.5 \mathrm{~mm}$. The crack opening at a crack length of $27.5 \mathrm{~mm}$ steadily increased for maximum and minimum loading as the crack grew to a length of $42 \mathrm{~mm}$. Similar COD calculations were completed for the location of a $=30.0 \mathrm{~mm}$, corresponding to the end of any SBC or rougher portions of cracking. The crack-opening displacement calculations for the $0.5 \mathrm{~Pa}$ exposure were completed at crack lengths $\mathrm{a}=21.9$ and 25 $\mathrm{mm}$, with the COD results from $\mathrm{a}=21.9$ being plotted in Figure 41 . The crack-opening displacement calculations for the $0.2 \mathrm{~Pa}$ exposure were completed at crack lengths a=29.6 and 34.7 $\mathrm{mm}$, with the COD results from a=29.6 being plotted in Figure 43. Both exposures experienced increasing crack-opening displacement for maximum and minimum loading with growing crack length, but each profile had roughness spans that occurred at different crack lengths. As such, these COD values are specific for each exposure and the corresponding specific decreasing $\mathrm{K}$ loading protocol. Experimentally measured CMOD compliance data helped to ensure that a specific crack length was associated with the correct crack wake profile and crack-opening 
displacement. These specific locations were selected to enable direct comparison between observed roughness values in the crack wake and the crack opening at that location.

\subsubsection{Roughness Characterization in the Threshold Transition Regime}

In order to fully characterize the potential for crack wake asperity contact to occur, an accurate representation of the localized roughness for each fracture surface in the TTR was needed. Figures 19a, 20a, and 21a show the 3-D characterization of the fracture surface and the corresponding optical fractograph for the 1.8, 0.5, and 0.2 Pa samples, respectively. The average $\mathrm{Ra}$ and $\mathrm{Rq}$ values for the three exposures $(0.2,0.5$, and $1.8 \mathrm{~Pa})$ with a TTR regime and two higher humidity exposures (4 and $2668 \mathrm{~Pa}$ ) are presented in Tables 1-3. Table 1 shows that crack face topography roughness increased with decreasing $\mathrm{P}_{\mathrm{H} 2 \mathrm{O}}$. Additionally, the span of this localized roughness increased with decreasing $\mathrm{P}_{\mathrm{H} 2 \mathrm{O}}$ as shown in Table 5. After the threshold transition regime, the surface roughness of the $0.2,0.5$, and $1.8 \mathrm{~Pa}$ exposures decreased to values similar to the average roughness measured for the 4 and $2668 \mathrm{~Pa}$ exposures (Tables 2 and 3). These findings support the optical fractograph conclusions that the magnitude of SBC and faceted features increased with decreasing $\mathrm{P}_{\mathrm{H} 2 \mathrm{O}}$ [1]. Additionally, the local crack face topography was examined to determine the average height of crack asperities. The average Rp and Rv values increased with decreasing $\mathrm{P}_{\mathrm{H} 2 \mathrm{O}}$, which agreed well with the previous roughness measurements above. To summarize, the localized roughness area associated with the TTR increased in magnitude and span, as well as average asperity height, with decreasing $\mathrm{P}_{\mathrm{H} 2 \mathrm{O}}$

In comparing the COD to the measured asperity metrics, it is critical to recognize that the degree of contact will depend on both the measured topography and the Mode II displacement that will offset the features on either side of the crack wake and cause contact [78]. To compare the 
surface roughness characterization and the crack-opening displacement calculations, three different Mode II displacement conditions were considered. Figure 38 shows example crack asperity schematics for the upper and lower bounds considered (Figure 38a and 38c), as well as a condition that would be representative of the middle (Figure 38b). First, Figure 38a illustrates a condition where the Mode II displacement is equal to the CTOD, representing the minimum or lower bound of Mode II displacement that would cause asperity contact. Figure 38c illustrates a condition where the Mode II displacement results in opposing crack asperities that are directly aligned, resulting in peaks and valleys directly opening and closing on top of each other. This condition would represent the maximum or upper bound of Mode II displacement that would cause direct asperity contact. The magnitude of the total crack asperity height (Rpv) was used to quantify this displacement condition with the localized roughness. Second, Figure $38 \mathrm{~b}$ illustrates a condition where the amount of Mode II displacement results in $80 \%$ of the crack asperity height remaining in contact with the opposing face during loading. This situation was quantified as having a crack asperity height magnitude of $20 \%$ of the total crack asperity height $(0.2 * \mathrm{Rpv})$.

The final condition was selected based on the findings of Suresh and Ritchie that suggested that the Mode II displacement may be as much as $30 \%$ of the Mode I displacement at nearthreshold stress intensities for 1018 steel with no environmental effects [78]. This study also established a model for predicting crack closure induced by surface roughness which established that crack wake roughness and some degree of Mode II displacement are required for asperity contact to occur. It is acknowledged that extrapolating the Mode II/Mode I $=0.3$ relationship to a different material and environment may not provide the most accurate representation of Mode II displacement in 7075-T651, but it will enable a comparison to build on. A reasonable measure of the Mode I displacement at the crack tip given by the CTOD value which is estimated as [77]: 


$$
\mathrm{CTOD}=0.6 * \mathrm{~K}_{\max }^{2} /\left(2 \sigma_{\mathrm{ys}} * \mathrm{E}\right)
$$

where $K_{\max }$ is the maximum stress intensity factor, $\mathrm{E}$ is Young's modulus, and $\sigma_{\mathrm{ys}}$ is the yield

stress. The corresponding Mode II displacement was calculated by multiplying the CTOD by 0.3.

At the location of highest roughness, the maximum CTOD was used to calculate the Mode II displacement. This value (shown in Table 6) was then divided by Rpv to allow for direct comparisons to be made within the conditions. The CTOD values and Rpv values were different for each exposure which results in each condition having a different percentage of Rpv to signify when the Mode II/Mode I displacement ratio equaled 0.3. Table 7 summarizes the three different Mode II displacement conditions ( Rpv, 0.2*Rpv, Mode II/Mode I = 0.3) and the corresponding magnitudes in meters for each of the conditions that were used in comparison with the COD. Figures 39, 41, and 43 plot the respective magnitude of the two middle conditions for Mode II displacement (listed in Table 7) in addition to the COD at the start of the roughness for each of the three exposures.

\subsubsection{Comparison of the Crack Wake Roughness Topography and the Crack Opening Displacement}

In order to quantitatively evaluate the degree of asperity contact in the crack wake, as pertinent to the proposed turbulent mixing hypothesis, crack opening displacement measurements were coupled with characterization of the fracture surface roughness in order to better understand the extent of crack wake asperity contact occurring in the post-minima regime.

A metric to determine the presence of crack wake asperity contact during the threshold transition regime was illustrated by plotting the effective crack opening $\left(\delta_{\text {eff }}\right)$ over the nominal crack opening $\left(\delta_{\text {nom }}=\mathrm{COD}\right.$ at the maximum load $-\mathrm{COD}$ at the minimum load $)$. These values were calculated at 
two fixed positions in the crack wake, corresponding to the start and end of the roughness, for values of increasing crack length and measured stresses observed during the decreasing $\Delta \mathrm{K}$ protocol. The effective crack opening was defined as the difference between the maximum COD occurring during the loading cycle and asperity contact height calculated for each of the Mode II displacement conditions listed in Table 7. According to this metric, crack wake asperity contact is occurring when $\delta_{\text {eff }} / \delta_{\text {nom }}<1$. When Rpv is larger than the maximum opening at that crack length, $\delta_{\text {eff }} / \delta_{\text {nom }}$ is a negative number indicating asperity contact is occurring over the entire loading cycle; in theory the more negative the number the more load transfer or closure would be occurring. When $0<\delta_{\text {eff }} / \delta_{\text {nom }}<1$, crack wake asperity contact is occurring over a portion of the loading cycle. When $\delta_{\text {eff }} / \delta_{\text {nom }}>1$, crack wake asperity contact at the positions of interest in the crack wake is not occurring. According to the proposed hypothesis when $\delta_{\text {eff }} / \delta_{\text {nom }}>1$, the molecular transport would not be influenced by turbulent mixing and would revert to being controlled by diffusion based (Knudsen flow) or advection-based molecular transport. The $\delta_{\text {eff }} / \delta_{\text {nom }}$ metric enables quantification of the degree of asperity contact that can be expected in the rough region as the crack continues to grow; evaluating this metric for large ( $R p v$ and $0.2 \mathrm{Rpv}$ ) and small (0.00256*Rpv for $0.2 \mathrm{~Pa}, 0.00375^{*} \mathrm{Rpv}$ for $0.5 \mathrm{~Pa}$, and $0.00689^{*} \mathrm{Rpv}$ for $1.8 \mathrm{~Pa}$ ) Mode II displacements will provide upper and lower bound estimates of the degree of contact. Additionally, the span over which asperity contact is acting, indicated by looking at $\delta_{\text {eff }} / \delta_{\text {nom }}$ at both the beginning and end of the roughness region, is identified for each loading protocol and environment of interest.

Figures 40,42 , and 44 show the relationship between $\delta_{\text {eff }} / \delta_{\text {nom, }}$ calculated at both the beginning and the end of the roughness span (measured from Figures 19-21) plotted versus the location of the growing crack tip for the 1.8, 0.5, and $0.2 \mathrm{~Pa}$ exposures, respectively. When 
comparing the magnitudes of the three Mode II displacement conditions to the COD for each exposure, the upper bound (Rpv in Figure 38c) was 3-5 times larger than the crack opening displacement which would indicate that high levels of crack wake asperity contact are occurring for the duration of the TTR. The changing trend in the da/dN behavior does not support this high level of asperity contact, so work focused on characterizing the $\delta_{\text {eff }} / \delta_{\text {nom }}$ behavior for the $0.2 * \operatorname{Rpv}$ (Figure 38b) and the Mode II/Mode I =0.3 lower bound conditions (Ritchie and Suresh).

The $\delta_{\text {eff }} / \delta_{\text {nom }}$ behavior for the $1.8 \mathrm{~Pa}$ exposure plotted for the $0.00689 * \mathrm{Rpv}$ Mode II displacement condition showed values greater than 1 for the entire duration of the TTR for both the start and end of the roughness, suggesting that crack wake asperity contact was not taking place under these conditions (Figure 39). The $\delta_{\text {eff }} / \delta_{\text {nom }}$ behavior plotted for the $0.2 * R p v$ Mode II displacement condition showed that, at the location near the start of the roughness $(\mathrm{a}=27.5 \mathrm{~mm})$, $\delta_{\text {eff }} / \delta_{\text {nom }}$ was between 0 and 1 for crack lengths $\mathrm{a}=27.5-31 \mathrm{~mm}$, indicating that some degree of crack wake asperity contact was occurring over a portion of the loading cycle at this location for $3.5 \mathrm{~mm}$ of crack growth. At a roughness location of a= $30 \mathrm{~mm}$ (the end of the roughness span), $\delta_{\text {eff }} / \delta_{\text {nom }}$ was between 0 and 1 for crack lengths $\mathrm{a}=30-38 \mathrm{~mm}$. The combination of the results from these two locations show that for the $0.2 * \mathrm{Rpv}$ Mode II displacement condition, partial asperity contact is occurring for $10.5 \mathrm{~mm}$ of crack growth $(\mathrm{a}=27.5-38 \mathrm{~mm})$ for $\mathrm{P}_{\mathrm{H} 2 \mathrm{O}}=1.8 \mathrm{~Pa}$ and $\Delta \mathrm{K}$-shed loading.

The $\delta_{\text {eff }} / \delta_{\text {nom }}$ behavior for the $0.5 \mathrm{~Pa}$ exposure plotted for the $0.00375 * \mathrm{Rpv}$ Mode II displacement condition also showed values greater than 1 for the entire duration of the TTR for both the start and end of the roughness, suggesting that crack wake asperity contact was not taking place under these conditions (Figure 42). The $\delta_{\text {eff }} / \delta_{\text {nom }}$ behavior for the $0.2 *$ Rpv Mode II displacement condition showed that at the location near the start of the roughness $(\mathrm{a}=21.9 \mathrm{~mm})$, 
$\delta_{\text {eff }} / \delta_{\text {nom }}$ was between 0 and 1 for crack lengths $\mathrm{a}=21.9-30 \mathrm{~mm}$, indicating that some degree of partial crack wake asperity contact was occurring for $3.5 \mathrm{~mm}$ of crack growth. At a roughness location of $\mathrm{a}=25 \mathrm{~mm}$ (the end of the roughness span), $\delta_{\text {eff }} / \delta_{\text {nom }}$ was less than zero for crack lengths $\mathrm{a}=25-29 \mathrm{~mm}$ followed by $\delta_{\text {eff }} / \delta_{\text {nom }}$ behavior between 0 and 1 for crack lengths a $=29-33 \mathrm{~mm}$ (the end of the K-shed loading segment). This plot suggests that at the location $\mathrm{a}=25 \mathrm{~mm}$, asperity contact was occurring over the entire loading cycle between a $=25-27 \mathrm{~mm}$ and transitioned to partial asperity contact for the duration of the testing segment. The combination of the results from these two locations show that for the $0.2 * \mathrm{Rpv}$ Mode II displacement condition, $11.5 \mathrm{~mm}$ of crack growth is experiencing some form of partial asperity contact during crack growth from $\mathrm{a}=$ 21.9 - $34 \mathrm{~mm}$.

The $\delta_{\text {eff }} / \delta_{\text {nom }}$ behavior for the $0.2 \mathrm{~Pa}$ exposure plotted for the $0.00259 * \mathrm{Rpv}$ Mode II displacement condition also showed values greater than 1 for the entire duration of the TTR for both the start and end of the roughness, suggesting that crack wake asperity contact was not taking place under these conditions (Figure 44). The $\delta_{\text {eff }} / \delta_{\text {nom }}$ behavior for the $0.2 *$ Rpv Mode II displacement condition showed that at the location near the start of the roughness $(\mathrm{a}=29.6 \mathrm{~mm})$, $\delta_{\text {eff }} / \delta_{\text {nom }}$ was below zero for crack lengths a $=29.6-36 \mathrm{~mm}$ followed by $\delta_{\text {eff }} / \delta_{\text {nom }}$ behavior between 0 and 1 for crack lengths $\mathrm{a}=36-38 \mathrm{~mm}$ (the end of the $\mathrm{K}$-shed loading segment). At a crack surface location of $\mathrm{a}=34.7 \mathrm{~mm}$ (the end of the roughness span), $\delta_{\text {eff }} / \delta_{\text {nom }}$ was less than zero for the entire duration of crack growth from $\mathrm{a}=34.7-38 \mathrm{~mm}$. This plot suggests that the location corresponding to the start of the roughness experienced asperity contact occurring over the entire loading cycle between crack growth from $\mathrm{a}=29.6-36 \mathrm{~mm}$ before transitioning to partial contact from $36 \mathrm{~mm}$ to $38 \mathrm{~mm}$. The crack location at the end of the roughness ( $\mathrm{a}=34.7 \mathrm{~mm}$ ) experienced asperity contact during the full load cycle from crack growth from $34.7-38 \mathrm{~mm}$. The combination 
of the results from these two locations show that for the $0.2 * \mathrm{Rpv}$ Mode II displacement condition, $8.4 \mathrm{~mm}$ of crack growth is experiencing some form of asperity contact starting with full asperity contact occurring from $\mathrm{a}=29.6-36$ transitioning to partial asperity contact occurring for $36-38$ mm.

When comparing the crack wake asperity contact behavior for each exposure, the Suresh and Ritchie proposed amount of Mode II displacement yielded no crack wake asperity contact. According to the proposed hypothesis [1], molecular transport would continue to be controlled by diffusion based (Knudsen flow) or advection-based molecular transport without asperity contact. These results would not explain the upturn in da/dN rates. When using a higher amount of Mode II displacement $(0.2 * \mathrm{Rpv})$, each exposure exhibited varying degrees of crack wake asperity contact. This analysis is complicated by the uncertainty associated with the value of Mode II displacement to investigate; however, further analysis in this study uses the results for the $0.2 * \mathrm{Rpv}$ displacement condition to represent a reasonable Mode II displacement to show the crack wake asperity contact behavior during the TTR.

The span of affected crack growth for the three exposures is difficult to compare, because each exposure ended the $\mathrm{K}$-shed loading protocol at different $\Delta \mathrm{K}$ values. While a direct comparison cannot be made between the duration of affected crack growth, the extent (full vs. partial) of crack wake asperity contact can be considered across the three exposures. The $0.2 \mathrm{~Pa}$ exposure was the only sample to show a high degree of asperity contact during the entire load cycle throughout the TTR $(6.4 \mathrm{~mm}$ of partial contact and $2 \mathrm{~mm}$ of full contact). The $0.5 \mathrm{~Pa}$ exposure experienced $12.1 \mathrm{~mm}$ of crack growth with $4 \mathrm{~mm}$ of full asperity contact and $8.1 \mathrm{~mm}$ of partial asperity contact. The $1.8 \mathrm{~Pa}$ exposure experienced $10.5 \mathrm{~mm}$ of crack growth corresponding with partial asperity contact occurring during loading. As $\mathrm{P}_{\mathrm{H} 2 \mathrm{O}}$ increased, the severity of the crack 
wake asperity contact (full vs. partial contact) decreased presumably due to a reduced fraction of SBC facets.

For the $0.5 \mathrm{~Pa}$ sample, the crack front experienced partial crack wake contact transitioning to full asperity contact for the crack lengths $\mathrm{a}=21.9-25 \mathrm{~mm}$. This crack span correlates to the roughness span and the drop and upturn in $\mathrm{da} / \mathrm{dN}$ rates for the TTR. The $0.5 \mathrm{~Pa}$ crack front then experienced full asperity contact during the total load cycle $(\mathrm{a}=25-29 \mathrm{~mm})$ before transitioning to partial crack wake asperity contact and ultimately unimpeded flow. The spike in da/dN (Figure 12) occurred at a crack length $(\mathrm{a}=25.6 \mathrm{~mm})$ just following this period of increased contact asperity contact. For the $0.2 \mathrm{~Pa}$ sample, the crack front experienced full crack wake asperity contact for the entire duration of the TTR corresponding to $\mathrm{a}=29.6-38 \mathrm{~mm}$. Figure 13 showed that the spike in da/dN rates occurred at a crack length $\mathrm{a}=34.4 \mathrm{~mm}$, corresponding to this region of full asperity contact occurring. If the Mode II displacement value is reasonable, then this correlation between the degree of asperity contact and the up-turn would support the proposed turbulent mixing hypothesis.

The ACR and ASTM 2\% ratio were plotted for each exposure to investigate whether strong crack closure could affect da/dN behavior during the TTR. The 1.8 Pa exposure started to exhibit the effects of crack closure at $\mathrm{a}=33 \mathrm{~mm}(5.5 \mathrm{~mm}$ after the start of the SBC cracking) with a $5 \%$ reduction in the $\Delta \mathrm{K}_{\text {eff }}$ increasing to a $15 \%$ reduction for the ACR and a $20 \%$ reduction in the $\Delta \mathrm{K}_{\text {eff }}$ increasing to a $30 \%$ reduction for the ASTM $2 \%$ at the end of the K-shed loading protocol $(\mathrm{a}=43$ mm) (Figure 39). The 0.5 Pa exposure started to exhibit the effects of crack closure starting at a $=25 \mathrm{~mm}(3.1 \mathrm{~mm}$ after the start of the $\mathrm{SBC}$ cracking $)$ with a $5 \%$ reduction in the $\Delta \mathrm{K}_{\mathrm{eff}}$ increasing to a $15 \%$ reduction for the ACR and a $20 \%$ reduction in the $\Delta \mathrm{K}_{\text {eff }}$ increasing to a $45 \%$ reduction for the ASTM 2\% at the conclusion of the K-shed loading protocol $(\mathrm{a}=34 \mathrm{~mm})$ (Figure 41). The 
0.2 Pa exposure started to exhibit the effects of crack closure starting at the start of the roughness $\left(\mathrm{a}=29.6 \mathrm{~mm}\right.$ ) with a $5 \%$ reduction in the $\Delta \mathrm{K}_{\text {eff }}$ increasing to a $30 \%$ reduction for the ACR and a $30 \%$ reduction in the $\Delta \mathrm{K}_{\text {eff }}$ increasing to a $65 \%$ reduction for the ASTM $2 \%$ at the conclusion of the K-shed loading protocol $(\mathrm{a}=34 \mathrm{~mm})$ (Figure 43). These plots indicate that closure is playing an increasing role during the threshold transition progression, but the $\mathrm{da} / \mathrm{dN}$ behavior does not scale with the rise in $\mathrm{da} / \mathrm{dN}$ suggesting that crack closure is not the controlling mechanism for the increasing portion of the TTR. As the observed roughness increased with decreasing $\mathrm{P}_{\mathrm{H} 2 \mathrm{O}}$, the amount of roughness induced crack tip closure increased as well. Roughness induced closure could serve as a possible indicator of the presence of crack wake asperity contact. For the 0.2 and 0.5 $\mathrm{Pa}$ exposures, the points where crack closure effects are detected $(\mathrm{a}=29.6$ and $25 \mathrm{~mm}$, respectively) for the ASTM 2\% ratio correspond directly to the beginning of full crack wake asperity contact experienced by both maximum and minimum loading. The closure data presented in Figures 39c, 41c, and 43c were used to make an informed selection for the Mode II displacement condition that would be modeled. The $0.2 \mathrm{Rpv}$ condition (Figure $38 \mathrm{~b}$ ) calibrated the $\delta_{\text {eff }} / \delta_{\text {nom }}$ behavior to agree with observed closure trends. All three exposures showed increasing effects of crack closure as the crack progressed which could indicate lingering effects of crack asperity contact or simply be a function of the longer crack, lower loads, thus decreased crack tip angle (Figures 39c, 41c, and 43c).

Comparing the crack wake asperity contact behavior for each exposure indicates that there is some degree of crack wake asperity contact occurring from the start of the roughness for all three exposures. The molecular transport hypothesis argued that once a sufficient degree of crack wake asperity contact started to occur, turbulent mixing would increase the crack tip $\mathrm{P}_{\mathrm{H} 2 \mathrm{O}}$ and $\mathrm{da} / \mathrm{dN}$ would begin to rise. The observed crack wake asperity contact behavior for each exposure 
would imply that there is crack wake asperity contact occurring during the impeded crack growth segment, instead of relying on a certain amount of roughness being achieved. These results indicate that the interaction between the surface roughness, crack wake asperity contact, and turbulent mixing may be more intricately connected then simply the onset of first the asperity contact. The role crack wake asperity contact plays in controlling the upturn in $\mathrm{da} / \mathrm{dN}$ rates cannot be fully determined until the Mode II displacement occurring is accurately measured.

The correlation between when both the start and end of roughness experienced $\delta_{\text {eff }} / \delta_{\text {nom }}$ values greater than zero was examined for the three exposures. This point would represent when the localized roughness is not larger than the magnitude of the crack opening (i.e. there is no asperity contact at $\mathrm{K}_{\max }$ ), so water molecules would be able to travel into the crack channel to the crack tip. Both the 0.5 and $1.8 \mathrm{~Pa}$ exposures had $\delta_{\text {eff }} / \delta_{\text {nom }}$ values that transitioned to being greater than zero at a crack length that corresponded to the da/dN behavior at the start of the systematically decreasing da/dN portion that mirrored higher exposures after the TTR (Figure 12 - dotted line for $0.5 \mathrm{~Pa}) . \delta_{\text {eff }} / \delta_{\text {nom }}$ values were greater than zero starting at a crack length of a $=31.5 \mathrm{~mm}$ for the 1.8 $\mathrm{Pa}$ exposure and $\mathrm{a}=28.5 \mathrm{~mm}$ for the $0.5 \mathrm{~Pa}$ exposure. Both 0.5 and $1.8 \mathrm{~Pa}$ began to resume expected da/dN vs $\Delta \mathrm{K}$ trends as the channel became open $\left(\delta_{\text {eff }} / \delta_{\text {nom }}>0\right)$ to allow the flow of water vapor molecules. These results suggest that a certain amount of channel opening is needed to allow molecules to travel before the presence of asperity contact can help to impart momentum to the water vapor molecules. The chosen contact metric indicates the presence of crack wake asperity contact, but the role it plays in controlling the upturn in $\mathrm{da} / \mathrm{dN}$ rates and the potential effect of molecular transport from the sides is not sufficiently understood. These results do suggest that asperity contact will be maintained even as the crack progresses $10-15 \mathrm{~mm}$ beyond the roughness, 
but the degree to which this contact will influence the molecular flow behavior to the crack tip is still in question.

As established with the crack wake roughness experiments and marker-band experiments, the molecular flow from the sides is critical and could play a role in controlling the rise in $\mathrm{da} / \mathrm{dN}$ out of the TTR. It appears that a certain amount of crack extension inwardly from the sides is also required in order to interact with the roughness at the center of the sample and initiate turbulent mixing. Results suggest that the upturn in $\mathrm{da} / \mathrm{dN}$ is more complicated than initial molecular transport hypothesis [1]. Testing indicates that the upturn in $\mathrm{da} / \mathrm{dN}$ is driven by the interaction of molecular transport, closure, asperity based mixing, and position dependent $\Delta \mathrm{K}$. The fluctuations in the da/dN behavior of the $3 \mathrm{~mm}$ crack wake roughness experiment showed a similar transition from impeded to enhanced crack growth rates that was first observed for the post-minima regime in the K-shed loading protocol. The possible correlation between the surface roughness and the enhanced growth rates could help in identifying the controlling mechanisms in the post-minima regime for the K-shed loading experiments.

\subsubsection{Influence of crack wake roughness $10-15 \mathrm{~mm}$ behind the crack tip on molecular transport}

Insights into the degree to which crack wake roughness that is $10-15 \mathrm{~mm}$ behind the crack tip influences the molecular flow can be considered by analyzing the two 7075-T651 samples with different magnitudes of crack wake roughness. These growth rate data showed that as the crack continued to grow, the da/dN behavior began to fluctuate; directly corresponding to changes in the fracture surface morphology (Figures 14 and 16). Both samples exhibited da/dN behavior that began to fluctuate after the initial $4 \mathrm{~mm}$ of crack growth. The $3 \mathrm{~mm}$ experiment showed 5 cycles 
of da/dN decreasing for $1 \mathrm{~mm}$ before increasing for another $1 \mathrm{~mm}$ to reach a similar maximum da/dN (Figure 16). This self-perpetuating cycle showed near perfect repeatability four times during the course of testing while $\mathrm{P}_{\mathrm{H} 20} / f$ and $\Delta \mathrm{K}$ were held constant. The fluctuations seen for the $0.5 \mathrm{~mm}$ span of roughness were not repeatable in shape or occurrence (Figure 14). The results of these varying roughness span experiments will be addressed in regards to investigating: (1) the difference in growth rate data and corresponding fracture surface between the 0.5 and $3 \mathrm{~mm}$ samples and (2) the fluctuations in the context of the crack wake asperity contact hypothesis presented above.

The reason for the difference in the da/dN fluctuations between the two experiments is unclear and it is difficult to tell if this deviation is systematic and repeatable or if this is just experimental variation. The repeatability of the fluctuations within the $3 \mathrm{~mm}$ experiment would support the idea that this is a systematic and repeatable effect of roughness on $\mathrm{da} / \mathrm{dN}$. If this behavior is real, the differences in the fluctuation behavior between the $0.5 \mathrm{~mm}$ and $3 \mathrm{~mm}$ data would suggest that the crack wake roughness continues to influence the molecular transport and crack growth behavior even after the crack grows well beyond the roughness. As described in Section 4.1.2, the initial impedance in growth rates for the $3 \mathrm{~mm}$ sample is suggested to be a result of an irregular crack front. The center of the specimen is initially impeded resulting in environmentally enhanced cracking along the sides. As the crack front at the center "catches up," the crack front becomes more regular in shape and the center is once again impeded resulting in the initiation of SBC-like features (Figure 17.D2); resulting in a repetition of the crack front progression sequence shown in Figure 34. This cycle was repeated four times in sixteen millimeters of crack growth (Figure 16). The irregularity in the fluctuations in the $0.5 \mathrm{~mm}$ sample could be explained by diffusion distances (notch or inwardly from the sides) competing for 
dominance resulting in unsystematic oscillations in da/dN. Completing additional experiments with this same subcritical roughness and other additional magnitudes of SBC would help to further investigate the deviation in da/dN vs crack length, however this work is outside the scope of the current study

Next, the roughness experiments were examined to see if the fluctuations in $\mathrm{da} / \mathrm{dN}$ and the fracture surface morphology could be explained in relation to the crack wake asperity contact hypothesis presented above. Both the $0.5 \mathrm{~mm}$ and $3 \mathrm{~mm}$ sample showed $2 \mathrm{~mm}$ of crack extension from the sides following different initial area sizes of SBC that covered the entire thickness of the specimen. It appears a certain amount of crack extension inwardly $(1-2 \mathrm{~mm})$ from the sides as well as the presence of crack wake asperity contact is required in order to initiate turbulent mixing. This could support the claim that a certain amount of channel opening is needed for molecules to be able to travel to the crack tip before the presence of asperity contact can help to impart momentum. Further research would need to investigate and quantify the possible molecular transport effects of having crack wake asperity contact occurring only in the center of the specimen and cracking dominating in from the sides. These constant $\Delta \mathrm{K}, \mathrm{P}_{\mathrm{H} 20} / f$ experiments serve to show that the interaction between the roughness on the fracture surface and the molecular transport continues to affect crack growth rates as the crack wake roughness moves $10-15 \mathrm{~mm}$ behind the crack tip.

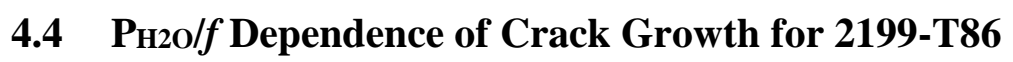

$\mathrm{Al}-\mathrm{Cu}-\mathrm{Li}$ alloys have shown superior fatigue performance when compared to $\mathrm{Al}-\mathrm{Zn}-\mathrm{Mg}-\mathrm{Cu}$ alloys for a wide range of environmental exposures [40-43]. Researchers have attributed this increased fatigue resistance due to the beneficial interaction of slip with microstructural features 
and the resulting influence on the crack tip driving force [25]. The superior fatigue resistance is controlled by two factors: (1) increased extrinsic shielding as a result of crack closure and (2) reduced environmental embrittlement when compared to 7075-T651 [44]. The primary strengthening phase in the $7 \mathrm{xxx}$-series alloy equilibrium is incoherent $\eta$-phase (or the semicoherent metastable $\eta^{\prime}$-phase $)\left(\mathrm{Mg}(\mathrm{Zn}, \mathrm{Al}, \mathrm{Mg})_{2}\right) \quad$ [25]. This non-shearable incoherent phase encourages dislocation looping and heterogenous slip. In Al-Cu-Li alloys, the shearable $\delta$ '-phase $\left(\mathrm{Al}_{3} \mathrm{Li}\right)$ enables homogenous reversible planar slip which serves as an extrinsic toughening mechanism that helps to reduce the crack tip driving force [40-44]. Peak aged Al-Li microstructures are hardened by the presence of ordered coherent precipitates that dictate the localized planar and reversible slip character of the alloy [40]. The Al-Li based alloys exhibit faceted cracking (SBC-like features) in inert environments, regardless of $\Delta \mathrm{K}$ and temper [80]; suggesting that 2199-T86 would also have a different environmental dependence at low $\Delta \mathrm{K}$ and low water vapor pressure than that seen for 7075-T651 [44, 80]. The different crack morphology in 2199-T86 is of particular importance to the potential of a similar threshold transition regime. The threshold transition regime for 7075-T651 is predicated on the fact that SBC-like features are promoted at low water vapor pressures/low temperatures at intermediate to low $\Delta \mathrm{K}$ values. The extent of this environmental threshold is also affected by the amount of roughness interacting with the molecular transport from the crack mouth to crack tip. As such, it would be expected that the 2199-T86 data would behave differently since faceted cracking can persist to higher $\Delta \mathrm{K}$ values.

Fatigue crack growth rates in 2199-T86 (L-T) were seen to decrease with decreasing water vapor pressure over a wide stress intensity range $(\Delta \mathrm{K})$ (Figure 22$)$. For $\mathrm{P}_{\mathrm{H} 2 \mathrm{O}}$ above $38 \mathrm{~Pa}, \mathrm{da} / \mathrm{dN}$ decreased with decreasing $\Delta \mathrm{K}$ in four power law segments consistent with the HEE process [1, 19-21]. The equivalence of the da/dN rates observed at $0.5 \mathrm{~Pa}$ and UHV in 2199-T86 illustrates 
the elimination of environmental influences at a higher exposure than 7075-T651. A rapid decrease in da/dN was observed for $\mathrm{P}_{\mathrm{H} 2 \mathrm{O}}$ values of $18,1.8$, and $4 \mathrm{~Pa}$ between a $\Delta \mathrm{K}$ of 8-7 MPa $\sqrt{\mathrm{m}}$. The da/dN minima occurred for a $\Delta \mathrm{K}$ range of 6.7 to $7.0 \mathrm{MPa} \sqrt{\mathrm{m}}$, before systematically increasing with decreasing $\Delta \mathrm{K}$. The threshold transition regime behavior for 2199-T86 occurred at a higher range of $\mathrm{P}_{\mathrm{H} 2 \mathrm{O}}$ and stress intensity range than 7075-T651. For all decreasing $\Delta \mathrm{K}$ experiments between $4 \mathrm{~Pa}$ and $0.5 \mathrm{~Pa}$ (Figure 26b-26d), the 2199-T86 fracture surfaces showed a transition from a smooth fracture surface to a rough surface in the middle. The change in morphology correlates with the threshold transition region observed for the 18, 1.8, and 4 Pa exposures. Unlike 7075-T651, the 2199-T86 fractographic surfaces showed SBC in inert environments for all $\Delta \mathrm{K}$ (Figure 28-32). At higher $\Delta \mathrm{K}$ for UHV (Figure 29a, 30a, and 31a), the fracture surface transitioned to $\mathrm{SBC}$, whereas 7075 showed flat void-like features at higher $\Delta \mathrm{K}$. The similarities in the scope and span of the roughness transition in 2199-T86 suggest a similar threshold transition regime that depends on $\Delta \mathrm{K}$ and the environment similar to the molecular transport based theory developed for 7075-T61 [1]

The da/dN vs $\Delta \mathrm{K}$ behavior of the 2199-T86 specimens with reduced thickness also supported the molecular transport hypothesis proposed for 7075-T651. By reducing the thickness, the molecular flow distance from the sides was decreased resulting in faster growth rates. The sidegrooved sample tested at UHV did not show a shift in the da/dN vs $\Delta \mathrm{K}$, showing that the thickness dependence of da/dN was only observed in the molecular flow controlled regime (Figure 24). Additionally, the threshold transition regime for the 1.8 and $4 \mathrm{~Pa}$ side-grooved tests was similar in magnitude and shape, but occurred at higher $\Delta \mathrm{K}$ when compared to the TTR behavior for the thicker specimens. The side-grooving process reduces the cross-sectional area for the Mode I crack and reduces the molecular flow distance for diffusion, resulting in faster growth rates at similar 
$\Delta \mathrm{K}$ values. The pertinent diffusion distance for molecular transport to the crack tip is the through thickness direction, as such, changing the specimen geometry reasonably influences the environmental crack behavior at intermediate exposures. This behavior supports that the molecular transport in the through thickness dimension controls the environmental influence, particularly in the TTR regime as previously seen with 7075-T651. The differences in crack morphology for 2199-T86 could result in a differing effect of surface roughness and crack wake asperity contact on da/dN behavior. It would be beneficial to complete similar COD vs asperity height analysis to determine if any more information can be gleaned on the post-minima regime.

\subsection{Impact on Fatigue Life Prediction}

The $7075-\mathrm{T} 651$ and $2199-\mathrm{T} 86 \mathrm{da} / \mathrm{dN}$ vs $\Delta \mathrm{K}$ data for varying $\mathrm{P}_{\mathrm{H} 2 \mathrm{O}}$ illustrated that there can be a significant reduction in crack growth rates in high altitude environments pertinent to airframe operations. The water vapor pressure over frequency $\left(\mathrm{P}_{\mathrm{H} 2 \mathrm{O}} / f\right)$ exposure parameter is used as a conservative proxy to model the environmental influence on fatigue cracking, but does not fully capture the effect of temperature on fatigue growth rates [25-26]. To that end, efforts are underway to investigate the beneficial effect of low temperature on the fatigue crack growth behavior beyond that associated with the reduction in the $\mathrm{P}_{\mathrm{H} 2 \mathrm{O}}$ [25]. Analysis detailed above indicates that the threshold transition behavior observed in 7075-T651 and 2199-T86 is governed by molecular transport that can be affected by the loading protocol, specimen geometry, and/or testing configuration. While the false threshold behavior resulting in the dip in $\mathrm{da} / \mathrm{dN}$ is real and repeatable, such behavior is geometry and molecular flow path dependent and should not be incorporated into fracture mechanics based predictions. This behavior at intermediate $\Delta \mathrm{K}$ and $\mathrm{P}_{\mathrm{H} 2 \mathrm{O}}$ will vary with crack geometry, loading ratio, $\Delta \mathrm{K}$, and environmental exposure and should 
not be incorporated in lifetime prognosis for real airframe structures. Several potential solutions are available. First, develop a protocol for crack growth rate generation that limits the threshold transition regime behavior. One example of such protocol could be to include a K-rise protocol that has a compression precrack, but the effect of the testing protocol on the $\mathrm{da} / \mathrm{dN}$ vs. $\Delta \mathrm{K}$ data is needed before incorporation. Second, use an interpolation from high $\Delta \mathrm{K}$ cracking to low $\Delta \mathrm{K}$ cracking that will capture the broad non-TTR influenced effect of the environment, but eliminate the TTR behavior. This method is currently being implemented in a companion study that uses these data to augment LEFM modeling tools (AFGROW) with the ability to incorporate environmental effects. The interpolated data provided to colleagues for this effort are included in Table 8 and Figure 45.

Failure to recognize the "apparent threshold" behavior could lead to highly non-conservative LEFM predictions if experimental tests are concluded in the threshold transition regime. A common stopping experimental cracking threshold is $1.0 \times 10^{-6} \mathrm{~mm} / \mathrm{cycle}$, which falls in the middle of the threshold transition regime for lower exposures. Concluding an experiment at this growth rate would imply that crack growth would not occur for $\Delta \mathrm{K}<4 \mathrm{MPa} \sqrt{\mathrm{m}}$. Testing to a crack growth threshold of $1.0 \times 10^{-7} \mathrm{~mm} /$ cycle shows that cracking occurs to $\Delta \mathrm{K}=2-3 \mathrm{MPa} \sqrt{\mathrm{m}}$ for low $\mathrm{P}_{\mathrm{H} 2 \mathrm{O}}$. Following a test protocol that uses a crack growth threshold of $1.0 \mathrm{E}-7 \mathrm{~mm} /$ cycle will help to reduce the chance of using non-conservative growth rates. Additionally, these experiments show that component geometry influences the molecular flow path and by extension, crack growth rates for a given stress intensity factor. From the principle of similitude, fatigue cracks grow at an equal rate $(\mathrm{da} / \mathrm{dN})$ when subjected to equal $\Delta \mathrm{K}$, implying that the stress intensity factor range uniquely defines the crack-tip conditions. This principle allows experimental data taken from lab specimens to be used in life prediction for real components. These experimental 
results suggest that similitude is compromised by the environment and geometry dependency. To mitigate for this concern, test specimens should be selected that are representative (similar flow path) of the airframe component to be modeled. Following these testing procedures would help to ensure data that accurately represent the increased fatigue resistance of 7075-T651 and 2199-T86 at high altitudes.

\section{Conclusions}

Compliance-based characterization of 7075-T651 and 2199-T86 C(T) specimens fatigued under $\Delta \mathrm{K}$-shed and constant $\Delta \mathrm{K}$ loading protocols for various high purity $\mathrm{P}_{\mathrm{H} 2 \mathrm{O}}$ exposures representative of high altitudes yielded a novel set of fatigue crack growth rates. Lower exposures exhibited a novel threshold transition regime that was analyzed with respect to prominent environmental fatigue theories. The following conclusions are established:

- The TTR behavior is an environmental effect; specifically associated with the enhanced transport of water vapor molecules from the bulk environment to the crack tip, this process is dependent on crack wake history and bulk $\mathrm{P}_{\mathrm{H} 2 \mathrm{O}}$

- Constant $\Delta \mathrm{K}, \mathrm{P}_{\mathrm{H} 20} / f$ experiments served to show that the interaction between the roughness on the fracture surface and the molecular transport can result in an order of magnitude change in growth rates directly following crack growth of increased roughness. Additionally, fractography suggests an environmentally driven irregular crack front driven by the interaction of molecular transport, closure, asperity based mixing, and position dependent $\Delta K$. 
- The pertinent diffusion distance for molecular transport to the crack tip is the through thickness direction, as such, changing the specimen geometry reasonably influences the environmental crack behavior at intermediate exposures.

- A quantitative method to indicate the presence of crack wake asperity contact based on comparing the localized roughness and crack opening displacement was developed. These results suggest that the start of crack wake asperity contact does not correspond directly to an increase in da/dN; counter to the proposed molecular transport hypothesis which stated that enhanced crack growth rates corresponded to the start of crack wake asperity contact occurring. The chosen contact metric indicates the presence of crack wake asperity contact, but the role it plays in controlling the upturn in da/dN rates until the Mode II displacement occurring is accurately measured. These results do suggest that the upturn in $\mathrm{da} / \mathrm{dN}$ is driven by the interaction of molecular transport, closure, asperity based mixing, and position dependent $\Delta \mathrm{K}$.

- Despite different global cracking morphology, the similarities in the scope and span of the roughness transition in 2199-T86 suggest a similar threshold transition regime that depends on $\Delta \mathrm{K}$ and the environment similar to the molecular transport based theory developed for 7075-T65.

Analysis detailed above indicates that the threshold transition behavior observed in 7075-T651 and 2199-T86 is governed by molecular transport that can be affected by the loading protocol, specimen geometry, and/or testing configuration. While the false threshold behavior resulting in the dip in da/dN is real and repeatable, such behavior is geometry and molecular flow path dependent and should not be incorporated into fracture mechanics based predictions. Following 
appropriate testing procedures would help to ensure data that accurately represent the increased fatigue resistance of 7075-T651 and 2199-T86 at high altitudes.

\section{Acknowledgements}

Funding for this work was provided by ALCOA and the US Office of the Secretary of Defense Corrosion Office via the Technical Corrosion Collaboration. These contributions are gratefully acknowledged. The views and conclusions contained herein are those of the author and should not be interpreted as necessarily representing the official polices and endorsements, either expressed or implied of the US Air Force Academy or the US Government.

I would like to personally thank Dr. James T. Burns for his guidance and mentorship for the past two years. A huge thank you to all the members of the Fracture Group who have helped me every day of my graduate school career. Finally, I would have never been able to achieve this goal without my family and friends. Thank you for being there for me every step of the way.

\section{References}

1. Burns JT, et al. Effect of water vapor pressure on faituge crack growth in $\mathrm{Al}-\mathrm{Zn}-\mathrm{Cu}-\mathrm{Mg}$ over wide-range stress intensity factor loading. Eng Fract Mech. 2015; http://dx.doi.org/10.1016/j.engfracmech.2014.11.009

2. Paris PC, Erdogan F. A critical analysis of crack propagation laws. J Basic Engr Trans ASME, Series D. 1963;85:528-34.

3. Vasudevan AK, Sadananda K, Louat N. Two critical stress intensities for threshold fatigue crack-propagation. Scripta Metall Mater. 1993;28:65-70.

4. Dinda S, Kujawski D. Correlation and prediction of fatigue crack growth for different R-ratios using K-max and $\Delta \mathrm{K}$ parameters. Eng Fract Mech. 2004;71:1779-90.

5. Vasudevan AK, Sadananda K. A viewpoint on environmental contributions to fatigue crack growth. Int J Mater Prod Tec. 2007;30:3-16.

6. Vasudevan AK, Sadananda K, Glinka G, Kujawski D. System and method for predicting material fatigue and damage. United States patent US 78898402011 Feb 15.

7. Bozek JE, Hochhalter JD, Veilleux MG, Liu M, Heber G, Sintay SD, et al. A geometric approach to modeling microstructurally small fatigue crack formation: I. Probabilistic simulation of constituent particle cracking in AA 7075-T651. Model Simul Mater Sci. 2008; 16:1-28.

8. Handbook for Damage Tolerant Design [Internet]. US Air Force Research Lab; c2009-11. Available from: http://www.afgrow.net/applications/DTDHandbook/.

9. Burns JT, Gangloff RP. Scientific advances enabling next generation management of corrosion induced fatigue. Procedia Eng. 2011;10:362-9.

10. Wei RP, Gangloff RP. In: Wei RP, Gangloff RP, editors. Fracture mechanics: perspective and directions, ASTM STP 1020, West Conshohocken, PA: ASTM Int; 1989. p. 233-64. 
11. Aicher W, Branger J, Van Dijk GM, et al. Description of a fighter aircraft loading standard for fatigue evaluation "FALSTAFF". Common Report of F+W Emmen, LBF, NLR, IABG. 1976.

12. Schutz D, Gerharz J. Enstaff: A standard test sequence for composite components combining load and environment. New materials and fatigue resistant aircraft. Proceedings of the $14^{\text {th }}$ Symposium of the International Committee on Aeronautical Fatigue. 1987; 425-444.

13. Burns, JT. Analysis and modeling of high-strength aluminum fatigue in airframe environments: UVA/ALCOA collaboration white paper. 2011.

14. Aronstein DC, Piccirillo AC. The lightweight fighter program: A successful approach to fighter technology transition. Arlington, VA: ANSER, Inc. 1997.

15. Smith MA, King HF. Airframe fatigue: A.R.B.'s chief technical officer gives the second Barnwell Lecture. Flight and Aircraft Engineers. 1955;67:347-352.

16. Harper RHT. Fatigue in aircraft structures. The New Scientist. 1957; 2: 23-25.

17. de Jonge JB, Spiekhout DJ. Use of AIDS recorded data for assessing service load experience. In: Abelkis PR, Potter JM, eds. Service Fatigue Loads Monitoring, Simulation, and Analysis, ASTM STP 671. ASTM West Conshohocken, PA, 1979. p 48-66.

18. Wei RP. Fracture mechanics: integration of mechanics, materials science, and chemistry. Cambridge, England: Cambridge University Press; 2010.

19. Petit J, Henaff G, Sarrazin-Baudoux C. Environmentally assisted fatigue in the gaseous atmosphere. In: Petit J, Scott P, editors. Comprehensive structural integrity: environmentally assisted fracture, New York: Elsevier; 2003. p. 962-70.

20. Petit J, Fouquet JD, Henaff G. In: Carpinteri A, editor. Handbook of fatigue crack propagation in metallic structures, Amsterdam, Netherlands: Elsevier Science; 1994. p. 1159203.

21. Gangloff RP. Environment sensitive fatigue crack tip processes and propagation in aerospace aluminum alloys. In: Blom A, editor. Fatigue 2002, Stockholm, Sweden: EMAS; 2002, p.

22. Bradshaw FJ, Wheeler C. The influence of gaseous environment and fatigue frequency on the growth of fatigue cracks in some aluminum alloys. Intl J of Fracture Mech. 1969;5:25562.

23. Turnbull A. Modeling of the chemistry and electrochemistry in cracks - a review. Corrosion. 2001;57:175-89.

24. Wiederhold PR. Water vapor measurement methods and instrumentation. New York, NY: CRC Press; 1997.

25. Burns JT. Effect of water vapor pressure on the fatigue crack propagation of aerospace aluminum alloys 7075-T651 and 2199-T86. In: Proceedings of NACE DoD Corrosion Conference, NACE International. 2013.

26. Burns JT, Gangloff RP. Effect of loading environment on fatigue crack formation and microstructurally small fatigue propagation in $\mathrm{Al}-\mathrm{Zn}-\mathrm{Mg}-\mathrm{Cu}$. Metall Mater Trans A. 2013; 44A: 2083-2105.

27. Ranganathan N, McKeighan PC. Fatigue testing and analysis under variable amplitude loading conditions, STP 1439. West Conshohocken, PA: ASTM International; 2005.

28. Skinn DA, Gallagher JP, Berens AP, Huber PD, Smith J. WL-TR-94-0452. Wright-Patterson AFB, OH: AFRL/Materials Directorate; 1994.

29. Burns JT, Bush RW, Gangloff RP. The effect of environment on corrosion induced fatigue crack formation and early propagation in Al 7075-T651. In: Proceedings of NACE DoD Corrosion Conference, NACE International. 2011. 
30. Weir TW, Simmons GW, Hart RG, Wei RP. Model for surface-reaction and transport controlled fatigue crack-growth. Scripta Metall Mater. 1980;14:357-64.

31. Wei RP, Pao PS, Hart RG, Weir TW, Simmons GW. Fracture-mechanics and surfacechemistry studies of fatigue crack-growth in an aluminum-alloy. Metall Mater Trans A. 1980;11:151-8.

32. Achter MR. The adsorption model for environmental effects in fatigue crack propagation. Scripta Metall Mater. 1968;2:525-7.

33. Wei RP. Environmental considerations for fatigue cracking. Fatigue Fract Eng Mater Struct. 2002;25:845-54.

34. Gao M, Pao PS, Wei RP. Chemical and metallurgical aspects of environmentally assisted fatigue crack-growth in 7075-T651 aluminum-alloy. Metall Mater Trans A. 1988;19:173950.

35. Pao PS, Gao M, Wei RP. Critical assessment of the model for transport-controlled fatigue crack growth. In: Wei RP, Gangloff RP, editors. Basic questions in fatigue, ASTM STP 92, West Conshohocken, PA: ASTM Int; 1988. p. 182-95.

36. Pao PS, Gao MN, Wei RP. Environmentally assisted fatigue-crack growth in 7075 and 7050 aluminum-alloys. Scripta Metall Mater. 1985;19:265-70.

37. Wei RP, Gao M, Pao PS. The role of magnesium in CF and SCC of 7000 series aluminum alloys. Scripta Metall Mater. 1984;18:1195-8.

38. Henaff G, Marchal K, Petit J. On fatigue-crack propagation enhancement by a gaseous atmosphere - experimental and theoretical aspects. Acta Metall Mater. 1995;43:2931-42.

39. Ruiz J, Elices M. Effect of water vapour pressure and frequency on fatigue behaviour in 7017-T651 aluminium alloy plate. Acta Metall Mater. 1997;45:281-93.

40. Ro YJ, Agnew SR, Bray GH, Gangloff RP. Environment-exposure-dependent fatigue crack growth kinetics for Al-Cu-Mg/Li. Materials Science and Engineering A. 2007; 468: 88-97.

41. Giummarra C, Rioja RJ, Bray GH, Magnusen PE, Moran JP. Al-Li alloys: Development of corrosion resistant, high toughness, aluminum-lithium aerospace alloys. In: Hirsh J, Skrotzki B, Gottstein G, eds. Aluminum Alloys: Their Physical and Mechanical Properties. Weinheim, Germany:Wiley-VCH; 2008.

42. Rao KTV, Yu W, Ritchie RO. Fatigue crack-propagation in aluminum-lithium alloy 2090 part 2. Small crack behavior. Metall Trans A. 1988; 19: 563-569.

43. Rao KTV, Yu W, Ritchie RO. Fatigue crack-propagation in aluminum-lithium alloy 2090 part 1. Long crack behavior. Metall Trans A. 1988; 19: 549-561.

44. Piascik RS, Gangloff RP. Environmental fatigue of an Al-Li-Cu alloy 1. Intrinsic crack propagation kinetics in hydrogenous environments. Metall Trans A. 1991; 22: 2415-2428.

45. ASTM E647-08: Standard test method for measurement of fatigue crack growth rates, Vol. 03.01. West Conshohocken, PA: ASTM International; 2013.

46. Donald JK. Introducing the compliance ratio concept for determining effective stress intensity. Int J Fatigue. 1997;19:S191-S195.

47. McClung RC, Davidson, DL. Near-tip and remote characterization of plasticity-induced fatigue crack closure, in: R.C. McClung, J.C.J. Newman (Eds.) Advances in fatigue crack closure measurement and analysis, ASTM STP 1343. West Conshohocken, PA: ASTM International. 1999; 106-127.

48. Graham SM, Tregoning R, Zhang XJ. Evaluation of the adjusted compliance ratio technique for measuring crack closure in Ti-6Al-4V, in: R.C. McClung, J.C. Newman, Jr. (Eds.) 
Advances in Fatigue Crack Closure Measurement and Analysis: ASTM STP 1343. ASTM

STP 1343. West Conshohocken, PA: ASTM International. 1999; 94-105.

49. Ro Y, et al. Environment-exposure-dependent fatigue crack growth kinetics for $\mathrm{Al}-\mathrm{Cu}-$ Mg/Li. Materials Science and Engineering: A. 2007; 468-470:88-97.

50. Ro Y, Agnew SR, Gangloff RP. Environmental fatigue-crack surface crystallography for AlZn-Cu-Mg-Mn/Zr. Metall Mater Trans A. 2008; 39A:1449-65.

51. Sunder R, Porter WJ, Ashbaugh NE. Fatigue voids and their significance. Fatigue Fract Eng Mater Struct. 2002;25:1015-24.

52. Aerospace Engineering Guide: Surface Finish [Internet]. Trelleborg Sealing Solutions; 2008. http://www.alphaomegapt.com/pdf\%20files/Surface\%20Finish\%20Definitions.pdf

53. ASTM E399-90: Standard test method for plane-strain fracture toughness of metallic materials, Vol. 03.01. West Conshohocken, PA: ASTM International; 2013.

54. Piascik RS, Gangloff RP. Environmental fatigue of an Al-Li-Cu alloy. Part 2: Microscopic hydrogen cracking processes. Metall Trans A. 1993; 24: 2751-2762.

55. Newman JC, Anagnostou EL, Rusk DT. Fatigue and crack-growth analyses on 7075-T651 aluminum alloy coupons under constant- and variable-amplitude loading. Int J Fatigue 2014; 62:133-43.

56. Turnbull A. Theoretical-analysis of influence of crack dimensions and geometry on masstransport in corrosion-fatigue cracks. Mater Sci Tech. 1985;1:700-10.

57. Cussler EL. Diffusion: mass transfer in fluid systems. 2nd ed. Cambridge, UK: Cambridge University Press; 1984.

58. Somerday BP, Sofronis P, Nibur KA, San Marchi C, Kirchheim R. Elucidating the variables affecting accelerated fatigue crack growth of steels in hydrogen gas with low oxygen concentrations. Acta Metall Mater 2013;61:6153-70.

59. Taunt RJ, Charnock W. Fluid compositions within fatigue cracks. Mater Sci Engng 1978;35:219-28.

60. Gombosi TI. Gas kinetic theory. Cambridge, UK: Cambridge University Press; 1994.

61. Kestin J, Knierim K, Mason EA, Najafi B, Ro ST, Waldman M. Equilibrium and transportproperties of the noble-gases and their mixtures at low-density. Phys Chem Ref Data 1984;13:229-303.

62. Thermopedia.com [Internet]. Begell House, Inc.; c2010-04, http://dx.doi.org/10.1615/AtoZ.d.diffusion_coefficient <http://www.thermopedia.com/ content/696/> [updated 10.02.11].

63. Dongari N, Sharma A, Durst F. Pressure-driven diffusive gas flows in micro-channels: from the Knudsen to the continuum regimes. Microfluid Nanofluid 2009;6:672-92.

64. Malek K, Coppens MO. Effects of surface roughness on self- and transport diffusion in porous media in the Knudsen regime. Phys Rev Lett 2001;87.

65. Malek K, Coppens MO. Knudsen self- and Fickian diffusion in rough nanoporous media. J Chem Phys 2003;119:2801-11.

66. Zschiegner S, Russ S, Bunde A, Karger J. Pore opening effects and transport diffusion in the Knudsen regime in comparison to self- (or tracer-) diffusion. Europhys Lett 2007;78:1-5.

67. Harley JC, Huang YF, Bau HH, Zemel JN. Gas-flow in micro-channels. J Fluid Mech 1995;284:257-74.

68. Sun HW, Faghri M. Effect of surface roughness on nitrogen flow in a microchannel using the direct simulation Monte Carlo method. Numer Heat Tr A - Appl. 2003;43:1-8. 
69. Garcia-Manrique J, Camas D, Lopez-Crespo P, Gonzalez-Herrera A. Stress intensity factor analysis of through thickness effects. Int J Fatigue. 2013; 46:58-66.

70. Hutar. P, Nahlik L, Knesl Z. The effect of a free surface on fatigue crack behavior. Int J Fatigue. 2010; 32:1265-1269.

71. Lin XB, Smith RA. Finite element modeling of fatigue crack growth of surface cracked plates. Part II: Crack shape change. Eng Frac Mec. 1990;63:523-540.

72. Koss DA, Chan KS. Fracture along planar slip bands. Acta Met. 1980; 28:9: 1245-1252.

73. Petit J, Sarrazin-Baudoux C. Some critical aspects of low rate fatigue crack propagation in metallic materials. Int J Fatigue. 2010;32:962-70.

74. Richard S, Gasqueres C, Sarrazin-Baudoux C, Petit J. Coupled influence of microstructure and atmosphere environment on fatigue crack path in new generation Al alloys. Eng Fract Mech. 2010;77:1941-52.

75. Newman JC. Crack-opening displacements in center-crack, compact, and crack-line wedgeloaded specimens. TN D-8286. NASA. 1976.

76. Saxena A, Hudak SJ. Review and extension of compliance information for common crack growth specimens. Int J of Fatigue. 1978; 14:453-467.

77. Anderson TL. Fracture mechanics: fundamentals and applications. 3rd ed. Boca Raton, FL: CRC Press; 1991.

78. Suresh S, Ritchie RO. A geometric model for fatigue crack closure induced by fracture surface roughness. Metall Mater Trans A. 1982; 13A: 1627-1631.

79. Lin XB, Smith RA. Finite element modeling of fatigue crack growth of surface cracked plates. Part III: Stress intensity factor and fatigue crack growth life. Eng Frac Mec. 1999;63:541-556.

80. Ro Y, Agnew SR, Gangloff RP. Crystallography of fatigue crack propagation in precipitationhardened Al-Cu-Mg/Li. Metall Mater Trans A. 2007; 38A:3042-62.

81. Giummarra C, Thomas B, Rioja R. New aluminum lithium alloys for aerospace applications. In: Proceedings of the Light Metals Technology Conference. 2007.

82. Branco R, Antunes FV, Martins RF. Modelling fatigue crack propagation in CT specimens. Fatigue Frac Eng Mater Struct. 2008;31:452-465. 


\section{Figures}

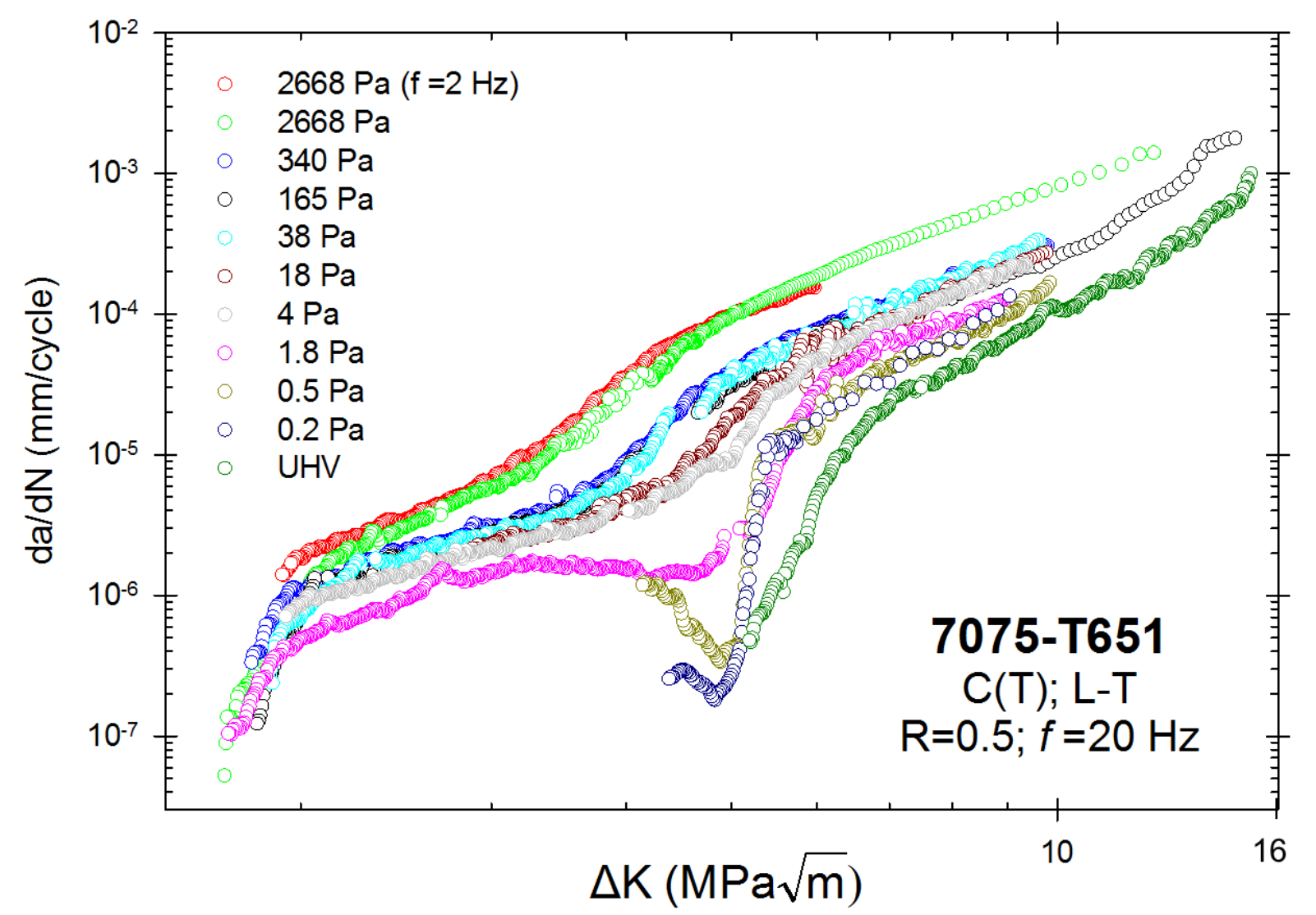

Figure 1: $\quad$ Fatigue crack growth rate versus decreasing $\Delta \mathrm{K}$ at constant $\mathrm{R}$ of $0.50(\mathrm{f}=20 \mathrm{~Hz})$ for 7075-T651 (L-T) at various-constant water vapor exposure levels from ultra-high vacuum $(0.25-0.50 \mu \mathrm{Pa}-\mathrm{s})$ to $1.3 \mathrm{kPa}-\mathrm{s}\left(\mathrm{P}_{\mathrm{H} 2 \mathrm{O}}=26.7 \mathrm{kPa}\right)[1]$. 


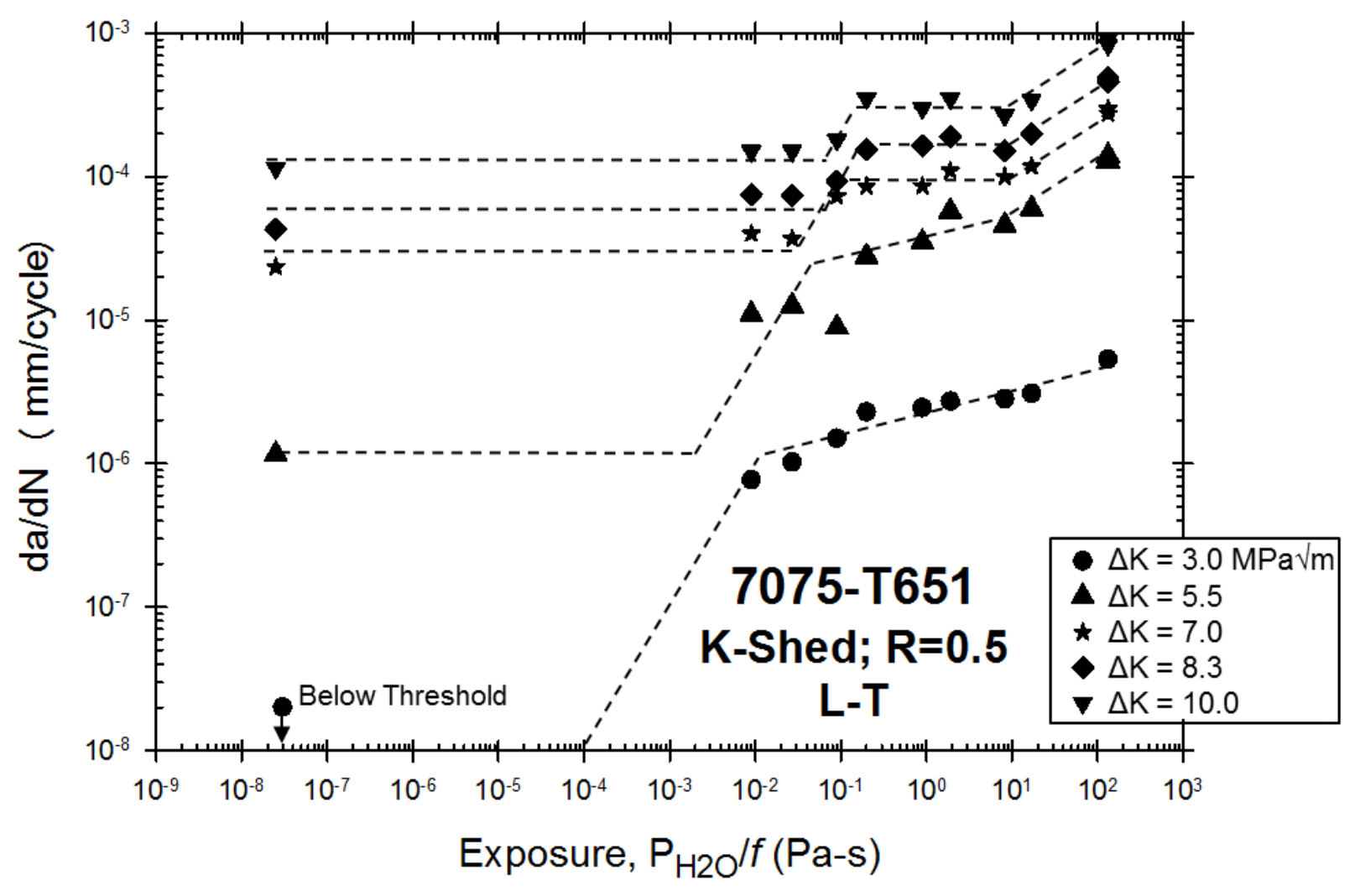

Figure 2: Effect of high purity water vapor exposure parameter on fatigue crack growth rate in 7075-T651 (L-T) stressed at various-constant $\Delta \mathrm{K}$ levels, each at fixed $\mathrm{R}$ of 0.50 and $\mathrm{f}=20 \mathrm{~Hz}$, taken from the wide-range decreasing $\Delta \mathrm{K}$ data in Figure $1[1]$. 

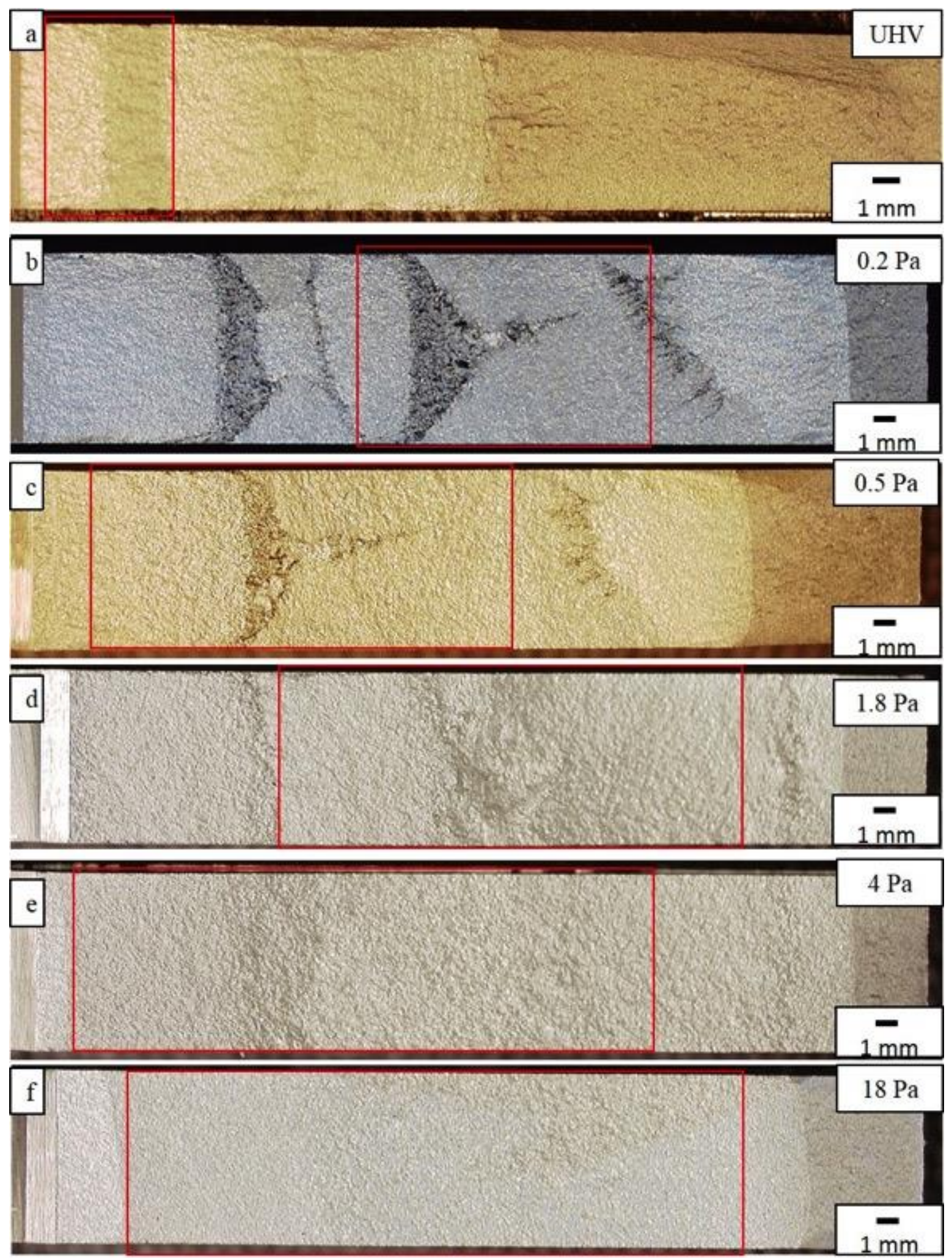


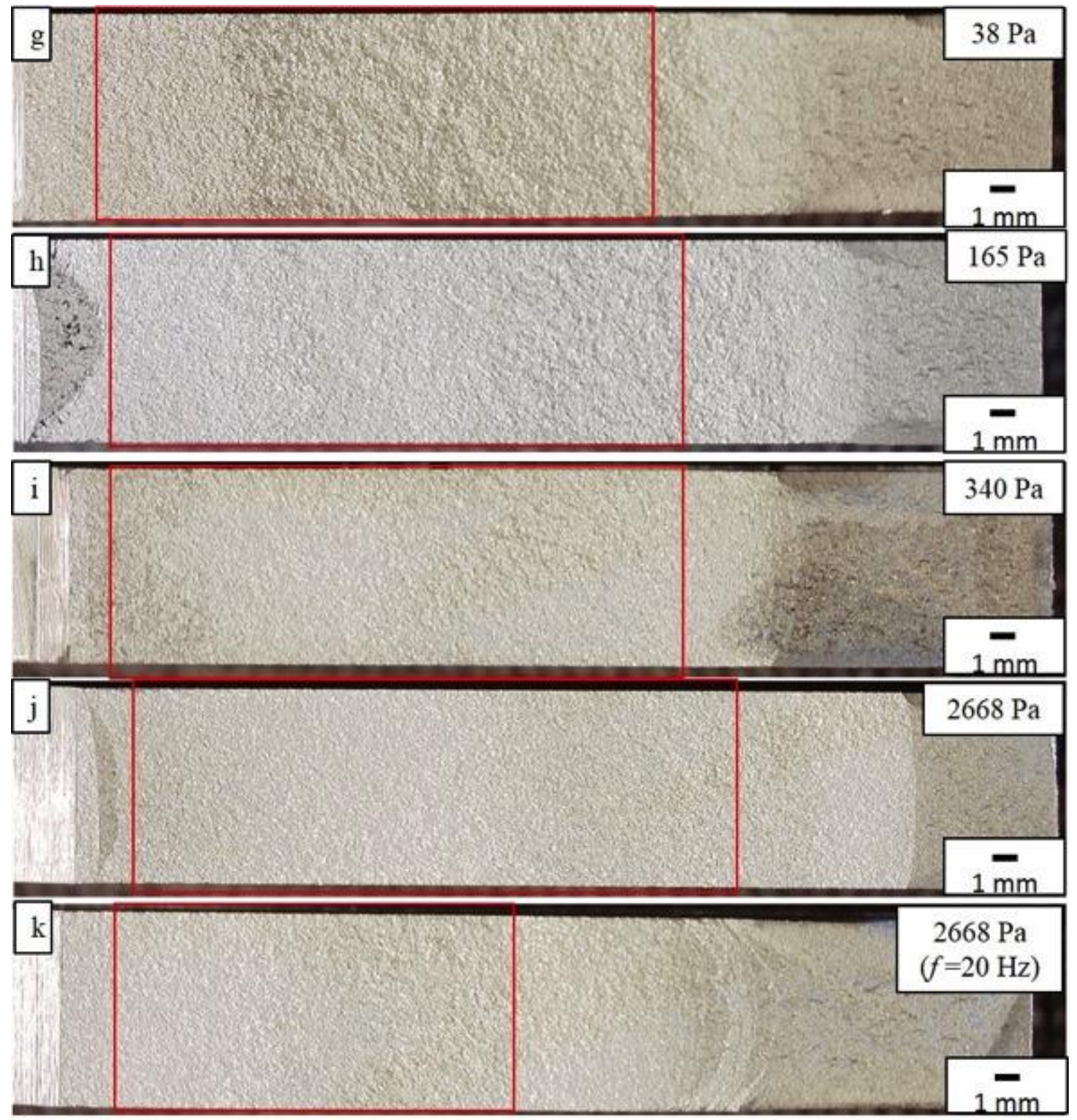

Figure 3: Optical fractographs for decreasing $\Delta \mathrm{K}$ at constant $\mathrm{R}$ of $0.50(\mathrm{f}=20 \mathrm{~Hz})$ for $7075-\mathrm{T} 651$ (L-T) at various-constant water vapor exposure levels: (a) Ultra-high vacuum, (b) 0.2, (c) 0.5, (d) 1.8, (e) 4, (f) 18, (g) 38, (h) 165, (i) 340, (j) 2668, and (k) $2668 \mathrm{~Pa} \mathrm{(f=2} \mathrm{Hz).} \mathrm{Crack} \mathrm{growth} \mathrm{is} \mathrm{from}$ left to right in each image. 

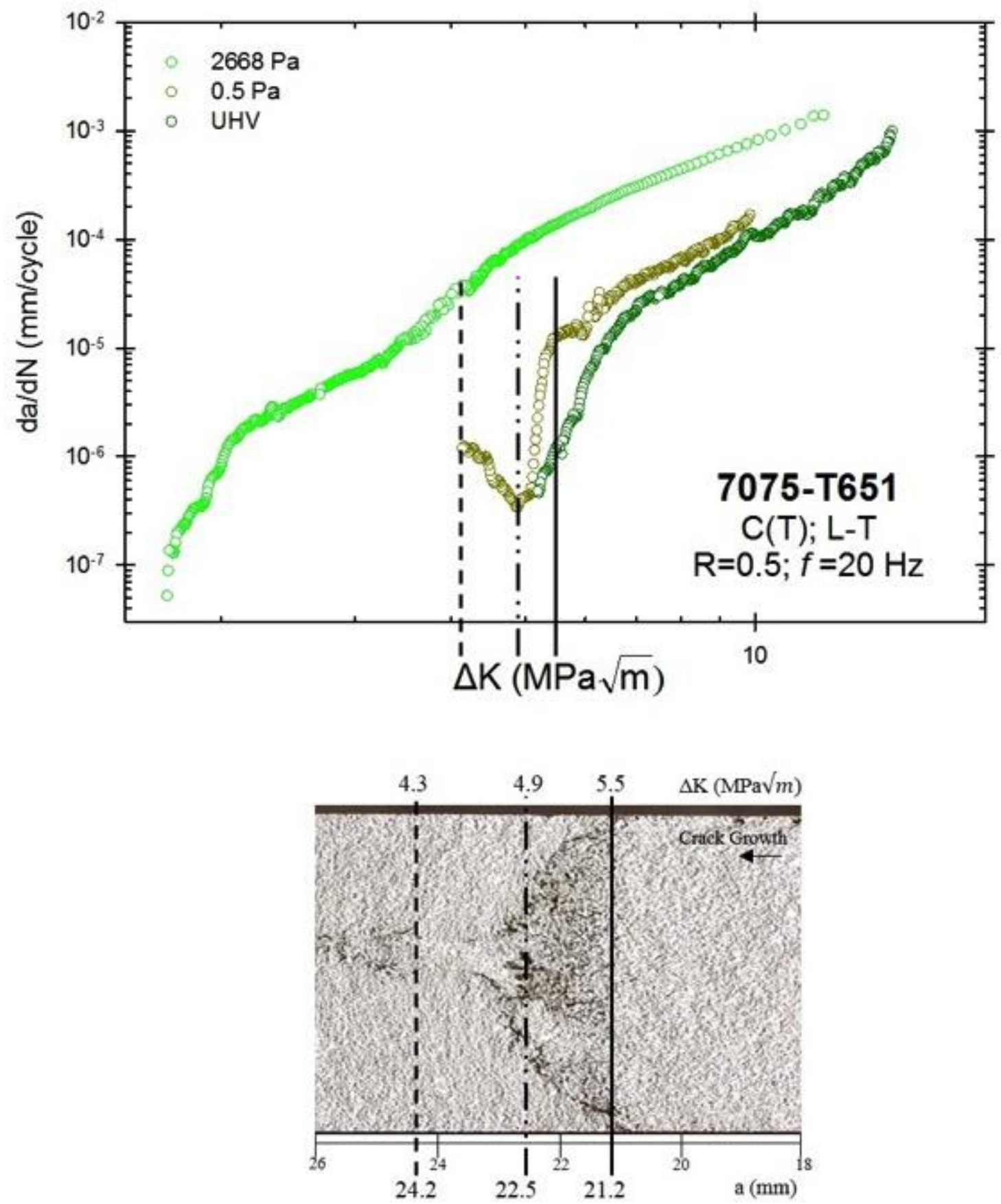

Figure 4: Crack growth rate versus $\Delta \mathrm{K}$ (a) and corresponding optical fractograph (b) illustrating the transition from macroscopically smooth to rough topography on the fatigue crack surface of 7075-T651 (L-T orientation) stressed in pure water vapor at $\mathrm{P}_{\mathrm{H} 2 \mathrm{O}}=0.5 \mathrm{~Pa}$. For constant $\mathrm{R}, \Delta \mathrm{K}$ decreased with increasing crack length from right to left in this image 

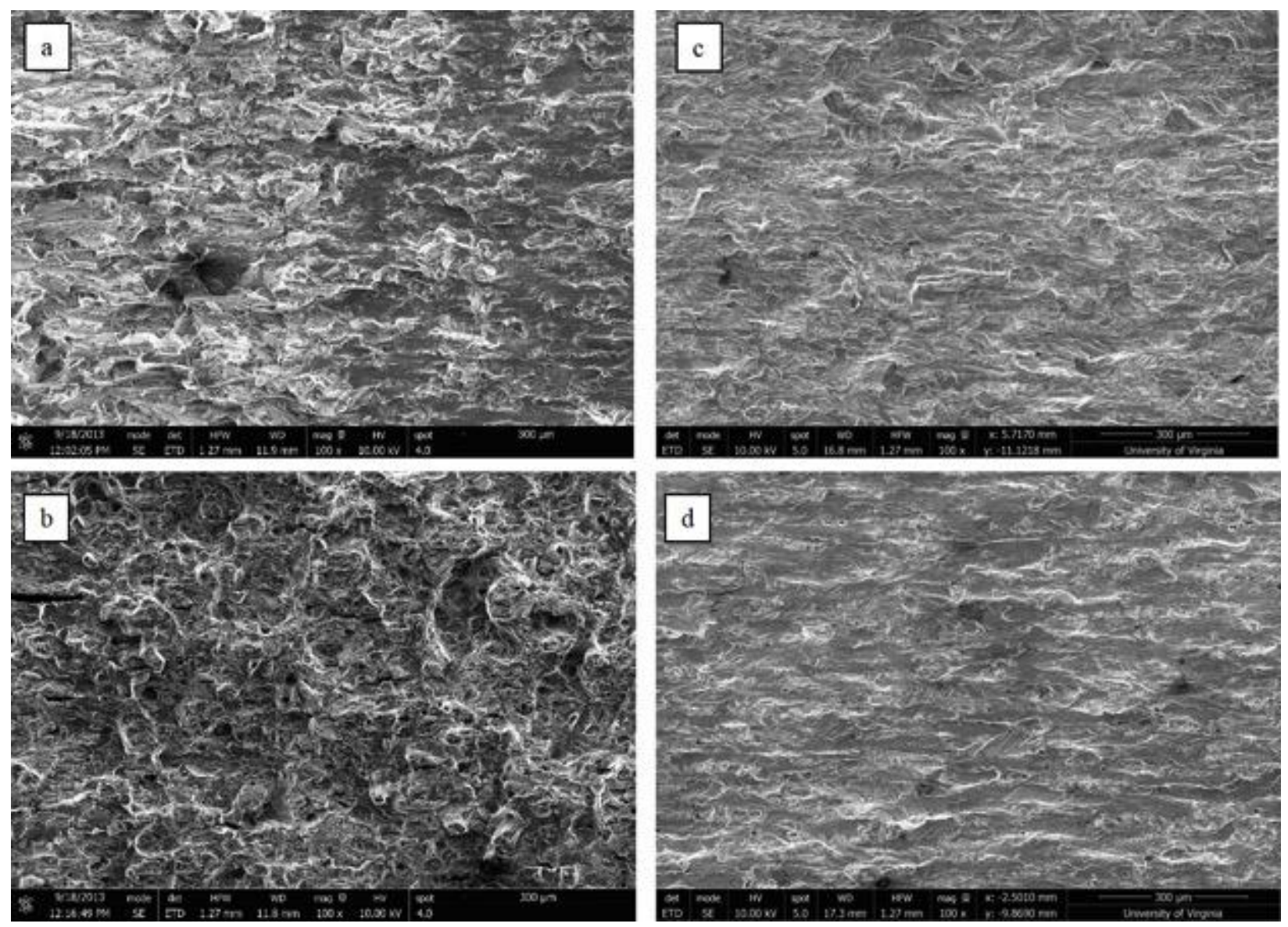

Figure 5: SEM images of the fatigue crack surface of 7075-T651 tested at constant $\mathrm{R}=0.5, f=20$ $\mathrm{Hz}$ (from Figure 1) for an L-T oriented specimen exposed to $\mathrm{P}_{\mathrm{H} 2 \mathrm{O}}$ of: UHV (a-b) and high humidity (c-d) with a $\Delta \mathrm{K}$ of: $(\mathrm{a}, \mathrm{c}) \sim 5,(\mathrm{~b}, \mathrm{~d}) \sim 9 \mathrm{MPa} \sqrt{\mathrm{m}}$. Crack growth is from left to right in each image. 

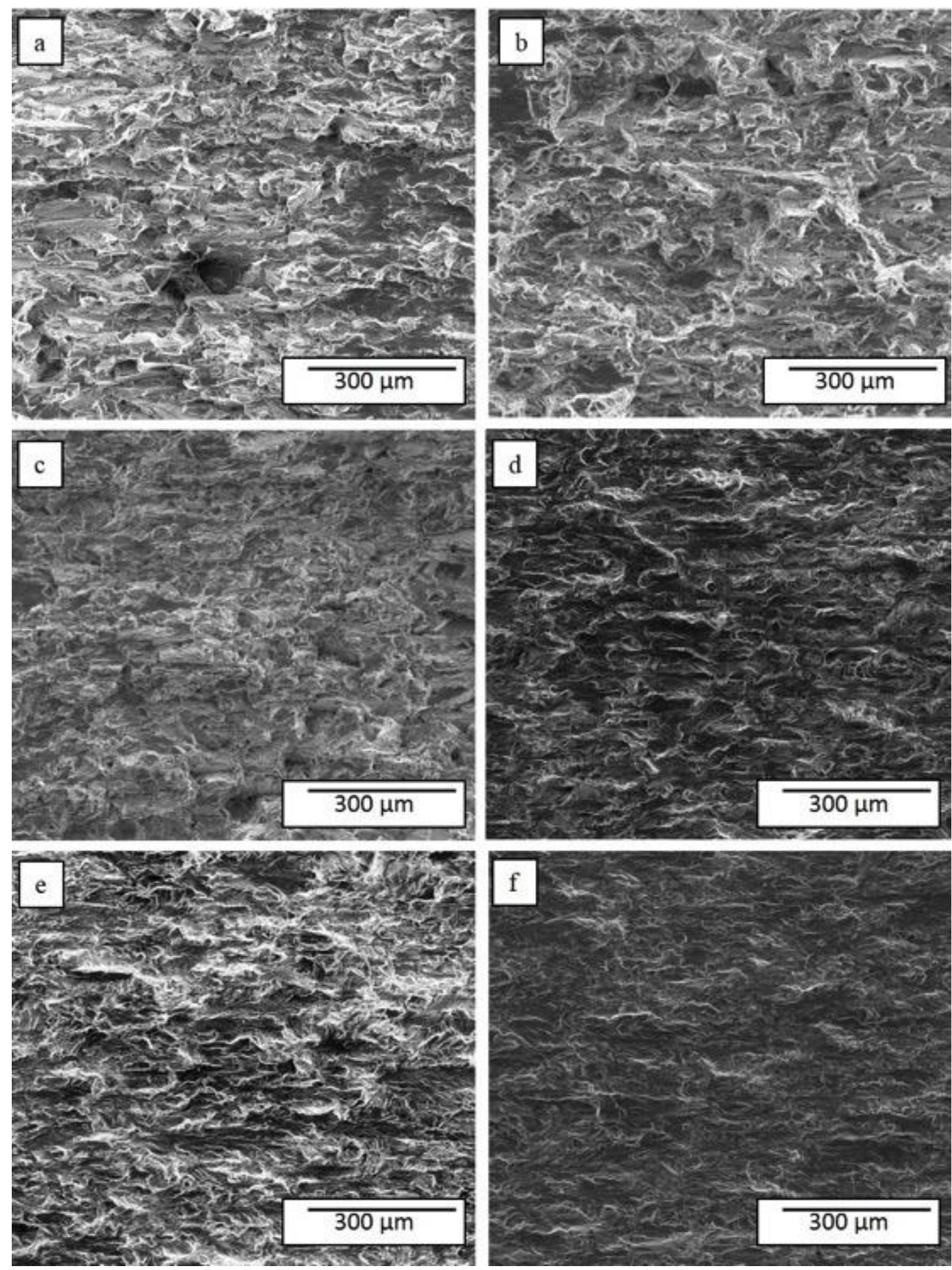

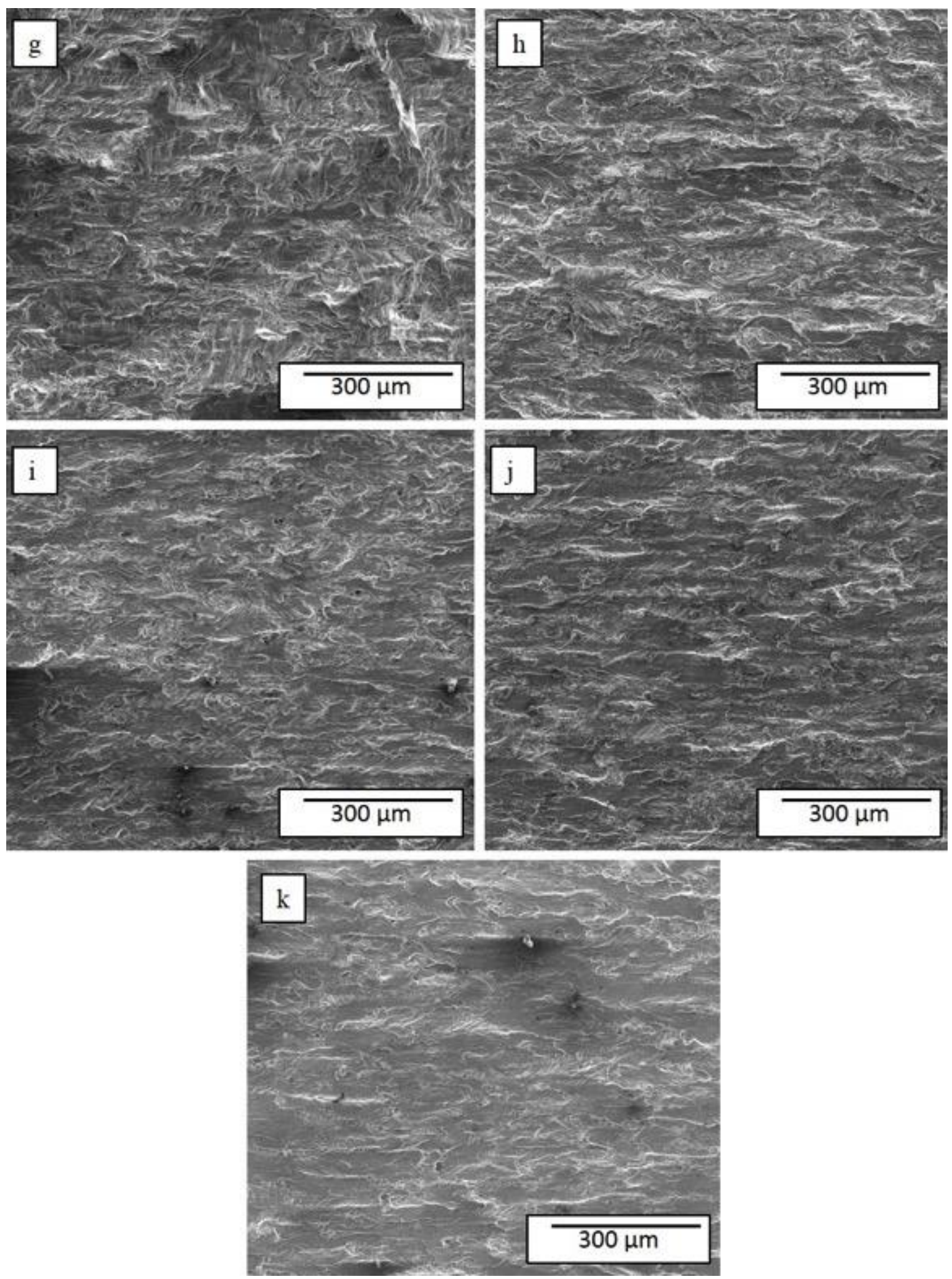

Figure 6: SEM images of the fatigue crack surface of 7075-T651 tested at constant $\mathrm{R}=0.5, \mathrm{f}=$ $20 \mathrm{~Hz}$ and constant $\Delta \mathrm{K}=5 \mathrm{MPa} \sqrt{\mathrm{m}}$ (from Figure 1) for an L-T oriented specimen exposed to PH2O of: (a) UHV (b) 0.2, (c) 0.5, (d) 1.8, (e) 4, (f) 18, (g) 38, (h) 165, (i) 340, (j) 2668, and (k) $2668 \mathrm{~Pa}(f=2 \mathrm{~Hz})$. Crack growth is from left to right in each image. 

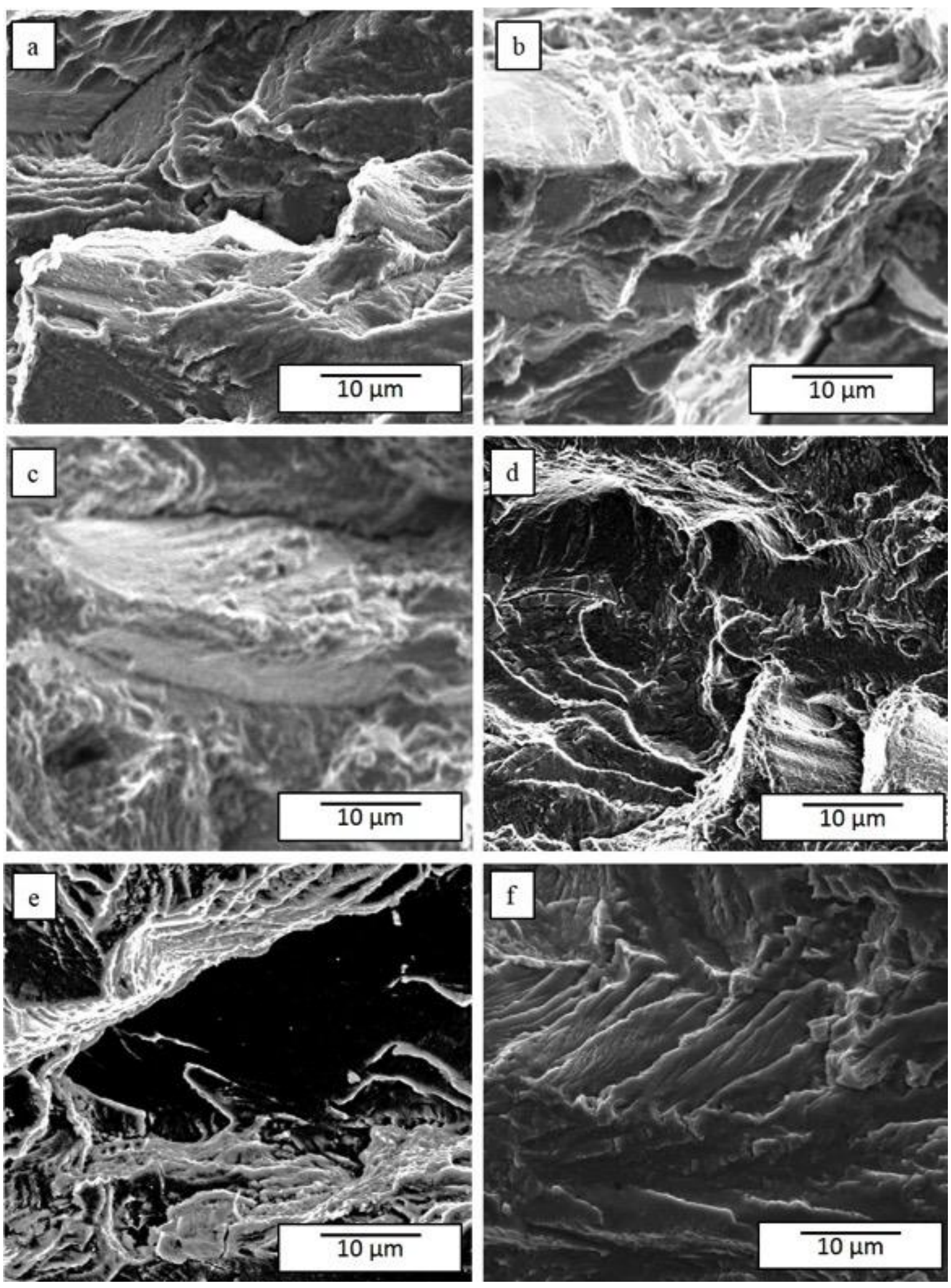

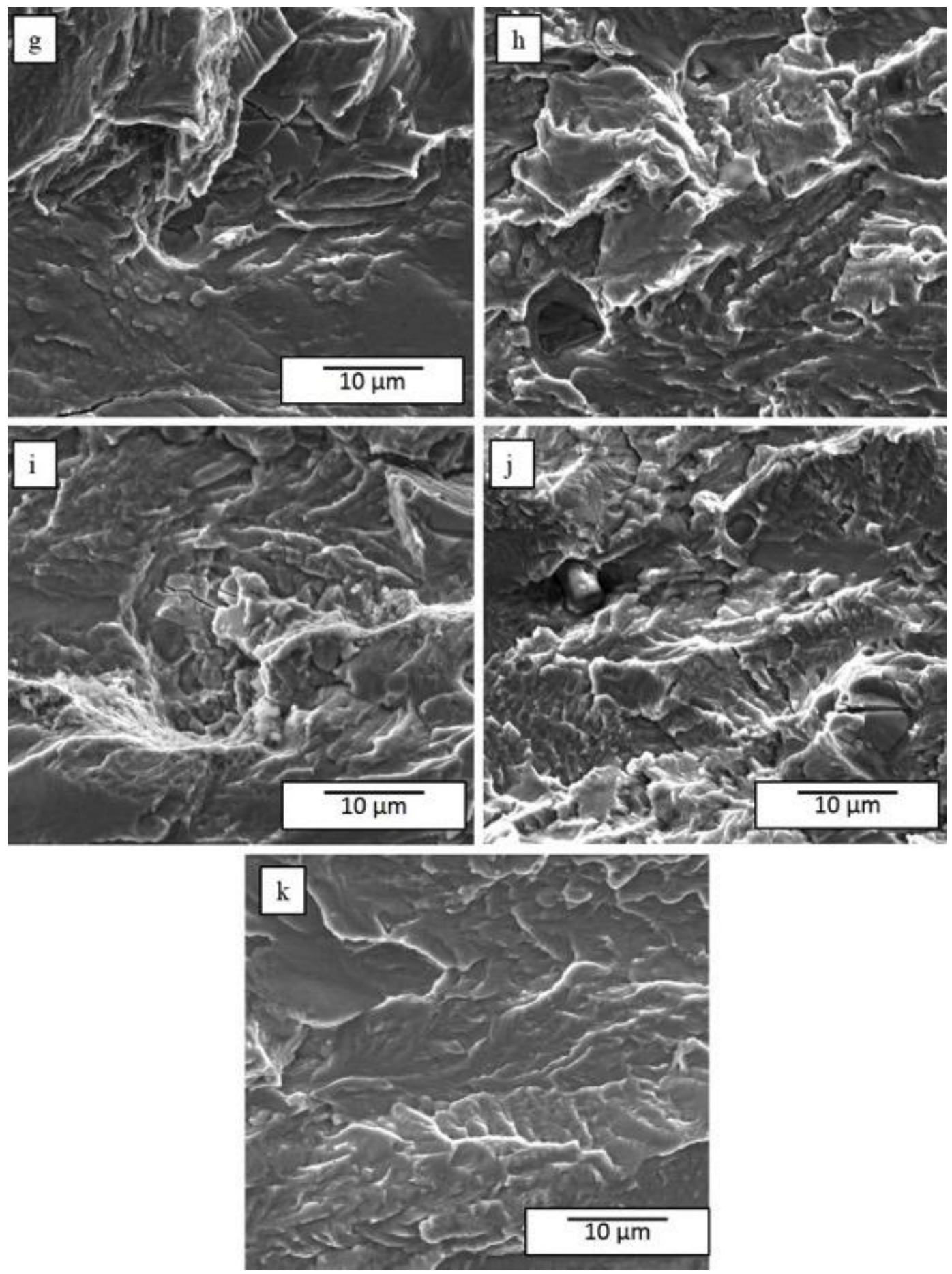

Figure 7: Higher magnification SEM images of the fatigue crack surface of 7075-T651 tested at constant $\mathrm{R}=0.5, f=20 \mathrm{~Hz}$ and constant $\Delta \mathrm{K}=5 \mathrm{MPa} \sqrt{\mathrm{m}}$ (from Figure 1 and Figure 8 ) for an LT oriented specimen exposed to $\mathrm{P}_{\mathrm{H} 2 \mathrm{O}}$ of: (a) UHV (b) 0.2, (c) 0.5, (d) 1.8, (e) 4, (f) 18, (g) 38, (h) 165, (i) 340, (j) 2668, and (k) $2668 \mathrm{~Pa}(\mathrm{f}=2 \mathrm{~Hz}$ ). Crack growth is from left to right in each image. 

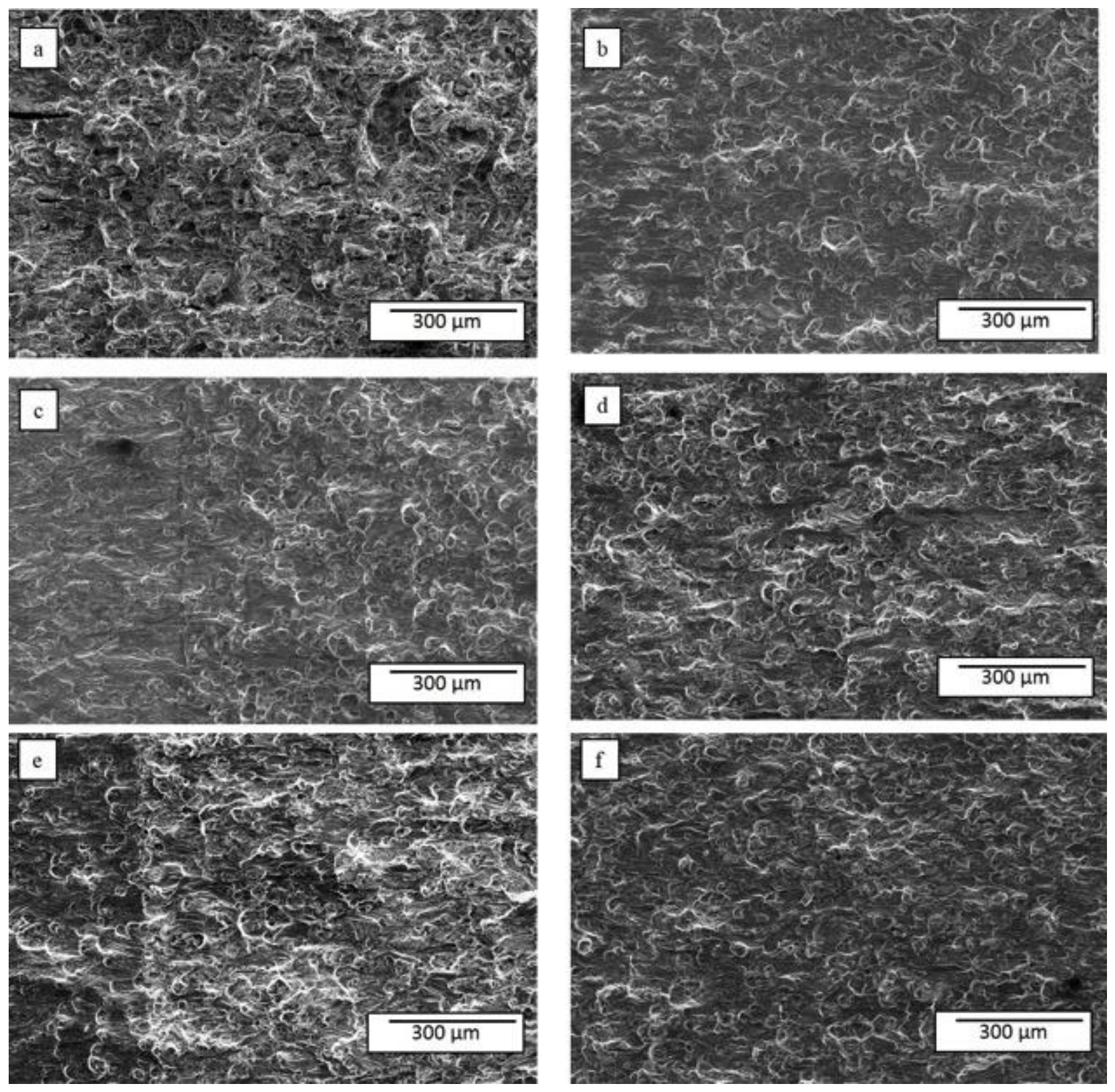

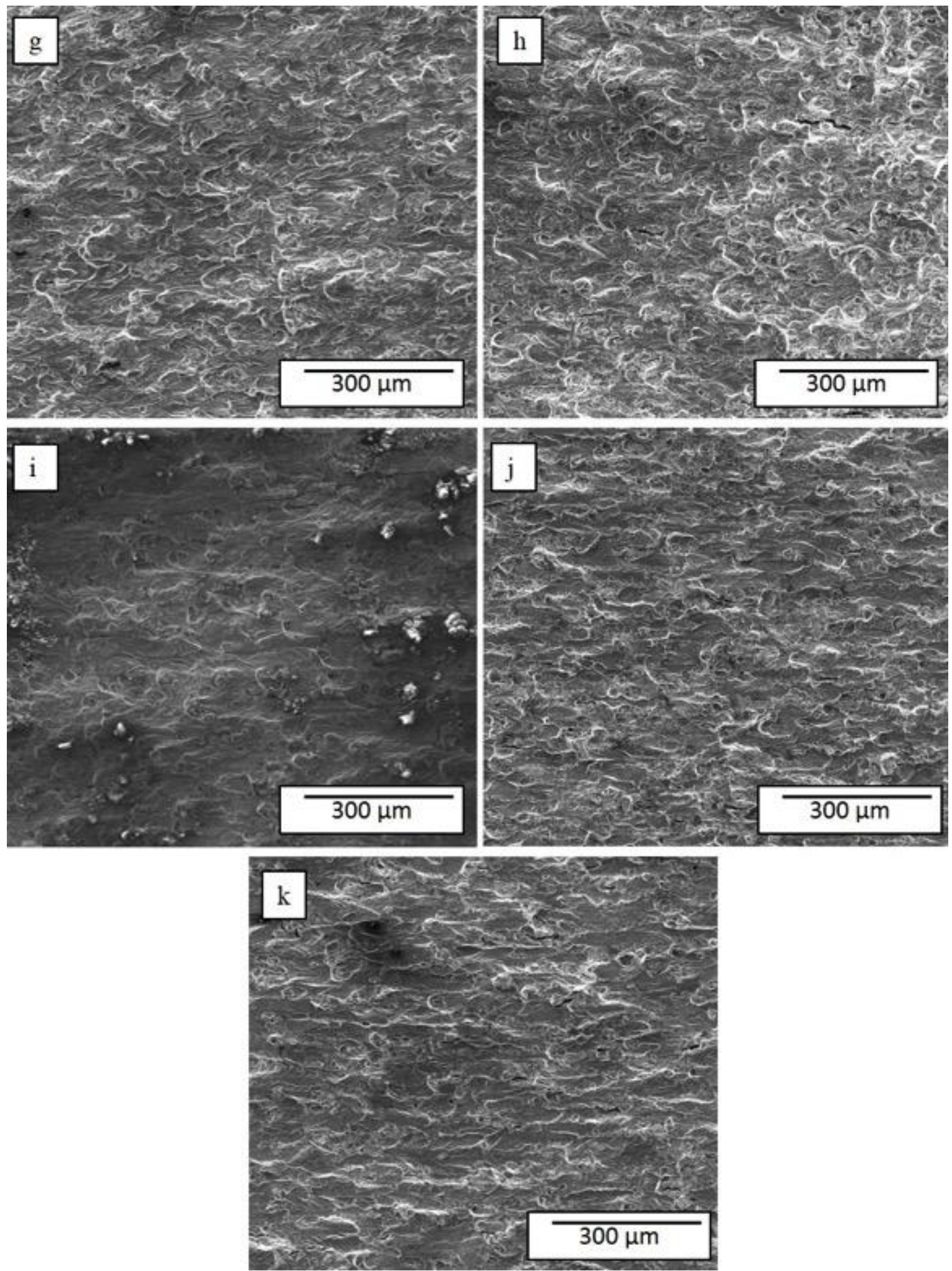

Figure 8: SEM images of the fatigue crack surface of 7075-T651 tested at constant $\mathrm{R}=0.5, f=$ $20 \mathrm{~Hz}$ and constant $\Delta \mathrm{K}=9 \mathrm{MPa} \sqrt{\mathrm{m}}$ (from Figure 1) for an L-T oriented specimen exposed to $\mathrm{P}_{\mathrm{H} 2 \mathrm{O}}$ of: (a) UHV (b) 0.2, (c) 0.5, (d) 1.8, (e) 4, (f) 18, (g) 38, (h) 165, (i) 340, (j) 2668, and (k) 2668 $\mathrm{Pa}(f=2 \mathrm{~Hz})$. Crack growth is from left to right in each image. 

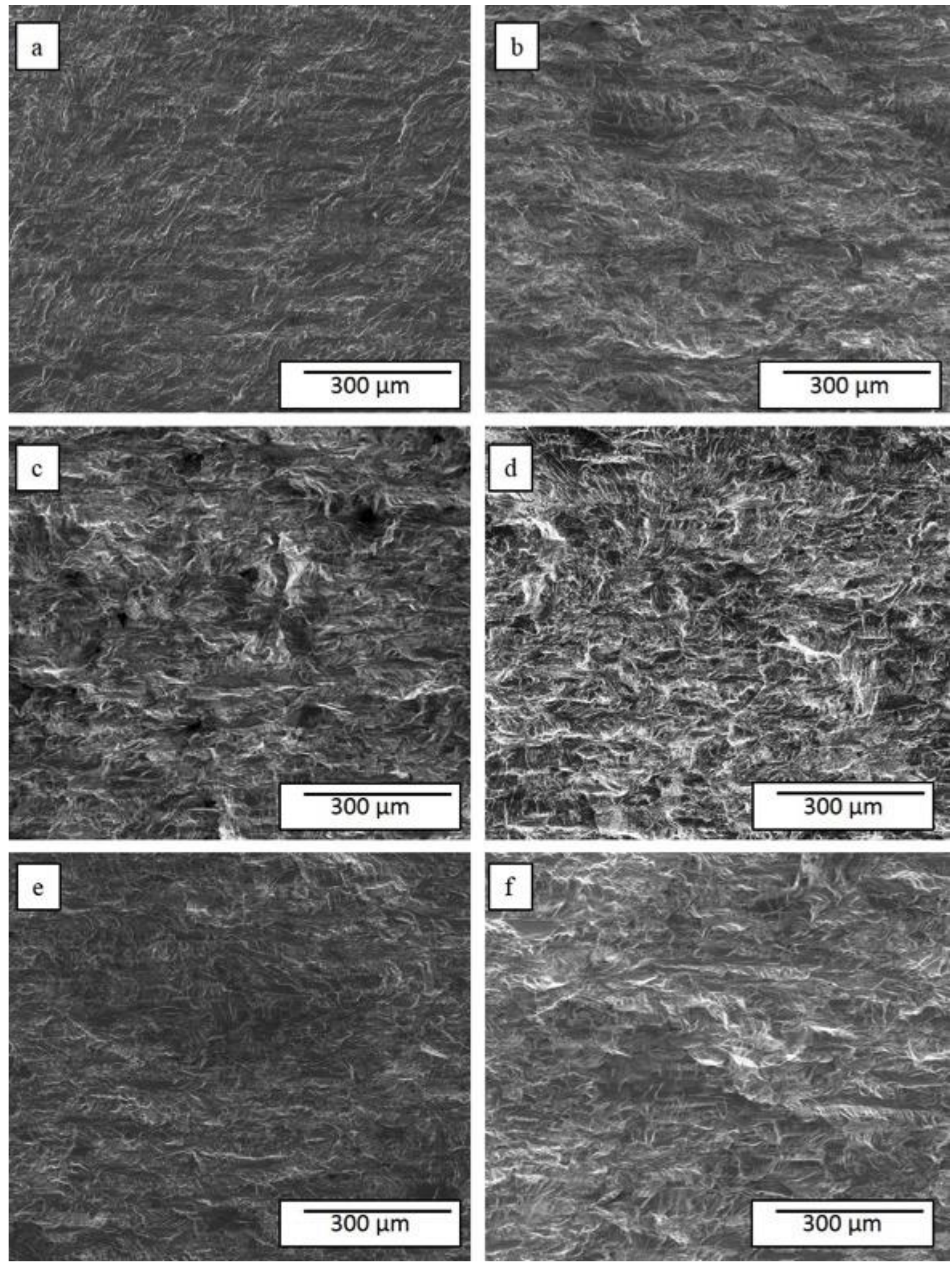

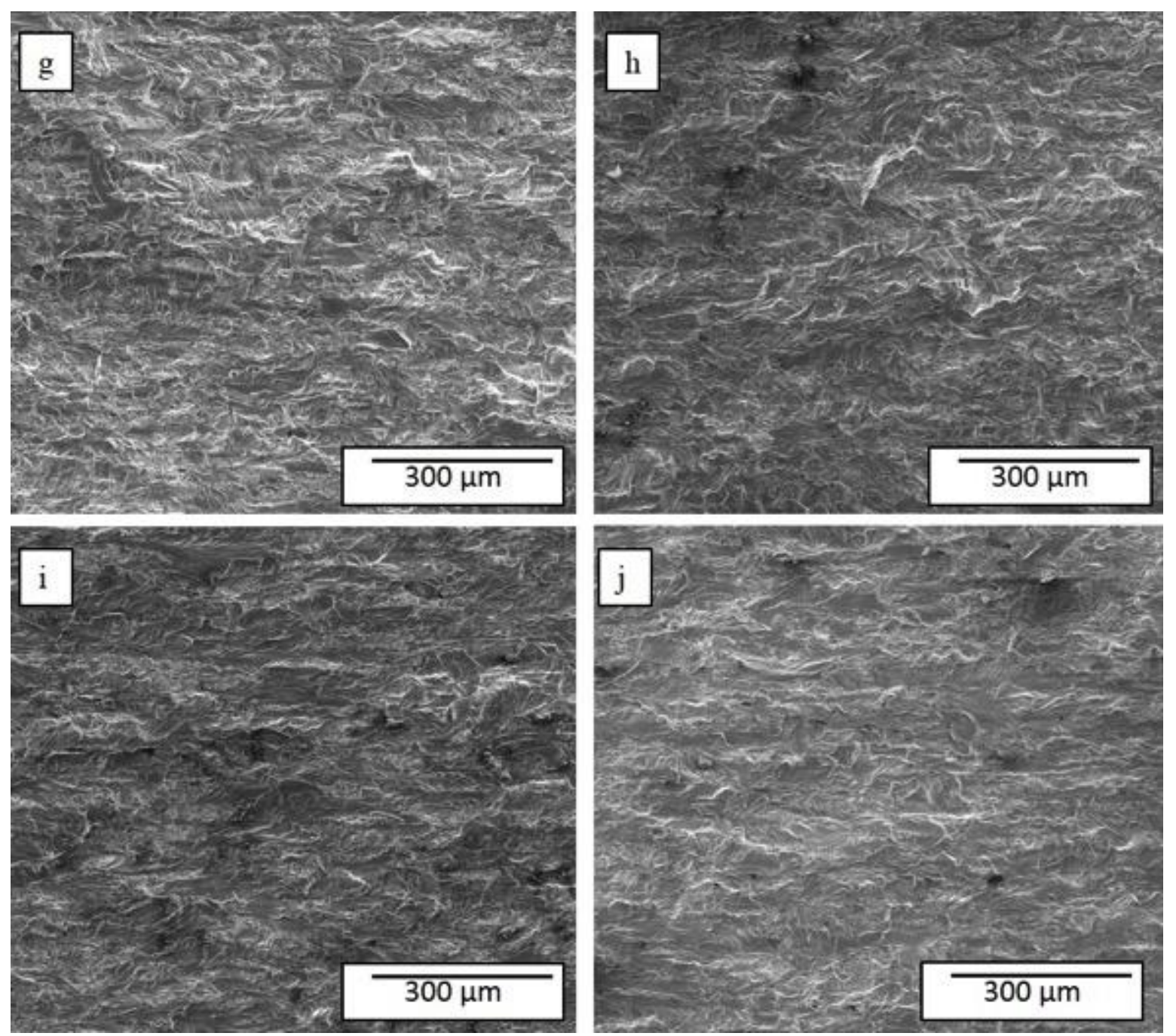

Figure 9: SEM images of the fatigue crack surface of 7075-T651 tested at constant $\mathrm{R}=0.5, f=$ $20 \mathrm{~Hz}$ and constant $\Delta \mathrm{K}=3 \mathrm{MPa} \sqrt{\mathrm{m}}$ (from Figure 1) for an L-T oriented specimen exposed to P $\mathrm{H} 2 \mathrm{O}$ of: (a) 0.2, (b) 0.5, (c) 1.8, (d) 4, (e) 18, (f) 38, (g) 165, (h) 340, (i) 2668, and (j) $2668 \mathrm{~Pa}$ (f $=2 \mathrm{~Hz}$ ). Crack growth is from left to right in each image. 

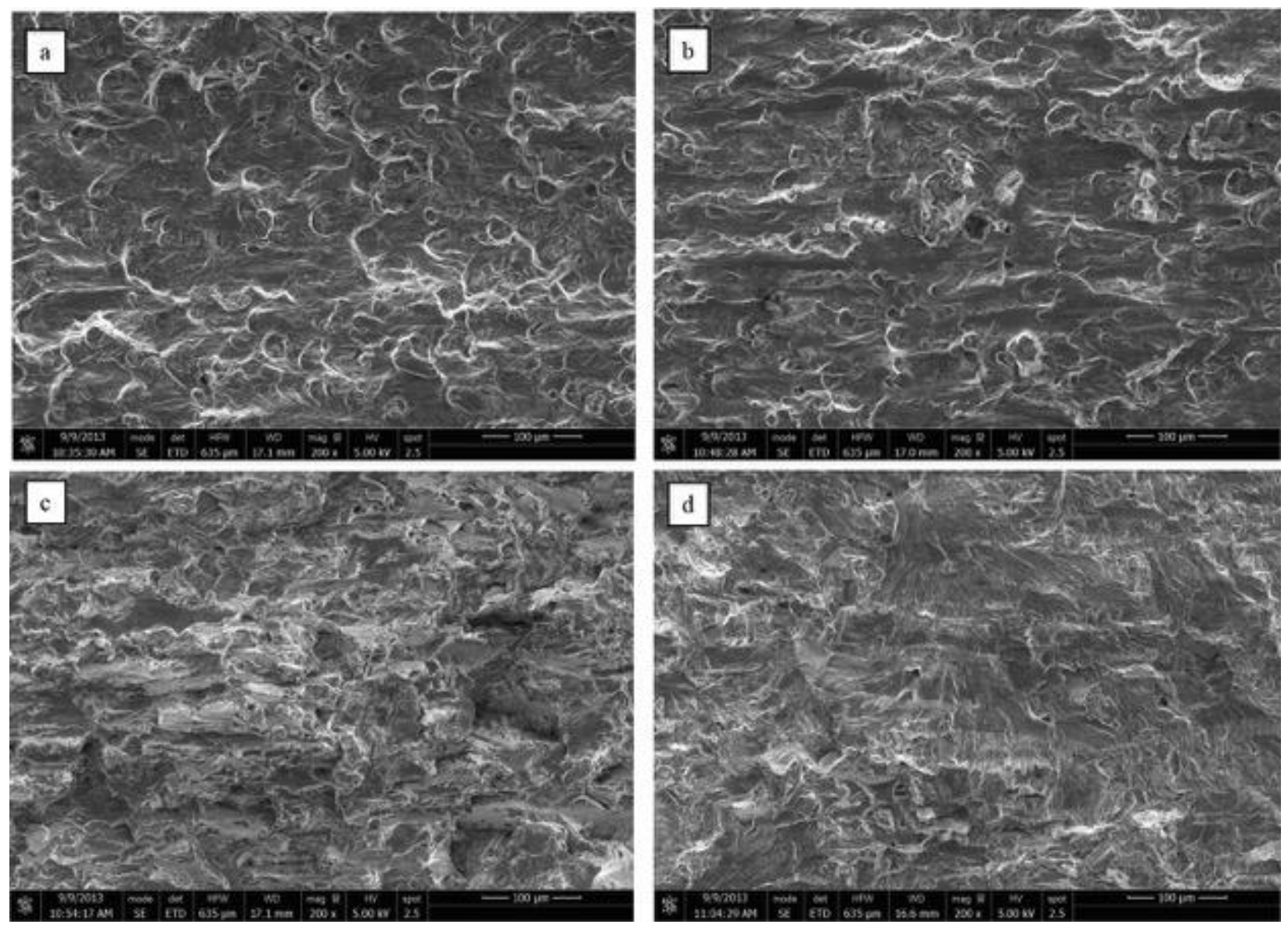

Figure 10: SEM images of the fatigue crack surface of 7075-T651 tested under decreasing $\Delta \mathrm{K}$ loading at constant $\mathrm{R}=0.5, f=20$ for an $\mathrm{L}-\mathrm{T}$ oriented specimen exposed to $\mathrm{P}_{\mathrm{H} 2 \mathrm{O}}=0.5 \mathrm{~Pa}$ with $\Delta \mathrm{K}$ of: (a) 7, (b) $\sim 6$, (c) $\sim 5$, and (d) $\sim 4.0 \mathrm{MPa} \sqrt{\mathrm{m}}$. The direction of crack growth is left to right in each image. 


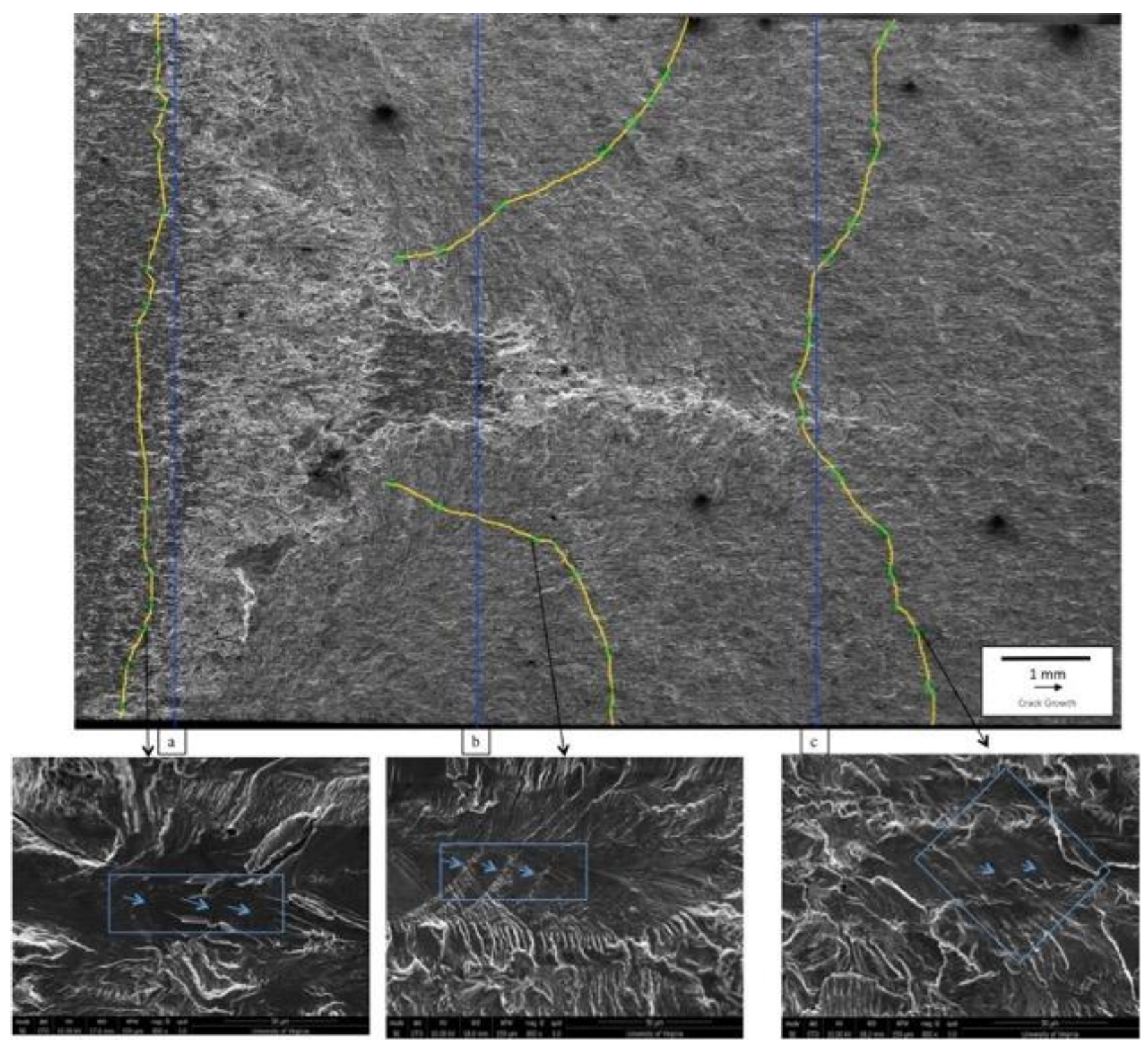

Figure 11: SEM overview of fracture surface produced from a Kshed loading protocol at a $\mathrm{P}_{\mathrm{H} 2 \mathrm{O}}$ $=0.5 \mathrm{~Pa}$ with vertical compliance calculated crack fronts (blue) and 3 experimental crack fronts identified by marker-bands (green/yellow lines). The green lines correspond to marker-bands identified in the fracture surface and the yellow lines are the inferred crack fronts used to connect the observed marker-bands. Example marker-bands produced at each $\Delta \mathrm{K}$ location are provided below the overview fracture surface for each $\Delta \mathrm{K}$ value. For constant $\mathrm{R}, \Delta \mathrm{K}$ decreased with increasing crack length from left to right in this image. The blue lines correspond to the compliance crack lengths associated with a $\Delta \mathrm{K}$ of: (a) 5, (b) 4, and (c) $3 \mathrm{MPa} \sqrt{\mathrm{m}}$. 


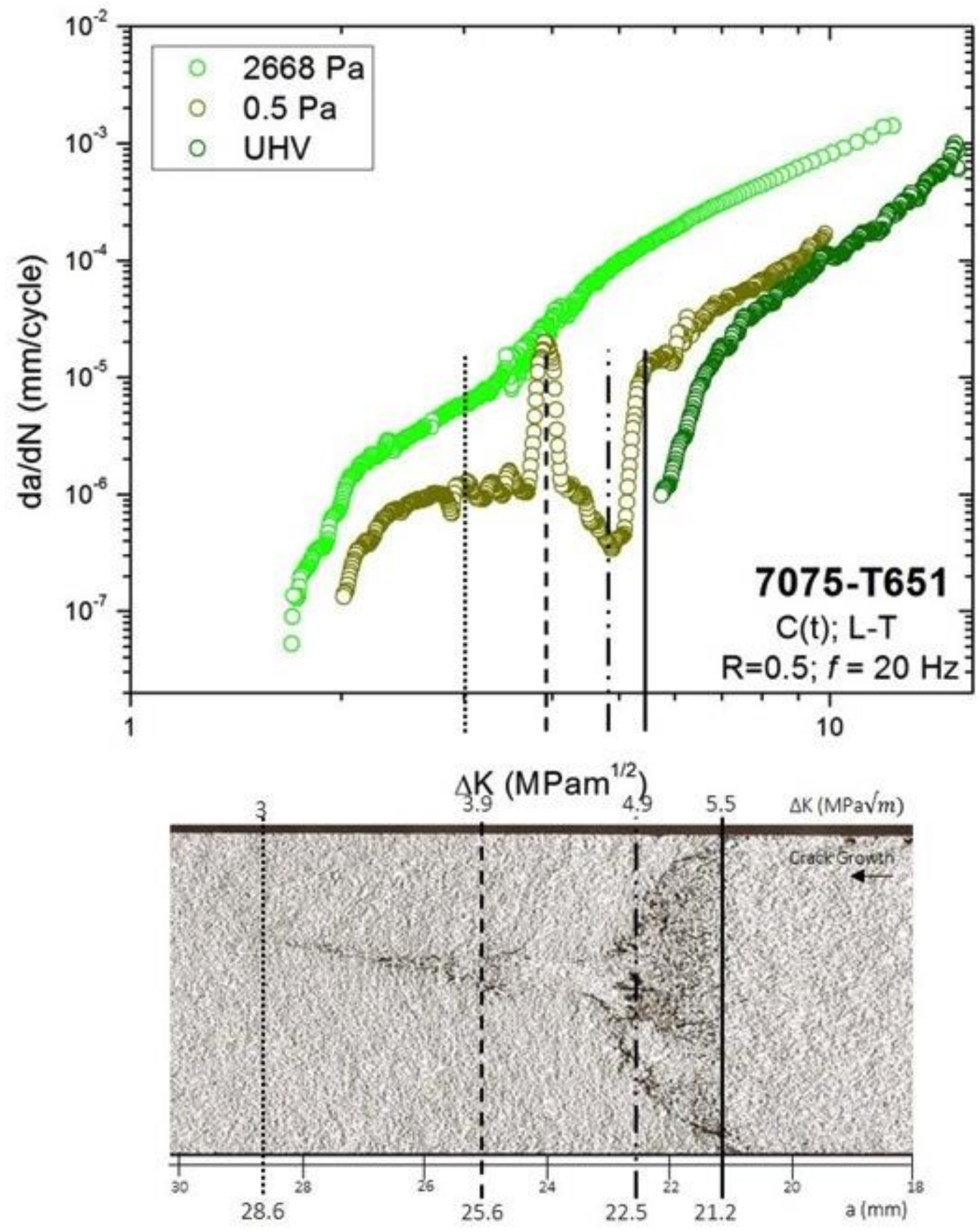

Figure 12: Crack growth rate versus $\Delta \mathrm{K}$ (a) and corresponding optical fractograph (b) illustrating the transition from macroscopically rough to smooth topography on the fatigue crack surface of 7075-T651 (L-T orientation) stressed in pure water vapor at $\mathrm{P}_{\mathrm{H} 2 \mathrm{O}}=0.5 \mathrm{~Pa}$. For constant $\mathrm{R}, \Delta \mathrm{K}$ decreased with increasing crack length from right to left in this image. The $0.5 \mathrm{~Pa}$ exposure showed a spike in da/dN approximately at $\Delta \mathrm{K}=3.9 \mathrm{MPa} \sqrt{\mathrm{m}}$. 

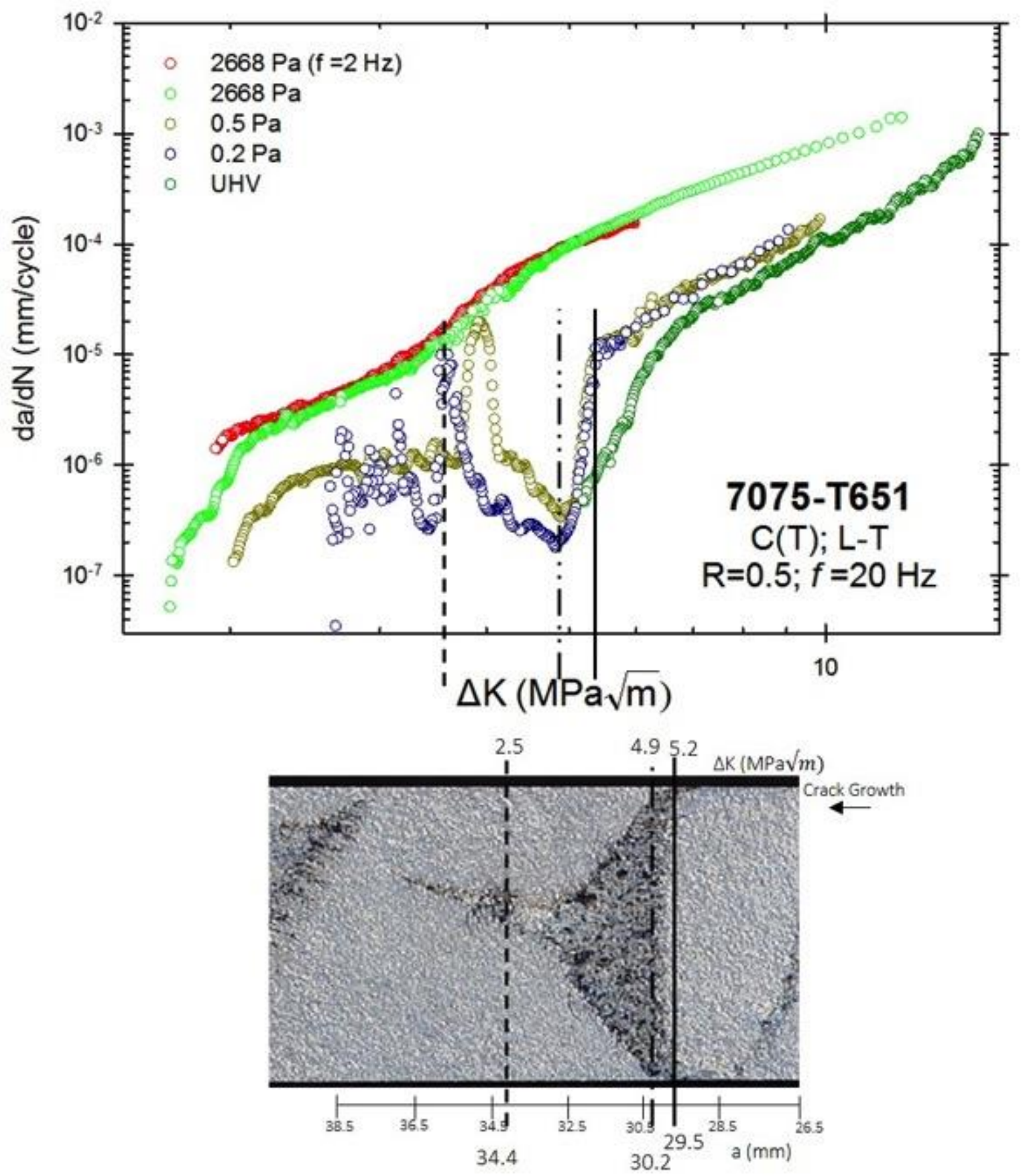

Figure 13: Crack growth rate versus $\Delta \mathrm{K}$ (a) and corresponding optical fractograph (b) illustrating the transition from macroscopically rough to smooth topography on the fatigue crack surface of 7075-T651 (L-T orientation) stressed in pure water vapor at $\mathrm{P}_{\mathrm{H} 2 \mathrm{O}}=0.2 \mathrm{~Pa}$. For constant $\mathrm{R}, \Delta \mathrm{K}$ decreased with increasing crack length from right to left in this image. The $0.2 \mathrm{~Pa}$ exposure showed a spike in da/dN approximately at $\Delta \mathrm{K}=3.6 \mathrm{MPa} \sqrt{\mathrm{m}}$. 

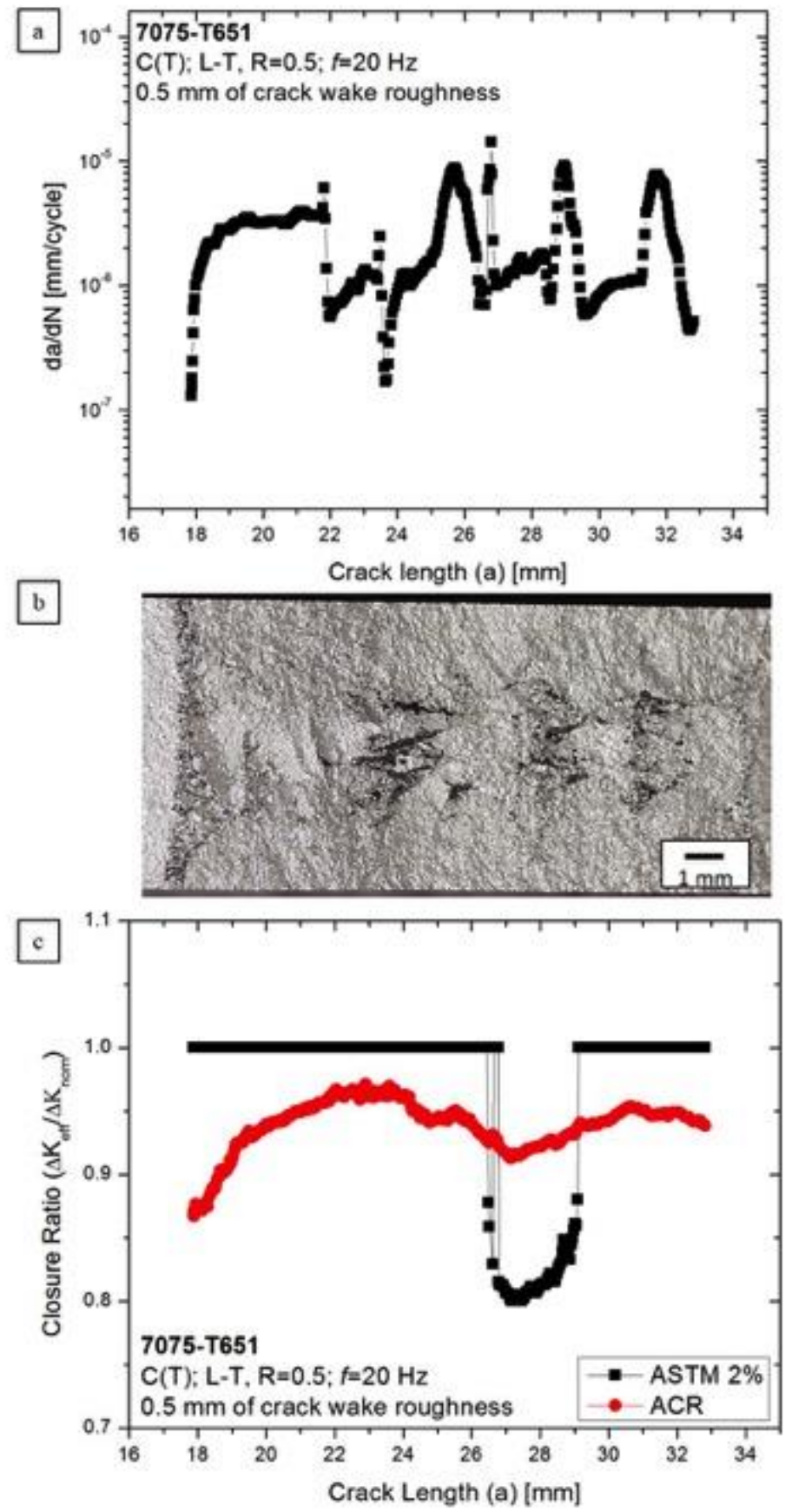

Figure 14: (a) Fatigue crack growth rate versus crack length at constant $\Delta \mathrm{K}=5 \mathrm{MPa} \sqrt{\mathrm{m}}$ and $\mathrm{R}$ of $0.50(f=20 \mathrm{~Hz})$ for $7075-\mathrm{T} 651(\mathrm{~L}-\mathrm{T})$ at $\mathrm{P}_{\mathrm{H} 2 \mathrm{O}}=0.5 \mathrm{~Pa} .0 .5 \mathrm{~mm}$ of crack wake roughness was imparted into the surface before beginning the exposure testing. (b) Optical fractograph of the test specimen corresponding to the crack length scale of the above and below plots. Crack growth occurred left to right. (c) Closure analysis (ASTM 2\% and ACR ratio) versus crack length. 

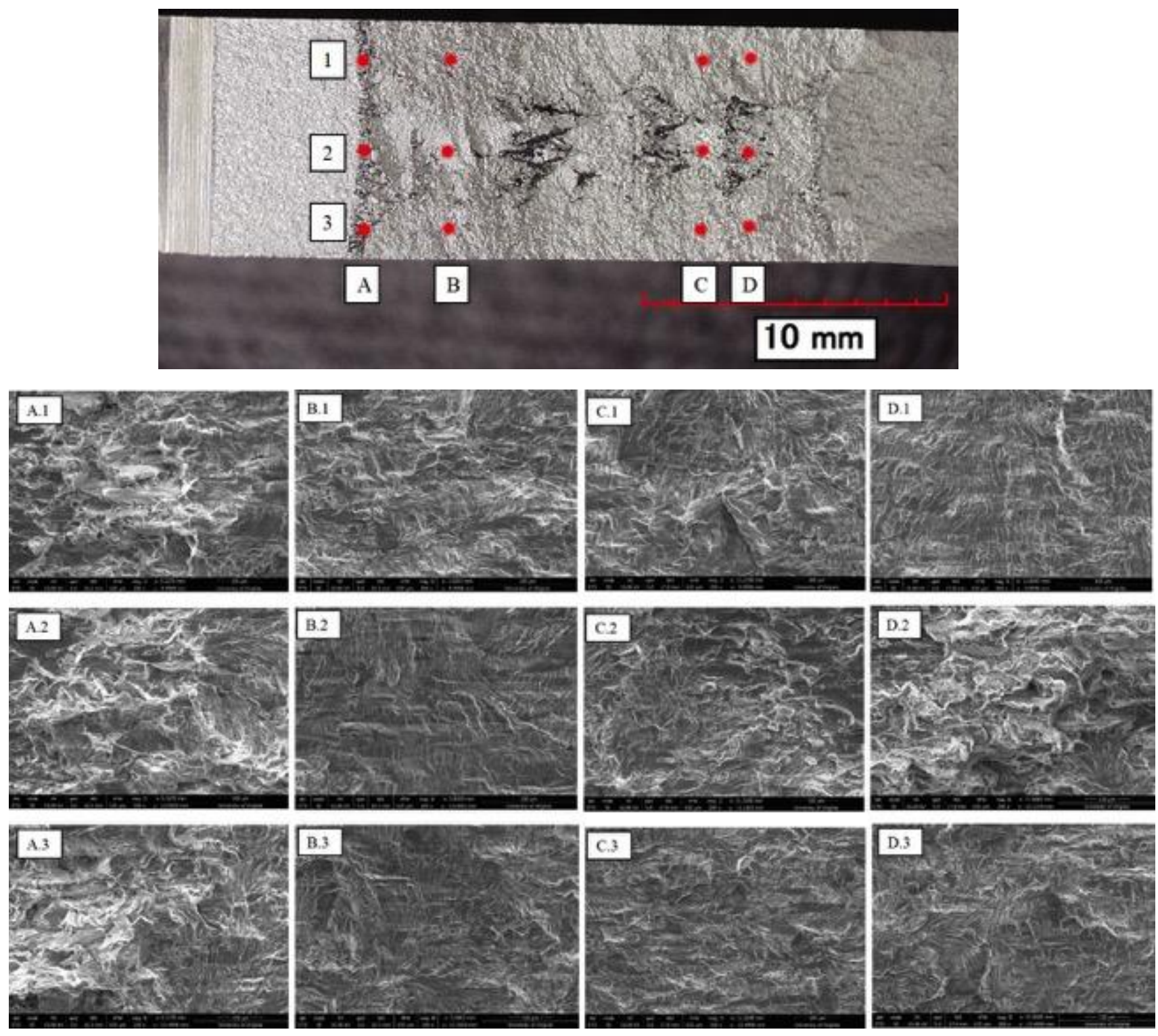

Figure 15: Optical fractograph of the $0.5 \mathrm{~mm}$ of crack wake roughness test specimen tested at $\mathrm{P}_{\mathrm{H} 2 \mathrm{O}}$ $=0.5 \mathrm{~Pa}$. The SEM images below correspond to the number location where the red dots are on the optical fracture surface. Crack growth occurred left to right. 

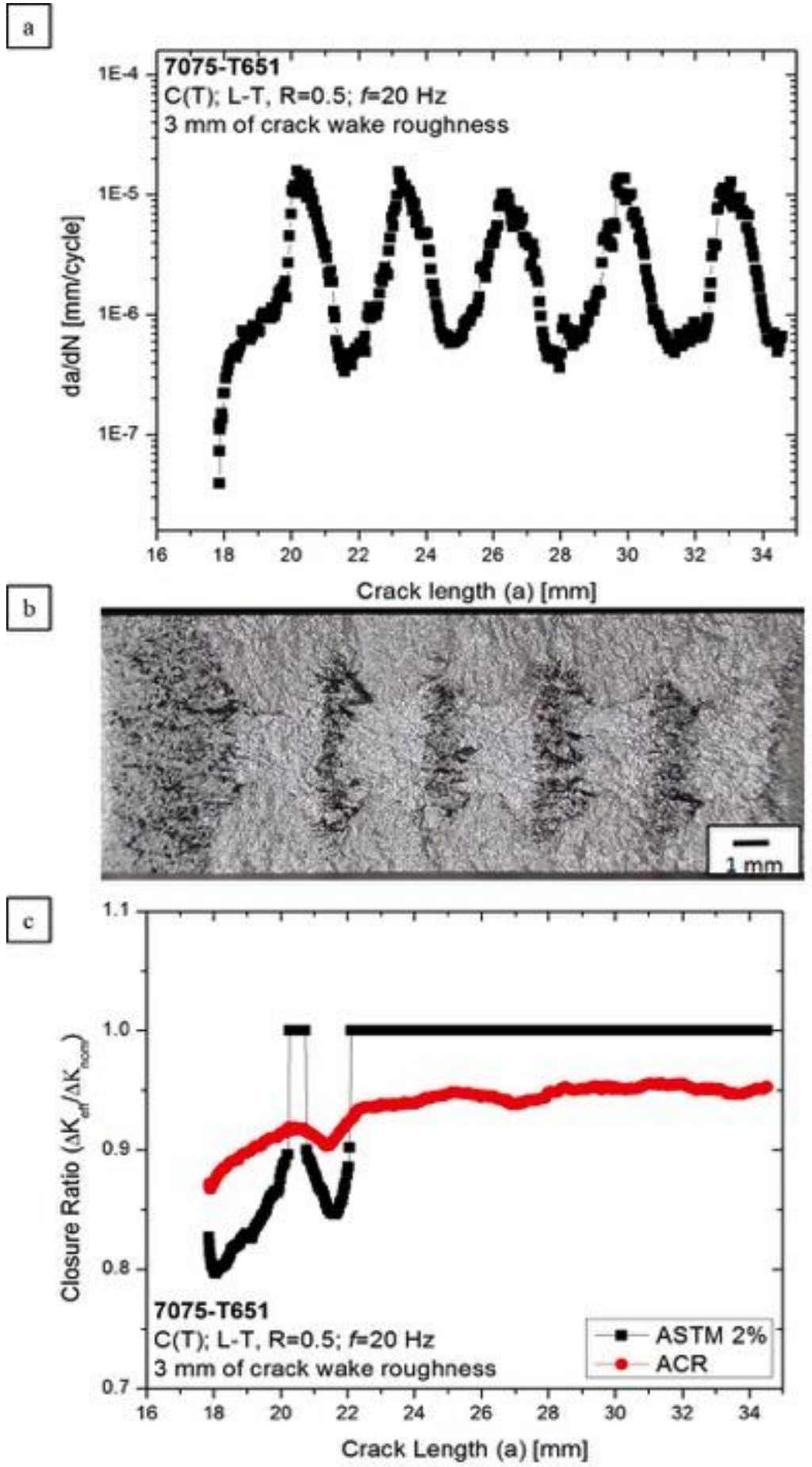

Figure 16: (a) Fatigue crack growth rate versus crack length at constant $\Delta \mathrm{K}=5 \mathrm{MPa} \sqrt{\mathrm{m}}$ and $\mathrm{R}$ of $0.50(f=20 \mathrm{~Hz})$ for $7075-\mathrm{T} 651(\mathrm{~L}-\mathrm{T})$ at $\mathrm{P}_{\mathrm{H} 2 \mathrm{O}}=0.5 \mathrm{~Pa} .3 \mathrm{~mm}$ of crack wake roughness was imparted into the surface before beginning the exposure testing. (b) Optical fractograph of the test specimen corresponding to the crack length scale of the above and below plots. Crack growth occurred left to right. (c) Closure analysis (ASTM 2\% and ACR ratio) versus crack length. 

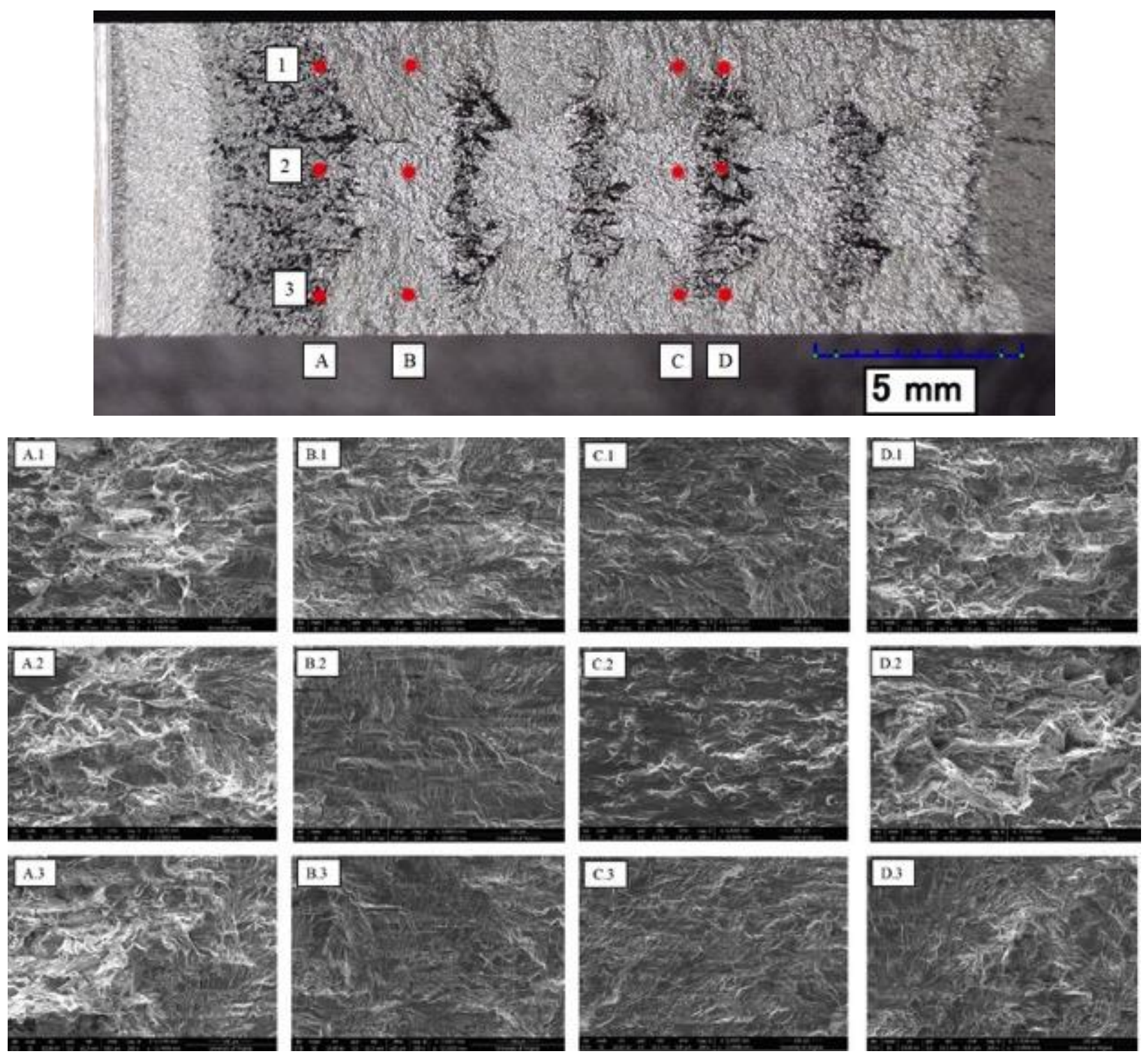

Figure 17: Optical fractograph of the $3 \mathrm{~mm}$ of crack wake roughness test specimen tested at $\mathrm{P}_{\mathrm{H} 2 \mathrm{O}}$ $=0.5 \mathrm{~Pa}$. The SEM images below correspond to the number location where the red dots are on the optical fracture surface. Crack growth occurred left to right. 


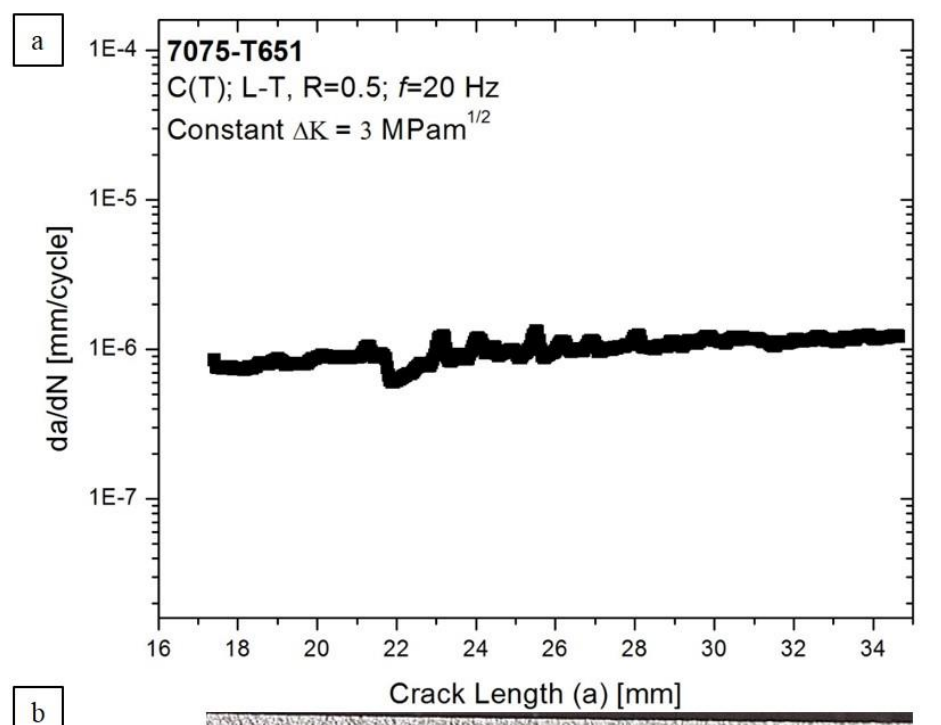

$\mathrm{b}$
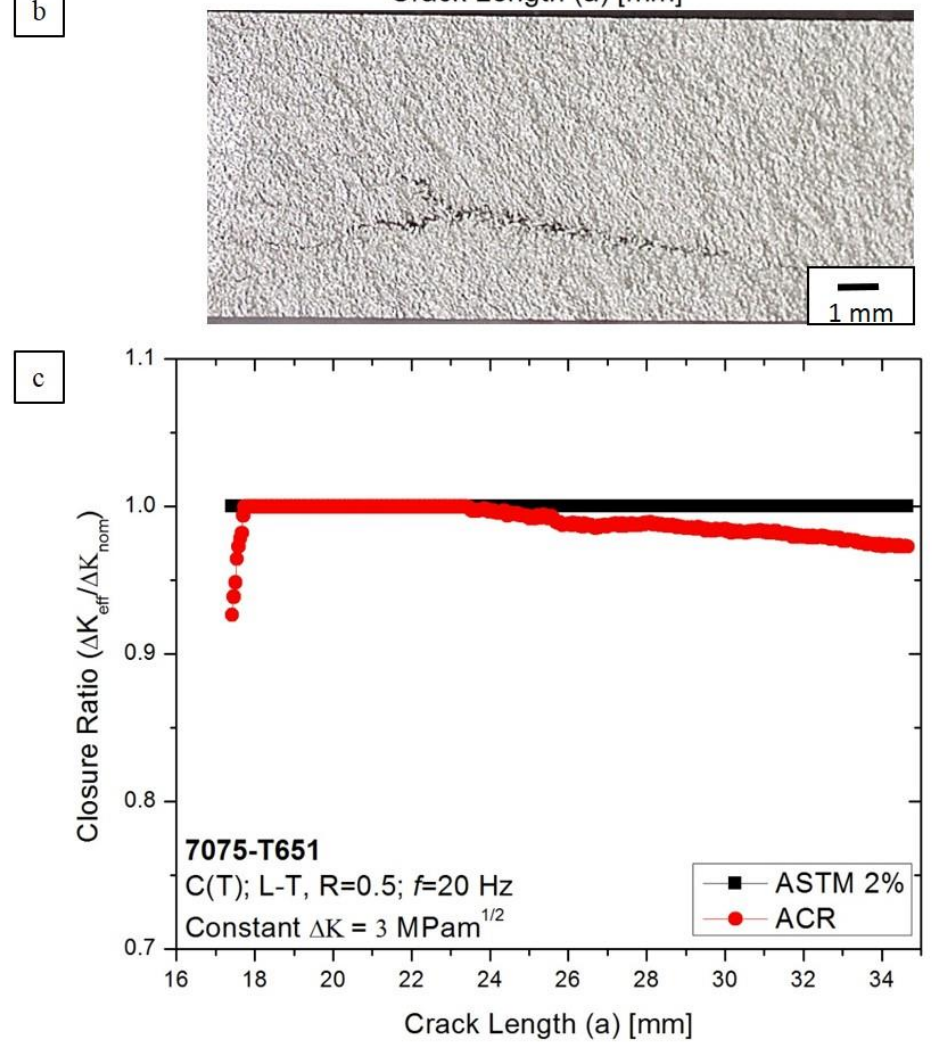

Figure 18: (a) Fatigue crack growth rate versus crack length at constant $\Delta \mathrm{K}=3 \mathrm{MPa} \sqrt{\mathrm{m}}$ and $\mathrm{R}$ of $0.50(f=20 \mathrm{~Hz})$ for $7075-\mathrm{T} 651(\mathrm{~L}-\mathrm{T})$ at $\mathrm{P}_{\mathrm{H} 2 \mathrm{O}}=0.5 \mathrm{~Pa}$. No crack rack roughness or load effects were imparted into the surface before beginning the exposure testing. (b) Optical fractograph of the test specimen corresponding to the crack length scale of the above and below plots. Crack growth occurred left to right. (c) Closure analysis (ASTM 2\% and ACR ratio) versus crack length. 
a

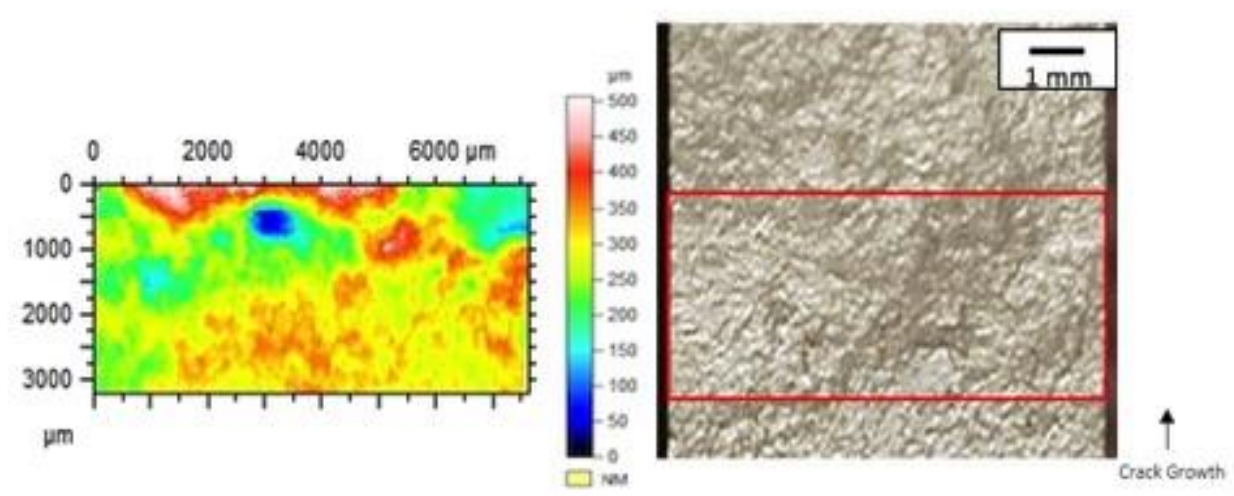

b

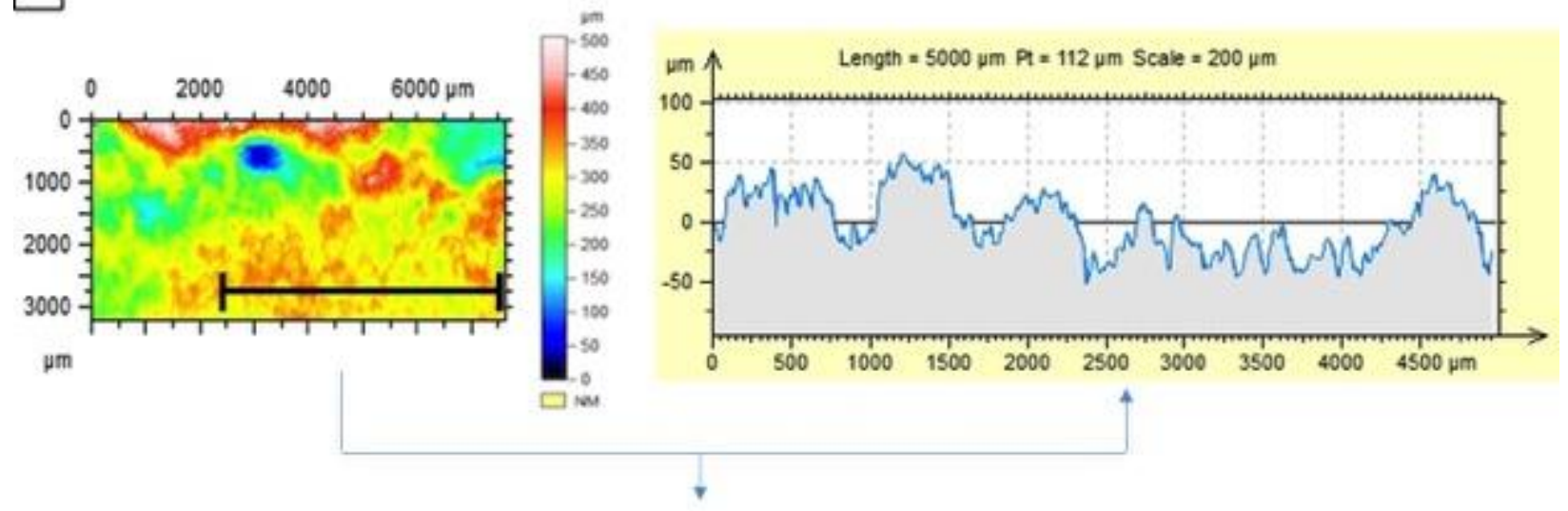

\begin{tabular}{|c|c|}
\hline \multicolumn{2}{|c|}{ Amplitude Parameters } \\
\hline$R a$ & $10.2 \mu \mathrm{m}$ \\
\hline$R q$ & $12.2 \mu \mathrm{m}$ \\
\hline$R v$ & $26.6 \mu \mathrm{m}$ \\
\hline$R p$ & $23.2 \mu \mathrm{m}$ \\
\hline
\end{tabular}

Figure 19: (a) 3D characterization and the corresponding optical fractograph of the threshold transition regime area for the 7075-T651 1.8 Pa sample produced under decreasing $\Delta \mathrm{K}$ loading at constant $\mathrm{R}$ of $0.50(f=20 \mathrm{~Hz})$, (b) 3D characterization of the $1.8 \mathrm{~Pa}$ sample with a horizontal line scan (shown by the black line). The plot on the right graphs the localized topography of the crack face corresponding to the horizontal line segment. From this plot, the local roughness parameters ( $\mathrm{Ra}$ - average roughness, $\mathrm{Rq}$ - geometric average roughness, $\mathrm{Rp}$ - average peak height, Rvaverage valley height) can be gleaned for the horizontal line segment of interest. 
a
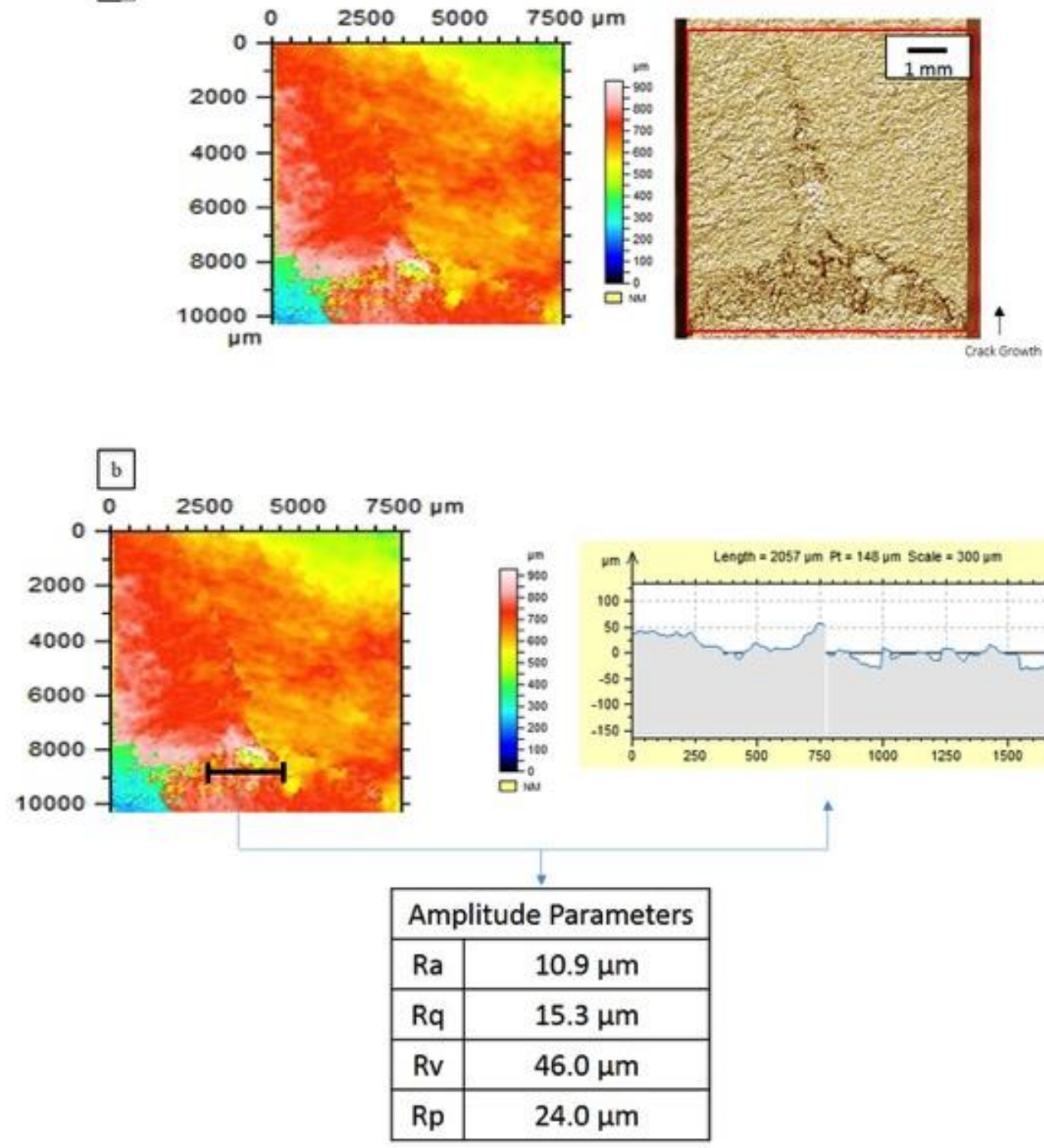

Figure 20: (a) 3D characterization and the corresponding optical fractograph of the threshold transition regime area for the 7075-T651 0.5 Pa sample produced under decreasing $\Delta \mathrm{K}$ loading at constant $\mathrm{R}$ of $0.50(f=20 \mathrm{~Hz})$, (b) 3D characterization of the $0.5 \mathrm{~Pa}$ sample with a horizontal line scan (shown by the black line). The plot on the right graphs the localized topography of the crack face corresponding to the horizontal line segment. From this plot, the local roughness parameters ( $\mathrm{Ra}$ - average roughness, $\mathrm{Rq}$ - geometric average roughness, $\mathrm{Rp}$ - average peak height, Rvaverage valley height) can be gleaned for the horizontal line segment of interest. 
a

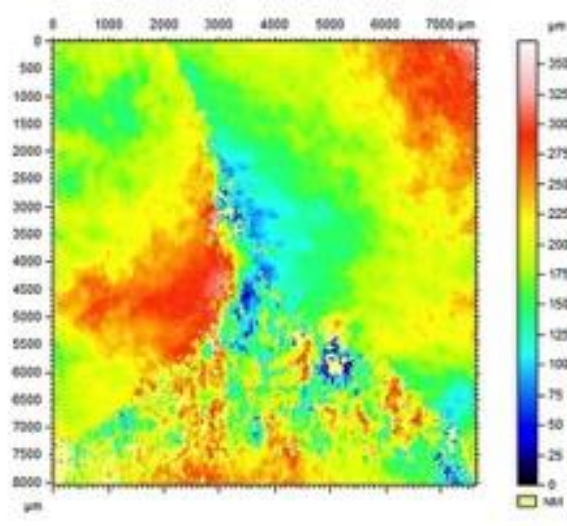

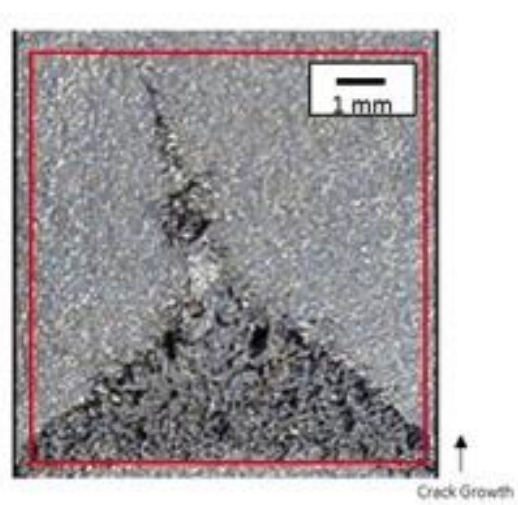

$\mathrm{b}$

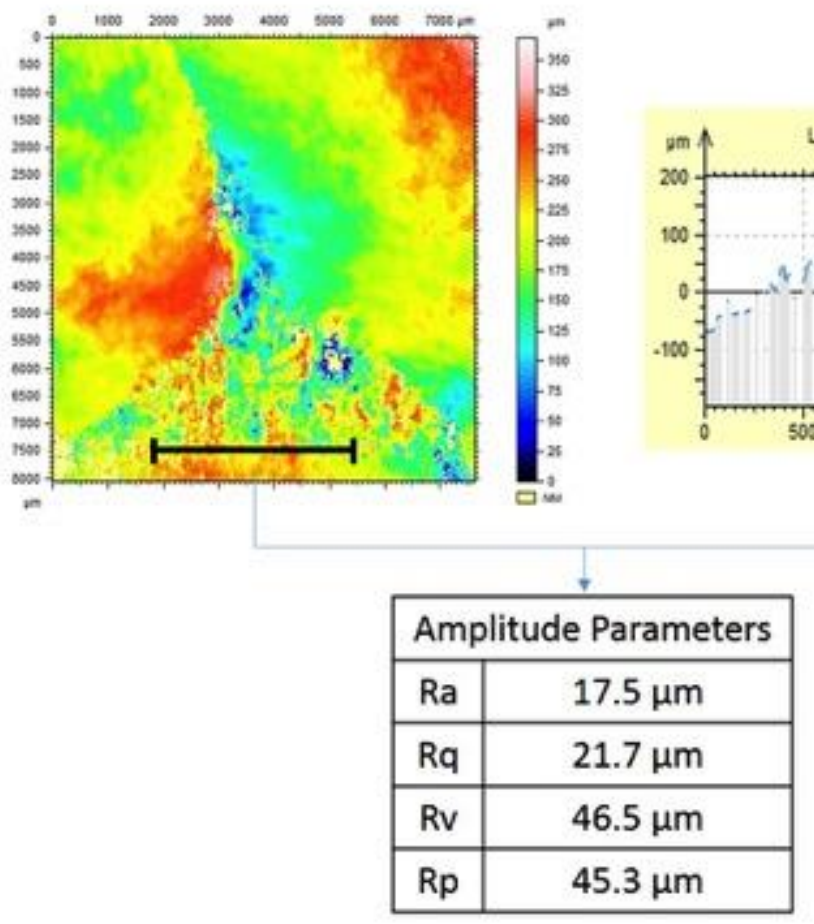

Figure 21: (a) 3D characterization and the corresponding optical fractograph of the threshold transition regime area for the 7075-T651 0.2 Pa sample produced under decreasing $\Delta \mathrm{K}$ loading at constant $\mathrm{R}$ of $0.50(f=20 \mathrm{~Hz})$, (b) 3D characterization of the $0.2 \mathrm{~Pa}$ sample with a horizontal line scan (shown by the black line). The plot on the right graphs the localized topography of the crack face corresponding to the horizontal line segment. From this plot, the local roughness parameters ( $\mathrm{Ra}$ - average roughness, $\mathrm{Rq}$ - geometric average roughness, Rp - average peak height, Rvaverage valley height) can be gleaned for the horizontal line segment of interest. 


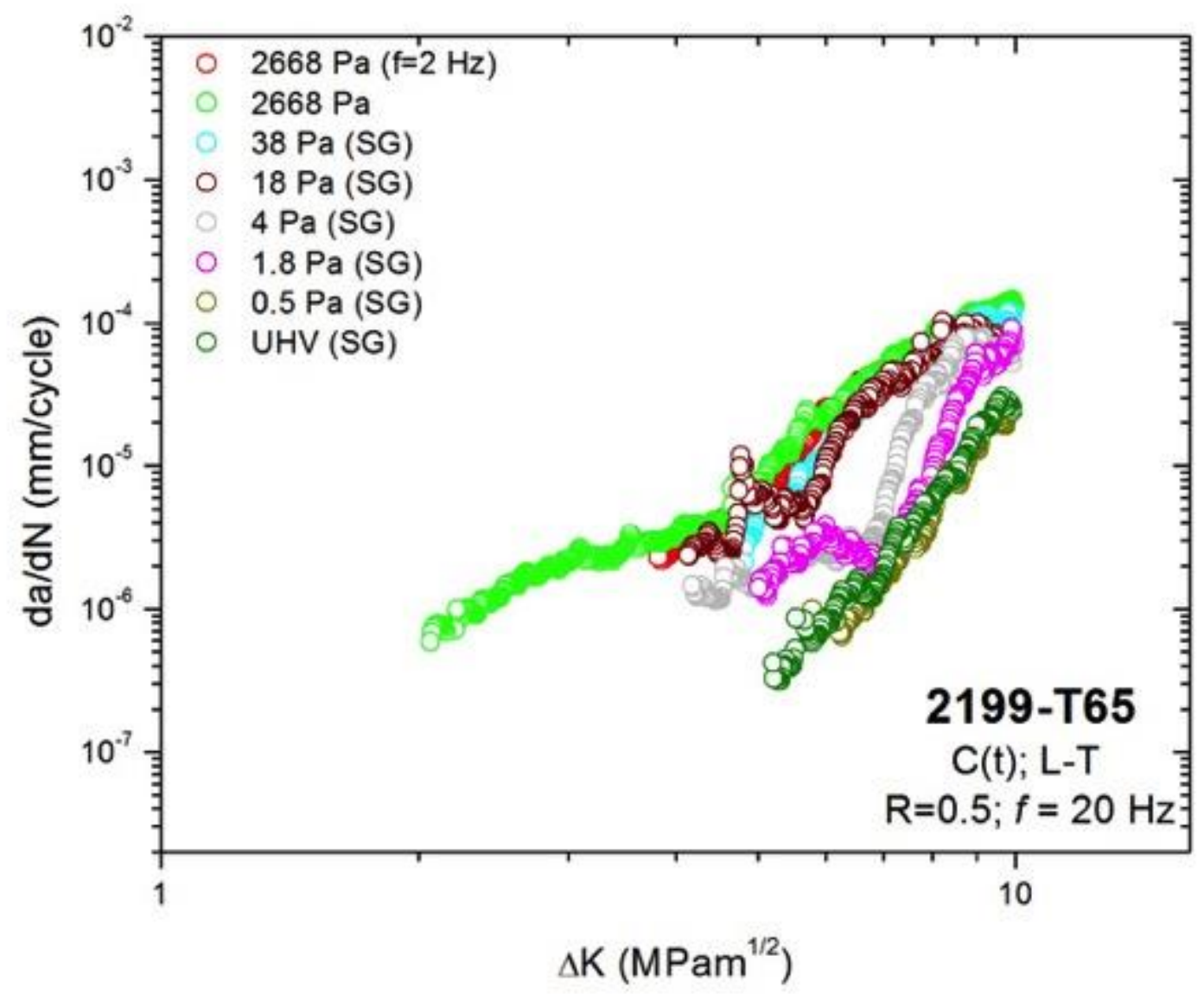

Figure 22: $\quad$ Fatigue crack growth rate versus decreasing $\Delta \mathrm{K}$ at constant $\mathrm{R}$ of $0.50(f=20 \mathrm{~Hz})$ for 2199-T86 (L-T) at various-constant water vapor exposure levels from ultra-high vacuum $(0.25$ $0.50 \mu \mathrm{Pa}-\mathrm{s})$ to $1.3 \mathrm{kPa}-\mathrm{s}\left(\mathrm{P}_{\mathrm{H} 2 \mathrm{O}}=26.7 \mathrm{kPa}\right)$. 

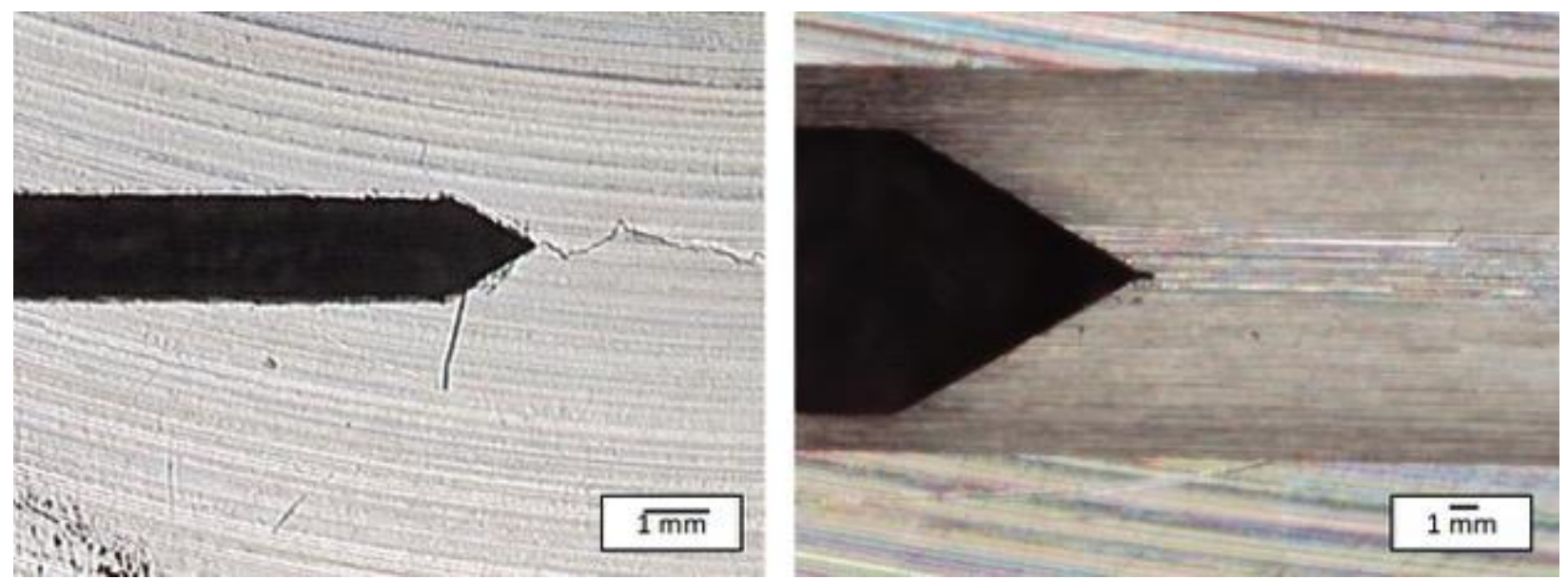

Figure 23: Optical image of the notch for the 2199-T86 un-grooved (left) and side-grooved (right) C-T specimens. Additionally, a small notch was added to the preexisting CT notch through electrical discharge machining (EDM) for the side-grooved specimens. The side-grooving process reduced the overall thickness of the specimen in the crack plane. 


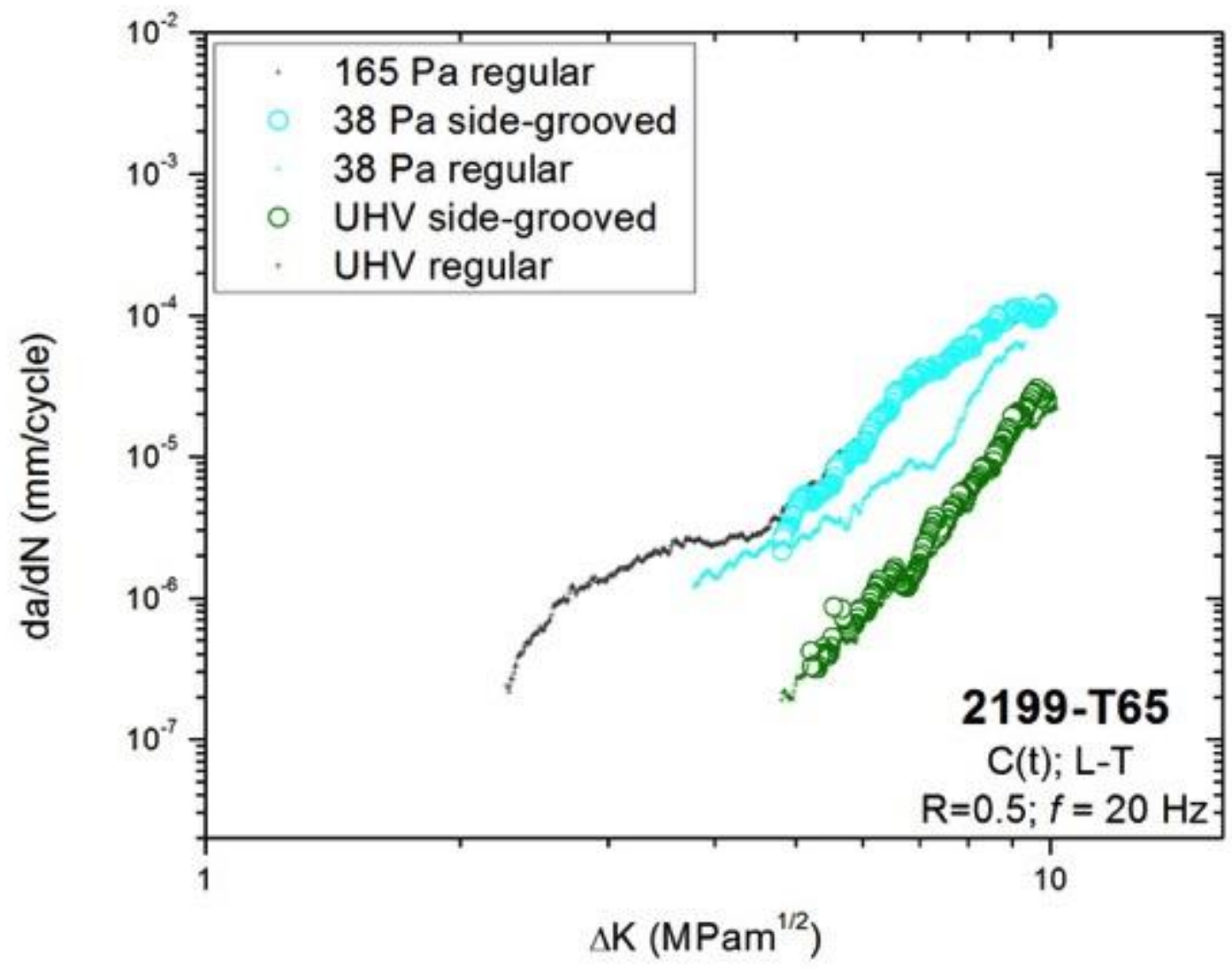

Figure 24: Fatigue crack growth rate versus decreasing $\Delta \mathrm{K}$ comparison for the 2199-T86 sidegrooved and un-grooved C-T specimens at constant $\mathrm{R}$ of $0.50(f=20 \mathrm{~Hz})$ for three constant water vapor exposure levels from ultra-high vacuum $(0.25-0.50 \mu \mathrm{Pa}-\mathrm{s})$ to $165 \mathrm{~Pa}$. The growth rate data for the side-grooved specimens are open circles and the un-grooved specimens are labeled as "regular" and are shown as crosses. 


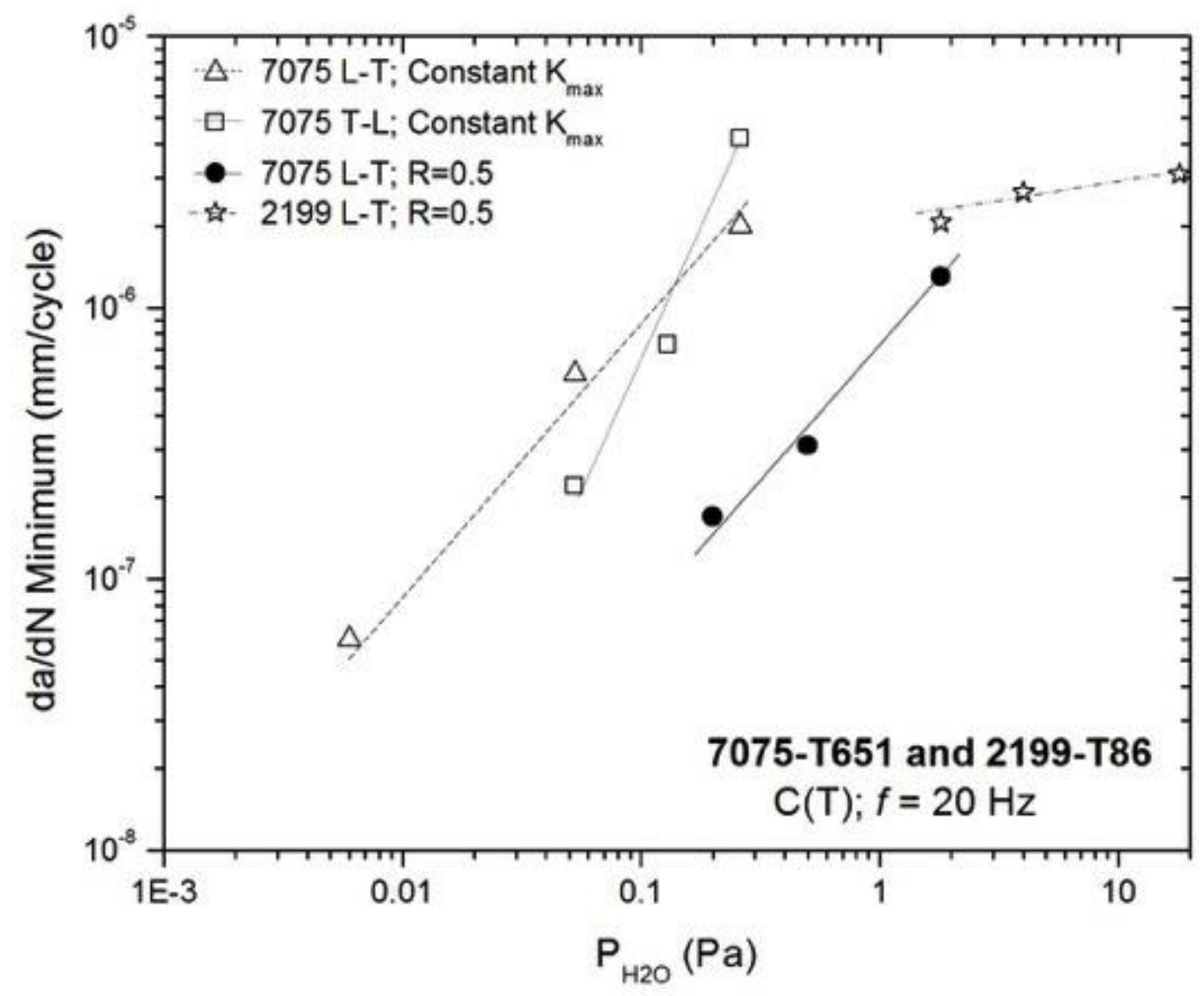

Figure 25: Effect of water vapor exposure on the observed crack growth rate minima in the threshold transition regime for 7075-T651 loaded under constant $K_{\max }(16.5 \mathrm{MPa} \sqrt{ }$ ) -decreasing $\Delta \mathrm{K}$ in both $\mathrm{L}-\mathrm{T}$ and $\mathrm{T}$-L orientations as well as constant $\Delta \mathrm{K}$-constant $\mathrm{R}(0.5)$ loading in the $\mathrm{L}-\mathrm{T}$ orientation (Figure 1) as shown in previous work [1]. Additionally, the effect of water vapor exposure on the observed crack growth rate minima in the threshold transition regime for 2199T86 loaded under decreasing $\Delta \mathrm{K}$ in the L-T orientation (Figure 22) is plotted. 

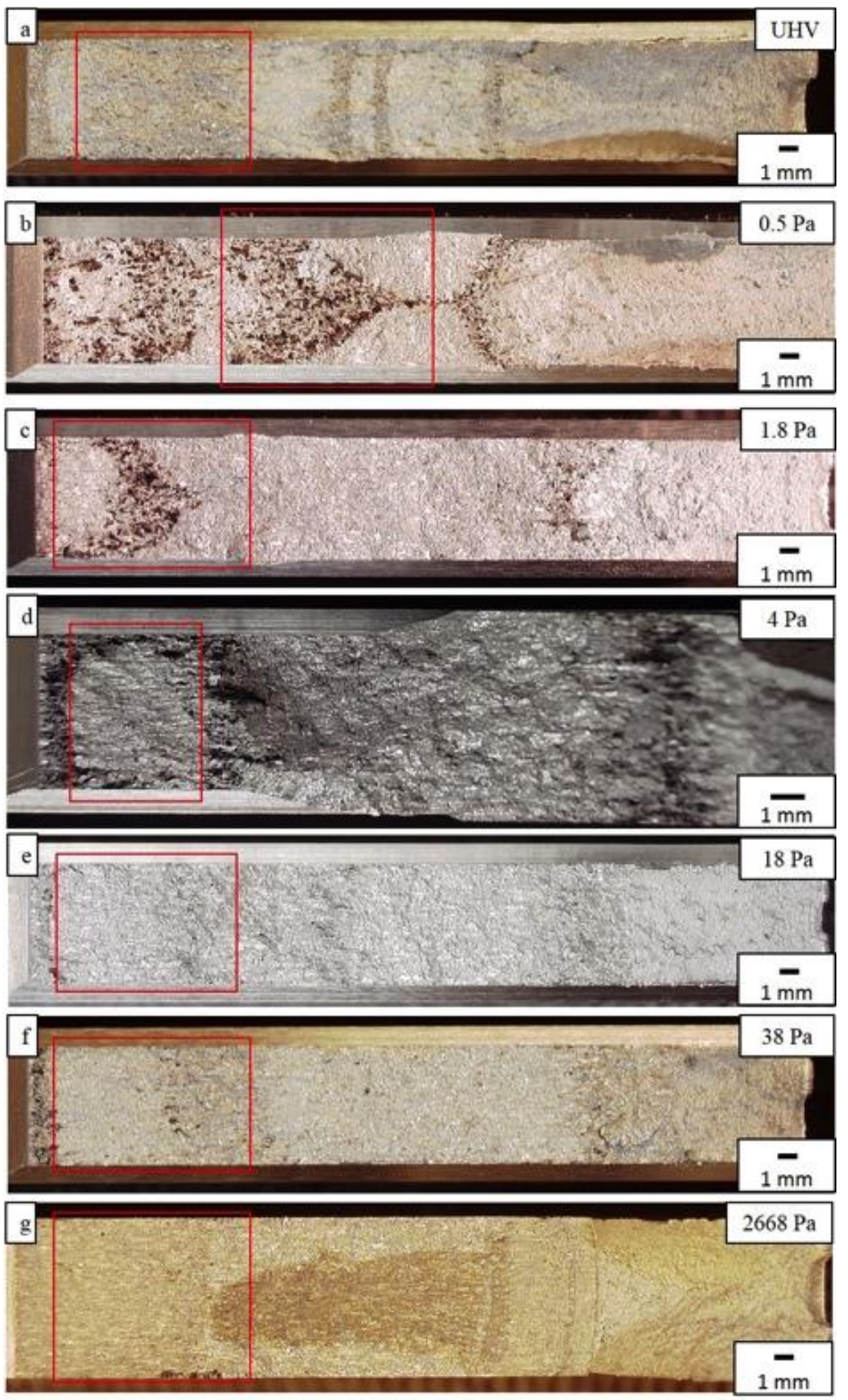

Figure 26: Optical fractographs for decreasing $\Delta \mathrm{K}$ at constant $\mathrm{R}$ of $0.50(f=20 \mathrm{~Hz})$ for 2199-T86 (L-T) at various-constant water vapor exposure levels: (a) Ultra-high vacuum, (b) 0.5, (c) 1.8, (d) 4, (e) 18, (f) 38, and (g) $2668 \mathrm{~Pa}$. Crack growth is left to right in each image. 

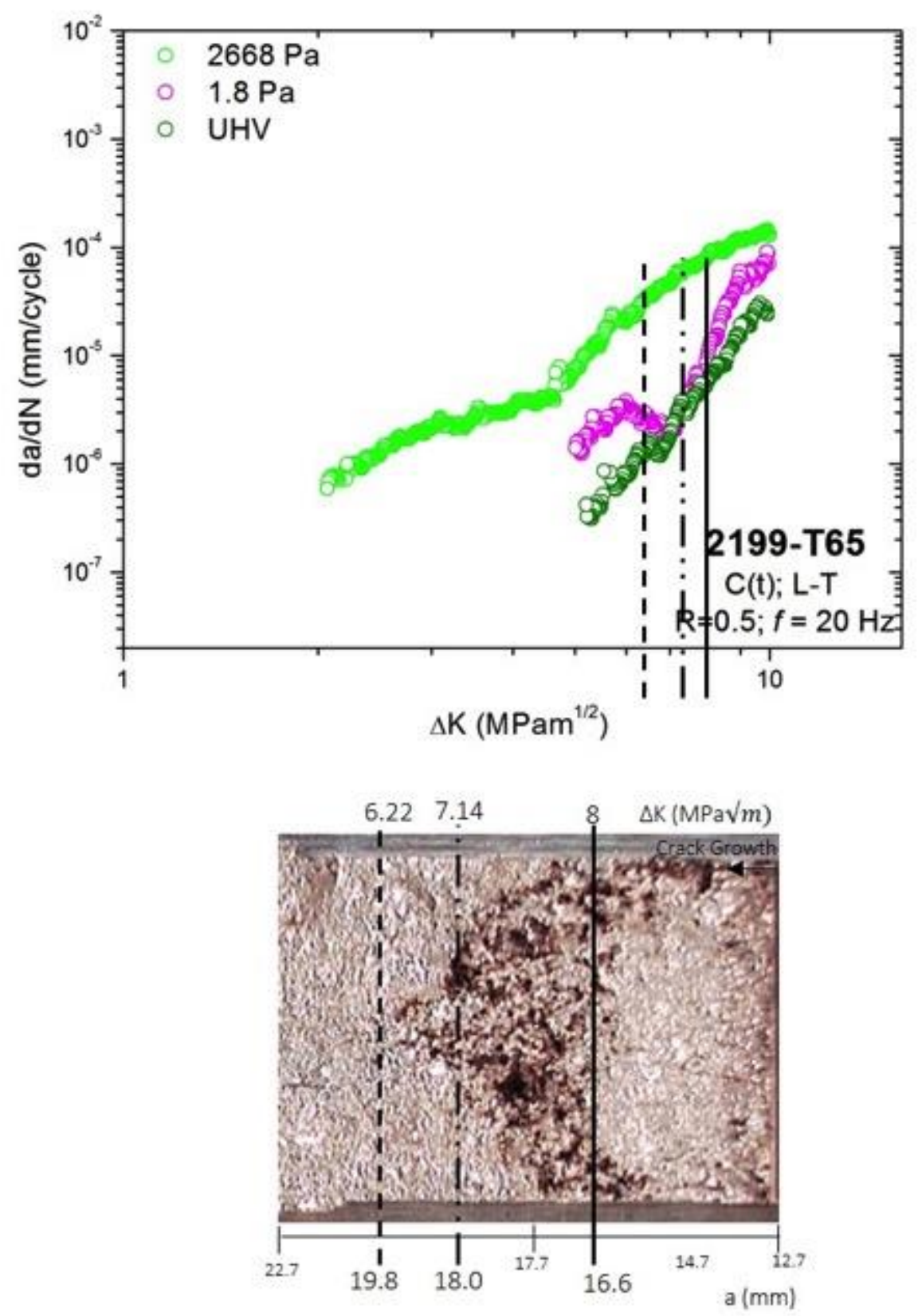

Figure 27: Crack growth rate versus $\Delta \mathrm{K}$ (a) and corresponding optical fractograph (b) illustrating the transition from macroscopically smooth to rough topography on the fatigue crack surface of 2199-T86 (L-T orientation) stressed in pure water vapor at $\mathrm{P}_{\mathrm{H} 2 \mathrm{O}}=1.8 \mathrm{~Pa}$. For constant $\mathrm{R}, \Delta \mathrm{K}$ decreased with increasing crack length from right to left in this image. 

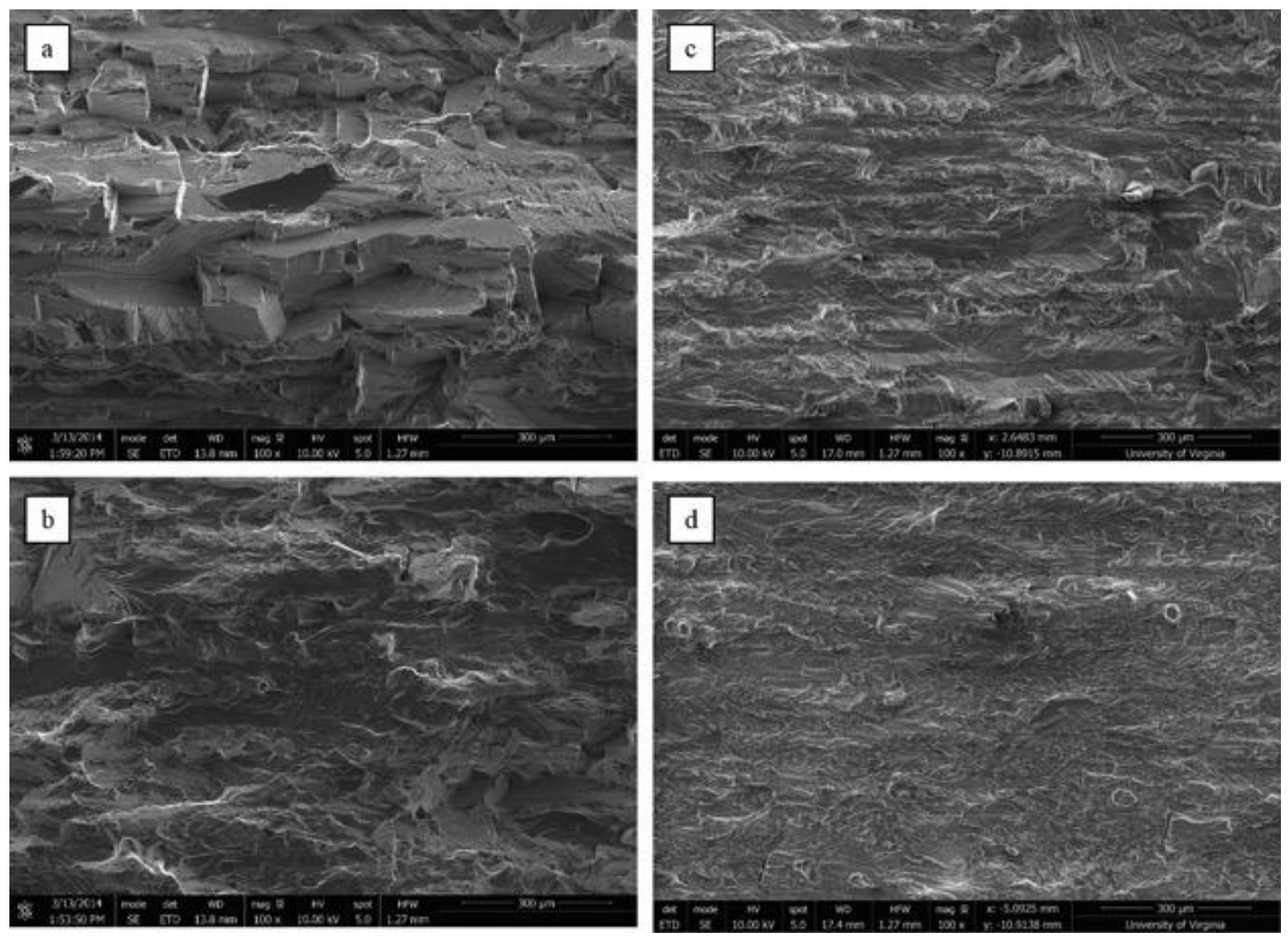

Figure 28: $\quad$ SEM images of the fatigue crack surface of 2199-T86 tested at constant $\mathrm{R}=0.5, f=$ 20 (from Figure 22) for an L-T oriented specimen exposed to $\mathrm{P}_{\mathrm{H} 2 \mathrm{O}}$ of: UHV (a-b) and high humidity $(2668 \mathrm{~Pa})(\mathrm{c}-\mathrm{d})$ with a $\Delta \mathrm{K}$ of: $(\mathrm{a}, \mathrm{c}) \sim 5,(\mathrm{~b}, \mathrm{~d}) \sim 9 \mathrm{MPa} \sqrt{\mathrm{m}}$. Crack growth is from left to right in each image. 

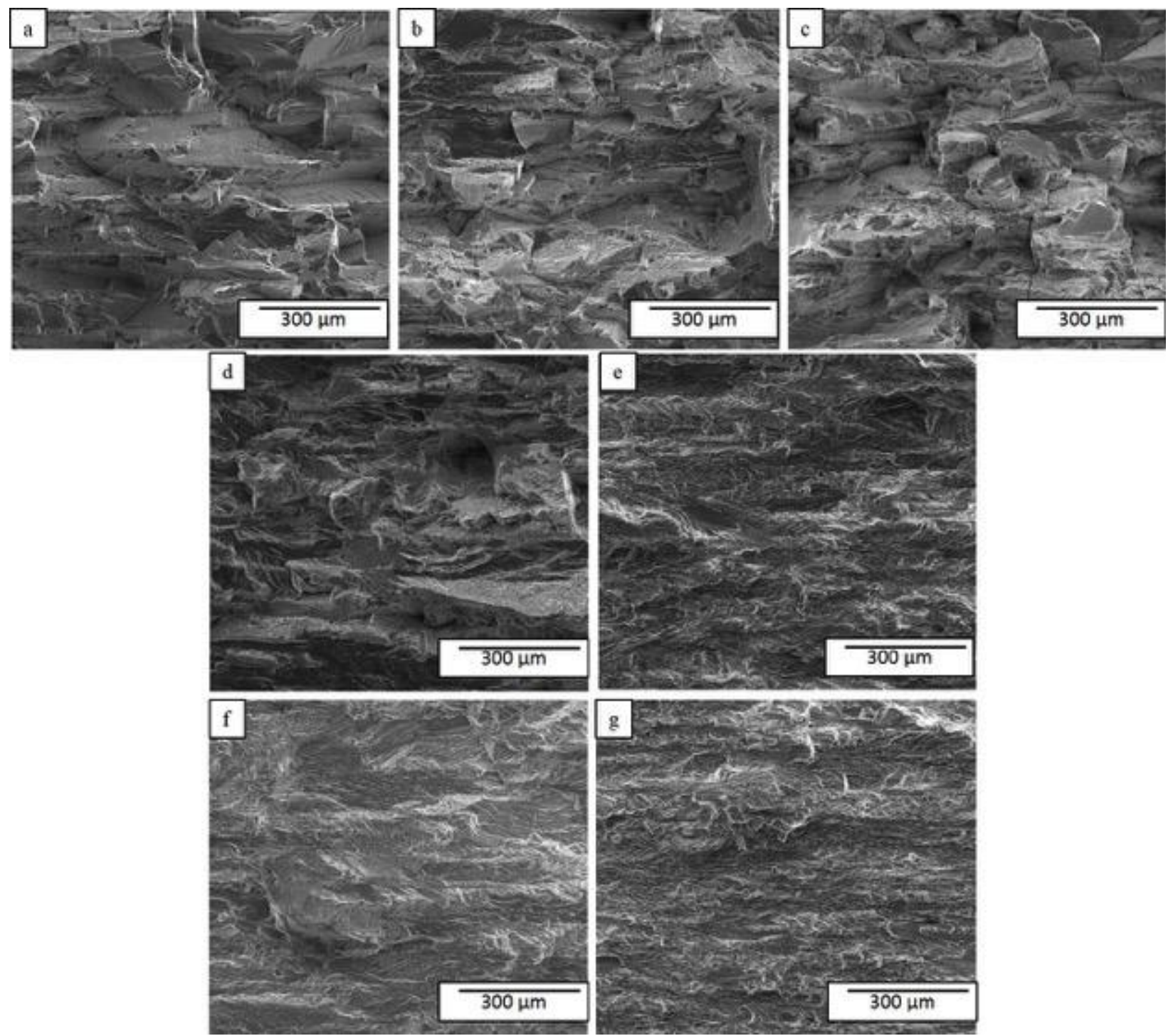

Figure 29: SEM images of the fatigue crack surface of 2199-T86 tested at constant $\mathrm{R}=0.5, f=$ $20 \mathrm{~Hz}$ and constant $\Delta \mathrm{K}=7 \mathrm{MPa} \sqrt{\mathrm{m}}$ (from Figure 22) for an L-T oriented specimen exposed to $\mathrm{P}_{\mathrm{H} 2 \mathrm{O}}$ of: (a) UHV (b) 0.5, (c) 1.8, (d) 4, (e) 18, (f) 38, and (g) $2668 \mathrm{~Pa}$. Crack growth is from left to right in each image. 

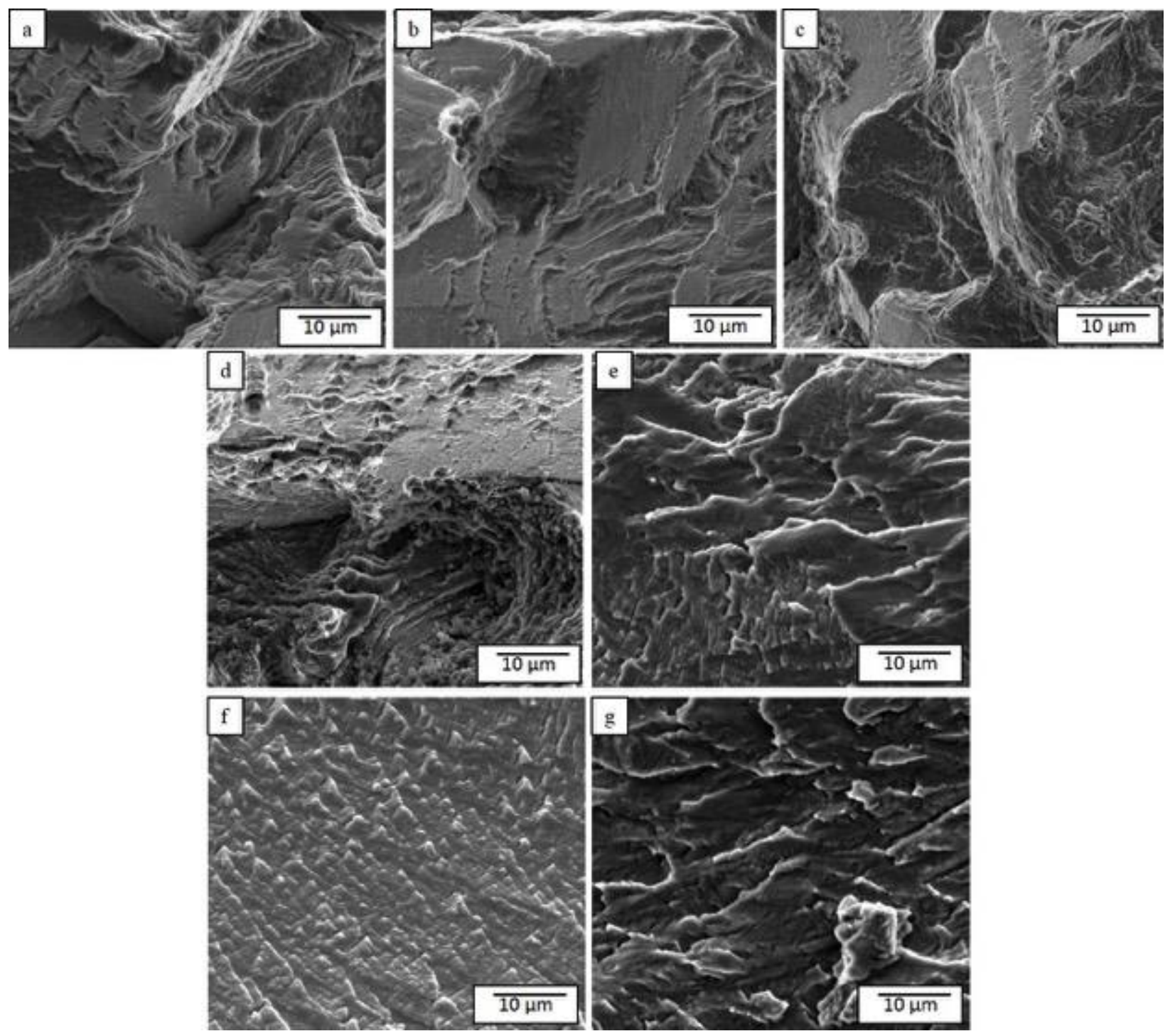

Figure 30: Higher magnification SEM images of the fatigue crack surface of 2199-T86 tested at constant $\mathrm{R}=0.5, f=20 \mathrm{~Hz}$ and constant $\Delta \mathrm{K}=7 \mathrm{MPa} \sqrt{\mathrm{m}}$ (from Figure 22) for an L-T oriented specimen exposed to $\mathrm{P}_{\mathrm{H} 2 \mathrm{O}}$ of: (a) UHV (b) 0.5, (c) 1.8, (d) 4, (e) 18, (f) 38, and (g) $2668 \mathrm{~Pa}$. Crack growth is from left to right in each image. 

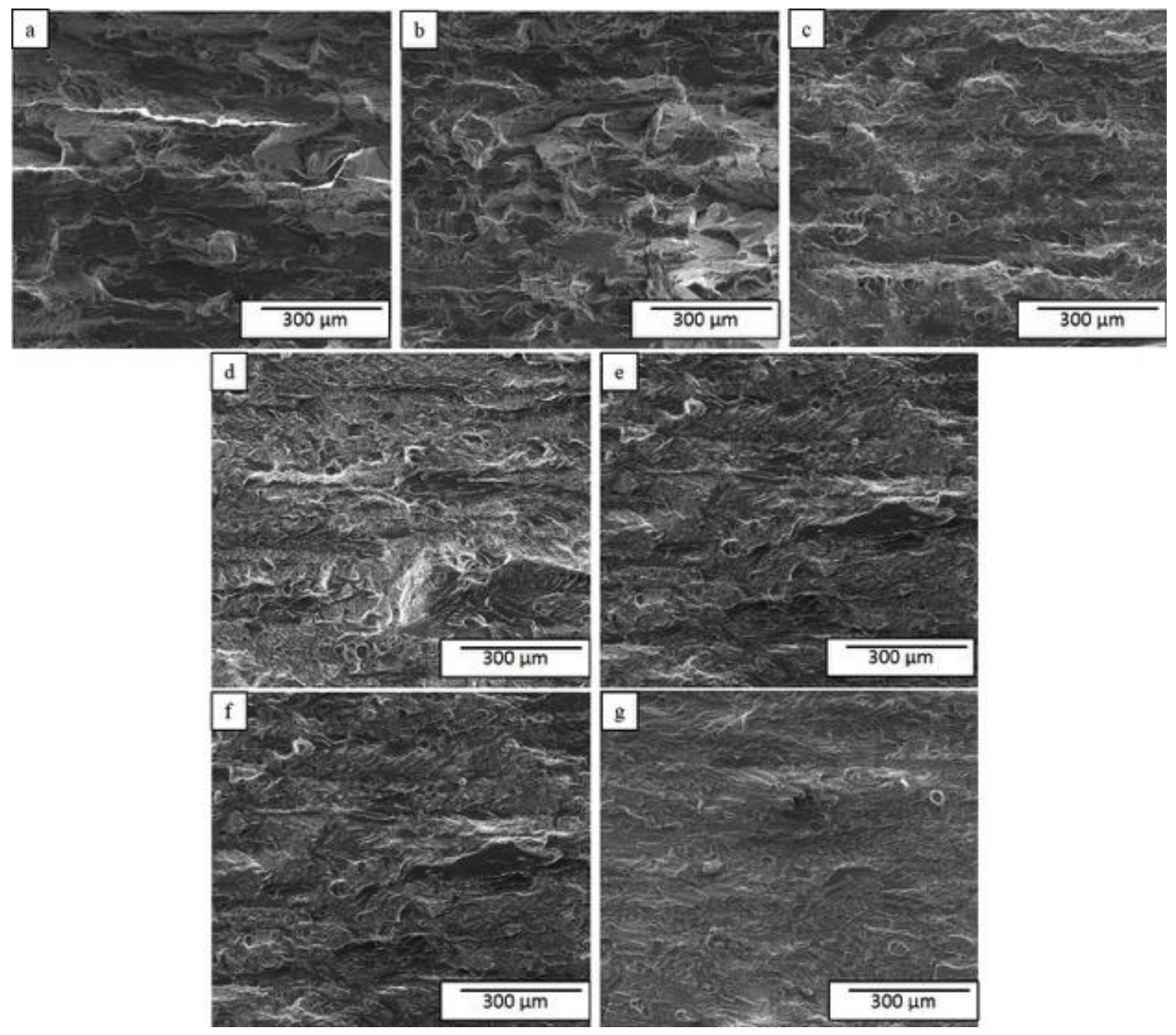

Figure 31: SEM images of the fatigue crack surface of 2199-T86 tested at constant $\mathrm{R}=0.5, f=$ $20 \mathrm{~Hz}$ and constant $\Delta \mathrm{K}=9 \mathrm{MPa} \sqrt{\mathrm{m}}$ (from Figure 22) for an L-T oriented specimen exposed to $\mathrm{P}_{\mathrm{H} 2 \mathrm{O}}$ of: (a) UHV (b) 0.5, (c) 1.8, (d) 4, (e) 18, (f) 38, and (g) $2668 \mathrm{~Pa}$. Crack growth is from left to right in each image. 


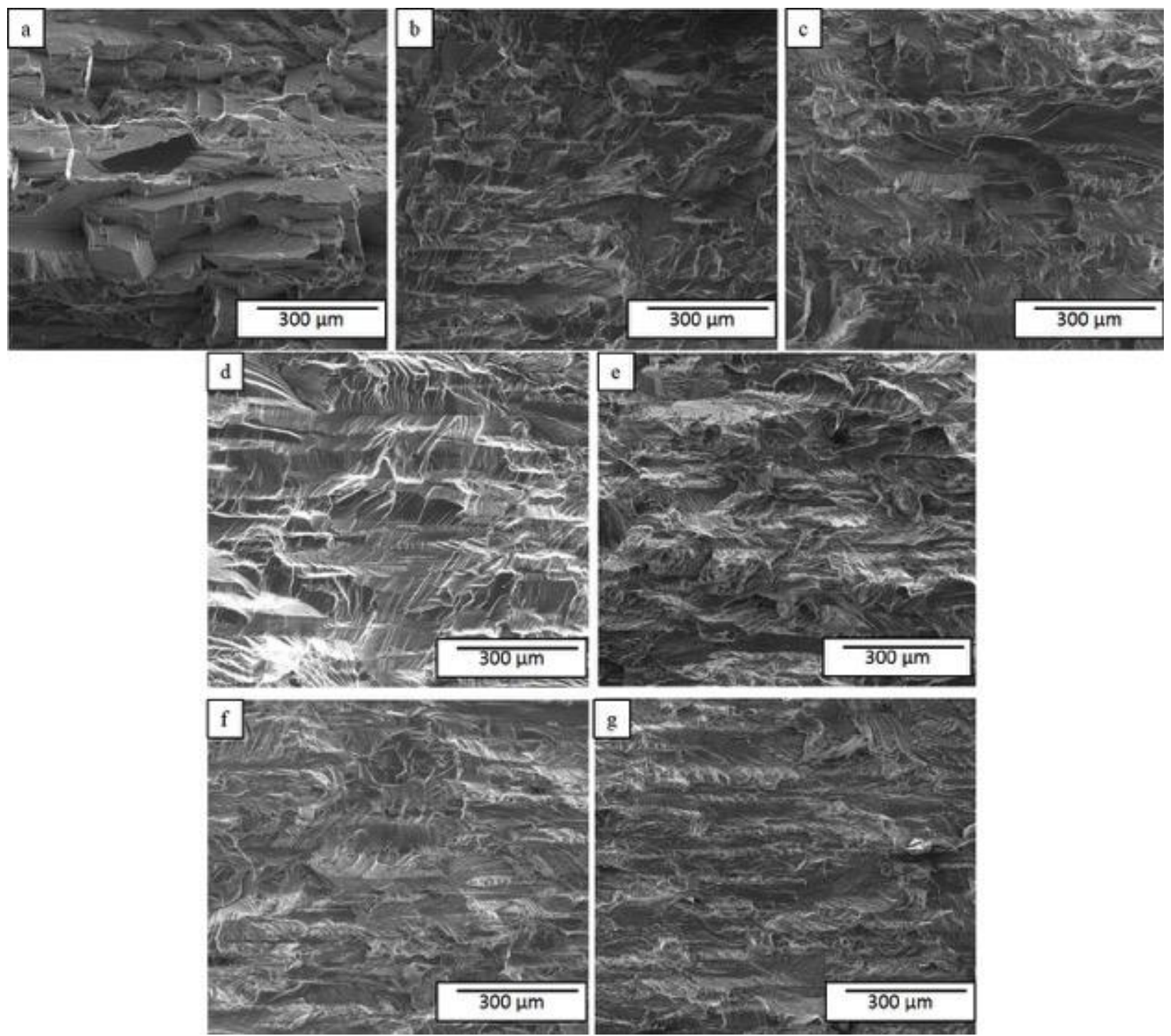

Figure 32: SEM images of the fatigue crack surface of 2199-T86 tested at constant $\mathrm{R}=0.5, f=$ $20 \mathrm{~Hz}$ and constant $\Delta \mathrm{K}=5 \mathrm{MPa} \sqrt{\mathrm{m}}$ (from Figure 22) for an L-T oriented specimen exposed to $\mathrm{P}_{\mathrm{H} 2 \mathrm{O}}$ of: (a) UHV (b) 0.5, (c) 1.8, (d) 4, (e) 18, (f) 38, and (g) $2668 \mathrm{~Pa}$. Crack growth is from left to right in each image. 

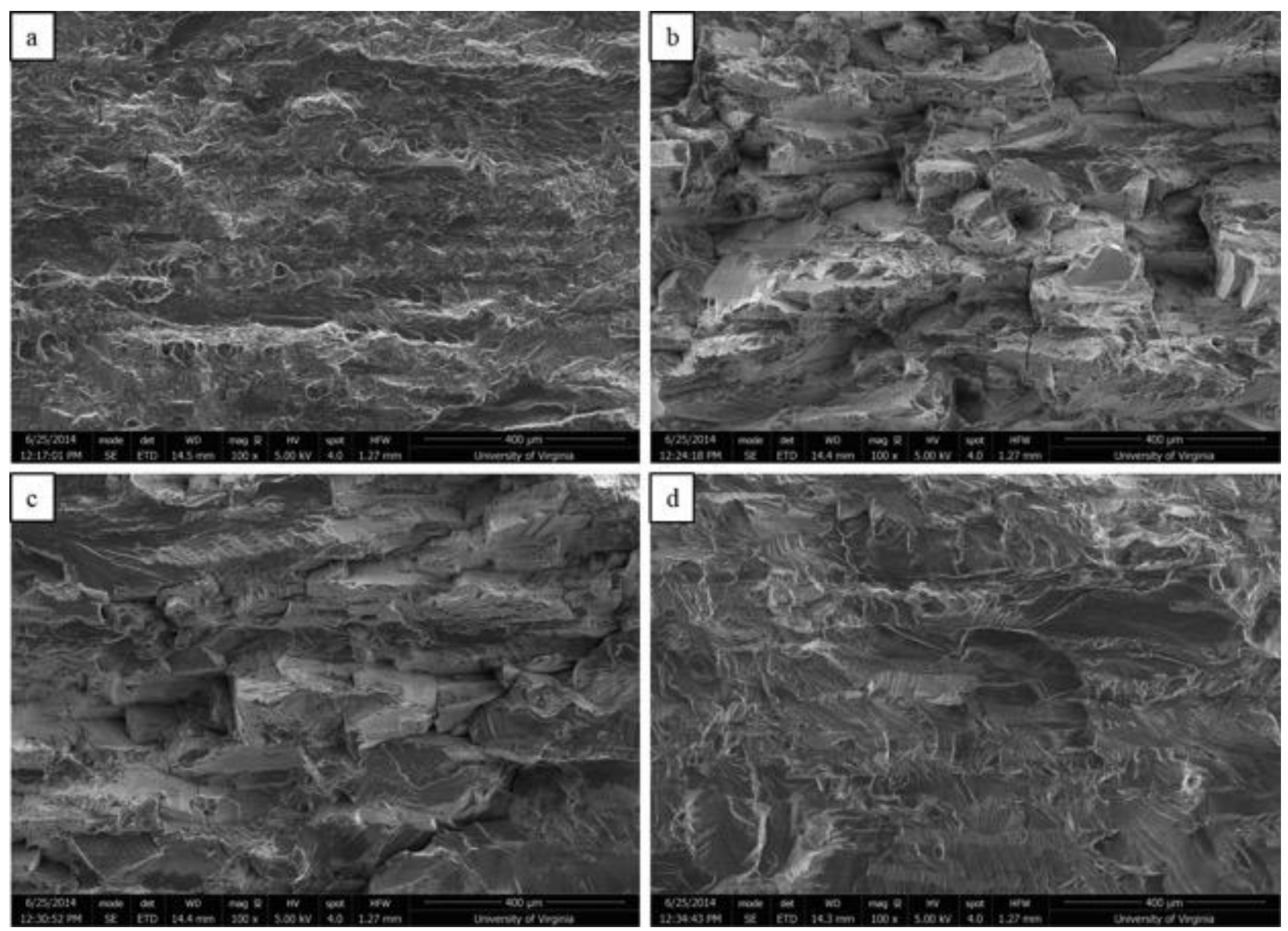

Figure 33: $\quad$ SEM images of the fatigue crack surface of 2199 - T86 tested under decreasing $\Delta K$ loading at constant $\mathrm{R}=0.5, f=20$ for an $\mathrm{L}-\mathrm{T}$ oriented specimen exposed to $\mathrm{P}_{\mathrm{H} 2 \mathrm{O}}=1.8 \mathrm{~Pa}$ with $\Delta \mathrm{K}$ of: (a) $\sim 9$, (b) $\sim 7$, (c) $\sim 6$, and (d) $\sim 5 \mathrm{MPa} \sqrt{\mathrm{m}}$. The direction of crack growth is left to right in each image. 
(a) $0.5 \mathrm{~mm}$

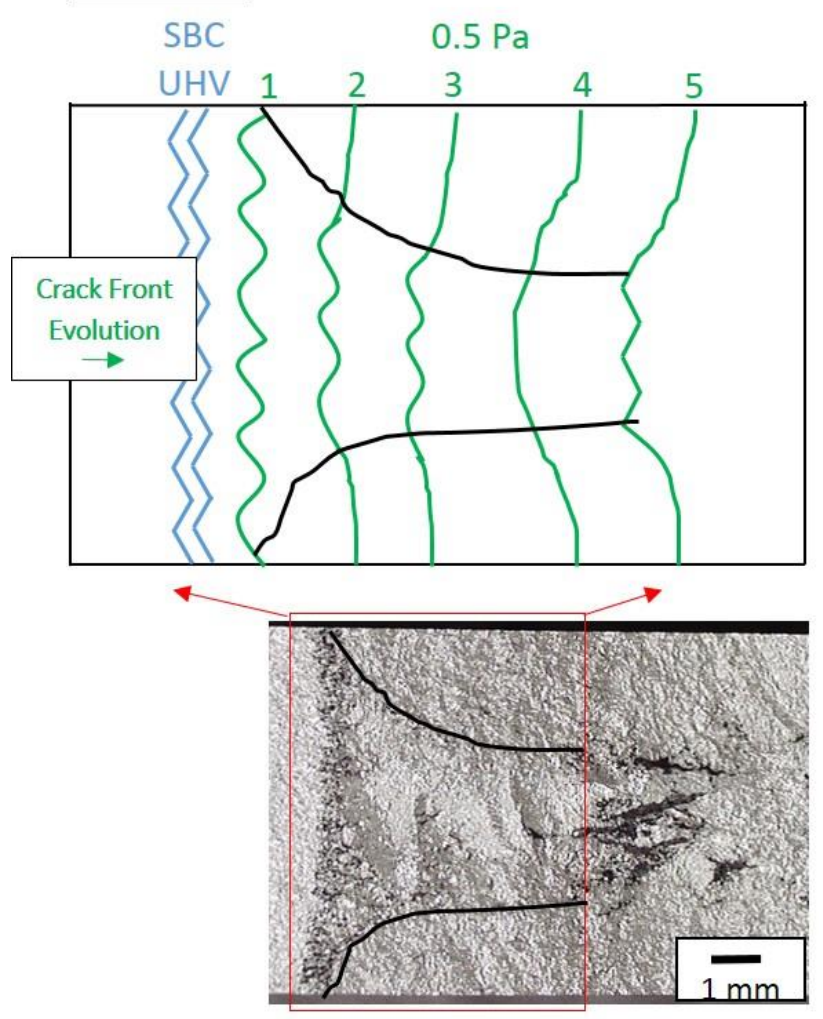

(b) $3 \mathrm{~mm}$
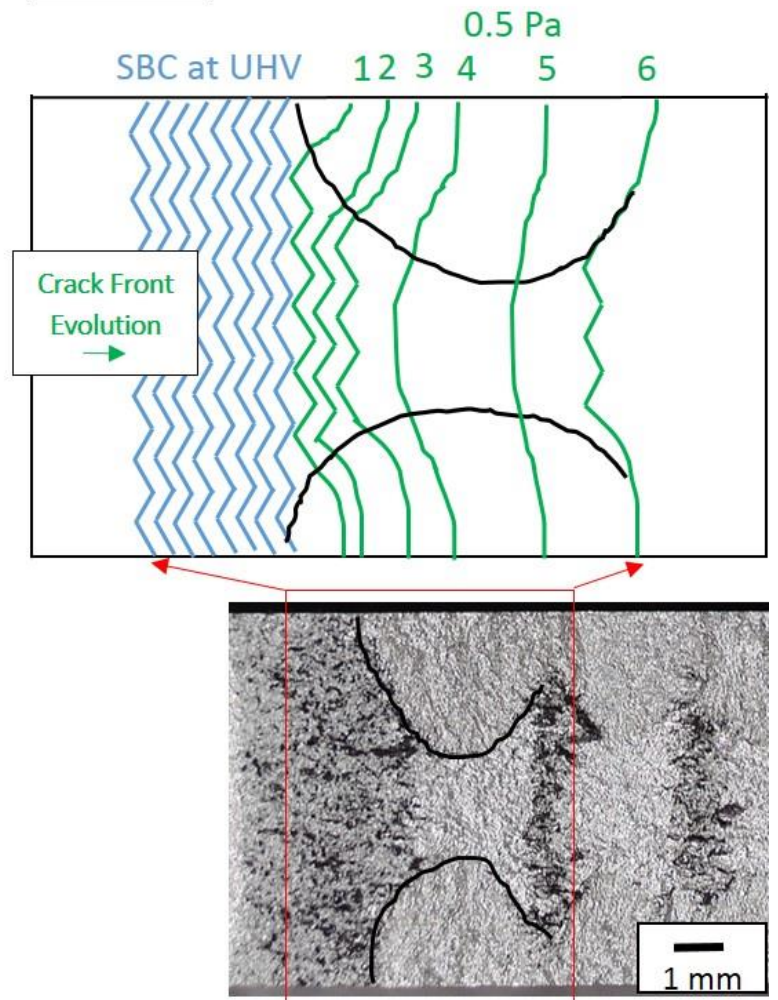

Roughness (SBC)

Transgranular

Numbers indicate continuous crack front progression Crack Growth $\longrightarrow$

Figure 34: (a) Schematic of crack front progression and corresponding optical fractograph stressed in pure water vapor at $\mathrm{P}_{\mathrm{H} 2 \mathrm{O}}=0.5 \mathrm{~Pa}$ at constant $\Delta \mathrm{K}=5 \mathrm{MPa} \sqrt{\mathrm{m}}$ and $\mathrm{R}$ of $0.50(\mathrm{f}=20$ $\mathrm{Hz}$ ) for 7075-T651 (L-T). $0.5 \mathrm{~mm}$ of crack wake roughness was imparted into the surface before beginning the exposure testing. (b) Schematic of crack front progression and corresponding optical fractograph stressed in pure water vapor at $\mathrm{P}_{\mathrm{H} 2 \mathrm{O}}=0.5 \mathrm{~Pa}$ at constant $\Delta \mathrm{K}=5 \mathrm{MPa} \sqrt{\mathrm{m}}$ and $\mathrm{R}$ of 0.50 ( $\mathrm{f}=20 \mathrm{~Hz}$ ) for 7075-T651 (L-T). $3 \mathrm{~mm}$ of crack wake roughness was imparted into the surface before beginning the exposure testing. Crack front evolution progresses left to right and each number corresponds to a continuous crack front. The increased roughness portion of the crack front is represented by triangles and the transgranular/flat portion of the crack front is identified by a straight line. The black line in both images outlines the increased roughness portion of the fracture surface. 


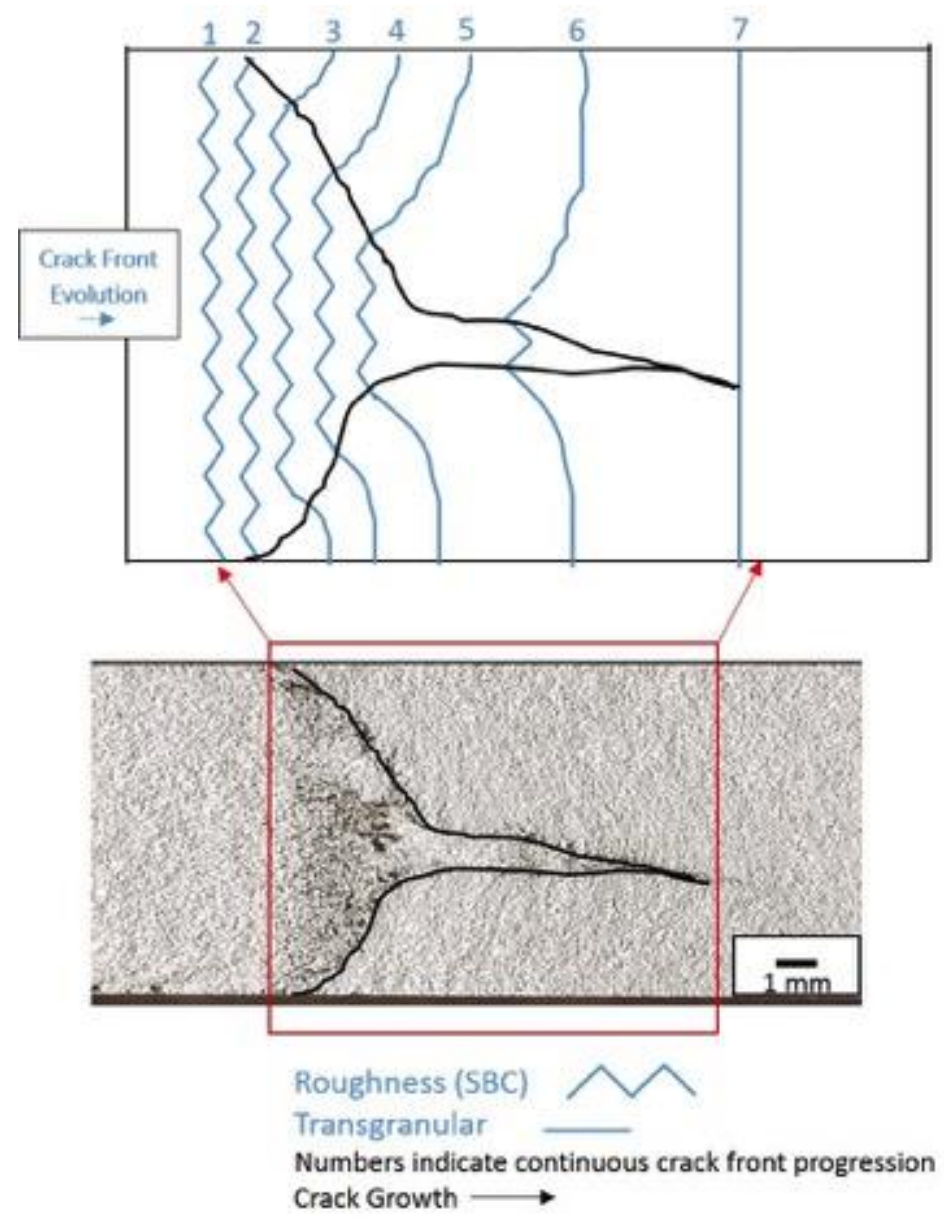

Figure 35: Schematic of crack front progression and corresponding optical fractograph stressed in pure water vapor at $\mathrm{P}_{\mathrm{H} 2 \mathrm{O}}=0.5 \mathrm{~Pa}$. For constant $\mathrm{R}, \Delta \mathrm{K}$ decreased with increasing crack length from right to left in this image. Crack front evolution progresses left to right and each number corresponds to a continuous crack front. The increased roughness portion of the crack front is represented by triangles and the transgranular/flat portion of the crack front is identified by a straight line. The black line in both images outlines the increased roughness portion of the fracture surface. 

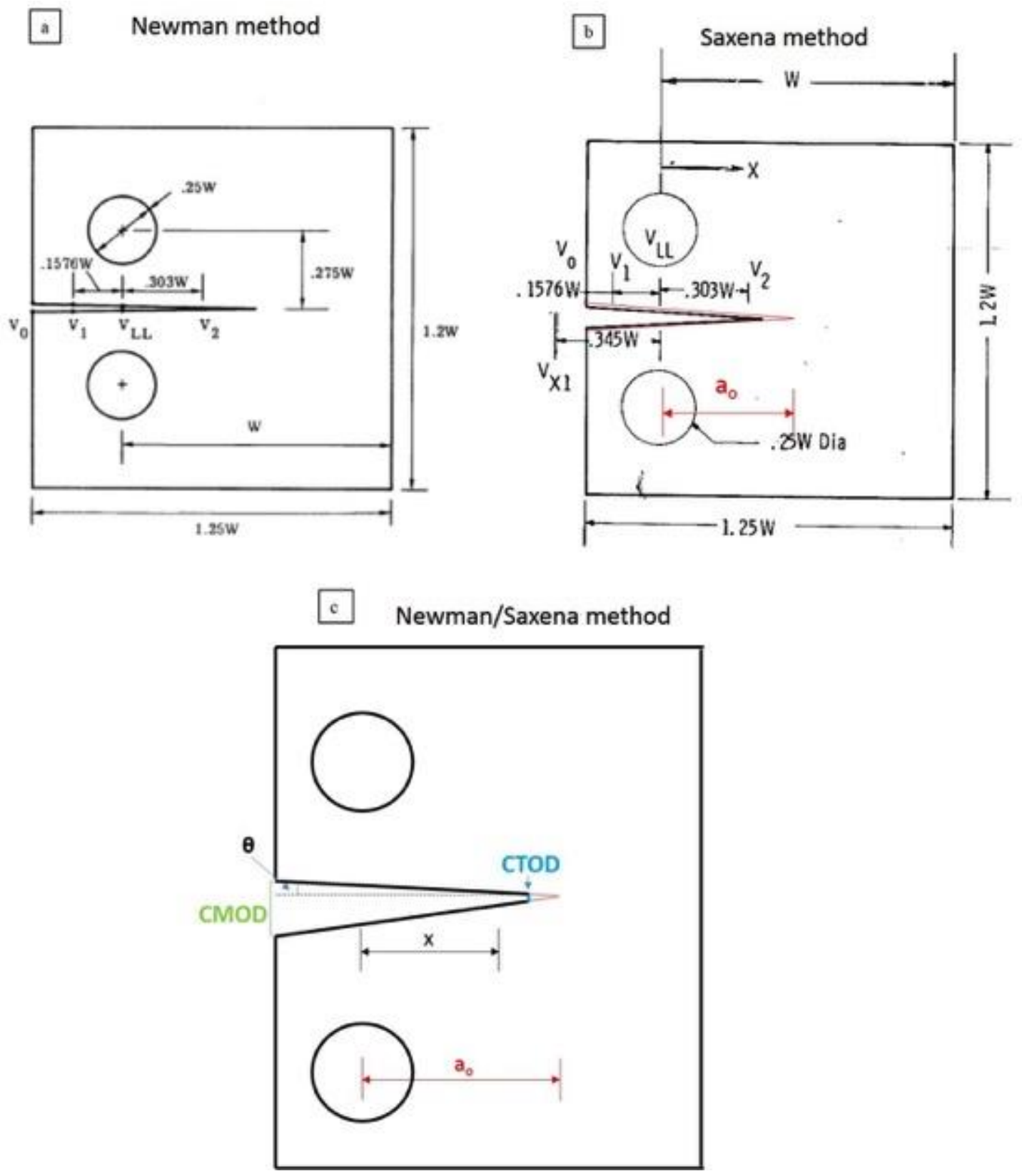

Figure 36: (a) $\mathrm{C}(\mathrm{T})$ specimen geometry and various locations where crack-line displacements were calculated according to the Newman method [75]. Newman represented the crack face profile from the crack tip to the load line as a straight line. (b) C(T) specimen geometry and various locations where crackline displacements were calculated according to the Saxena method [76]. The red lines represent the crack face profile for a crack length of $\mathrm{a}_{0}$ which is used to represent the elastic behavior of crack growth near the crack tip. $\mathrm{V}_{0}$ corresponds to the crack mouth opening for both diagrams. (c) Crack profile schematic of the combined model and $\mathrm{C}(\mathrm{T})$ specimen geometry that calculates crack mouth opening displacement (CMOD) using elastic theory and linear modeling of the crack surface: $\mathrm{x}$ is a specific crack length location of interest along the crack wake, $a_{0}$ is the current crack length factoring in the axis of rotation described previously, and $\theta$ is the angle of displacement between the crack surface and the crack tip opening displacement (CTOD). 

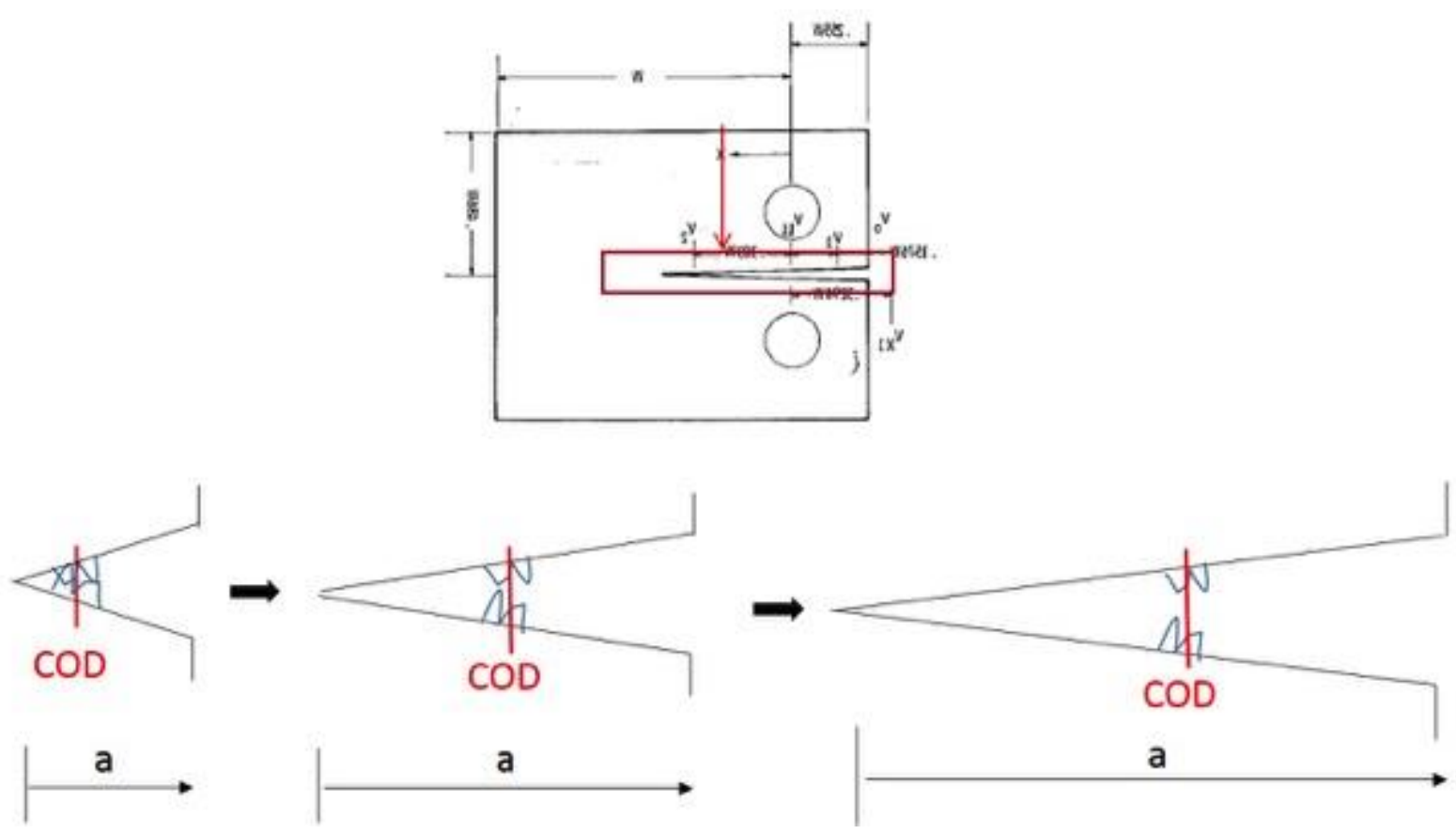

Figure 37: Schematic of a $\mathrm{C}(\mathrm{T})$ specimen [53] and a $\mathrm{C}(\mathrm{T})$ fatigue crack growing with a portion of increased roughness in the crack wake. As the crack continues to grow (left to right in the diagram above), the COD calculations still reflect the crack-opening displacement at the location of roughness indicated by the red line.

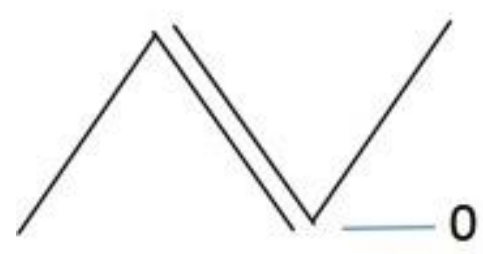

a

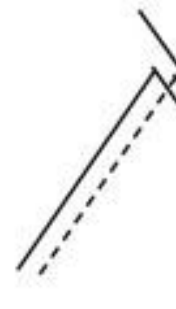

$\mathrm{b}$
$0.2 \mathrm{Rpv}$

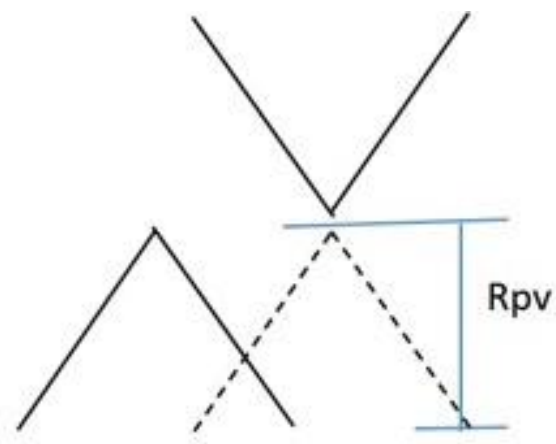

Figure 38: (a) Crack asperity schematic illustrating a condition where the Mode II displacement is equal to the Mode I displacement. This condition would represent the minimum or lower bound of Mode II displacement that would cause asperity contact. (b) Crack asperity schematic illustrating a condition where the amount of Mode II displacement results in $80 \%$ of the crack asperity height remaining in contact with the opposing face during loading. This situation was quantified as having a magnitude of $20 \%$ of the total crack asperity height $(0.2 * \mathrm{Rpv})$. (c) Crack asperity schematic illustrating a condition where the Mode II displacement results in opposing crack asperities that are directly aligned, resulting in peaks and valleys directly opening and closing on top of each other. This condition would represent the maximum or upper bound of Mode II displacement that would cause asperity contact. The magnitude of the total crack asperity height ( $\mathrm{Rpv}$ ) was used to quantify this displacement condition with the localized roughness. 

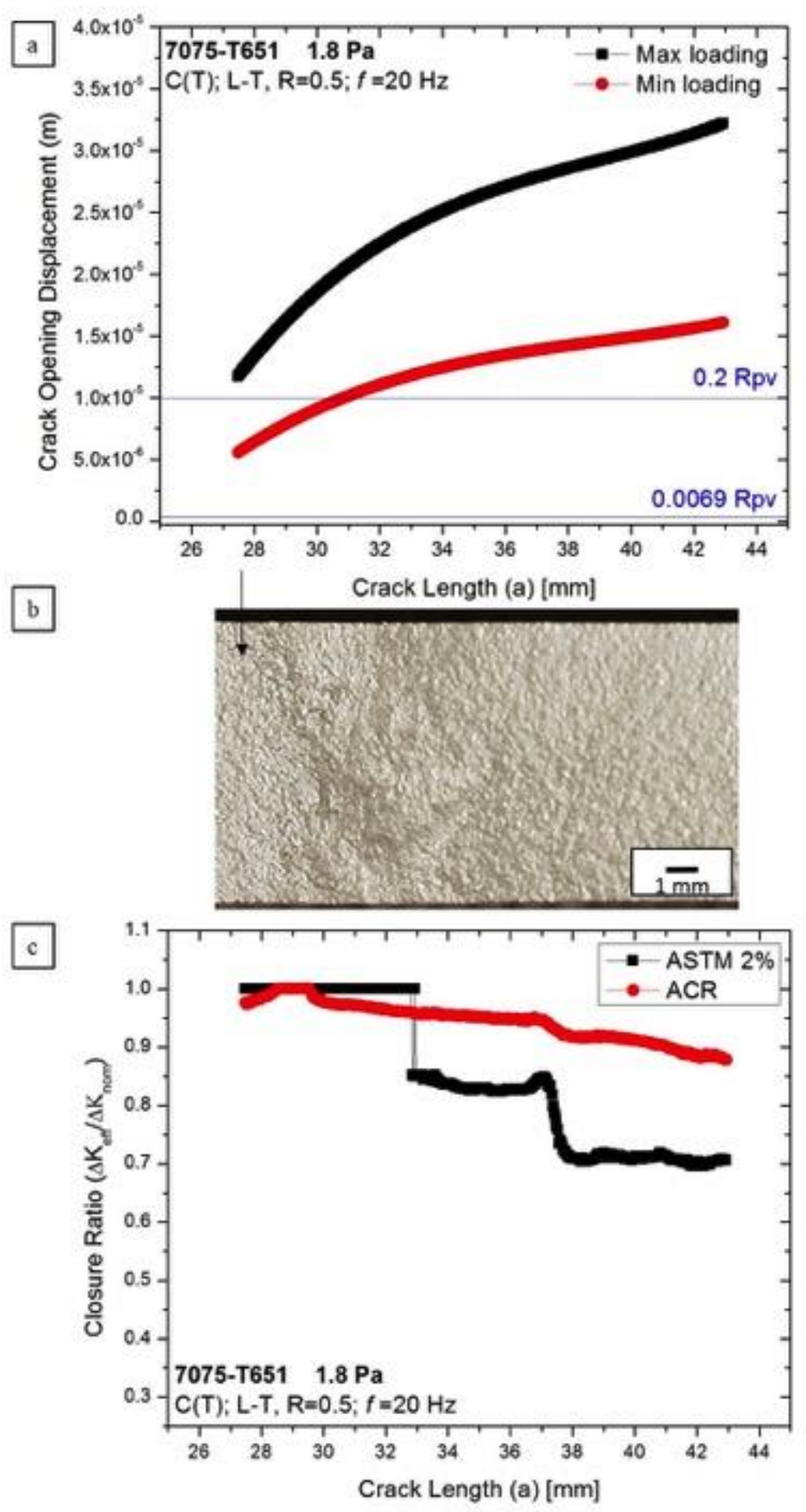

Figure 39: (a) Crack-opening displacement (COD) calculations at the location corresponding to the start of increased roughness $(\mathrm{a}=27.5 \mathrm{~mm})$ for the $1.8 \mathrm{~Pa}$ exposure specimen plotted as a function of crack length under maximum and minimum loading. Additionally, the respective height of the crack asperities under two Mode II displacement conditions (0.2 Rpv and $0.0069 \mathrm{Rpv})$ are plotted. (b) Optical fractograph of the test specimen corresponding to the crack length scale of the above and below plots with the black arrow indicating the location where the COD was calculated. Crack growth occurred left to right. (c) Closure analysis (ASTM 2\% and ACR ratio) versus crack length. 

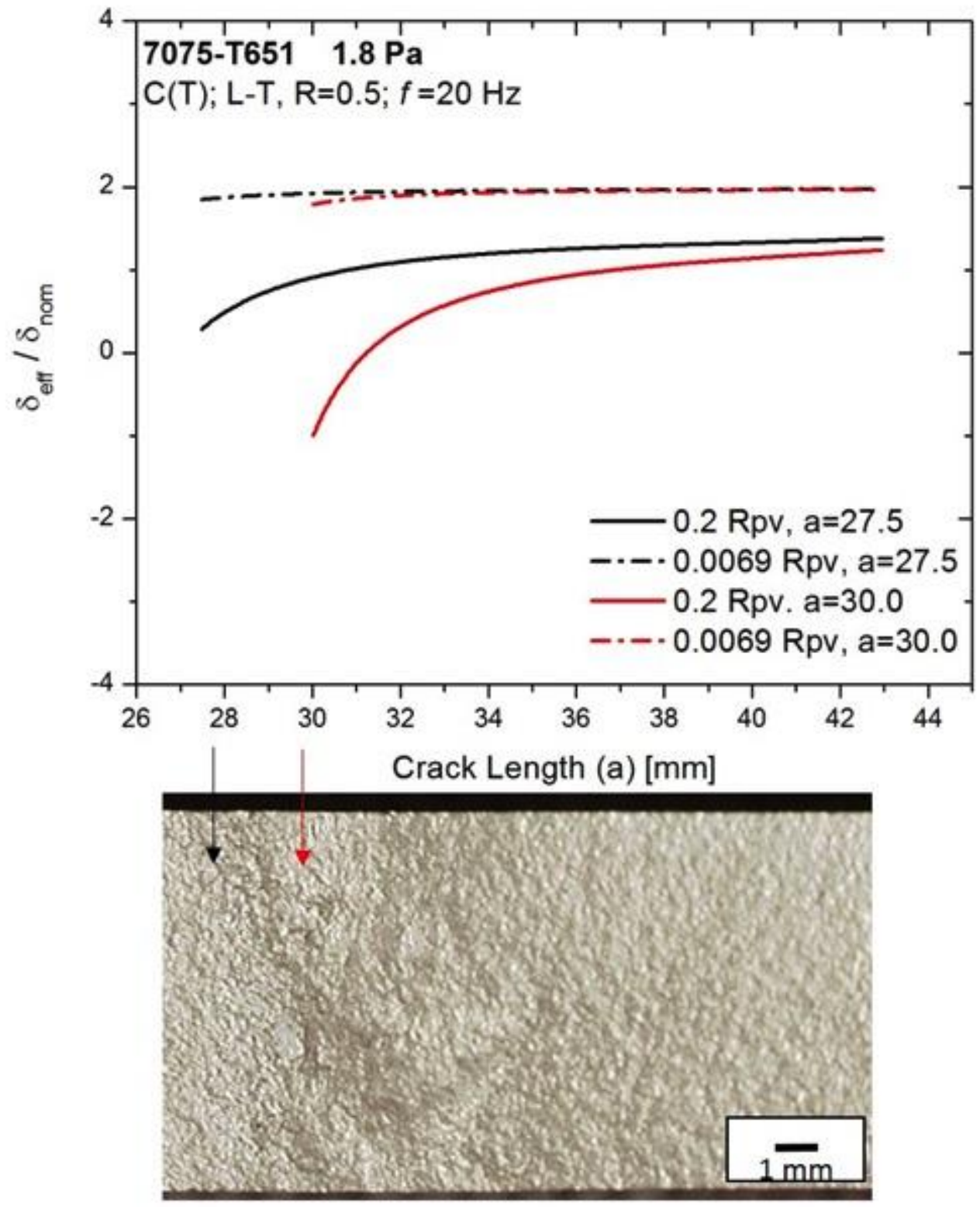

Figure 40: The effective crack opening $\left(\delta_{\text {eff }}\right)$ over the nominal crack opening $\left(\delta_{\text {nom }}=\right.$ COD at the maximum load - COD at the minimum load) is plotted as a function of crack length for two Mode II displacement conditions (0.2 Rpv and 0.0069 Rpv) and two locations ( $\mathrm{a}=27.5$ and 30.0 $\mathrm{mm}$ ) for the $1.8 \mathrm{~Pa}$ specimen. The optical fractograph of the test specimen, corresponding to the crack length scale of the above plot, indicates the two locations where the COD was calculated. 

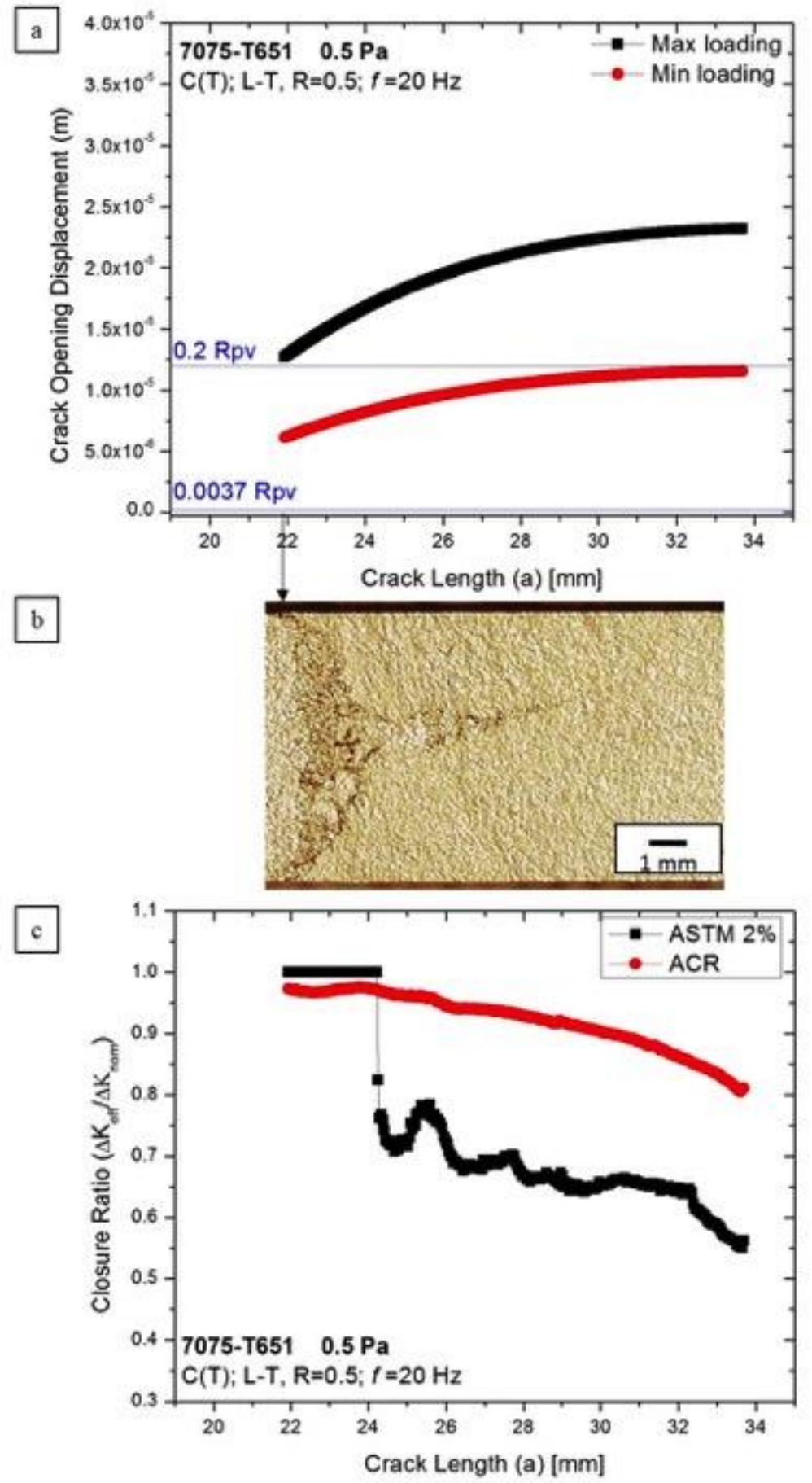

Figure 41: (a) Crack-opening displacement (COD) calculations at the location corresponding to the start of increased roughness $(\mathrm{a}=21.9 \mathrm{~mm})$ for the $0.5 \mathrm{~Pa}$ exposure specimen plotted as a function of crack length under maximum and minimum loading. Additionally, the respective height of the crack asperities under two Mode II displacement conditions ( $0.2 \mathrm{Rpv}$ and $0.0037 \mathrm{Rpv}$ ) are plotted. (b) Optical fractograph of the test specimen corresponding to the crack length scale of the above and below plots with the black arrow indicating the location where the COD was calculated. Crack growth occurred left to right. (c) Closure analysis (ASTM 2\% and ACR ratio) versus crack length. 


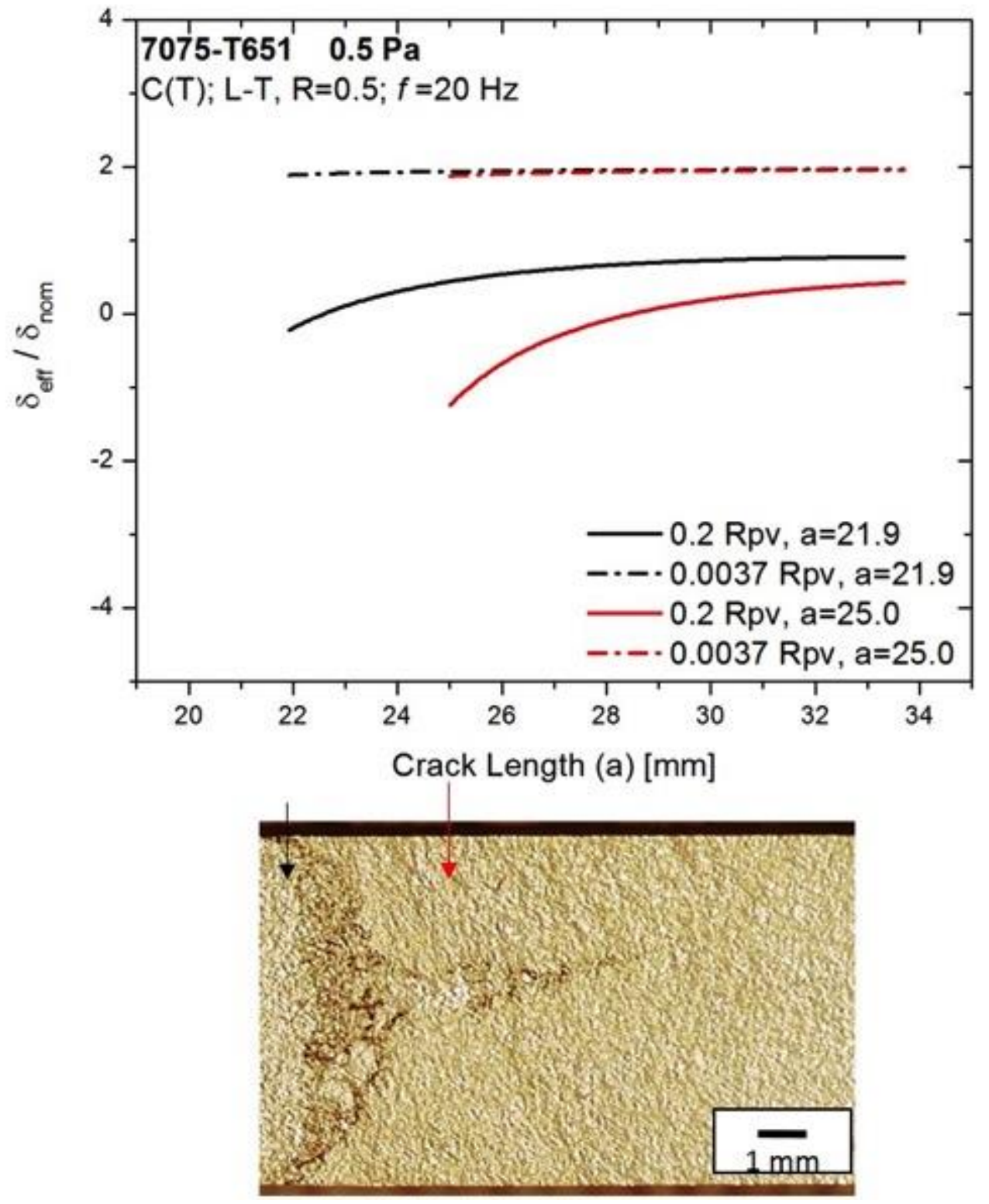

Figure 42: The effective crack opening $\left(\delta_{\text {eff }}\right)$ over the nominal crack opening $\left(\delta_{\text {nom }}=\right.$ COD at the maximum load - COD at the minimum load) is plotted as a function of crack length for two Mode II displacement conditions (0.2 Rpv and 0.0037 Rpv) and two locations ( $\mathrm{a}=27.5$ and $30.0 \mathrm{~mm}$ ) for the $0.5 \mathrm{~Pa}$ specimen. The optical fractograph of the test specimen, corresponding to the crack length scale of the above plot, indicates the two locations where the COD was calculated. 


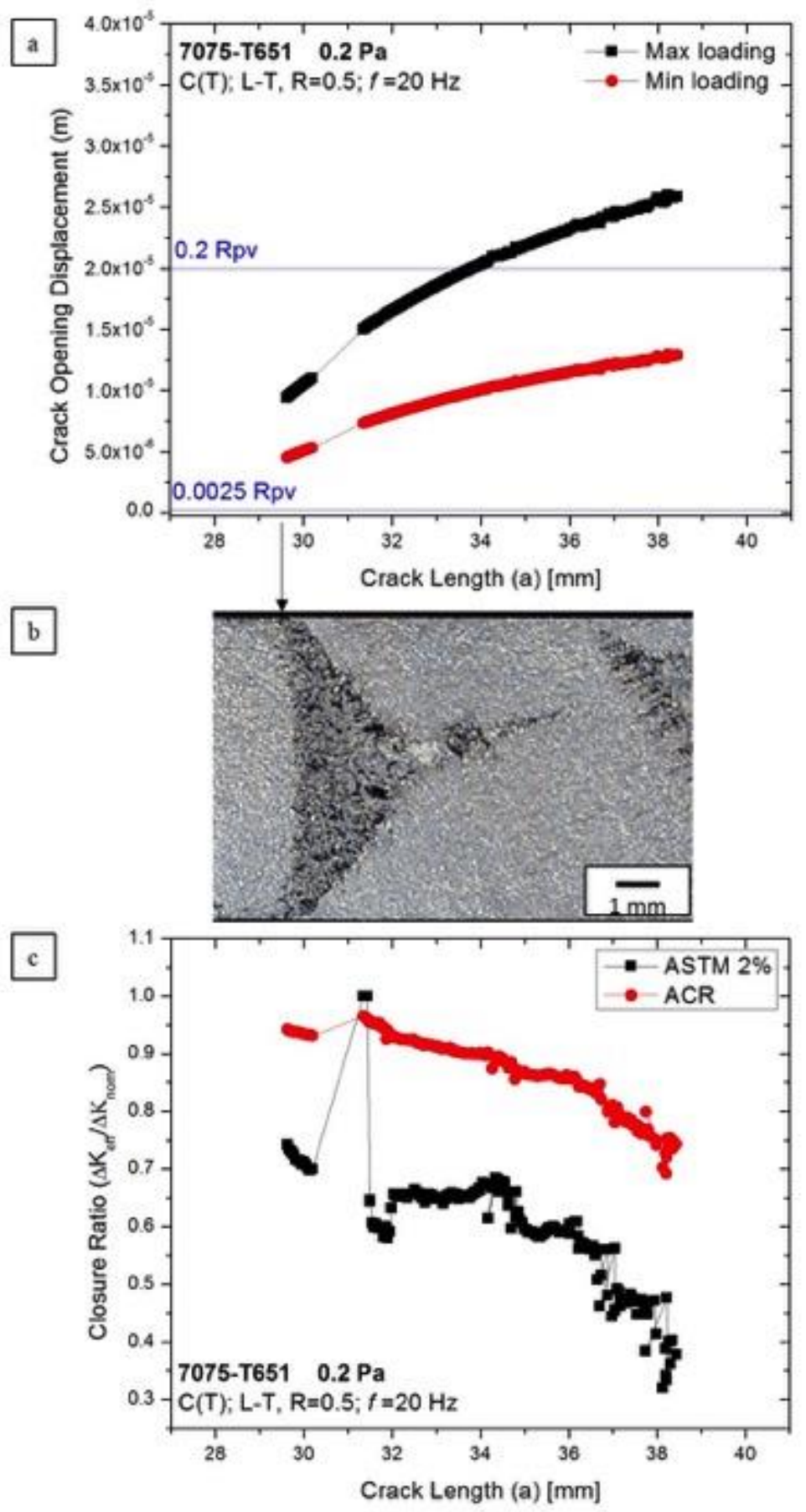

Figure 43: (a) Crack-opening displacement (COD) calculations at the location corresponding to the start of increased roughness $(\mathrm{a}=29.6 \mathrm{~mm})$ for the $0.2 \mathrm{~Pa}$ exposure specimen plotted as a function of crack length under maximum and minimum loading. Additionally, the respective height of the crack asperities under two Mode II displacement conditions (0.2 Rpv and $0.0069 \mathrm{Rpv}$ ) are plotted. (b) Optical fractograph of the test specimen corresponding to the crack length scale of the above and below plots with the black arrow indicating the location where the COD was calculated. Crack growth occurred left to right. (c) Closure analysis (ASTM 2\% and ACR ratio) versus crack length. 


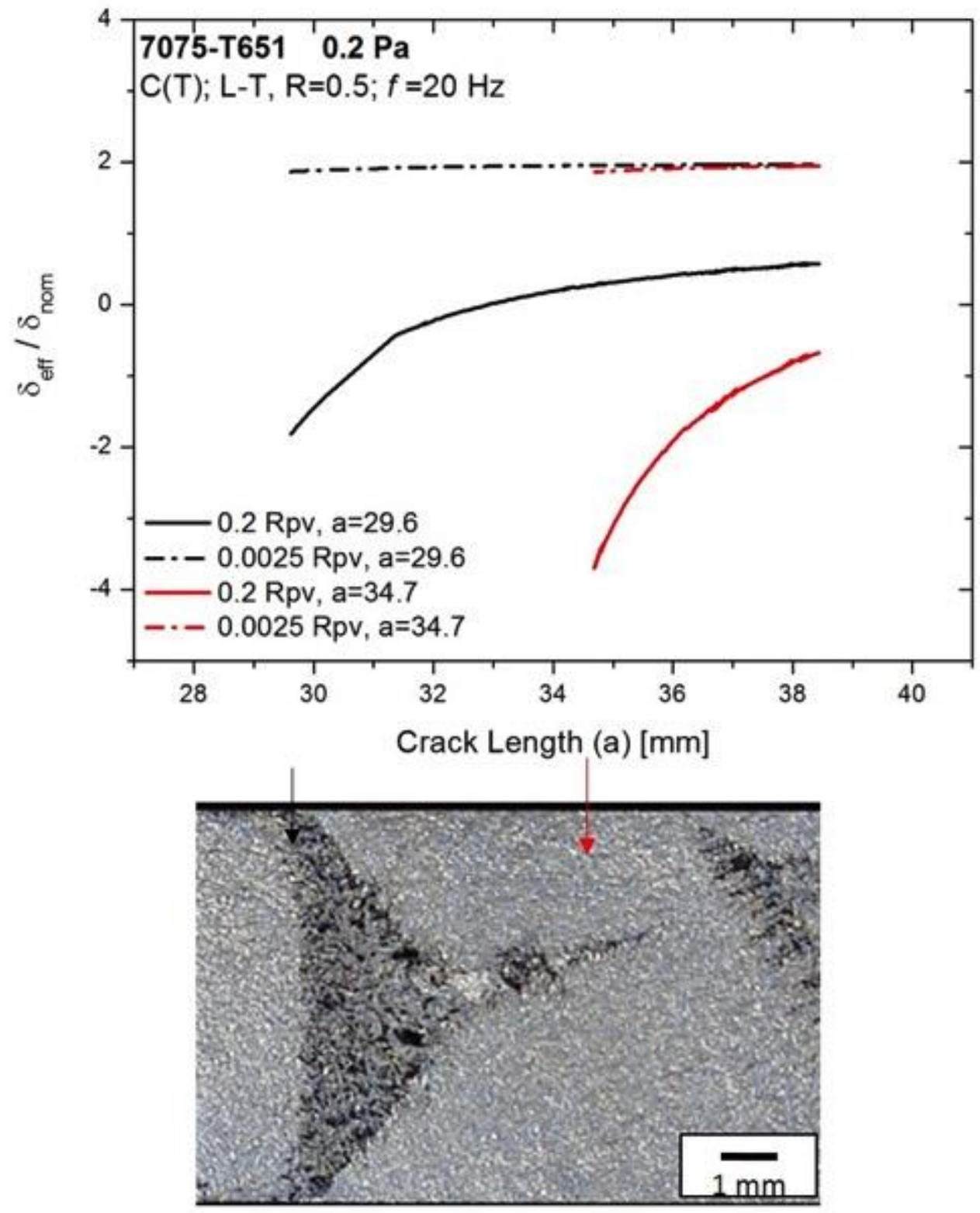

Figure 44: The effective crack opening $\left(\delta_{\text {eff }}\right)$ over the nominal crack opening $\left(\delta_{\text {nom }}=\right.$ COD at the maximum load - COD at the minimum load) is plotted as a function of crack length for two Mode II displacement conditions (0.2 Rpv and 0.0025 Rpv) and two locations (a=29.6 and 34.7 $\mathrm{mm}$ ) for the $0.2 \mathrm{~Pa}$ specimen. The optical fractograph of the test specimen, corresponding to the crack length scale of the above plot, indicates the two locations where the COD was calculated. 


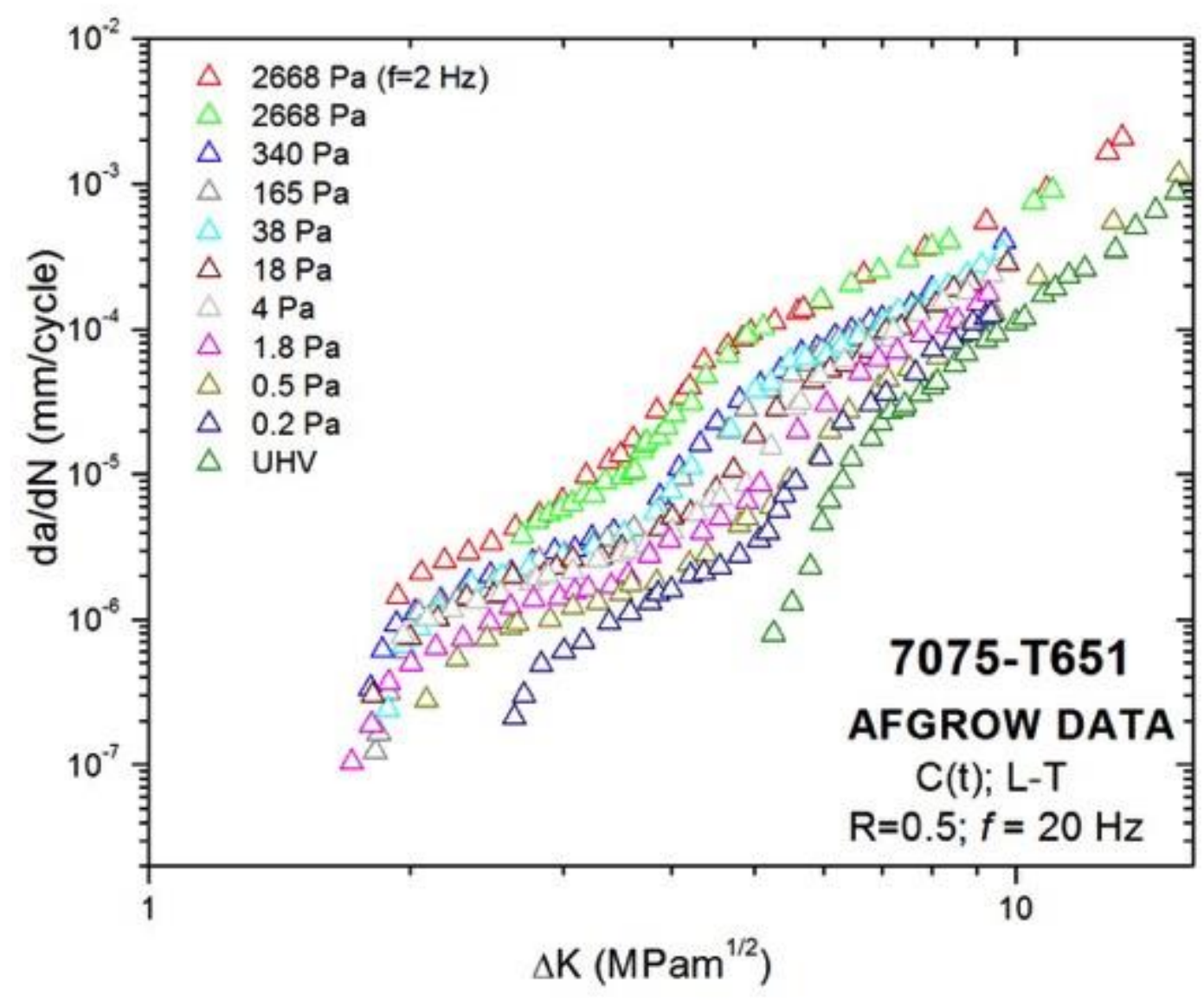

Figure 45: LEFM modeling (AFGROW) formatted fatigue crack growth rate versus decreasing $\Delta \mathrm{K}$ at constant $\mathrm{R}$ of $0.50(f=20 \mathrm{~Hz})$ for $7075-\mathrm{T} 651(\mathrm{~L}-\mathrm{T})$ at various-constant water vapor exposure levels from ultra-high vacuum $(0.25-0.50 \mu \mathrm{Pa}-\mathrm{s})$ to $1.3 \mathrm{kPa}-\mathrm{s}\left(\mathrm{P}_{\mathrm{H} 2 \mathrm{O}}=26.7 \mathrm{kPa}\right)$. The plotted data represents 30 data point sets (Table 8 ) that are taken from the results from Figure 1 . The threshold transition regime has been removed for the $1.8,0.5$, and $0.2 \mathrm{~Pa}$ exposures. 


\section{Tables}

Table 1: Weighted average $\mathrm{Ra}$ and $\mathrm{Rq}$ values taken in the threshold transition regime for $0.2,0.5$, and $1.8 \mathrm{~Pa}$

\begin{tabular}{|c|c|c|}
\hline $\mathrm{P}_{\mathrm{H} 20}(\mathrm{~Pa})$ & $\mathrm{Ra}(\mu \mathrm{m})$ & $\mathrm{Rq}(\mu \mathrm{m})$ \\
\hline 0.2 & 20.0 & 23.9 \\
\hline 0.5 & 10 & 12.2 \\
\hline 1.8 & 8.4 & 10.3 \\
\hline
\end{tabular}

Table 2: Weighted average $\mathrm{Ra}$ and $\mathrm{Rq}$ values taken after the threshold transition regime for 0.2 , 0.5 , and $1.8 \mathrm{~Pa}$

\begin{tabular}{|c|c|c|}
\hline $\mathrm{P}_{\mathrm{H} 20}(\mathrm{~Pa})$ & $\mathrm{Ra}(\mu \mathrm{m})$ & $\mathrm{Rq}(\mu \mathrm{m})$ \\
\hline 0.2 & 5.4 & 7.0 \\
\hline 0.5 & 7.2 & 8.7 \\
\hline 1.8 & 9.2 & 11.2 \\
\hline
\end{tabular}

Table 3: Weighted average $\mathrm{Ra}$ and $\mathrm{Rq}$ values taken for 4 and $2668 \mathrm{~Pa}$

\begin{tabular}{|c|c|c|}
\hline $\mathrm{P}_{\mathrm{H} 20}(\mathrm{~Pa})$ & $\mathrm{Ra}(\mu \mathrm{m})$ & $\mathrm{Rq}(\mu \mathrm{m})$ \\
\hline 4 & 7.7 & 10 \\
\hline 2668 & 6.7 & 8.2 \\
\hline
\end{tabular}

Table 4: Largest $\mathrm{Rp}$ and $\mathrm{Rv}$ values observed in the threshold transition regime for $0.2,0.5$, and 1.8 $\mathrm{Pa} . \mathrm{Rpv}$ is the sum of $\mathrm{Rp}$ and $\mathrm{Rv}$ and reflects the total magnitude of the crack face roughness.

\begin{tabular}{|c|c|c|c|}
\hline $\mathrm{P}_{\mathrm{H} 20}(\mathrm{~Pa})$ & $\mathrm{Rp}(\mu \mathrm{m})$ & $\mathrm{Rv}(\mu \mathrm{m})$ & $\mathrm{Rpv}(\mu \mathrm{m})$ \\
\hline 0.2 & 45.3 & 46.5 & 91.8 \\
\hline 0.5 & 46.0 & 24.6 & 70.6 \\
\hline 1.8 & 23.2 & 26.6 & 49.8 \\
\hline
\end{tabular}


Table 5: The magnitude of the fracture surface roughness span for the $0.2,0.5$, and $1.8 \mathrm{~Pa}$ exposures

\begin{tabular}{|l|l|}
\hline $\mathrm{P}_{\mathrm{H} 20}(\mathrm{~Pa})$ & Span of SBC $(\mathrm{mm})$ \\
\hline 0.2 & 5.1 \\
\hline 0.5 & 3.1 \\
\hline 1.8 & 2.5 \\
\hline
\end{tabular}

Table 6: The CTOD, Mode II displacement, and the corresponding percentage of Rpv for the condition where Mode II/Mode $\mathrm{I}=0.3$ for the $0.2,0.5$, and $1.8 \mathrm{~Pa}$ exposures. These values were calculated at crack lengths corresponding to the start of the roughness span.

\begin{tabular}{|l|l|l|l|l|}
\hline$P_{\mathrm{H} 20}(\mathrm{~Pa})$ & $\begin{array}{l}\text { CTOD max } \\
(\mathrm{m})\end{array}$ & $\begin{array}{l}\text { Mode II } \\
\text { Displacement }(\mathrm{m})\end{array}$ & Rpv (m) & $\begin{array}{l}\text { Mode II } \\
\text { Displacement/Rpv }\end{array}$ \\
\hline 0.2 & $7.2 \times 10^{-7}$ & $2.4 \times 10^{-7}$ & $9.2 \times 10^{-5}$ & 0.00259 \\
\hline 0.5 & $8.8 \times 10^{-7}$ & $2.7 \times 10^{-7}$ & $7.1 \times 10^{-5}$ & 0.00375 \\
\hline 1.8 & $1.1 \times 10^{-6}$ & $3.4 \times 10^{-7}$ & $5.0 \times 10^{-5}$ & 0.00689 \\
\hline
\end{tabular}

Table 7: The 3 Mode II displacement conditions and the corresponding magnitude of the crack asperities for the $0.2,0.5$, and $1.8 \mathrm{~Pa}$ exposures.

\begin{tabular}{|c|c|c|c|c|}
\hline $\begin{array}{c}\mathrm{P}_{\mathrm{H} 20} \\
(\mathrm{~Pa})\end{array}$ & $\begin{array}{c}\text { Rpv (m) } \\
\text { (Figure 38c) }\end{array}$ & $\begin{array}{c}0.2 \mathrm{Rpv}(\mathrm{m}) \\
(\text { Figure 38b) }\end{array}$ & $\begin{array}{c}\text { Mode II/Mode I =0.3 } \\
\text { (in terms of Rpv) } \\
(\text { Table 6) }\end{array}$ & $\begin{array}{c}\text { Mode II/Mode I =0.3 } \\
(\mathrm{m})\end{array}$ \\
\hline 0.2 & $9.2 \times 10^{-5}$ & $1.8 \times 10^{-5}$ & $0.00259^{*} \mathrm{Rpv}$ & $2.4 \times 10^{-7}$ \\
\hline 0.5 & $7.1 \times 10^{-5}$ & $1.4 \times 10^{-5}$ & $0.00375^{*} \mathrm{Rpv}$ & $2.6 \times 10^{-7}$ \\
\hline 1.8 & $5.0 \times 10^{-5}$ & $1.0 \times 10^{-5}$ & $0.00689^{*} \mathrm{Rpv}$ & $3.4 \times 10^{-7}$ \\
\hline
\end{tabular}




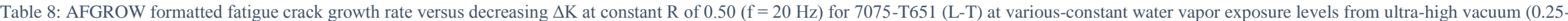

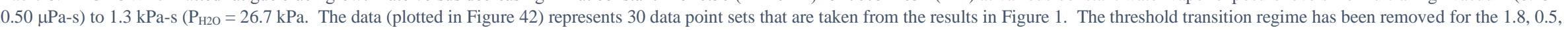
and $0.2 \mathrm{~Pa}$ exposures.

\begin{tabular}{|c|c|c|c|c|c|c|c|c|c|c|c|}
\hline $2668 \mathrm{~Pa}(2 \mathrm{~Hz})$ & & $2668 \mathrm{~Pa}$ & & $340 \mathrm{~Pa}$ & & $165 \mathrm{~Pa}$ & & $38 \mathrm{~Pa}$ & & $18 \mathrm{~Pa}$ & \\
\hline $\mathrm{da} / \mathrm{dN}$ (mm/cycle) & $\Delta \mathrm{K}(\mathrm{MPaVm})$ & $\mathrm{da} / \mathrm{dN}$ & $\Delta K$ & $\mathrm{da} / \mathrm{dN}$ & $\Delta K$ & $\mathrm{da} / \mathrm{dN}$ & $\Delta \mathrm{K}$ & $\mathrm{da} / \mathrm{dN}$ & $\Delta \mathrm{K}$ & $\mathrm{da} / \mathrm{dN}$ & $\Delta \mathrm{K}$ \\
\hline $1.44 \mathrm{E}-06$ & 1.94 & $3.74 \mathrm{E}-06$ & 2.69 & $3.33 \mathrm{E}-07$ & 1.80 & $1.22 \mathrm{E}-07$ & 1.82 & $2.39 \mathrm{E}-07$ & 1.89 & $3.02 \mathrm{E}-07$ & 1.81 \\
\hline $2.08 \mathrm{E}-06$ & 2.06 & 4.75E-06 & 2.80 & $6.15 \mathrm{E}-07$ & 1.86 & 1.64E-07 & 1.84 & $6.65 \mathrm{E}-07$ & 1.97 & $7.44 \mathrm{E}-07$ & 2.00 \\
\hline $2.52 \mathrm{E}-06$ & 2.19 & $5.32 \mathrm{E}-06$ & 2.89 & $9.19 \mathrm{E}-07$ & 1.93 & $3.13 \mathrm{E}-07$ & 1.89 & 8.69E-07 & 2.05 & $1.01 \mathrm{E}-06$ & 2.15 \\
\hline $2.91 \mathrm{E}-06$ & 2.33 & $5.70 \mathrm{E}-06$ & 2.98 & $1.1 \mathrm{E}-06$ & 2.03 & $6.04 \mathrm{E}-07$ & 1.98 & $1.14 \mathrm{E}-06$ & 2.15 & $1.40 \mathrm{E}-06$ & 2.32 \\
\hline $3.36 \mathrm{E}-06$ & 2.48 & $6.29 \mathrm{E}-06$ & 3.08 & $1.35 \mathrm{E}-06$ & 2.17 & $1.11 \mathrm{E}-06$ & 2.05 & $1.75 \mathrm{E}-06$ & 2.35 & $1.45 \mathrm{E}-06$ & 2.50 \\
\hline $4.28 \mathrm{E}-06$ & 2.65 & 7.19E-06 & 3.17 & $1.81 \mathrm{E}-06$ & 2.34 & $1.28 \mathrm{E}-06$ & 2.16 & $1.99 \mathrm{E}-06$ & 2.55 & 1.97E-06 & 2.63 \\
\hline 5.19E-06 & 2.82 & $7.22 \mathrm{E}-06$ & 3.26 & 2.01E-06 & 2.47 & $1.37 \mathrm{E}-06$ & 2.27 & $2.27 \mathrm{E}-06$ & 2.74 & $2.01 \mathrm{E}-06$ & 2.86 \\
\hline $6.53 \mathrm{E}-06$ & 3.00 & $8.85 \mathrm{E}-06$ & 3.36 & 2.07E-06 & 2.62 & $1.58 \mathrm{E}-06$ & 2.36 & $2.72 \mathrm{E}-06$ & 3.00 & $2.28 \mathrm{E}-06$ & 2.95 \\
\hline $9.71 \mathrm{E}-06$ & 3.19 & $9.52 \mathrm{E}-06$ & 3.51 & $2.5 \mathrm{E}-06$ & 2.77 & $1.82 \mathrm{E}-06$ & 2.50 & $3.15 \mathrm{E}-06$ & 3.26 & $2.58 \mathrm{E}-06$ & 3.08 \\
\hline $1.22 \mathrm{E}-05$ & 3.40 & $1.02 \mathrm{E}-05$ & 3.60 & $2.94 \mathrm{E}-06$ & 2.94 & 2.03E-06 & 2.61 & $3.84 \mathrm{E}-06$ & 3.53 & 2.69E-06 & 3.32 \\
\hline $1.36 \mathrm{E}-05$ & 3.50 & $1.05 \mathrm{E}-05$ & 3.64 & $3.01 \mathrm{E}-06$ & 3.10 & $2.54 \mathrm{E}-06$ & 2.82 & $5.45 \mathrm{E}-06$ & 3.84 & $3.12 \mathrm{E}-06$ & 3.51 \\
\hline $1.72 \mathrm{E}-05$ & 3.62 & $1.44 \mathrm{E}-05$ & 3.70 & 3.63E-06 & 3.25 & $2.81 \mathrm{E}-06$ & 3.01 & 7.69E-06 & 4.00 & $4.22 \mathrm{E}-06$ & 3.89 \\
\hline $2.75 \mathrm{E}-05$ & 3.85 & $1.59 \mathrm{E}-05$ & 3.72 & $3.98 \mathrm{E}-06$ & 3.43 & $3.31 \mathrm{E}-06$ & 3.26 & $1.12 \mathrm{E}-05$ & 4.23 & $5.09 \mathrm{E}-06$ & 4.01 \\
\hline 3.37E-05 & 4.11 & $1.65 \mathrm{E}-05$ & 3.75 & 7.02E-06 & 3.88 & $3.56 \mathrm{E}-06$ & 3.38 & $2.01 \mathrm{E}-05$ & 4.69 & $5.42 \mathrm{E}-06$ & 4.21 \\
\hline $4.01 \mathrm{E}-05$ & 4.20 & $1.79 \mathrm{E}-05$ & 3.87 & $1.1 \mathrm{E}-05$ & 4.09 & $4.22 \mathrm{E}-06$ & 3.62 & $3.73 \mathrm{E}-05$ & 5.00 & 7.91E-06 & 4.51 \\
\hline $5.98 \mathrm{E}-05$ & 4.37 & $2.10 \mathrm{E}-05$ & 3.95 & $1.6 \mathrm{E}-05$ & 4.33 & $5.74 \mathrm{E}-06$ & 3.88 & $4.25 \mathrm{E}-05$ & 5.22 & 1.07E-05 & 4.73 \\
\hline $7.55 \mathrm{E}-05$ & 4.65 & $2.58 \mathrm{E}-05$ & 4.04 & 2.27E-05 & 4.52 & $9.50 \mathrm{E}-06$ & 4.11 & $6.00 \mathrm{E}-05$ & 5.49 & $1.84 \mathrm{E}-05$ & 4.99 \\
\hline $8.50 \mathrm{E}-05$ & 4.84 & 3.09E-05 & 4.22 & $3.23 \mathrm{E}-05$ & 4.80 & 1.99E-05 & 4.66 & $6.25 \mathrm{E}-05$ & 5.72 & 2.82E-05 & 5.30 \\
\hline $9.48 \mathrm{E}-05$ & 4.95 & 4.74E-05 & 4.39 & 4.09E-05 & 5.07 & $2.80 \mathrm{E}-05$ & 4.89 & $6.63 \mathrm{E}-05$ & 6.04 & 4.39E-05 & 5.83 \\
\hline $1.11 \mathrm{E}-04$ & 5.27 & $6.54 \mathrm{E}-05$ & 4.66 & $5.14 \mathrm{E}-05$ & 5.35 & $3.78 \mathrm{E}-05$ & 5.21 & 7.70E-05 & 6.30 & $5.21 \mathrm{E}-05$ & 6.10 \\
\hline $1.31 \mathrm{E}-04$ & 5.61 & $8.91 \mathrm{E}-05$ & 4.89 & $6.81 \mathrm{E}-05$ & 5.66 & 4.83E-05 & 5.52 & $8.78 \mathrm{E}-05$ & 6.57 & 5.65E-05 & 6.39 \\
\hline $1.36 \mathrm{E}-04$ & 5.69 & $1.03 \mathrm{E}-04$ & 5.11 & $7.29 \mathrm{E}-05$ & 5.90 & 5.73E-05 & 5.75 & $9.27 \mathrm{E}-05$ & 6.89 & $6.36 \mathrm{E}-05$ & 6.62 \\
\hline $1.54 \mathrm{E}-04$ & 5.97 & 1.57E-04 & 5.95 & $8.75 \mathrm{E}-05$ & 6.18 & $6.28 \mathrm{E}-05$ & 5.86 & $1.08 \mathrm{E}-04$ & 7.05 & $7.08 \mathrm{E}-05$ & 6.81 \\
\hline $2.34 \mathrm{E}-04$ & 6.68 & $2.04 \mathrm{E}-04$ & 6.45 & $9.8 \mathrm{E}-05$ & 6.46 & $6.99 \mathrm{E}-05$ & 5.97 & $1.33 \mathrm{E}-04$ & 7.32 & $9.62 \mathrm{E}-05$ & 7.07 \\
\hline $3.62 \mathrm{E}-04$ & 7.85 & $2.51 \mathrm{E}-04$ & 6.95 & 0.000112 & 6.79 & 7.13E-05 & 6.15 & $1.41 \mathrm{E}-04$ & 7.71 & 1.03E-04 & 7.37 \\
\hline $5.41 \mathrm{E}-04$ & 9.23 & $3.00 \mathrm{E}-04$ & 7.50 & 0.000118 & 7.00 & $8.40 \mathrm{E}-05$ & 6.25 & $1.80 \mathrm{E}-04$ & 8.03 & $1.30 \mathrm{E}-04$ & 7.71 \\
\hline $9.12 \mathrm{E}-04$ & 10.85 & $3.65 \mathrm{E}-04$ & 8.00 & 0.000129 & 7.32 & $8.54 \mathrm{E}-05$ & 6.42 & $1.96 \mathrm{E}-04$ & 8.34 & $1.48 \mathrm{E}-04$ & 8.08 \\
\hline $1.65 \mathrm{E}-03$ & 12.74 & 4.04E-04 & 8.37 & 0.000144 & 7.58 & $9.64 \mathrm{E}-05$ & 6.61 & $2.35 \mathrm{E}-04$ & 8.77 & $1.87 \mathrm{E}-04$ & 8.47 \\
\hline 2.07E-03 & 13.26 & 7.50E-04 & 10.48 & 0.000188 & 7.99 & $1.02 \mathrm{E}-04$ & 6.92 & $2.74 \mathrm{E}-04$ & 9.13 & $2.05 \mathrm{E}-04$ & 8.88 \\
\hline & & $9.00 \mathrm{E}-04$ & 11.00 & 0.000401 & 9.70 & $1.15 \mathrm{E}-04$ & 7.14 & 3.33E-04 & 9.64 & $2.84 \mathrm{E}-04$ & 9.78 \\
\hline
\end{tabular}




\begin{tabular}{|c|c|c|c|c|c|c|c|c|c|}
\hline $4 \mathrm{~Pa}$ & & $1.8 \mathrm{~Pa}$ & & $0.5 \mathrm{~Pa}$ & & $0.2 \mathrm{~Pa}$ & & UHV & \\
\hline $\mathrm{da} / \mathrm{dN}$ (mm/cycle) & $\Delta \mathrm{K}(\mathrm{MPaVm})$ & $\mathrm{da} / \mathrm{dN}$ & $\Delta \mathrm{K}$ & $\mathrm{da} / \mathrm{dN}$ & $\Delta \mathrm{K}$ & $\mathrm{da} / \mathrm{dN}$ & $\Delta \mathrm{K}$ & $\mathrm{da} / \mathrm{dN}$ & $\Delta \mathrm{K}$ \\
\hline $7.69 \mathrm{E}-07$ & 1.96 & $1.04 \mathrm{E}-07$ & 1.71 & 2.77E-07 & 2.09 & $2.11 \mathrm{E}-07$ & 2.64 & $7.96 \mathrm{E}-07$ & 5.25 \\
\hline $1.01 \mathrm{E}-06$ & 2.10 & $1.87 \mathrm{E}-07$ & 1.81 & $5.34 \mathrm{E}-07$ & 2.27 & $3.00 \mathrm{E}-07$ & 2.71 & $1.28 \mathrm{E}-06$ & 5.52 \\
\hline $1.16 \mathrm{E}-06$ & 2.24 & $3.65 \mathrm{E}-07$ & 1.89 & 7.31E-07 & 2.45 & $4.89 \mathrm{E}-07$ & 2.83 & $2.30 \mathrm{E}-06$ & 5.80 \\
\hline $1.32 \mathrm{E}-06$ & 2.39 & $4.94 \mathrm{E}-07$ & 2.01 & $8.82 \mathrm{E}-07$ & 2.61 & $6.00 \mathrm{E}-07$ & 3.01 & 4.66E-06 & 5.97 \\
\hline $1.50 \mathrm{E}-06$ & 2.55 & $6.40 \mathrm{E}-07$ & 2.14 & $9.33 \mathrm{E}-07$ & 2.66 & 7.00E-07 & 3.17 & $6.60 \mathrm{E}-06$ & 6.08 \\
\hline $1.78 \mathrm{E}-06$ & 2.76 & 7.32E-07 & 2.30 & $1.00 \mathrm{E}-06$ & 2.90 & $9.50 \mathrm{E}-07$ & 3.39 & $9.02 \mathrm{E}-06$ & 6.31 \\
\hline $1.90 \mathrm{E}-06$ & 2.80 & $9.53 \mathrm{E}-07$ & 2.47 & $1.21 \mathrm{E}-06$ & 3.08 & $1.10 \mathrm{E}-06$ & 3.58 & $1.27 \mathrm{E}-05$ & 6.46 \\
\hline $2.00 \mathrm{E}-06$ & 2.89 & $1.22 \mathrm{E}-06$ & 2.61 & $1.30 \mathrm{E}-06$ & 3.29 & $1.30 \mathrm{E}-06$ & 3.78 & $1.75 \mathrm{E}-05$ & 6.81 \\
\hline 2.12E-06 & 3.08 & $1.36 \mathrm{E}-06$ & 2.78 & $1.50 \mathrm{E}-06$ & 3.50 & $1.50 \mathrm{E}-06$ & 3.87 & 2.23E-05 & 6.99 \\
\hline 2.57E-06 & 3.29 & $1.40 \mathrm{E}-06$ & 2.97 & $1.75 \mathrm{E}-06$ & 3.60 & $1.60 \mathrm{E}-06$ & 4.00 & $2.69 \mathrm{E}-05$ & 7.17 \\
\hline $2.79 \mathrm{E}-06$ & 3.50 & $1.55 \mathrm{E}-06$ & 3.10 & $1.80 \mathrm{E}-06$ & 3.85 & $2.00 \mathrm{E}-06$ & 4.21 & $2.82 \mathrm{E}-05$ & 7.40 \\
\hline $2.98 \mathrm{E}-06$ & 3.60 & $1.61 \mathrm{E}-06$ & 3.21 & $2.40 \mathrm{E}-06$ & 4.20 & $2.10 \mathrm{E}-06$ & 4.37 & 3.01E-05 & 7.45 \\
\hline $3.23 \mathrm{E}-06$ & 3.74 & $1.70 \mathrm{E}-06$ & 3.41 & $2.80 \mathrm{E}-06$ & 4.40 & $2.30 \mathrm{E}-06$ & 4.56 & $3.56 \mathrm{E}-05$ & 7.75 \\
\hline 4.01E-06 & 4.02 & $2.00 \mathrm{E}-06$ & 3.57 & 4.50E-06 & 4.80 & $2.75 \mathrm{E}-06$ & 4.80 & $4.01 \mathrm{E}-05$ & 8.01 \\
\hline $5.29 \mathrm{E}-06$ & 4.29 & $2.75 \mathrm{E}-06$ & 3.78 & 5.00E-06 & 4.90 & $3.50 \mathrm{E}-06$ & 5.05 & 4.32E-05 & 8.11 \\
\hline $6.63 \mathrm{E}-06$ & 4.45 & 3.50E-06 & 3.99 & $6.00 \mathrm{E}-06$ & 5.22 & 4.00E-06 & 5.17 & $5.63 \mathrm{E}-05$ & 8.50 \\
\hline $6.86 \mathrm{E}-06$ & 4.58 & 4.00E-06 & 4.35 & $9.00 \mathrm{E}-06$ & 5.47 & $5.60 \mathrm{E}-06$ & 5.33 & $6.79 \mathrm{E}-05$ & 8.75 \\
\hline $8.55 \mathrm{E}-06$ & 4.89 & 5.00E-06 & 4.56 & $1.33 \mathrm{E}-05$ & 5.93 & $7.21 \mathrm{E}-06$ & 5.40 & $8.40 \mathrm{E}-05$ & 9.25 \\
\hline $1.53 \mathrm{E}-05$ & 5.22 & $6.50 \mathrm{E}-06$ & 4.89 & $2.00 \mathrm{E}-05$ & 6.10 & $8.90 \mathrm{E}-06$ & 5.57 & $9.20 \mathrm{E}-05$ & 9.50 \\
\hline $2.85 \mathrm{E}-05$ & 5.57 & $8.50 \mathrm{E}-06$ & 5.07 & $2.75 \mathrm{E}-05$ & 6.42 & $1.29 \mathrm{E}-05$ & 5.95 & $1.11 \mathrm{E}-04$ & 10.00 \\
\hline $3.15 \mathrm{E}-05$ & 5.64 & $2.00 \mathrm{E}-05$ & 5.60 & 3.91E-05 & 6.95 & $2.27 \mathrm{E}-05$ & 6.31 & 1.19E-04 & 10.22 \\
\hline 4.73E-05 & 5.94 & $3.02 \mathrm{E}-05$ & 6.05 & $4.50 \mathrm{E}-05$ & 7.12 & $3.05 \mathrm{E}-05$ & 6.79 & $1.72 \mathrm{E}-04$ & 10.75 \\
\hline $6.01 \mathrm{E}-05$ & 6.34 & 5.05E-05 & 6.61 & 5.31E-05 & 7.53 & $3.61 \mathrm{E}-05$ & 7.08 & $1.91 \mathrm{E}-04$ & 11.08 \\
\hline $7.58 \mathrm{E}-05$ & 6.77 & $6.24 \mathrm{E}-05$ & 6.93 & $6.42 \mathrm{E}-05$ & 8.12 & $4.96 \mathrm{E}-05$ & 7.66 & $2.32 \mathrm{E}-04$ & 11.50 \\
\hline 8.59E-05 & 7.18 & $6.95 \mathrm{E}-05$ & 7.30 & $9.04 \mathrm{E}-05$ & 8.66 & $7.20 \mathrm{E}-05$ & 8.02 & $2.58 \mathrm{E}-04$ & 12.02 \\
\hline $9.66 \mathrm{E}-05$ & 7.22 & $9.00 \mathrm{E}-05$ & 7.78 & $1.00 \mathrm{E}-04$ & 9.00 & $8.11 \mathrm{E}-05$ & 8.48 & $3.45 \mathrm{E}-04$ & 13.03 \\
\hline 1.27E-04 & 7.71 & $1.04 \mathrm{E}-04$ & 8.32 & $1.29 \mathrm{E}-04$ & 9.43 & $9.33 \mathrm{E}-05$ & 8.91 & $3.53 \mathrm{E}-04$ & 13.03 \\
\hline $1.54 \mathrm{E}-04$ & 8.23 & 1.14E-04 & 8.57 & $2.30 \mathrm{E}-04$ & 10.61 & $1.11 \mathrm{E}-04$ & 8.92 & $5.02 \mathrm{E}-04$ & 13.75 \\
\hline $1.79 \mathrm{E}-04$ & 8.78 & $1.50 \mathrm{E}-04$ & 9.04 & $5.40 \mathrm{E}-04$ & 12.94 & 1.19E-04 & 9.22 & $6.52 \mathrm{E}-04$ & 14.50 \\
\hline $2.36 \mathrm{E}-04$ & 9.38 & $1.75 \mathrm{E}-04$ & 9.28 & 1.17E-03 & 15.39 & $1.24 \mathrm{E}-04$ & 9.35 & $8.71 \mathrm{E}-04$ & 15.28 \\
\hline
\end{tabular}

